\title{
Problems on permutation and irreducible polynomials over finite fields
}

\author{
by
}

Aleksandr Tuxanidy

A thesis submitted to the Faculty of Graduate and Postdoctoral Affairs in partial fulfillment of the requirements for the degree of

Doctor of Philosophy

in

Pure Mathematics

Carleton University

Ottawa, Ontario

(C) 2018

Aleksandr Tuxanidy 


\section{Contents}

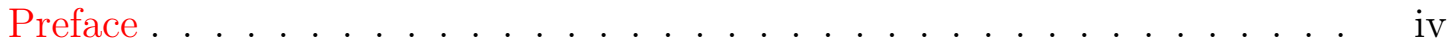

Abstract .....................

Acknowledgements . . . . . . . . . . . . . . . viii

1 Introduction 1

2 Preliminaries $\quad 29$

2.1 Inverses of linearized polynomials inducing bijections of subspaces . . 30

2.2 DFT for finite fields, period of functions and cyclotomic polynomials . 39

2.3 Gaussian sums and periods . . . . . . . . . . . . . . . 42

3 On the inverses of some classes of permutations of finite fields 48

3.1 Inverses of some classes of permutations $\ldots \ldots \ldots \ldots$

3.2 Compositional inverse of a general class . . . . . . . . . . . . 62

3.3 Explicit compositional inverse of a second class . . . . . . . . 71

4 Compositional inverses and complete mappings over finite fields $\quad \mathbf{8 0}$

4.1 Inverses of linearized binomials permuting kernels of traces . . . . . 82

4.1 .1 Statement and proof of result . . . . . . . . . . 82

4.1.2 Method used to obtain the inverse in Theorem 4.1.4 . . . . 87

4.2 Extensions of a class of complete permutation polynomials . . . . . 91 
4.3 Inverse of the complete mapping . . . . . . . . . . . . . . . 97

5 A new proof of the Hansen-Mullen irreducibility conjecture $\quad 102$

5.1 Least period of the DFT and connection to irreducible polynomials . 103

5.2 Characteristic elementary symmetric and delta functions . . . . . . 108

5.3 Least period of $\Delta_{w, c}$ and proof of Theorem 1.0.11 . . . . . . . 116

6 On the number of $N$-free elements with prescribed trace 122

6.1 A formula for $Z_{q, m, N} \ldots \ldots \ldots \ldots \ldots$

6.2 The case of the zero trace . . . . . . . . . . . . . . 127

6.2.1 Simplification of $Z_{q, m, N}(0)$ and direct consequences . . . . . . 128

6.2.2 Proof of Corollaries 1.0.23 and 1.0.25 . . . . . . . . . . 131

6.3 Uniformity in the case of the non-zero trace . . . . . . . . . . . 137

6.4 Connection to $P_{q, m, N}(c) \ldots \ldots \ldots \ldots \ldots$

$\begin{array}{llr}7 & \text { Conclusion } & 148\end{array}$

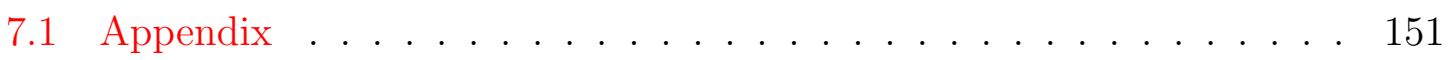

$\begin{array}{ll}\text { Bibliography } & 153\end{array}$ 


\section{Preface}

The content of this integrated thesis is primarily based on the following four published articles, co-authored jointly and under the supervision of Qiang Wang. These are released under a Creative Commons Attribution Non-Commercial No Derivatives License. In particular, all mathematical results of the thesis, such as theorems, corollaries, lemmas, propositions, examples, etc., were originally obtained in the following works. Thus in the case the reader wishes to refer to one of these results, he/she should cite instead the corresponding published article in the journal where it was published.

We list the works according to the order of the chapters in the thesis.

[70] A. Tuxanidy and Q. Wang, On the inverses of some classes of permutations of finite fields, Finite Fields and Their Applications, v.28 (2014), p.244-281. https: //doi.org/10.1016/j.ffa.2014.02.006

[71] A. Tuxanidy and Q. Wang, Compositional inverses and complete mappings over finite fields, Discrete Applied Mathematics, v.217 no.2 (2017), p.318-329. https : //doi.org/10.1016/j.dam.2016.09.009

[72] A. Tuxanidy and Q. Wang, A new proof of the Hansen-Mullen irreducibility conjecture, Canadian Journal of Mathematics, no.70 (2018), 1373-1389. https:// doi.org/10.4153/CJM-2017-022-1 
[69] A. Tuxanidy and Q. Wang, On the number of $N$-free elements with prescribed trace, Journal of Number Theory, v.160 (2016), p.536-565. https://doi.org/10. $1016 / j \cdot j n t .2015 .09 .008$

These articles are substantially reproduced in this thesis: The main content of these is reproduced in Chapters 3, 4, 5, 6, respectively. However some of the material in the works, notably parts of the introductions to each of the articles, as well as corresponding preliminaries sections, have been moved to the introduction here in Chapter 1, as well as to the Preliminaries, Chapter 2. This has been done in part to avoid repetitions of literary backgroud and basic concepts, as well as to present a more unified, self-contained work.

The author of the thesis has also jointly co-authored the following two other articles, which will nonetheless not make it into the thesis.

[73] A. Tuxanidy and Q. Wang, Characteristic digit-sum sequences, Cryptogr. Commun. (2018) 10:705-717

[68] A. Tuxanidy and Q. Wang, Composed products and factors of cyclotomic polynomials over finite fields, Designs, Codes and Cryptography (2013), v.69, no.2, p. $203-231$ 


\section{Abstract}

This integrated thesis is dedicated to the study of polynomials over finite fields, notably permutation polynomials and their compositional inverses, existence of irreducible polynomials with prescribed coefficients, as well as ennumeration of primitive polynomials with prescribed trace. Here we present several of the results, first attained in [69-72], by the author of the thesis in collaboration with Qiang Wang.

In Chapter 3 we showcase our study, in [70], of the compositional inverses of some general classes of permutation polynomials over finite fields. There we showed that we can write these inverses in terms of the inverses of two other polynomials bijecting subspaces of the finite field, where one of these is a linearized polynomial. In some cases we are able to explicitly obtain these inverses, thus obtaining the compositional inverse of the permutation in question. In addition we show how to compute a linearized polynomial inducing the inverse map over subspaces on which a prescribed linearized polynomial induces a bijection. We also obtain the explicit compositional inverses of two classes of permutation polynomials generalizing those whose compositional inverses were recently obtained.

In Chapter 4 we present our work from [71] where we study the compositional inverses of permutation polynomials and complete mappings over finite fields. Recently the compositional inverses of linearized permutation binomials were obtained in [83]. In Chapter 4 we commence by presenting the compositional inverses of a 
class of linearized binomials permuting the kernel of the trace map. Note that it was shown in our work in [70] that computing inverses of bijections of subspaces has applications in determining the compositional inverses of certain permutation classes related to linearized polynomials. Consequently, we give the compositional inverse of a new class of complete mappings. This complete mapping class extends several recent constructions given in $[46,63,85]$. We also construct recursively a class of complete mappings involving multi-trace functions.

In Chapter 5 we present our work in [72] where we give a new proof of the HansenMullen irreducibility conjecture. The proof relies on an application of a (seemingly new) sufficient condition for the existence of elements of degree $n$ in the support of functions on finite fields. This connection to irreducible polynomials is made via the least period of the discrete Fourier transform (DFT) of functions with values in finite fields. We exploit this relation and prove, in an elementary fashion, that a relevant function related to the DFT of characteristic elementary symmetric functions (which produce the coefficients of characteristic polynomials) has a sufficiently large least period (except for some genuine exceptions). This bears a sharp contrast to mainstream techniques in literature employed to tackle existence of irreducible polynomials with prescribed coefficients.

While in Chapter 5 we studied the existence of irreducible polynomials with prescribed coefficients, we are also interested in the ennumeration of irreducible polynomials, in particular primitive polynomials, with prescribed coefficients. Since there is a clear correspondence between primitive polynomials with prescribed trace and primitive elements with prescribed trace, it is equivalent to study the latter. In Chapter 6 we explore our work, in [69], regarding primitive elements, and more generally $N$-free elements, with prescribed trace. We derived a formula for the number of $N$ free elements over a finite field with prescribed trace, in particular trace zero, in terms 
of Gaussian periods. As a consequence, we obtain several explicit formulae in special cases. In addition we show that if all the prime factors of $q-1$ divide the degree of the extension, $m$, then the number of primitive elements in $\mathbb{F}_{q^{m}}$, with prescribed non-zero trace, is uniformly distributed. Finally we explore the related number of elements in $\mathbb{F}_{q^{m}}$ with multiplicative order $N$ and having a prescribed trace. 


\section{Acknowledgements}

First of all, I would like to thank my advisor, Qiang Wang, for all his help and tutelage throughout the years. I am very blessed to have met him, several years ago, and to have had the opportunity to work with him as a team. His enthusiasm for research and curiosity was always contagious from the start, and I always look up to him. I would also like to thank Emmanuel Lorin and Daniel Panario for all their support and guidance throughout the years.

The work in this thesis would not have been possible without the love, encouragement and support of family and friends, who have always been, and continue to be, there for me. Notably, it would not have been possible without my second, better, half. 


\section{Chapter 1}

\section{Introduction}

The topic of finite fields is classical in Mathematics. In recent decades, the advent of computer technology and communication systems has accelerated the resarch input of the mathematical community. In addition, these are fundamental objects in number theory, algebraic geometry, combinatorics, cryptography, coding theory, among others. Polynomials over finite fields are one of the main objects of research in the study of finite fields, both theoretically and practically.

This integrated thesis is devoted to the study of polynomials over finite fields, such as permutation polynomials and their compositional inverses, existence of irreducible polynomials satisfying constraints on their coefficients, and number of primitive polynomials (equivalently primitive elements; more generally, $N$-free elements) with prescribed trace ${ }^{1}$.

Let $q$ be a power of a prime number $p$ and let $\mathbb{F}_{q}$ be a finite field with $q$ elements. Let $\mathbb{F}_{q}[x]$ denote the ring of polynomials over $\mathbb{F}_{q}$. We call $f \in \mathbb{F}_{q}[x]$ a permutation polynomial $(\mathrm{PP})$ of $\mathbb{F}_{q}$ if it induces a permutation of $\mathbb{F}_{q}$. Note that since $x^{q}=x$ for all $x \in \mathbb{F}_{q}$, one only needs to consider polynomials of degree less than $q$. It is

\footnotetext{
${ }^{1}$ The content of this introduction is substantively based on the introductions on the four articles [69-72], written by the author of the thesis in collaboration with Qiang Wang. All rights reserved.
} 
clear that permutation polynomials form a group under composition and reduction modulo $x^{q}-x$ that is isomorphic to the symmetric group on $q$ letters. Thus for any permutation polynomial $f \in \mathbb{F}_{q}[x]$, there exists a unique $f^{-1} \in \mathbb{F}_{q}[x]$, of degree less than $q$, such that $f^{-1}(f(x)) \equiv f\left(f^{-1}(x)\right) \equiv x\left(\bmod x^{q}-x\right)$. Here $f^{-1}$ is defined as the compositional inverse of $f$, although we may simply call it sometimes the inverse of $f$ on $\mathbb{F}_{q}$. When we think of $x \in \mathbb{F}_{q}$ as fixed and view $f \in \mathbb{F}_{q}[x]$ as a mapping of $\mathbb{F}_{q}$, we call $f^{-1}(x)$ the preimage of $x$ under $f$.

The construction of permutation polynomials over finite fields is an old and difficult subject that continues to attract interest due to their applications in cryptography [61], [64], coding theory [25], [46], and combinatorics [26]. Recently much progress has taken place in this area. See for instance [3], [4], [5],[15], [24], [31], [38], [45], [50], [53], [78], [88], [91], [93], and the references therein for some previous works. However, how to go about inverting such permutations has remained unclear to the present. There is, in contrast, a marked absence of explicit formulas describing compositional inverses, but also a general lack of methods which may be useful to attain these formulas. Nevertheless there is some recent progress (see for instance [23, 76, 79, 81-83] to name a few).

In Chapter 3 we contribute to the growing literature on this topic and obtain the compositional inverses of several classes of PPs. The main result of this chapter could viewed as Theorem 1.0.2, obtained via a generalization of a novel method introduced by Wu-Liu in [81]. Theorem 1.0.2 gives a formula for the compositional inverse of a general class of PPs obtained by Akbary-Ghioca-Wang [3], and which may be found here in Theorem 1.0.1. We then apply Theorem 1.0.2 to obtain the compositional inverses of several classes of PPs from literature. See for instance Theorems 1.0.10, 3.1.10, 3.2.4 and Corollaries 1.0.4, 1.0.6, 3.1.2, 3.1.3, 3.1.4, 3.1.11 to name a few.

We say that a polynomial $L(x) \in \mathbb{F}_{q^{n}}[x]$ is linearized, also called a q-polynomial, if 
$L(x)$ has the shape $L(x)=\sum_{i} a_{i} x^{q^{i}}$ for some coefficients $a_{i} \in \mathbb{F}_{q^{n}}$. An important class of such polynomials is the trace function $T_{q^{n} \mid q}(x)=\sum_{i=0}^{n-1} x^{q^{i}}$. When it will not cause confusion, we write $T$ instead of $T_{q^{n} \mid q}$. In [81], Wu-Liu introduced a method to obtain the compositional inverse of the class $f(x)=x\left(L\left(T_{q^{n} \mid q}(x)\right)+a T_{q^{n} \mid q}(x)+a x\right)$ of PPs of $\mathbb{F}_{q^{n}}$, in the case when $q$ is even, $n$ is odd, $L \in \mathbb{F}_{q}[x]$ is a 2-polynomial and $a \in \mathbb{F}_{q}^{*}$. WuLiu start with a decomposition of the finite field, $\mathbb{F}_{q^{n}}$, into the direct sum $\mathbb{F}_{q} \oplus \operatorname{ker}(T)$. This has the effect of converting the problem of computing the inverse of $f$ into the problem of computing the inverse of a bivariate function permuting $\mathbb{F}_{q} \oplus \operatorname{ker}(T)$. This in turn is equivalent to obtaining the inverses of two permutation polynomials, $\bar{f}, \varphi$, over the subspaces $\mathbb{F}_{q}$ and $\operatorname{ker}(T)$, respectively, where $\varphi$ is a 2-polynomial (in fact, quadratic). As $q$ is even and $n$ is odd, $T$ is idempotent, i.e., $T \circ T=T$, and thus the (bijective) transformation $\phi: \mathbb{F}_{q^{n}} \rightarrow \mathbb{F}_{q} \oplus \operatorname{ker}(T)$ can be defined by $\phi(x)=(T(x), x-T(x))$, since $T\left(\mathbb{F}_{q^{n}}\right)=\mathbb{F}_{q}$ additionally. Because computing the inverse of any $p$-polynomial permutation on a subspace of $\mathbb{F}_{q^{n}}$ amounts to solving a system of linear equations, this problem is thus finally reduced to computing the inverse of $\left.\bar{f}\right|_{\mathbb{F}_{q}}$.

In Chapter 3 we extend this useful method to other more general classes of permutation polynomials given in [3], and [88], particularly written in terms of arbitrary linearized polynomials $\psi($ instead of just $T$ ). We write their inverses in terms of the inverses of two other polynomials, one of these being linearized, over subspaces. In some special cases we can determine these inverses, thus obtaining the full result. However, given that we may not always have a "nice enough" expression for $\operatorname{ker}(\psi)$, we instead use the map $\phi_{\psi}: \mathbb{F}_{q^{n}} \rightarrow \psi\left(\mathbb{F}_{q^{n}}\right) \oplus S_{\psi}$, where $S_{\psi}:=\left\{x-\psi(x) \mid x \in \mathbb{F}_{q^{n}}\right\}$ - a subspace of $\mathbb{F}_{q^{n}}$, in similarity with $\operatorname{ker}(T)$ as above - defined by $\phi_{\psi}(x)=(\psi(x), x-\psi(x))$. In the case that $\psi$ induces an idempotent map of $\mathbb{F}_{q^{n}}, S_{\psi}=\operatorname{ker}(\psi)$. As $\phi_{\psi}$ is injective 
for any such $\psi$ (but not surjective in the case that $\psi$ is not idempotent), we similarly transform the problem of computing the inverses of permutation polynomials into the problem of computing the inverses of two other bijections, $\bar{f}, \varphi$, over the subspaces $\psi\left(\mathbb{F}_{q^{n}}\right)$ and $S_{\psi}$, respectively.

Many of our resulting expressions for compositional inverses, or preimages in some cases, are particularly written in terms of linearized polynomials inducing the inverse map over subspaces on which prescribed linearized polynomials induce a bijection. Nonetheless, in Theorem 2.1.5 we show how to obtain such compositional inverses on subspaces. As we show there, obtaining the coefficients of such linearized polynomials is equivalent to solving a system of linear equations. However in order to set up such a system one is required to first obtain a linearized polynomial, $K \in \mathscr{L}_{n}\left(\mathbb{F}_{q^{n}}\right)$, inducing an idempotent map with a prescribed kernel (we can always do this). But clearly, if we are given an idempotent $\psi \in \mathscr{L}_{n}\left(\mathbb{F}_{q^{n}}\right)$, i.e., $S_{\psi}=\operatorname{ker}(\psi)$, and $S_{\psi}$ is such a prescribed kernel, then one can simply let $K=\psi$. In the simultaneous case that the characteristic $p$ does not divide $n$, the coefficients of $\varphi \in \mathscr{L}_{n}\left(\mathbb{F}_{q^{n}}\right)$ belong to $\mathbb{F}_{q}$ (i.e., the associate Dickson matrix is circulant), and $\varphi$ induces a bijection between two $\mathbb{F}_{q}$-subspaces, one can quickly solve the corresponding linear system by using the Circular Convolution Theorem, for the Discrete Fourier Transform (DFT), together with a Fast Fourier Transform (FFT). See [87] for details regarding circulants and their close relation to the DFT.

Before we delve any further, let us fix some notations. If we view $f \in \mathbb{F}_{q}[x]$ as a map of $\mathbb{F}_{q}$ and are given a subspace $V$ of $\mathbb{F}_{q}$, we denote by $\left.f\right|_{V}$ the map obtained by restricting $f$ to $V$. If $f$ is a bijection from $V$ to a subspace $W$ of $\mathbb{F}_{q}$, we mean by $\left.f^{-1}\right|_{W}$ the inverse map of $\left.f\right|_{V}$. When the context is clear we may however mean by $\left.f^{-1}\right|_{W}$ a polynomial in $\mathbb{F}_{q}[x]$ inducing the inverse map of $\left.f\right|_{V}$. In this case we call $\left.f^{-1}\right|_{W}$ the compositional inverse of $f$ over $V$. In both of the following cases, if $f(x)$ 
is viewed as an element of $\mathbb{F}_{q}$, or a polynomial in $\mathbb{F}_{q}[x]$, we denote $1 / f(x):=f(x)^{q-2}$. Thus for instance $1 / 0=0$ with our notation.

As an example of our results, we obtain in Theorem 1.0.2 the compositional inverse of the permutation $f$ in the following theorem, under the assumption that $\varphi$ bijects $S_{\psi}$, and written in terms of the inverses of $\left.\bar{f}\right|_{\psi\left(\mathbb{F}_{q^{n}}\right)}$ and $\left.\varphi\right|_{S_{\psi}}$. Recall that for a linearized polynomial $\psi$, we defined $S_{\psi}$ to be the subspace $\left\{\alpha-\psi(\alpha): \alpha \in \mathbb{F}_{q^{n}}\right\}$.

Theorem 1.0.1 (Theorem 5.1, [3]). Consider any polynomial $g \in \mathbb{F}_{q^{n}}[x]$, any additive polynomials $\varphi, \psi \in \mathbb{F}_{q^{n}}[x]$, any q-polynomial $\bar{\psi} \in \mathbb{F}_{q^{n}}[x]$ satisfying $\varphi \circ \psi=$ $\bar{\psi} \circ \varphi$ and $\left|\psi\left(\mathbb{F}_{q^{n}}\right)\right|=\left|\bar{\psi}\left(\mathbb{F}_{q^{n}}\right)\right|$, and any polynomial $h \in \mathbb{F}_{q^{n}}[x]$ such that $h\left(\psi\left(\mathbb{F}_{q^{n}}\right)\right) \subseteq$ $\mathbb{F}_{q} \backslash\{0\}$. Then

$$
f(x)=h(\psi(x)) \varphi(x)+g(\psi(x))
$$

permutes $\mathbb{F}_{q^{n}}$ if and only if

(i) $\operatorname{ker}(\varphi) \cap \operatorname{ker}(\psi)=\{0\}$; and

(ii) $\bar{f}(x):=h(x) \varphi(x)+\bar{\psi}(g(x))$ is a bijection from $\psi\left(\mathbb{F}_{q^{n}}\right)$ to $\bar{\psi}\left(\mathbb{F}_{q^{n}}\right)$.

Theorem 1.0.2. Using the same notations and assumptions of Theorem 1.0.1, assume that $f$ is a permutation of $\mathbb{F}_{q^{n}}$, and further assume that $\left|S_{\psi}\right|=\left|S_{\bar{\psi}}\right|$ and $\operatorname{ker}(\varphi) \cap \psi\left(S_{\psi}\right)=\{0\}$. Then $\varphi$ induces a bijection from $S_{\psi}$ to $S_{\bar{\psi}}$. Let $\bar{f}^{-1},\left.\varphi^{-1}\right|_{S_{\bar{\psi}}} \in$ $\mathbb{F}_{q^{n}}[x]$ induce the inverses of $\left.\bar{f}\right|_{\psi\left(\mathbb{F}_{q^{n}}\right)}$ and $\left.\varphi\right|_{S_{\psi}}$, respectively. Then the compositional inverse of $f$ on $\mathbb{F}_{q^{n}}$ is given by

$$
f^{-1}(x)=\bar{f}^{-1}(\bar{\psi}(x))+\left.\varphi^{-1}\right|_{S_{\bar{\psi}}}\left(\frac{x-\bar{\psi}(x)-g\left(\bar{f}^{-1}(\bar{\psi}(x))\right)+\bar{\psi}\left(g\left(\bar{f}^{-1}(\bar{\psi}(x))\right)\right)}{h\left(\bar{f}^{-1}(\bar{\psi}(x))\right)}\right) .
$$

Furthermore, if $\varphi$ induces a bijection from $\psi\left(\mathbb{F}_{q^{n}}\right)$ to $\bar{\psi}\left(\mathbb{F}_{q^{n}}\right)$, then $\varphi$ is a permutation 
of $\mathbb{F}_{q^{n}}$ and the compositional inverse of $f$ on $\mathbb{F}_{q^{n}}$ is given by

$$
f^{-1}(x)=\varphi^{-1}\left(\frac{x-g\left(\bar{f}^{-1}(\bar{\psi}(x))\right)}{h\left(\bar{f}^{-1}(\bar{\psi}(x))\right)}\right)
$$

Note that condition (i) and the fact that the images of $\psi, \bar{\psi}$, are equally sized in Theorem 1.0.1 imply that $\varphi$ is a bijection from $\operatorname{ker}(\psi)$ to $\operatorname{ker}(\bar{\psi})$, for instance not necessarily satisfying $\operatorname{ker}(\varphi) \cap S_{\psi}=\{0\}$ (even though $\operatorname{ker}(\psi) \subseteq S_{\psi}$ ) required to attain injectivity on $S_{\psi}$, which is an imposed hypothesis of Theorem 1.0.2. Thus our result is further restricted by our imposed assumption that $\varphi$ bijects $S_{\psi}$, a superset of $\operatorname{ker}(\psi)$. However, as previously described in the Preliminaries chapter, in the case that $\psi$ is idempotent or $\varphi$ is a permutation of $\mathbb{F}_{q^{n}}$, we can get the inverse of $\varphi$ on $S_{\psi}$, and hence obtain the compositional inverse of $f$ in terms of a polynomial inducing the inverse map of $\left.\bar{f}\right|_{\psi\left(\mathbb{F}_{q^{n}}\right)}$. Note that one may find several linearized polynomials inducing idempotent endomorphisms of $\mathbb{F}_{q^{n}}$. See Remark 2.1.9 for more details regarding this.

As a consequence of this result we obtain several corollaries, like the following, in terms of the inverse of $\left.\bar{f}\right|_{\psi\left(\mathbb{F}_{q^{n}}\right)}$ and the inverse of a linearized permutation polynomial of $\mathbb{F}_{q^{n}}$, which is already known (see Proposition 2.1.1).

Remark 1.0.3. To clarify an ambiguity in the presentation of our results: In many of the following results we make citations in the style of "See Theorem ' $x$ '..." or "See Corollary ' $x$ '..." to refer to a result in another paper where the construction of the considered permutation polynomial was obtained. However the compositional inverses given here in the statements of these results are ours.

Corollary 1.0.4 (See Theorem 5.1 (c), [3]). Consider q-polynomials $\varphi, \psi, \bar{\psi} \in \mathbb{F}_{q^{n}}[x]$ satisfying $\varphi \circ \psi=\bar{\psi} \circ \varphi$ and $\left|\psi\left(\mathbb{F}_{q^{n}}\right)\right|=\left|\bar{\psi}\left(\mathbb{F}_{q^{n}}\right)\right|$. Let $g, h \in \mathbb{F}_{q^{n}}[x]$ be such that 
$\left.(\bar{\psi} \circ g)\right|_{\psi\left(\mathbb{F}_{q^{n}}\right)}=0$ and $h\left(\psi\left(\mathbb{F}_{q^{n}}\right)\right) \subseteq \mathbb{F}_{q} \backslash\{0\}$. If

$$
f(x)=h(\psi(x)) \varphi(x)+g(\psi(x))
$$

permutes $\mathbb{F}_{q^{n}}$, then $\varphi$ permutes $\mathbb{F}_{q^{n}}$ as well, and the inverse of $f$ on $\mathbb{F}_{q^{n}}$ is given by

$$
f^{-1}(x)=\frac{\varphi^{-1}\left(x-g\left(\frac{\varphi^{-1}(\bar{\psi}(x))}{h\left(\bar{f}^{-1}(\bar{\psi}(x))\right)}\right)\right)}{h\left(\bar{f}^{-1}(\bar{\psi}(x))\right)}
$$

where $\bar{f}(x):=h(x) \varphi(x)$ induces a bijection from $\psi\left(\mathbb{F}_{q^{n}}\right)$ to $\bar{\psi}\left(\mathbb{F}_{q^{n}}\right)$. In particular, if $h\left(\psi\left(\mathbb{F}_{q^{n}}\right)\right)=\{c\}$ for some $c \in \mathbb{F}_{q} \backslash\{0\}$, then $f$ permutes $\mathbb{F}_{q^{n}}$ if and only if $\varphi$ permutes $\mathbb{F}_{q^{n}}$, in which case the compositional inverse of $f$ over $\mathbb{F}_{q^{n}}$ is given by

$$
f^{-1}(x)=c^{-1} \varphi^{-1}\left(x-g\left(c^{-1} \varphi^{-1}(\bar{\psi}(x))\right)\right) .
$$

Note in the above that, in particular, when $h(x)=c \in \mathbb{F}_{q} \backslash\{0\}$, the inverse of $f$ is given in terms of the inverse of $\varphi$ on $\mathbb{F}_{q^{n}}$, which may be obtained via an application of Proposition 2.1.1. As a result of Corollary 1.0.4 we also obtain Corollaries 3.1.2, 3.1.3, and 3.1.4. Here is one example of Corollary 1.0.4 using the fact that $T(x)^{q}-T(x)=0$.

Proposition 1.0.5. Let $G \in \mathbb{F}_{q^{2}}[x]$ be arbitrary, let $Q(x):=x^{q}-x$, let $g=T \circ G$, let $h(x)=c \in \mathbb{F}_{q} \backslash\{0\}$, and let $\varphi(x)=a x^{q}+b x$, where $a, b \in \mathbb{F}_{q}$ are such that $a \neq \pm b$. Then both $\varphi$ and

$$
f(x)=h(Q(x)) \varphi(x)+g(Q(x))=c\left(a x^{q}+b x\right)+T \circ G \circ Q(x)
$$


are permutations of $\mathbb{F}_{q^{2}}$, and the compositional inverse of $f$ on $\mathbb{F}_{q^{2}}$ is given by

$$
f^{-1}(x)=\frac{a x^{q}-b x}{c\left(a^{2}-b^{2}\right)}-\frac{T \circ G \circ\left(\frac{Q(x)}{c(b-a)}\right)}{c(a+b)} .
$$

Another consequence of Theorem 1.0.2 is the following.

Corollary 1.0.6 (See Theorem 1, [50]). Let $\varphi \in \mathbb{F}_{q}[x]$ be a q-polynomial permuting $\mathbb{F}_{q^{n}}$, let $G \in \mathbb{F}_{q}[x]$, let $\gamma \in \mathbb{F}_{q^{n}}$, let $c=T(\gamma)$, and assume that

$$
f(x)=\varphi(x)+\gamma G(T(x))
$$

permutes $\mathbb{F}_{q^{n}}$. Then the inverse of $f$ on $\mathbb{F}_{q^{n}}$ is given by

$$
f^{-1}(x)=\varphi^{-1}\left(x-\gamma G\left(\bar{f}^{-1}(T(x))\right)\right)
$$

where $\bar{f}(x):=c G(x)+\varphi(1) x$ is a permutation of $\mathbb{F}_{q}$.

Note in the case that $G$ is a $q$-polynomial, $\left.\bar{f}\right|_{\mathbb{F}_{q}}(x)=(c G(1)+\varphi(1)) x$; thus $\bar{f}^{-1}(T(x))=T(x) /(c G(1)+\varphi(1))$ and hence we can obtain the inverse of the permutation polynomial, $f$, by computing only $\varphi^{-1}$. We thus obtain Corollary 3.1.5. We also note that if we take $G(x)=x^{r}$ and $c=\varphi(1)$, then $\bar{f}(x)$ in Corollary 1.0.6 has the form $c\left(x^{r}+x\right)$ whose inverse can be explicitly computed (see [54], [76], [77]). Therefore the inverse of $f$ is again only dependent on $\varphi^{-1}$ which can also be obtained in terms of cofactors of the associate Dickson matrix by Proposition 2.1.1. Another straightforward application of Corollary 1.0.6 is the following example.

Proposition 1.0.7. As before, let $\varphi(x)=a x^{q}+b x$ where $a, b \in \mathbb{F}_{q}$ are such that $a \neq \pm b$, let $G(x)=x$, let $c=T(\gamma)$, where $\gamma \in \mathbb{F}_{q^{2}}$ is such that $a+b+c \neq 0$. Then 
both $\varphi$ and

$$
f(x)=\varphi(x)+\gamma T(x)
$$

are permutations of $\mathbb{F}_{q^{2}}$, and the compositional inverse of $f$ on $\mathbb{F}_{q^{2}}$ is given by

$$
f^{-1}(x)=\frac{a x^{q}-b x}{a^{2}-b^{2}}-\frac{\left(a \gamma^{q}-b \gamma\right) T(x)}{\left(a^{2}-b^{2}\right)(a+b+c)}
$$

We have also obtained the following compositional inverse, given in terms of the inverse of a linearized permutation polynomial, which can be obtained.

Corollary 1.0.8 (See Corollary 6.2, [88]). Let $n$ and $k$ be positive integers such that $\operatorname{gcd}(n, k)=d>1$, let $s$ be any positive integer with $s\left(q^{k}-1\right) \equiv 0\left(\bmod q^{n}-1\right)$. Let

$$
L_{1}(x)=\sum_{i=0}^{n / d-1} a_{i} x^{q^{i d}}, a_{i} \in \mathbb{F}_{q}
$$

be a $q^{d}$-polynomial with $L_{1}(1)=0$, let $L_{2}$ be a q-polynomial over $\mathbb{F}_{q}$, and let $G \in$ $\mathbb{F}_{q^{n}}[x]$. Assume

$$
f(x)=\left(G\left(L_{1}(x)\right)\right)^{s}+L_{2}(x)
$$

permutes $\mathbb{F}_{q^{n}}$. Then $L_{2}$ is a permutation of $\mathbb{F}_{q^{n}}$, and the inverse of $f$ on $\mathbb{F}_{q^{n}}$ is given by

$$
f^{-1}(x)=L_{2}^{-1}\left(x-\left(G \circ L_{2}^{-1} \circ L_{1}(x)\right)^{s}\right) .
$$

As a result of Corollary 1.0.8 we obtain Corollary 3.1.8 as well. One more example, in this case independent of Theorem 1.0.2, is the following. It makes use of a slight modification of the permutation construction given in Theorem 6.1, [3] (also appearing as Corollary 3.2 in [88]), which now takes into account the fact that $p$-polynomials over $\mathbb{F}_{q}$ commute with $q$-polynomials over $\mathbb{F}_{p}$. Let us first define the natural map 
$v_{q, n}: \mathscr{L}_{n}\left(\mathbb{F}_{q^{n}}\right) \rightarrow \mathbb{F}_{q^{n}}^{n}$ given by $v_{q, n}\left(\sum_{i=0}^{n-1} a_{i} x^{q^{i}}\right)=\left(a_{0} a_{1} \cdots a_{n-1}\right)$ (for simplicity we write vectors horizontally).

Theorem 1.0.9 (See Theorem 6.1, [3]). Let $q=p^{m}$ be the power of a prime number $p$, let $L_{1}, L_{2}$ be p-polynomials over $\mathbb{F}_{q}$, let $L_{3}$ be a q-polynomial over $\mathbb{F}_{p}$, and let $w \in \mathbb{F}_{q^{n}}[x]$ such that $w\left(L_{3}\left(\mathbb{F}_{q^{n}}\right)\right) \subseteq \mathbb{F}_{q}$. Then

$$
f(x)=L_{1}(x)+L_{2}(x) w\left(L_{3}(x)\right)
$$

permutes $\mathbb{F}_{q^{n}}$ if and only if $\bar{f}(x):=L_{1}(x)+L_{2}(x) w(x)$ is a permutation of $L_{3}\left(\mathbb{F}_{q^{n}}\right)$, and $\varphi_{y}(x):=L_{1}(x)+L_{2}(x) w(y)$ is a permutation of $\operatorname{ker}\left(L_{3}\right)$ for any $y \in L_{3}\left(\mathbb{F}_{q^{n}}\right)$.

If $f$ induces a permutation of $\mathbb{F}_{q^{n}}$, we have:

(a) If $x \in \mathbb{F}_{q^{n}}$ is such that $\operatorname{ker}\left(\varphi_{\bar{f}^{-1}\left(L_{3}(x)\right)}\right) \cap L_{3}\left(S_{L_{3}}\right)=\{0\}$, then the preimage of $x$ under $f$ is given by

$$
f^{-1}(x)=\bar{f}^{-1}\left(L_{3}(x)\right)+\left.\varphi_{\bar{f}-1\left(L_{3}(x)\right)}^{-1}\right|_{S_{L_{3}}}\left(x-L_{3}(x)\right) .
$$

(b) If $L_{3}$ is idempotent, then the compositional inverse of $f$ on $\mathbb{F}_{q^{n}}$ is given by

$$
f^{-1}(x)=\bar{f}^{-1}\left(L_{3}(x)\right)+\varphi_{\bar{f}^{-1}\left(L_{3}(x)\right)}^{-1} \mid \operatorname{ker}\left(L_{3}\right)\left(x-L_{3}(x)\right)
$$

where, for any fixed $y \in L_{3}\left(\mathbb{F}_{q^{n}}\right)$, the coefficients, $v_{p, m n}\left(\left.\varphi_{y}^{-1}\right|_{\operatorname{ker}\left(L_{3}\right)}\right)$, of a p-polynomial $\left.\varphi_{y}^{-1}\right|_{\operatorname{ker}\left(L_{3}\right)}$ inducing the inverse map of $\left.\varphi_{y}\right|_{\operatorname{ker}\left(L_{3}\right)}$, are a solution to the linear equation

$$
v_{p, m n}\left(\left.\varphi_{y}^{-1}\right|_{\operatorname{ker}\left(L_{3}\right)}\right) D_{\varphi_{y}}=v_{p, m n}\left(x-L_{3}(x)\right)
$$

where $D_{\varphi_{y}}$ is the $m n \times m n$ Dickson matrix associated with the p-polynomial $\varphi_{y}$.

(c) If $x \in \mathbb{F}_{q^{n}}$ is such that $\varphi_{\bar{f}^{-1}\left(L_{3}(x)\right)}$ permutes $L_{3}\left(\mathbb{F}_{q^{n}}\right)$, then $\varphi_{\bar{f}^{-1}\left(L_{3}(x)\right)}$ permutes 
$\mathbb{F}_{q^{n}}$, and the preimage of $x$ under $f$ is given by

$$
f^{-1}(x)=\varphi_{\bar{f}^{-1}\left(L_{3}(x)\right)}^{-1}(x) .
$$

We remark that Theorem 1.0.9 (b), (c), is a generalization, albeit non-explicit, of the PPs and their inverses considered in [23], [81].

Finally in Section 3.3 we prove Theorem 1.0.10 below, which gives the explicit compositional inverse of a class of permutation polynomials generalizing that of a linearized permutation class whose inverse was recently obtained in [79]. See also Lemma 3.3.3 which corresponds to the case when $G(x)=x$ and $c=1$ below. In particular, the method employed here to obtain such a result, as an application of Theorem 1.0.2, seems considerably less complicated than that of [79].

Theorem 1.0.10. Let $\alpha \in \mathbb{F}_{q^{n}}, c \in \mathbb{F}_{q}^{*}$ and $G \in \mathbb{F}_{q^{n}}[x]$ be arbitrary. Then

$$
F(x)=c\left(x^{q}-x+T(\alpha x)\right)+G(T(\alpha x))^{q}-G(T(\alpha x))
$$

is a permutation polynomial over $\mathbb{F}_{q^{n}}$ if and only if $T(\alpha) \neq 0$ and the characteristic, $p$, of $\mathbb{F}_{q}$, does not divide $n$. In this case the compositional inverse of $F$ is

$F^{-1}(x)=c^{-1}\left[T(\alpha)^{-1} n^{-1}(T(x)+B(x))+T(\alpha)^{-1} T\left(\alpha G\left(c^{-1} n^{-1} T(x)\right)\right)-G\left(c^{-1} n^{-1} T(x)\right)\right]$,

where the coefficients of $B(x)=\sum_{k=0}^{n-1} b_{k} x^{k^{k}} \in \mathbb{F}_{q^{n}}[x]$ are given by

$$
b_{k}=\sum_{j=1}^{n-1} j \alpha^{q^{j}}-n \sum_{l=k+1}^{n-1} \alpha^{q^{l}}, \quad 0 \leq k \leq n-1 .
$$

An important class of permutation polynomials are the so called complete permuta- 
tion polynomials $(\mathrm{CPP})$ over $\mathbb{F}_{q}$, also called complete mappings. These, by definition, are permutation polynomials $f \in \mathbb{F}_{q}[x]$ such that $f(x)+x$ is also a permutation polynomial over $\mathbb{F}_{q}$. CPPs have recently become a strong source of interest due to their connection to combinatorial objects such as orthogonal Latin squares [29,62, 63], and due to their applications in cryptography; in particular, in the construction of bent functions $[55,56,63,66]$. See also $[67,84-86]$ and the references therein for some recent work in the area. In Chapter 4 we study complete mappings and give several results in this area. Theorem 4.2.3 there represents an improvement to a result of Wu-Lin [84]. This result generalized some earlier corresponding ones found in $[47,63,85,86]$. We also give a recursive construction of complete mappings involving multi-trace functions (see Theorem 4.2.6). In addition we employ the CPP class of Theorem 4.2.3 to construct a set of mutually orthogonal complete mappings (see Corollary 4.2.8). Two mappings $f, g$, of $\mathbb{F}_{q}$, are said to be orthogonal if $f-g$ permutes $\mathbb{F}_{q}$. Note in particular that from such a set one may also readily obtain a set of mutually orthogonal Latin squares, an object of special interest in the literature (see for example [29]). As an application of Theorem 4.1.4 where we obtained the compositional inverses of linearized binomials permuting the kernel of the trace, we derive in Chapter 4 the complete permutation class in Theorem 4.2.3 generalizing some of the classes recently studied in $[47,63,84-86]$. Note that since inverses of complete mappings are also complete mappings, Theorem 4.3.2 and Corollary 4.3.3 imply the construction of a new, if rather complicated, class of complete permutation polynomials.

Another fundamental object of study in finite fields are irreducible polynomials. These are polynomials $f(x) \in \mathbb{F}_{q}[x]$ which can not be factored into a product of polynomials over $\mathbb{F}_{q}$ of degree less than that of $f$. An important class of irreducible polynomials are the so called primitive polynomials. These are the irreducibles whose roots are generators of the multiplicative group of their splitting field. Such generators 
are called primitive elements.

In 1992, Hansen-Mullen [37] conjectured (in Conjecture B there; see Theorem 1.0.11 below) that, except for a few genuine exceptions, there exist irreducible (and more strongly primitive; see Conjecture A in [37]) polynomials of degree $n$ over $\mathbb{F}_{q}$ with any one of its coefficients prescribed to any value. Theorem 1.0.11 was first proved by Wan [75] in 1997 for $q>19$ or $n \geq 36$, with the remaining cases being computationally verified soon after by Ham-Mullen [35]. First for a polynomial $f \in \mathbb{F}_{q}[x]$ and an integer $w$, we let $\left[x^{w}\right] f(x)$ denote the coefficient of $x^{w}$ in $f(x)$.

Theorem 1.0.11. Let $q$ be a power of a prime, let $c \in \mathbb{F}_{q}$, and let $n \geq 2$ and $w$ be integers with $1 \leq w \leq n$. If $w=n$, assume that $c \neq 0$. If $(n, w, c)=(2,1,0)$, further assume $q$ is odd. Then there exists a monic irreducible polynomial $P(x)$ of degree $n$ over $\mathbb{F}_{q}$ with $\left[x^{n-w}\right] P(x)=c$.

In 2006, Cohen [19], particularly building on some of the work of Fan-Han [30] on $p$-adic series, proved there exists a primitive polynomial of degree $n \geq 9$ over $\mathbb{F}_{q}$ with any one of its coefficients prescribed. The remaining cases of Conjecture A were settled by Cohen-Prešern in [21,22]. Cohen [19] and Cohen-Prešern [21,22] also gave theoretical explanations for the small cases of $q, n$, missed out in Wan's original proof [75].

The Hansen-Mullen conjectures have since been generalized to encompass results on the existence of irreducible and particularly primitive polynomials with several prescribed coefficients (see for instance $[33,34,57,58,74]$ for general irreducibles and $[18,36,59,65]$ for primitives). In particular Ha [34], building on some of the work of Pollack [58] and Bourgain [8], has recently proved that, for large enough $q, n$, there exists irreducibles of degree $n$ over $\mathbb{F}_{q}$ with roughly any $n / 4$ coefficients prescribed to any value. This seems to be the current record on the number of arbitrary coefficients one may prescribe to any values in an irreducible polynomial of degree $n$. 
There are some differences of approach in tackling existence questions of either general irreducible or primitive polynomials with prescribed coefficients. For instance, when working on irreducibles, and following in the footsteps of Wan [75], it has been common practice to exploit the $\mathbb{F}_{q}[x]$-analogue of Dirichlet's theorem for primes in arithmetic progressions; all this is done via Dirichlet characters on $\mathbb{F}_{q}[x], L$-series, zeta functions, etc. See for example [74]. Recently Pollack [58] and Ha [34], building on some ideas of Bourgain [8], applied the circle method to prove the existence of irreducible polynomials with several prescribed coefficients. On the other hand, in the case of primitives, the problem is usually approached via $p$-adic rings or fields (to account for the inconvenience that Newton's identities "break down", in some sense, in fields of positive characteristic) together with Cohen's sieving lemma, Vinogradov's characteristic function, etc. (see for example $[19,30]$ ). However there is one common feature these methods share, namely, when bounding the "error" terms comprised of character sums, the function field analogue of Riemann's hypothesis (Weil's bound) is used (perhaps without exception here). Nevertheless as a consequence of its $O\left(q^{n / 2}\right)$ nature it transpires a difficulty in extending the $n / 2$ threshold for the number of coefficients one can prescribe in irreducible or particularly primitive polynomials of degree $n$.

As the reader can take from all this, there seems to be a preponderance of the analytic method to tackle the existence problem of irreducibles and primitives with several prescribed coefficients. One then naturally wonders whether other view-points may be useful for tackling such problems. As Panario points out in [40], p.115,

The long-term goal here is to provide existence and counting results for irreducibles with any number of prescribed coefficients to any given values. This goal is completely out of reach at this time. Incremental steps seem doable, but it would be most interesting if new techniques were introduced 
to attack these problems [40]

In Chapter 5 we take a different approach and give a new proof of the HansenMullen irreducibility conjecture (or theorem), stated in Theorem 1.0.11. We attack the problem by studying the least period of certain functions related to the discrete Fourier transform (DFT) of characteristic elementary symmetric functions (which produce the coefficients of characteristic polynomials).

We emphasize that the work constitutes a sharp contrast to the more mainstream techniques in literature. The heart of the proof relies on Lemma 1.0.13 below, which gives a sufficient condition for a given polynomial to have an irreducible factor of degree $n$. This together with the exploitation of $q$-symmetric and $r$-periodic structures, as done in Dorsey-Hales [28], yields the result. Our proof theoretically explains, in a unified way, every case of the Hansen-Mullen conjecture. These include the small cases missed out in Wan's original proof [75], computationally verified by Ham-Mullen in [35]. However we should point out that, in contrast, our proof has the disadvantage of not yielding estimates for the number of irreducibles with a prescribed coefficient. It merely asserts their existence. We wonder whether some of the techniques introduced here can be extended to tackle the existence question for several prescribed coefficients, but we for now leave this to the consideration of the interested reader.

The proof relies in an application of the sufficient condition in Lemma 1.0.13 below, which follows from that in (i) of the following lemma. First for a function $F$ on a set $A$, let $\operatorname{supp}(F):=\{a \in A: F(a) \neq 0\}$ be the support of $F$. It should be observed that, in practice, $F$ will be defined on (a subset of the field) $\mathbb{F}_{q^{n}}$ or on a set of integers. For example, both usages occur in the proof of Lemma 1.0.12

Lemma 1.0.12. Assume $\zeta$ is a primitive element of $\mathbb{F}_{q^{n}}$ where $q$ is a prime power and $n \geq 2$. Given a function $F: \mathbb{F}_{q^{n}} \rightarrow \mathbb{F}_{q^{n}}$, define $f: \mathbb{Z}_{q^{n}-1} \rightarrow \mathbb{F}_{q^{n}}$ by $f(k)=F\left(\zeta^{k}\right)$ 
and let $r$ be the least period of $\mathcal{F}_{\zeta}[f]$ (which is the same as the least period of $\mathcal{F}_{\zeta}^{-1}[f]$ ). Then the following results hold.

(i) Suppose $r \nmid\left(q^{n}-1\right) / \Phi_{n}(q)$. Then $\operatorname{supp}(F)$ contains an element of degree $n$ over $\mathbb{F}_{q}$.

(ii) Suppose $\operatorname{supp}(F)$ contains an element of degree $n$ over $\mathbb{F}_{q}$. Then $r \nmid\left(q^{d}-1\right)$ for every positive divisor $d$ of $n$ with $d<n$.

(iii) Suppose $\operatorname{supp}(F)$ contains a primitive element of $\mathbb{F}_{q^{n}}$, or the least common multiple of the multiplicative orders of the elements in $\operatorname{supp}(F)$ equals $q^{n}-1$. Then $r=q^{n}-1$

In particular (i) implies the existence of an irreducible factor of degree $n$ for any polynomial $h(x) \in \mathbb{F}_{q}[x]$ satisfying a constraint on the least period as follows. Here $\mathbb{F}_{q^{n}}^{\times}$and $L^{\times}$denote the set of all invertible elements in $\mathbb{F}_{q^{n}}$ and $L$ respectively.

Lemma 1.0.13. Let $q$ be a prime power and $n \geq 2$. Suppose $h(x) \in \mathbb{F}_{q}[x]$ and $L$ is any subfield of $\mathbb{F}_{q^{n}}$ containing the image $h\left(\mathbb{F}_{q^{n}}^{\times}\right)$. Define the polynomial

$$
S(x)=\left(1-h(x)^{\# L^{\times}}\right) \bmod \left(x^{q^{n}-1}-1\right) \in \mathbb{F}_{q}[x]
$$

Write $S(x)=\sum_{i=0}^{q^{n}-2} s_{i} x^{i}$ for some coefficients $s_{i} \in \mathbb{F}_{q}$. If the cyclic sequence $\left(s_{i}\right)_{i=0}^{q^{n}-2}$ has least period $r$ satisfying $r \nmid\left(q^{n}-1\right) / \Phi_{n}(q)$, then $h(x)$ has an irreducible factor of degree $n$ over $\mathbb{F}_{q}$.

Note Lemma 1.0.13 immediately yields the following sufficient condition for a polynomial to be irreducible because $h(x)$ has an irreducible factor of the same degree.

Proposition 1.0.14. With the notations of Lemma 1.0.13, assume $h(x) \in \mathbb{F}_{q}[x]$ has degree $n \geq 2$. If the cyclic sequence $\left(s_{i}\right)_{i=0}^{q^{n}-2}$ of the coefficients of $S(x)$ has least period $r$ satisfying $r \nmid\left(q^{n}-1\right) / \Phi_{n}(q)$, then $h(x)$ is irreducible. 
To give the reader a flavor for the essence of our proof as an application of Lemma 1.0.13, we give the following small example.

Example 1.0.15. Let $q=2$, let $n=4$, and let

$$
\begin{aligned}
h(x) & =\sum_{0 \leq i_{1}<i_{2} \leq 3} x^{2^{i_{1}}+2^{i_{2}}} \\
& =x^{12}+x^{10}+x^{9}+x^{6}+x^{5}+x^{3} \in \mathbb{F}_{2}[x] .
\end{aligned}
$$

Note that $h\left(\mathbb{F}_{2^{4}}\right) \subseteq \mathbb{F}_{2}$. In fact, for any $\xi \in \mathbb{F}_{2^{4}}, h(\xi)$ is the coefficient of $x^{2}$ in the characteristic polynomial of degree 4 over $\mathbb{F}_{2}$ with root $\xi$. We may take $L=\mathbb{F}_{2}$ in Lemma 1.0.13; hence $\# L^{\times}=1$. Thus

$$
\begin{aligned}
S(x) & :=\left(1+h(x)^{\# L^{\times}}\right) \bmod \left(x^{2^{4}-1}+1\right)=h(x)+1 \\
& =x^{12}+x^{10}+x^{9}+x^{6}+x^{5}+x^{3}+1 \in \mathbb{F}_{2}[x] .
\end{aligned}
$$

The cyclic sequence of coefficients $\mathbf{s}=s_{0}, s_{1}, \ldots, s_{2^{4}-2}$ of $S(x)=\sum_{i=0}^{2^{4}-2} s_{i} x^{i}$ is given by

$$
\mathbf{s}=1,0,0,1,0,1,1,0,0,1,1,0,1,0,0 .
$$

One can easily check that the least period $r$ of $\mathbf{s}$ is $r=2^{4}-1$, the maximum possible. Because $2^{4}-1>\left(2^{4}-1\right) / \Phi_{4}(2)$, Lemma 1.0.13 implies that $h(x)$ has an irreducible factor $P(x)$ of degree 4 over $\mathbb{F}_{2}$. Any root $\xi$ of $P(x)$ must satisfy $h(\xi)=0$. This is the coefficient of $x^{2}$ in $P(x)$. Hence there exists an irreducible polynomial of degree 4 over $\mathbb{F}_{2}$ with its coefficient of $x^{2}$ being zero. Indeed, $x^{4}+x+1$ is one such irreducible polynomial.

The works mentioned above on irreducibles are primarily concerned with proving existence questions. As such, one finds it unnecessary to produce exact explicit 
formulas for the number of such irreducibles; estimates suffice. However there is also intensive research on the exact number of irreducibles or primitives with one or more prescribed coefficients. See for instance $[12,32,41-44,90]$ and the survey [17] or Section 3.5 by S. D. Cohen in the Handbook of finite fields [52]).

Let $\alpha$ be a primitive element of $\mathbb{F}_{q^{n}}$. For a positive divisor $N$ of $q^{n}-1$, we say that an element $\xi \in \mathbb{F}_{q^{n}}^{*}$ is $N$-free if $\xi=\alpha^{k}$ for some integer $k$ that is coprime to $N$. Note that the definition of $N$-free is independent of the choice of the primitive element $\alpha$. Clearly the primitive elements of $\mathbb{F}_{q^{n}}$ are exactly the $\left(q^{n}-1\right)$-free elements; thus the notion of $N$-free generalizes that of primitivity. Note as well the direct connection between the number of primitive elements in $\mathbb{F}_{q^{m}}$ with trace $c \in \mathbb{F}_{q}$ and number of primitive polynomials of degree $m$ over $\mathbb{F}_{q}$ having trace $-c$ : The latter equals the former divided by the degree, $m$.

In Chapter 6 we study the number of $N$-free elements, and in particular primitive elements, with prescribed trace. There we start by deriving a formula for the number of $N$-free elements over a finite field with prescribed trace, in particular trace zero, in terms of Gaussian periods. See Lemma 6.1.4 for this. Then, by using known results on Gaussian periods, we proceed to obtain several explicit expressions for these. See for instance Corollaries 1.0.19, 1.0.21, 1.0.22, 1.0.23, 1.0.25, 1.0.27. Motivated by a result of Carlitz [12] showing that the number of irreducibles polynomials of fixed degree, with prescribed non-zero trace, is uniform in the trace, we also consider the question of when do primitives satisfy a similar property. Although it could potentially be difficult to characterize all the possible cases when this holds, we give in Theorem 1.0.26 a sufficient criteria for which $N$-free elements, particularly primitives, satisfy this requirement. Finally we also explore the related number of elements in $\mathbb{F}_{q^{n}}^{*}$ with multiplicative order $N$ and having a prescribed trace.

Perhaps one of the earliest results, in the ennumeration of irreducible polynomials 
with prescribed coefficients, is the following beautiful formula due to Carlitz [12]. It describes the number of monic irreducible polynomials of degree $m$ with a prescribed trace coefficient (the coefficient of $x^{m-1}$ ). Let $I_{q, m}(c)$ denote the number of monic irreducible polynomials of degree $m$ over $\mathbb{F}_{q}$ with trace $c$, and let $\mu$ denote (as before) the Möbius function.

Theorem 1.0.16 (Carlitz (1952)). Let $q$ be a power of a prime $p$ and let $m \in$ $\mathbb{N}$. Then for any non-zero element $c \in \mathbb{F}_{q} \backslash\{0\}$, the number of monic irreducible polynomials of degree $m$ over $\mathbb{F}_{q}$ and with trace $c$ is given by

$$
I_{q, m}(c \neq 0)=\frac{1}{q m} \sum_{\substack{d \mid m \\ p \nmid d}} \mu(d) q^{m / d}=\frac{I_{q, m}-I_{q, m}(0)}{q-1}
$$

where

$$
I_{q, m}=\frac{1}{m} \sum_{d \mid m} \mu(d) q^{m / d}
$$

is the number of monic irreducible polynomials of degree $m$ over $\mathbb{F}_{q}$.

Note that

$$
I_{q, m}(c)=\frac{I_{q, m}-I_{q, m}(0)}{q-1}
$$

is a constant for any $c \in \mathbb{F}_{q}^{*}$, and so $I_{q, m}(c)$ is said to be uniformly distributed for $c \in \mathbb{F}_{q}^{*}$. One of the results of Chapter 6 concerns an analogy to (1.0.1) for primitive polynomials in some special cases. We will return to this concept later.

There is a correspondence between the primitive elements in $\mathbb{F}_{q^{m}}$ and the primitive polynomials of degree $m$ over $\mathbb{F}_{q}$. In fact the number of primitive elements in $\mathbb{F}_{q^{m}}$ is $m$ times the number of primitive polynomials of degree $m$ over $\mathbb{F}_{q}$. In the case of the primitive polynomials of degree $m$, or equivalently of primitive elements in $\mathbb{F}_{q^{m}}$, things are more complicated. Most of the work on primitive polynomials with prescribed 
coefficients focus on the asymptotic analysis for their number and existence. For example, the following existence result was first due to Cohen (see also [20] for a more self-contained proof). Here we use the notation $\operatorname{Tr}_{\mathbb{F}_{q^{m}} / \mathbb{F}_{q}}$ to denote the trace function from $\mathbb{F}_{q^{m}}$ onto $\mathbb{F}_{q}$.

Theorem 1.0.17 (Cohen (1990)). Let $q$ be a power of a prime and $m>1$ be a positive integer, and let $c$ be an arbitrary element in $\mathbb{F}_{q}$. If $m=2$ or $(q, m)=(4,3)$, further assume that $c \neq 0$. Then there exists a primitive element $\xi$ of $\mathbb{F}_{q^{m}}$ with $\operatorname{Tr}_{\mathbb{F}_{q} m / \mathbb{F}_{q}}(\xi)=c$.

One can see Section 4.2 by S. D. Cohen and the references therein, in the Handbook of Finite Fields [52]. In fact, except for the trivial cases and those when all the primitive polynomials of degree $m$ are all the irreducibles of degree $m$ (i.e., when $q=2$ and $m=\ell$ with $2^{\ell}-1$ a (Mersenne) prime) no explicit formulas are known to date. In particular an analogue, for primitives, to the formula (1.0.1) due to Carlitz is unknown, including in any specific non-trivial case of $q, m$.

Let $P_{q, m}$ be the number of primitive elements in $\mathbb{F}_{q^{m}}$. It is known that $P_{q, m}=$ $\phi\left(q^{m}-1\right)$, where $\phi$ is the Euler function. For $c \in \mathbb{F}_{q}$, let $P_{q, m}(c)$ denote the number of primitive elements in $\mathbb{F}_{q^{m}}$ with trace $c$. Then as a corollary of Theorem 1.0.26, the following result (analogous to (1.0.1)) is proved. For a positive integer $n$, let us denote with $\operatorname{Rad}(n)$ the product of all the distinct prime factors of $n$. By convention $\operatorname{Rad}(1)=1$.

Corollary of Theorem 1.0.26 Let $q$ be a prime power and $m$ be a multiple of $\operatorname{Rad}(q-1)$. Then, for $c \neq 0$, we have

$$
P_{q, m}(c)=\frac{P_{q, m}-P_{q, m}(0)}{q-1}
$$

Although the formula above corresponds to primitive elements and hence primitive 
polynomials, we will however work with the (more general) $N$-free elements. Let us first fix the following notations and definitions.

Notations: In what follows we let $q=p^{s}$ be a power of a prime number $p$, $m$ be a positive integer, $Q=\left(q^{m}-1\right) /(q-1)$, and $\alpha$ be a primitive element of $\mathbb{F}_{q^{m}}$. Furthermore, for an element $c \in \mathbb{F}_{q}$, we denote with $Z_{q, m, N}(c)$ the number of $N$-free elements $\xi$ in $\mathbb{F}_{q^{m}}^{*}$ such that $\operatorname{Tr}_{\mathbb{F}_{q} m / \mathbb{F}_{q}}(\xi)=c$. Moreover we let $P_{q, m, N}(c)$ be the number of non-zero elements $\zeta$ in $\mathbb{F}_{q^{m}}^{*}$ with multiplicative order $N$ and satisfying $\operatorname{Tr}_{\mathbb{F}_{q^{m} / \mathbb{F}_{q}}}(\zeta)=c$. In particular we note that

$$
Z_{q, m, q^{m}-1}(c)=P_{q, m, q^{m}-1}(c)=P_{q, m}(c)
$$

is the number of primitive elements $\xi$ in $\mathbb{F}_{q^{m}}$ such that $\operatorname{Tr}_{\mathbb{F}_{q^{m}} / \mathbb{F}_{q}}(\xi)=c$. For an integer $k$ and $N \mid q^{m}-1$, denote

$$
\Delta_{k}(N):=\sum_{d \mid N} \mu(d) \eta_{k}^{\left(d, q^{m}\right)},
$$

where in the sum $\eta_{k}^{\left(d, q^{m}\right)}$ is the $k$-th Gaussian period of type $\left(d, q^{m}\right)$ Note that the value of $\Delta_{k}(N)$ depends only on the square-free part of $N$.

Previously Cohen and Prešern [20] derived a formula for $Z_{q, m, N}(c)$ in terms of Gaussian sums (see Lemma 2.2 there). From this they were able to obtain lower bounds, through various assisting sieving inequalities, thus proving Theorem 1.0.17 in a more self-contained fashion than previously done in [16]. However as it was perhaps beyond the scope of their work, and except for their Corollary 2.3 where they give an explicit formula for $Z_{q, m, N}(c)$ in a few special cases of Corollary 1.0.23 and Corollary 1.0.27 below, their results were mainly constrained to lower bounds and existence results. It is interesting to note that in the case of trace zero, as they 
showed in their Lemma 2.1, there is the connection between primitives with trace zero and $Q$-free elements with trace zero: $Z_{q, m, q^{m}-1}(0)=\Theta(K) Z_{q, m, Q}(0)$. Here $K$ is the part of $q^{m}-1$ that is coprime to $Q$, and $\Theta(K)=\phi(K) / K$ is the proportion of primitive $K$-th roots of unity among the $K$-th roots. But more generally, as we show here thorough our calculations, a lemma due to Ding and Yang [26] (see Lemma 2.3.1 here) implies that something similar holds in general for any divisor $N$ of $q^{m}-1$ : $Z_{q, m, N}(0)=\Theta(K) Z_{q, m, \operatorname{gcd}(Q, N)}(0)$, where $K$ is now the part of $N$ that is coprime to $Q$. This follows from the following result.

Theorem 1.0.18. Let $q$ be a power of a prime and $m$ be a positive integer. Let $N \mid q^{m}-1$ and $K_{Q}$ be the largest divisor of $N$ that is coprime to $Q=\left(q^{m}-1\right) /(q-1)$. Then

$$
Z_{q, m, N}(0)=\frac{(q-1) \phi\left(K_{Q}\right)}{q K_{Q}}\left(\frac{Q}{\operatorname{gcd}(Q, N)} \phi(\operatorname{gcd}(Q, N))+\Delta_{0}(\operatorname{gcd}(Q, N))\right)
$$

Note that obtaining the value of $Z_{q, m, N}(0)$ boils down to computing $\Delta_{0}(\operatorname{gcd}(Q, N))$. Since Gaussian sums and hence periods are known in only very few cases, obtaining images of $\Delta_{0}$ may be quite hard in general. But by using known results on periods we can clearly obtain some explicit expressions. For instance we obtain the following two direct consequences when $\operatorname{gcd}(Q, N)=1$.

Corollary 1.0.19. Let $q$ be a power of a prime, let $m \in \mathbb{N}$ and let $N \mid q-1$ such that $N$ is coprime to $\left(q^{m}-1\right) /(q-1)$. Then the number of $N$-free elements $\xi \in \mathbb{F}_{q^{m}}$ with $\operatorname{Tr}_{\mathbb{F}_{q} m / \mathbb{F}_{q}}(\xi)=0$ is given by

$$
Z_{q, m, N}(0)=\frac{\phi(N)}{N}\left(q^{m-1}-1\right)
$$

Setting $N=1$ above one obtains the well-known number, $q^{m-1}-1$, of non-zero 
elements lying in the kernel of the trace map.

Corollary 1.0.20. Let $q$ be a power of a prime $p$ and assume that $Q=\left(q^{\ell}-1\right) /(q-1)$ is prime for some prime $\ell$. Then the number of primitive elements $\xi \in \mathbb{F}_{q^{\ell}}$ satisfying $\operatorname{Tr}_{\mathbb{F}_{q} \ell / \mathbb{F}_{q}}(\xi)=0$ is given by

$$
Z_{q, \ell, q^{\ell}-1}(0)= \begin{cases}\phi\left(q^{\ell}-1\right) / q & \text { if } \ell \neq p \\ \phi\left(q^{\ell}-1\right) / q-\phi(q-1) & \text { otherwise }\end{cases}
$$

Since quadratic and cubic Gaussian periods are known as well, we also immediately obtain Corollaries 1.0.21 and 1.0.22. These two correspond to the cases when $\operatorname{gcd}(Q, N)$ is a power of 2 and 3 , respectively.

Corollary 1.0.21. Let $q=p^{s}, Q=\left(q^{m}-1\right) /(q-1), N \mid q^{m}-1$ such that $\operatorname{gcd}(Q, N)=$ $2^{n}$ for some $n \geq 1$, and $K_{Q}$ be the largest odd divisor of $N$. Then

$$
\frac{2 q K_{Q}}{(q-1) \phi\left(K_{Q}\right)} Z_{q, m, N}(0)=\left\{\begin{array}{lll}
Q-1+(-1)^{s m} q^{m / 2} & \text { if } p \equiv 1 & (\bmod 4) \\
Q-1+(-\sqrt{-1})^{s m} q^{m / 2} & \text { if } p \equiv 3 & (\bmod 4)
\end{array}\right.
$$

Corollary 1.0.22. Let $p \equiv 1(\bmod 3)$, let $q=p^{s}, Q=\left(q^{m}-1\right) /(q-1), N \mid q^{m}-1$ such that $\operatorname{gcd}(N, Q)=3^{n}$ for some $n \geq 1$, and $K_{Q}$ be the largest divisor of $N$ with $3 \nmid K_{Q}$. Let $c \in \mathbb{Z}$ with $c \equiv 1(\bmod 3)$ and $p \nmid c$ be a solution to the equation $4 q^{m / 3}=c^{2}+27 d^{2}$ with $d \in \mathbb{Z}$. Then

$$
Z_{q, m, N}(0)=\frac{(q-1) \phi\left(K_{Q}\right)}{q K_{Q}}\left(\frac{2 Q-2-c q^{m / 3}}{3}\right) .
$$

Thanks in part to well-known explicit expressions for the Gaussian periods in the so called semi-primitive case (see Lemma 2.3.6), we obtain the following result. 
Corollary 1.0.23. Let $s m$ be even with $m>1, q=p^{s}$ be a power of a prime $p$, and $Q=\left(q^{m}-1\right) /(q-1)$. Let $N \mid q^{m}-1$ be such that $n:=\operatorname{gcd}(Q, N)>1$ is not a power of 2 . Further assume there exists a positive integer $j$ such that $p^{j} \equiv-1(\bmod \ell)$ for every prime divisor $\ell \geq 3$ of $n$, and that $j$ is the least such. Define $\gamma=s m / 2 j$. Let $K_{Q}$ be the part of $N$ that is coprime to $Q$. Let $\eta_{0}^{\left(2, q^{m}\right)}$ be as in Lemma 2.3.4. Then the number of $N$-free elements $\xi \in \mathbb{F}_{q^{m}}$ with $\operatorname{Tr}_{\mathbb{F}_{q} m / \mathbb{F}_{q}}(\xi)=0$ is given by

$$
Z_{q, m, N}(0)=\frac{(q-1) \phi\left(K_{Q}\right)}{q K_{Q}}\left(\frac{Q}{n} \phi(n)+\Delta_{0}(n)\right)
$$

where the value of $\Delta_{0}(n)$ is given in what follows.

(a) If $\gamma$ and $p$ are odd, $n$ is even and $\left(p^{j}+1\right) / 2$ is odd, then

$$
\Delta_{0}(n)=-\eta_{0}^{\left(2, q^{m}\right)}-\left(1+q^{m / 2}\right)\left(\frac{1}{2}+\frac{\phi(n)}{n}\right)
$$

(b) In all other cases,

$$
\Delta_{0}(n)=-\epsilon_{2} \cdot\left(\frac{(-1)^{\gamma} q^{m / 2}+1}{2}+\eta_{0}^{\left(2, q^{m}\right)}\right)+\frac{(-1)^{\gamma} q^{m / 2}-1}{n} \phi(n),
$$

where

$$
\epsilon_{2}= \begin{cases}1 & \text { if } n \text { is even } \\ 0 & \text { otherwise }\end{cases}
$$

It is a simple matter to show that in the case of quadratic extensions $(m=2)$ no primitive element of $\mathbb{F}_{q^{2}}$ with trace zero, in $\mathbb{F}_{q}$, exists. In fact, this case falls under the more general category below of Proposition 1.0.24, for which we are able to, in Chapter 6, obtain the corresponding formula for the zero trace.

Proposition 1.0.24. Let $q=p^{s}$ be a power of a prime number $p$ and let $m>1$ be 
an integer. Then there exists a positive integer $j$ such that

$$
p^{j} \equiv-1 \quad\left(\bmod \operatorname{Rad}\left(\frac{q^{m}-1}{q-1}\right)\right)
$$

if and only if $m=2$, or $q=p$ is a Mersenne prime and $m=4$. The latter case holds precisely for every $j=2 k$ with $k \geq 1$ odd.

Recall that a Mersenne prime $M_{\ell}$ is of the form $M_{\ell}=2^{\ell}-1$ for some prime $\ell$. Usually the world's record of the largest prime is broken by a Mersenne prime, and, although only 48 such primes have been discovered thus far (see the Great Internet Mersenne Prime Search (GIMPS) available online) it is a well-known conjecture that there exist infinitely many of them. They appear in various areas of number theory and finite fields, including in the Great Trinomial Hunt [9], an ongoing project for the search of primitive trinomials (i.e., primitive polynomials with exactly three non-zero terms) over $\mathbb{F}_{2}$ with degree the "exponent" $\ell$ of a Mersenne prime $M_{\ell}$.

We obtain the following simple formula for the number of primitive elements, with absolute trace zero, in quartic extensions of Mersenne prime fields.

Corollary 1.0.25. Let $p$ be a Mersenne prime. Then the number of primitive elements $\xi$ in $\mathbb{F}_{p^{4}}$ satisfying $\operatorname{Tr}_{\mathbb{F}_{p^{4}} / \mathbb{F}_{p}}(\xi)=0$ is given by

$$
\frac{1}{p}\left(\phi\left(p^{4}-1\right)-\phi\left(\frac{p^{4}-1}{p+1}\right)\right) .
$$

In Chapter 6 we also discuss the concept of uniformity, already met in Theorem 1.0.16, now for $N$-free elements; in particular, for primitive elements. Although it is easy to find examples of $q, m, N$ for which $Z_{q, m, N}(c)$ does not behave uniformly for $c \in \mathbb{F}_{q}^{*}$ (and indeed, when $N=q^{m}-1$ is fixed as well) it is of special interest to find and classify instances of $q, m, N$ for which $Z_{q, m, N}(c \neq 0)$ does. One of the obvious 
reasons being that, in this case, in order to obtain the number of $N$-free elements with a prescribed non-zero trace, it would be enough to find the corresponding number for the zero trace. The following theorem gives a sufficient criteria for this to happen, but we ask the interested able reader to characterize all such instances of $q, m, N$.

Theorem 1.0.26. Let $q$ be a power of a prime and $N$ be a positive divisor of $q^{m}-1$. If every prime divisor of $N$ divides $Q=\left(q^{m}-1\right) /(q-1)$, then, for every element $c \in \mathbb{F}_{q} \backslash\{0\}$, the number of $N$-free elements $\xi \in \mathbb{F}_{q^{m}}$ satisfying $\operatorname{Tr}_{\mathbb{F}_{q} / \mathbb{F}_{q}}(\xi)=c$ is given by

$$
Z_{q, m, N}(c \neq 0)=\frac{\frac{q^{m}-1}{N} \phi(N)-Z_{q, m, N}(0)}{q-1}
$$

In particular, setting $N=q^{m}-1$, we obtain that $Z_{q, m, q^{m}-1}(c)$ is a constant independent of $c \in \mathbb{F}_{q}^{*}$ whenever the radical (the product of all the distinct prime divisors) of $Q$ is the same as that of $q^{m}-1$. This occurs exactly when all the prime factors of $q-1$ divide $m$; see Corollary 6.3.3 for this. Thus we obtain the following immediate consequence to Theorem 1.0.26 and Corollary 1.0.23.

Corollary 1.0.27. Assume that $q, r, N$, satisfy the assumptions of Corollary 1.0.23 and further assume that $\operatorname{Rad}(N) \mid Q$. Let $\eta_{0}^{\left(2, q^{m}\right)}$ be as in Lemma 2.3.4. Then for any non-zero $c \in \mathbb{F}_{q}^{*}$, the number of $N$-free elements $\xi \in \mathbb{F}_{q^{m}}$ with $\operatorname{Tr}_{\mathbb{F}_{q^{m}} / \mathbb{F}_{q}}(\xi)=c$ is given in what follows.

(a) If $\gamma$ and $p$ are odd, $N$ is even and $\left(p^{j}+1\right) / 2$ is odd, then

$$
Z_{q, m, N}(c \neq 0)=\frac{1}{q}\left(\eta_{0}^{\left(2, q^{m}\right)}+\left(1+q^{m / 2}\right)\left(\frac{1}{2}+\frac{\phi(N)}{N}\right)+\phi(N)\left(\frac{q Q}{N}-1\right)\right)
$$

(b) In all other cases,

$$
Z_{q, m, N}(c \neq 0)=\frac{\phi(N)}{q N}\left(q^{m}+(-1)^{\gamma+1} q^{m / 2}+q Q-N+\epsilon_{2} \cdot\left(\frac{(-1)^{\gamma} q^{m / 2}+1}{2}+\eta_{0}^{\left(2, q^{m}\right)}\right)\right)
$$


where

$$
\epsilon_{2}= \begin{cases}1 & \text { if } N \text { is even } \\ 0 & \text { otherwise }\end{cases}
$$

As a consequence of Theorem 1.0.26 one obtains the following interesting property of the sum $\Delta_{0}(N)$ for $N \mid q^{m}-1$ such that $\operatorname{Rad}(N) \mid Q=\left(q^{m}-1\right) /(q-1)$. It is the constant difference between the number of $N$-free elements with zero and non-zero traces in $\mathbb{F}_{t}$, for any subfield $\mathbb{F}_{t}$ of $\mathbb{F}_{q}$.

Corollary 1.0.28. Let $N \mid q^{m}-1$ such that every prime divisor of $N$ divides $\left(q^{m}-\right.$ $1) /(q-1)$. Then for every subfield $\mathbb{F}_{t}$ of $\mathbb{F}_{q}$ and every $c_{t} \in \mathbb{F}_{t}^{*}$, we have

$$
\Delta_{0}(N)=Z_{t, m\left[\mathbb{F}_{q}: \mathbb{F}_{t}\right], N}(0)-Z_{t, m\left[\mathbb{F}_{q}: \mathbb{F}_{t}\right], N}\left(c_{t} \neq 0\right)
$$

Furthermore, if $t \neq q$, then

$$
\Delta_{0}(N)=\frac{q Z_{q, m, N}(0)-t Z_{t, m\left[\mathbb{F}_{q}: \mathbb{F}_{t}\right], N}(0)}{q-t} .
$$

Although the work is primarily concerned with the number $Z_{q, m, N}(c)$, we also briefly consider the seemingly closely related number, $P_{q, m, N}(c)$, of elements with order $N$ having a prescribed trace $c$. There we derive a general formula for $P_{q, m, N}(c)$ (see Lemma 6.4.3) and show its relation to $Z_{q, m, N}(c)$ as well as Hamming weights of specific codewords in irreducible cyclic codes. Let $L_{Q}$ be the largest divisor of $q^{m}-1$ with the same radical as that of $Q$. We also show, in Lemma 6.4.3, that if $N \mid q^{m}-1$ is such that $L_{Q} \mid N$, then the following simple relation holds: $Z_{q, m, N}(0)=\frac{q^{m}-1}{N} P_{q, m, N}(0)$. We believe it should not be too difficult to generalize this even further for arbitrary $N$, but we leave this to the interested reader. As a consequence of this and of Cohen's result (see Theorem 1.0.17 above) we show in Theorem 6.4.4 that there exists an element in 
$\mathbb{F}_{q^{m}}^{*}$ of order $N$ (with $\left.L_{Q} \mid N\right)$ having trace 0 if and only if $m \neq 2$ and $(q, m) \neq(4,3)$. In addition Lemma 6.4.3 together with Corollary 1.0.18 yields, in Theorem 6.4.5, an explicit expression for the number of elements of order $2(p+1)\left(p^{2}+1\right)$, in quartic extensions of Mersenne prime fields $\mathbb{F}_{p}$, having absolute trace zero.

We now briefly summarize and recall the content of each chapter. In Chapter 2 we review some preliminary facts and concepts which will be of use in subsequent chapters. These include compositional inverses of linearized polynomials bijecting subspaces, Gaussian sums and periods, as well as the DFT for finite-field-valued functions, period of functions and cyclotomic polynomials. In Chapter 3 we study the compositional inverses of several classes of PPs, while in Chapter 4 we focus on the topic of CPPs and obtain several results in this area. In Chapter 5 we consider existence of irreducibles with a prescribed coefficient and give a new proof of the Hansen-Mullen irreducibility conjecture. Finally in Chapter 6 we work on the ennumeration of $N$-free and primitive elements with restrictions on their trace. 


\section{Chapter 2}

\section{Preliminaries}

In this chapter we go over some preliminary facts which will be of use later in the chapters that follow. The content of this chapter is as follows. In Section 2.1 we study the compositional inverses of linearized polynomials inducing bijections of subspaces. Worth pointing out here is Theorem 2.1.5, where we show how to obtain such compositional inverses on subspaces. As we show there, obtaining the coefficients of such linearized polynomials is equivalent to solving a system of linear equations. It is also important to point out Lemma 2.1.10 there, which will be quite useful in Chapter 3 to obtain the compositional inverses of several classes of PPs. In Section 2.2 we introduce several basic concepts regarding the DFT, period of functions and cyclotomic polynomials. We then conclude this chapter with Section 2.3, where we define objects such as Gaussian sums and periods, and gather from literature some known explicit formulas for the Gaussian periods. These will be of use later in Chapter 6 . 


\subsection{Inverses of linearized polynomials inducing bi- jections of subspaces}

Linearized polynomials play a crucial role throughout the work ${ }^{1}$ in Chapters 3,4 and we thus explore here some of their properties. We recall that we defined $L(x) \in \mathbb{F}_{q^{n}}[x]$ to be linearized, also called a $q$-polynomial, if $L(x)=\sum_{i=0}^{m} a_{i} x^{q^{i}}$ for some $a_{i} \in \mathbb{F}_{q^{n}}$. We are mainly interested in these from the point of view of a mapping on $\mathbb{F}_{q^{n}}$, and so we assume here that $m=n-1$.

One can show that all the additive maps of $\mathbb{F}_{q^{n}}$ are induced by $p$-polynomials. Clearly, $q$-polynomials are also $p$-polynomials. The set, $\mathscr{L}_{n}\left(\mathbb{F}_{q^{n}}\right)$, of all $q$-polynomials over $\mathbb{F}_{q^{n}}$, forms a unital associative non-commutative $\mathbb{F}_{q^{-}}$algebra with multiplication given by the composition of polynomials, and using the usual addition of polynomials and scalar multiplication by elements of $\mathbb{F}_{q}$. In contrast, its subset of all $q$-polynomials over $\mathbb{F}_{q}$ does form a commutative subalgebra. Even more, $q$-polynomials over $\mathbb{F}_{p}$ commute with $p$-polynomials over $\mathbb{F}_{q}$. For a $q$-polynomial $L(x)=\sum_{i=0}^{n-1} a_{i} x^{q^{i}}, a_{i} \in \mathbb{F}_{q^{n}}$, we associate its so called Dickson matrix $D_{L}$ given by

$$
D_{L}=\left(\begin{array}{cccc}
a_{0} & a_{1} & \cdots & a_{n-1} \\
a_{n-1}^{q} & a_{0}^{q} & \cdots & a_{n-2}^{q} \\
\vdots & \vdots & & \vdots \\
a_{1}^{q^{n-1}} & a_{2}^{q^{n-1}} & \cdots & a_{0}^{q^{n-1}}
\end{array}\right)
$$

It is well-known that $L$ is a permutation polynomial of $\mathbb{F}_{q^{n}}$ if and only if $D_{L}$ is non-

\footnotetext{
${ }^{1}$ This section is substantively based on some of the content of the following article originally published in Finite Fields and Their Applications, https ://doi.org/10.1016/j.ffa.2014.02.006. It is released under a Creative Commons Attribution Non-Commercial No Derivatives License.

[70] A. Tuxanidy and Q. Wang, On the inverses of some classes of permutations of finite fields, Finite Fields and their Applications, v.28 (2014), p.244-281. https://doi.org/10.1016/j.ffa. 2014.02 .006
} 
singular [48]. Note also that $\mathscr{L}_{n}\left(\mathbb{F}_{q^{n}}\right)$ is isomorphic to the algebras of the $n \times n$ matrices over $\mathbb{F}_{q}$, and the $n \times n$ Dickson matrices over $\mathbb{F}_{q^{n}}$. In particular, the subset of $q$-polynomial permutations over $\mathbb{F}_{q^{n}}$ forms a group, under composition, that is isomorphic to the group of non-singular $n \times n$ matrices over $\mathbb{F}_{q}$. See the recent work in [80] for known and new characterizations of $\mathscr{L}_{n}\left(\mathbb{F}_{q^{n}}\right)$. It will also be useful to add the basic fact that a $q$-polynomial $L$ over $\mathbb{F}_{q}$ permutes $\mathbb{F}_{q}$ if and only if $L(1) \neq 0$, since $L(x)=L(1) x$ for all $x \in \mathbb{F}_{q}$.

In Chapter 3 we shall give expressions for the compositional inverses, or preimages in certain cases, particularly written in terms of linearized polynomials inducing the inverse map over subspaces on which prescribed linearized polynomials induce a bijection. Thus here in this section we show, in Theorem 2.1.5, how to obtain such linearized inverses over subspaces. See Lemma 2.1.4 as well. Note however that in the case when $L(x)$ is a $\mathrm{PP}$ of $\mathbb{F}_{q^{n}}$, the following result from [80] gives its compositional inverse in terms of cofactors of its associate Dickson matrix. We are however interested in deriving the inverse map of linearized polynomials in the case when these induce bijections of subspaces.

Proposition 2.1.1 (Theorem 4.8, [80]). Let $L(x)=\sum_{i=0}^{n-1} a_{i} x^{q^{i}} \in \mathscr{L}_{n}\left(\mathbb{F}_{q^{n}}\right)$ be a linearized permutation polynomial and $D_{L}$ be its associate Dickson matrix. Assume $\bar{a}_{i}$ is the $(i, 0)$-th cofactor of $D_{L}, 0 \leq i \leq n-1$. Then $\operatorname{det}(L)=\sum_{i=0}^{n-1} a_{n-i}^{q^{i}} \bar{a}_{i}$ and

$$
L^{-1}(x)=\frac{1}{\operatorname{det}(L)} \sum_{i=0}^{n-1} \bar{a}_{i} x^{q^{i}}
$$

We start off by briefly noting a few basic properties of the set $S_{\psi}:=\{x-\psi(x) \mid x \in$ $\left.\mathbb{F}_{q^{n}}\right\}$, which will appear often throughout the work in Chapter 3. For $\psi \in \mathscr{L}_{n}\left(\mathbb{F}_{q^{n}}\right)$, we denote by $\psi^{i}$ the compositional power of $\psi$, that is, $\psi^{i}=\psi \circ \psi \circ \cdots \circ \psi, i$ times. 
Denote by id $\in \mathscr{L}_{n}\left(\mathbb{F}_{q^{n}}\right)$ the identity $\operatorname{id}(x)=x$.

Lemma 2.1.2. Let $\psi \in \mathscr{L}_{n}\left(\mathbb{F}_{q^{n}}\right)$. Then the set $S_{\psi}:=\left\{x-\psi(x) \mid x \in \mathbb{F}_{q^{n}}\right\}$ is an

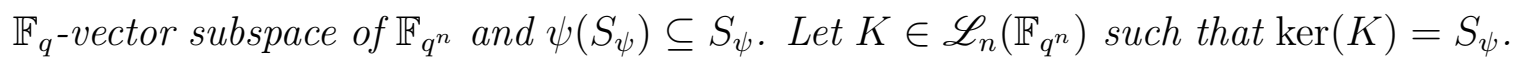
Then, for all $i \geq 0, K=K \circ \psi^{i}$ and $\operatorname{ker}\left(\psi^{i}\right) \subseteq S_{\psi}$. If $\psi^{l}=\psi^{l+1}$ for some positive integer $l$, then $S_{\psi}=\operatorname{ker}\left(\psi^{l}\right)$.

Proof. The reader can check that $S_{\psi}$ is indeed a vector space over $\mathbb{F}_{q}$ and $\psi\left(S_{\psi}\right) \subseteq S_{\psi}$. Since $\operatorname{ker}(K)=\operatorname{im}(\mathrm{id}-\psi)$, then for all $x \in \mathbb{F}_{q^{n}}$ we have $K \circ(\mathrm{id}-\psi)(x)=0$ and thus $K(x)=K(\psi(x))=K(\psi(\psi(x)))=\cdots$, i.e., $K=K \circ \psi^{i}$ for each $i \geq 0$. If $x \in \operatorname{ker}\left(\psi^{i}\right)$, then $K(x)=K\left(\psi^{i}(x)\right)=K(0)=0$. Hence $\operatorname{ker}\left(\psi^{i}\right) \subseteq \operatorname{ker}(K)=S_{\psi}$. If $\psi^{l}=\psi^{l+1}$, then $S_{\psi} \subseteq \operatorname{ker}\left(\psi^{l}\right)$; it follows that $S_{\psi}=\operatorname{ker}\left(\psi^{l}\right)$.

In particular, if $\psi$ is idempotent, i.e., $\psi^{2}=\psi$, then $S_{\psi}=\operatorname{ker}(\psi)$. Moreover, if $\psi$ is nilpotent, i.e., there exists a positive integer $l$ such that $\psi^{l}=0$, then $S_{\psi}=\operatorname{ker}(0)=$ $\mathbb{F}_{q^{n}}$

Let $v_{q, n}: \mathscr{L}_{n}\left(\mathbb{F}_{q^{n}}\right) \rightarrow \mathbb{F}_{q^{n}}^{n}$ be the natural map defined by

$$
v_{q, n}\left(\sum_{i=0}^{n-1} a_{i} x^{q^{i}}\right)=\left(\begin{array}{llll}
a_{0} & a_{1} & \cdots & a_{n-1}
\end{array}\right)
$$

(for simplicity we write vectors horizontally). Let $X=\left(x, x^{q}, \ldots, x^{q^{n-1}}\right)$ and let $X^{t}$ denote the transpose of $X$. The following lemma shows that the composition of two linearized polynomials is essentially induced via a Dickson matrix multiplication.

Lemma 2.1.3. Let $\varphi, \psi \in \mathscr{L}_{n}\left(\mathbb{F}_{q^{n}}\right)$, let $D_{\psi}$ be the Dickson matrix corresponding to $\psi$. Then $\varphi(\psi(x))=v_{q, n}(\varphi) D_{\psi} X^{t}$.

Proof. Write $v_{q, n}(\varphi)=\left(a_{0} a_{1} \cdots a_{n-1}\right), v_{q, n}(\psi)=\left(b_{0} b_{1} \cdots b_{n-1}\right)$, for some coefficients 
$a_{i}, b_{j} \in \mathbb{F}_{q^{n}}$. Then

$$
\begin{aligned}
\varphi(\psi(x)) & =\sum_{i=0}^{n-1} a_{i}\left(\sum_{j=0}^{n-1} b_{j} x^{q^{j}}\right)^{q^{i}}=\sum_{i=0}^{n-1} \sum_{j=0}^{n-1} a_{i} b_{j}^{q^{i}} x^{q^{i+j}}=\sum_{i=0}^{n-1} \sum_{k=0}^{n-1} a_{i} b_{k-i}^{q^{i}} x^{q^{k}} \\
& =\sum_{k=0}^{n-1} \sum_{i=0}^{n-1} a_{i} b_{k-i}^{q^{i}} x^{q^{k}}
\end{aligned}
$$

where the subscripts are reduced modulo $n$. Since the $i k$-th entry of $D_{\psi}$ is given by $b_{k-i}^{q^{i}}$, the result follows.

We recall the well known concept of a projection operator on a vector space. These are the idempotent linear transformations. Let $U$ be a vector space which we write as the internal direct sum of two subspaces $V, W$, that is, $U=V \oplus W$, where $U=V+W$ and $V \cap W=\{0\}$. We can write any element $u \in U$ uniquely as a sum $u=v+w$ where $v \in V$ and $w \in W$. Then the map $P: U \rightarrow U$ defined by $P(v+w)=w$ is called a projection operator of $U$ onto $W$ along $V$. It is easy to see that $P$ is a well-defined linear transformation with kernel $V$ and image $W$, and that $P(u)=u$ if and only if $u \in W$. In fact, $P$ is a projection operator if and only if it is idempotent, i.e., $P(P(u))=P(u)$ for all $u \in U$. Note that the existence of a projection operator onto any subspace is guaranteed, and thus we can always find at least one idempotent with a prescribed kernel.

The following lemma gives the inverse map over subspaces on which a prescribed linear transformation is a bijection. It is true in general for arbitrary vector spaces and linear transformations.

Lemma 2.1.4. Let $V, \bar{V}$, be two subspaces of a vector space $U$ over a field, each of the same dimension, and let the linear operator $\varphi: U \rightarrow U$ be bijective from $V$ to $\bar{V}$. There exists an idempotent $K: U \rightarrow U$ such that $\operatorname{ker}(K)=V$. Define the linear transformation $L: \varphi(U) \rightarrow V$ by $L(\varphi(u))=u-K(u)$. Then $\left.L\right|_{\bar{V}}$ is the inverse map 
of $\left.\varphi\right|_{V}$.

Proof. Since we can construct a projection operator with kernel $V$, it is clear that such an idempotent $K$ exists. First note that since $V=\operatorname{ker}(K)=\{u-K(u) \mid u \in U\}$, then $L(\varphi(U))=V$. To show that $L$ is well-defined, assume that $\varphi(u)=\varphi(w)$ and $u \neq w$ (the case $u=w$ is trivial). We need to show that $L(\varphi(u))=L(\varphi(w))$. Because $\varphi$ is injective on $\operatorname{ker}(K)$, then $\operatorname{ker}(\varphi) \cap \operatorname{ker}(K)=\{0\}$; thus, since $w-u \in \operatorname{ker}(\varphi)$, necessarily $w-u \notin \operatorname{ker}(K)$, otherwise $u=w$, a contradiction. Since $K$ is idempotent, it is a projection operator of $U$ onto $K(U)$ along $\operatorname{ker}(K)$, and thus $U=\operatorname{ker}(K) \oplus K(U)$. This means that $\operatorname{ker}(K) \cap K(U)=\{0\}$ and $\operatorname{dim}(U)=\operatorname{dim}(\operatorname{ker}(K))+\operatorname{dim}(K(U))$ implying $U=\operatorname{ker}(K) \cup K(U)$. Now, since $w-u \notin \operatorname{ker}(K)$, necessarily $w-u \in K(U) \backslash\{0\}$, say $w-u=K(z)$ for some $z \in U \backslash \operatorname{ker}(K)$. Then $K(w-u)=K(K(z))=K(z)=w-u$. Hence $L(\varphi(w))-L(\varphi(u))=w-u-K(w-u)=0$ implying that $L$ is well-defined. The reader can check that $L$ is a linear transformation. To see that $\left.L\right|_{\bar{V}}=\left.\varphi^{-1}\right|_{\bar{V}}$, note that for all $v \in V=\operatorname{ker}(K), L(\varphi(v))=v-K(v)=v$, from which we get $\varphi(L(\varphi(v)))=\varphi(v)$. Since $\varphi(V)=\bar{V}$, this is the same as $\varphi(L(\bar{v}))=\bar{v}$ for all $\bar{v} \in \bar{V}$. Then $\left.L\right|_{\bar{V}}=\left.\varphi^{-1}\right|_{\bar{V}}$ by the uniqueness of inverse maps.

We can now apply Lemma 2.1.3 and 2.1.4 to obtain linearized polynomials inducing the inverse map over subspaces on which a given linearized polynomial induces a bijection. Obtaining the coefficients of such linearized polynomials is equivalent to solving a system of linear equations.

Theorem 2.1.5. Let $V, \bar{V}$, be two equally sized $\mathbb{F}_{q^{-}}$subspaces of $\mathbb{F}_{q^{n}}$, let $\varphi \in \mathscr{L}_{n}\left(\mathbb{F}_{q^{n}}\right)$ induce a bijection from $V$ to $\bar{V}$, and let $D_{\varphi}$ be the associate Dickson matrix of $\varphi$. There exists $K \in \mathscr{L}_{n}\left(\mathbb{F}_{q^{n}}\right)$ inducing an idempotent endomorphism of $\mathbb{F}_{q^{n}}$ such that $\operatorname{ker}(K)=$ $V$. Then the coefficients, $\bar{c}=\left(\begin{array}{lll}c_{0} & c_{1} \cdots c_{n-1}\end{array}\right)$, of one of the linearized polynomials inducing the inverse map of $\left.\varphi\right|_{V}$, satisfy the linear equation $\bar{c} D_{\varphi}=v_{q, n}(\mathrm{id}-K)$. 
Proof. We make use of the fact that when $U=\mathbb{F}_{q^{n}}$ in Lemma 2.1.4, all the linear transformations are induced by linearized polynomials. Now define the map $\bar{L}$ : $\mathbb{F}_{q^{n}} \rightarrow \mathbb{F}_{q^{n}}$ by $\bar{L}=L \circ P$, where $L$ is given in Lemma 2.1.4 (with $U=\mathbb{F}_{q^{n}}$ ), and $P$ is a projection operator of $\mathbb{F}_{q^{n}}$ onto $\varphi\left(\mathbb{F}_{q^{n}}\right)$. Note that $\bar{L}$ is a well-defined linear operator. The fact that $P$ is a projection operator onto $\varphi\left(\mathbb{F}_{q^{n}}\right)$ gives $\bar{L}(\varphi(x))=$ $L \circ P(\varphi(x))=L(\varphi(x))=x-K(x)$. In particular, $\left.\bar{L}\right|_{\bar{V}}=\left.L\right|_{\bar{V}}=\left.\varphi^{-1}\right|_{\bar{V}}$. Thus we have obtained a linear operator of $\mathbb{F}_{q^{n}}$ inducing the inverse map of $\left.\varphi\right|_{V}$. But all linear operations of $\mathbb{F}_{q^{n}}$ are induced by linearized polynomials. Since, additionally, $V, \bar{V}$, are $\mathbb{F}_{q^{-}}$-subspaces and $\varphi \in \mathscr{L}_{n}\left(\mathbb{F}_{q^{n}}\right)$, it follows that the map of $\bar{L}$ can be induced by a linearized polynomial in $\mathscr{L}_{n}\left(\mathbb{F}_{q^{n}}\right)$, say $R$, inducing the inverse map of $\left.\varphi\right|_{V}$, and necessarily satisfying the equation $R(\varphi(x))=x-K(x)$. Then using Lemma 2.1.3 we obtain the linear equation $v_{q, n}(R) D_{\varphi}=v_{q, n}(\mathrm{id}-K)$ (after cancelling out the arbitrary $X^{t}$ on both sides) as required.

Note that if $\varphi$ induces a permutation of $\mathbb{F}_{q^{n}}$, i.e., $D_{\varphi}$ is non-singular, then the required idempotent is $K=0$, and the coefficients of the compositional inverse of $\varphi$ are given by $\bar{c}=v_{q, n}(\mathrm{id}) D_{\varphi}^{-1}$, i.e., the coefficients in the first row of $D_{\varphi}^{-1}$. See (1) in the Introduction and Proposition 2.1.1 for details.

Remark 2.1.6. In the case that the coefficients of $\varphi \in \mathscr{L}_{n}\left(\mathbb{F}_{q^{n}}\right)$ belong to $\mathbb{F}_{q}, D_{\varphi}$ becomes a circulant matrix. Then if $V$ is an $\mathbb{F}_{q}$-subspace and if the characteristic $p$ of $\mathbb{F}_{q^{n}}$ does not divide $n$, we can quickly solve the linear equation $\bar{c} D_{\varphi}=v_{q, n}(\mathrm{id}-K)$ by using the Circular Convolution Theorem for DFTs together with an FFT. See [87] for details on circulants and DFTs.

An immediate consequence is the following.

Corollary 2.1.7. Let $\varphi, \psi \in \mathscr{L}_{n}\left(\mathbb{F}_{q^{n}}\right)$ such that $\psi$ induces an idempotent endomorphism of $\mathbb{F}_{q^{n}}$ and $\varphi$ induces a bijection from $S_{\psi}=\operatorname{ker}(\psi)$ to an $\mathbb{F}_{q^{-}}$-subspace $\bar{V}$ of 
$\mathbb{F}_{q^{n}}$. Then the coefficients, $\bar{c}=\left(c_{0} c_{1} \cdots c_{n-1}\right)$, of a linearized polynomial inducing the inverse map from $\bar{V}$ to $S_{\psi}$, satisfy the linear equation $\bar{c} D_{\varphi}=v_{q, n}(\mathrm{id}-\psi)$.

Example 2.1.8. Assume that the characteristic $p$ does not divide $n$ and work over $\mathbb{F}_{q^{n}}$, where $q=p^{m}$ as before. We have $n^{-1} T\left(n^{-1} T(x)\right)=n^{-2} T^{2}(x)=n^{-2} n T(x)=$ $n^{-1} T(x)$ and so $n^{-1} T$ is idempotent with kernel $\operatorname{ker}(T)=\left\{\beta^{q}-\beta \mid \beta \in \mathbb{F}_{q^{n}}\right\}$. Let $\varphi(x):=x^{p}+c x \in \mathscr{L}_{m n}\left(\mathbb{F}_{p^{m n}}\right)$, where $c \in \mathbb{F}_{q}^{*}$ satisfies $c^{(q-1) /(p-1)}=(-1)^{m}$, and let $D_{\varphi}$ be its associate $m n \times m n$ Dickson matrix given by

$$
D_{\varphi}=\left(\begin{array}{cccccc}
c & 1 & 0 & 0 & \cdots & 0 \\
0 & c^{p} & 1 & 0 & \cdots & 0 \\
\vdots & \vdots & & & \vdots \\
1 & 0 & 0 & 0 & \cdots & c^{p^{m n-1}}
\end{array}\right)
$$

Note that $\varphi(\operatorname{ker}(T)) \subseteq \operatorname{ker}(T)$. If $\varphi$ permutes the $\mathbb{F}_{p}$-subspace, $\operatorname{ker}(T)$, of $\mathbb{F}_{q^{n}}$, then by Theorem 2.1.5, the coefficients, $\bar{d}=\left(d_{0} d_{1} \cdots d_{m n-1}\right)$, of one of the linearized polynomials inducing the inverse permutation of $\left.\varphi\right|_{\operatorname{ker}(T)}$, are a solution to the linear equation

$$
\begin{aligned}
\bar{d} D_{\varphi} & =v_{p, m n}\left(\mathrm{id}-n^{-1} T\right)=v_{p, m n}\left(x-n^{-1} \sum_{k=0}^{n-1} x^{p^{k m}}\right) \\
& =-n^{-1}(1-n, 0,0, \ldots, 0,1,0,0, \ldots, 0,1,0,0, \ldots, 0),
\end{aligned}
$$

where the 1 entries occur at indices (which start at 0 and end at $m n-1$ ) that are non-zero multiples of $m$. Solving this system we obtain a solution

$$
d_{k m+j}=n^{-1}(-1)^{j} c^{-\left(p^{j+1}-1\right) /(p-1)}(n-1-k),
$$

where $0 \leq k \leq n-1$ and $0 \leq j \leq m-1$. Thus we have found a linearized polynomial 
inducing the inverse map of $\left.\varphi\right|_{\operatorname{ker}(T)}$. In particular, $\varphi$ induces a permutation of $\operatorname{ker}(T)$ as maps are invertible if and only if they are bijections.

Remark 2.1.9. There exist several linearized polynomials inducing idempotents operations of $\mathbb{F}_{q^{n}}$. In fact, the number of linearized polynomials in $\mathscr{L}_{n}\left(\mathbb{F}_{q^{n}}\right)$ inducing such idempotent maps is given by

$$
\sum_{k=0}^{n} \frac{|\mathrm{GL}(n, q)|}{|\mathrm{GL}(k, q)||\mathrm{GL}(n-k, q)|}
$$

where $\operatorname{GL}(k, q)$ denotes the group of non-singular $k \times k$ matrices over $\mathbb{F}_{q}$ with (wellknown) order given by $|\operatorname{GL}(k, q)|=\prod_{i=0}^{k-1}\left(q^{k}-q^{i}\right)$ for $k \geq 1$, and $|\operatorname{GL}(0, q)|=1$ by convention. Indeed, we know that internal direct-sum decompositions of $\mathbb{F}_{q^{n}}$, into ordered pairs of trivially-intersected $\mathbb{F}_{q}$-subspaces, are in correspondence with idempotent $\mathbb{F}_{q}$-linear operators of $\mathbb{F}_{q^{n}}$, which in turn correspond to elements in $\mathscr{L}_{n}\left(\mathbb{F}_{q^{n}}\right)$ inducing such idempotent maps. Fixing an integer $k$, where $0 \leq k \leq n$, we note that the bases of $\mathbb{F}_{q^{n}}$ over $\mathbb{F}_{q}$ are in bijective correspondence with ordered pairs of disjoint bases with dimensions $k$ and $n-k$, respectively, over $\mathbb{F}_{q}$. But to each $\mathbb{F}_{q^{-}}$ subspace of $\mathbb{F}_{q^{n}}$ of dimension, say $l$, corresponds $|\mathrm{GL}(l, q)|$ bases of it. Then there are $\frac{|\mathrm{GL}(n, q)|}{|\mathrm{GL}(k, q)||\mathrm{GL}(n-k, q)|}$ decompositions of $\mathbb{F}_{q^{n}}$ into ordered pairs of triviallyintersected $\mathbb{F}_{q}$-subspaces with dimensions $k$ and $n-k$, respectively. The claim now follows. For example, when $n=2$ and $q=2,3,4,5$, this number is 8, 14,22,32, respectively.

We now place our attention to other preliminary lemmata which will be of use in the following sections. The following lemma is a generalization of Lemma 3.1 in [81] and thus serves in tackling more general classes of permutations of arbitrary finite fields. 
Lemma 2.1.10. Let $q=p^{m}$ be a prime power, let $\psi, \bar{\psi} \in \mathbb{F}_{q^{n}}[x]$ be additive, and let $f \in \mathbb{F}_{q^{n}}[x]$. Define the map $\phi_{\psi}: \mathbb{F}_{q^{n}} \rightarrow \psi\left(\mathbb{F}_{q^{n}}\right) \oplus S_{\psi}$ by $\phi_{\psi}(x)=(\psi(x), x-\psi(x))$. Then $\phi_{\psi}$ is injective for any such $\psi$, and, for any $(y, z) \in \phi_{\psi}\left(\mathbb{F}_{q^{n}}\right), \phi_{\psi}^{-1}(y, z)=y+z$. Then $f$ permutes $\mathbb{F}_{q^{n}}$ if and only if the map $F: \phi_{\psi}\left(\mathbb{F}_{q^{n}}\right) \rightarrow \phi_{\bar{\psi}}\left(\mathbb{F}_{q^{n}}\right)$, given by $F=\phi_{\bar{\psi}} \circ f \circ \phi_{\psi}^{-1}$, is bijective. In this case, $F^{-1}=\phi_{\psi} \circ f^{-1} \circ \phi_{\bar{\psi}}^{-1}$.

Proof. It is easy to check that $\phi_{\psi}$ is well-defined and injective for any such $\psi$. Then $\left|\phi_{\psi}\left(\mathbb{F}_{q^{n}}\right)\right|=\left|\phi_{\bar{\psi}}\left(\mathbb{F}_{q^{n}}\right)\right|=\left|\mathbb{F}_{q^{n}}\right|$. Hence, since $F \circ \phi_{\psi}=\phi_{\bar{\psi}} \circ f$, it follows that $f$ is a permutation if and only if $F$ is a bijection (injectivity implies bijectivity since the respective domain and codomain of $f, F$, are equally sized and finite).

This lemma proves valuable since it provides a way of computing the inverses of certain classes of polynomials by computing the inverses of two other polynomials, one of these linearized, on subspaces; this should be easier.

First it would be of interest to determine when is a linear map $\varphi$ a bijection between two similar subspaces $S_{\psi}, S_{\bar{\psi}}$. Under certain restrictions on $\varphi, \psi, \bar{\psi}$, Lemma 2.1.13 shows when this happens. Now we make use of the following lemma which is a slight (more general) variation of the AGW Criterion given in Lemma 1.2, [3].

Lemma 2.1.11. Let $A, \bar{A}, S, \bar{S}$, be finite sets with $|A|=|\bar{A}|$ and $|S|=|\bar{S}|$, and let $f: A \rightarrow \bar{A}, \bar{f}: S \rightarrow \bar{S}, \lambda: A \rightarrow S$, and $\bar{\lambda}: \bar{A} \rightarrow \bar{S}$ be maps such that $\bar{\lambda} \circ f=\bar{f} \circ \lambda$. If both $\lambda$ and $\bar{\lambda}$ are surjective, then the following two statements are equivalent:

(i) $f$ is a bijection from $A$ to $\bar{A}$; and

(ii) $\bar{f}$ is a bijection from $S$ to $\bar{S}$ and $f$ is injective on $\lambda^{-1}(s)$ for each $s \in S$.

Furthermore, if $A, \bar{A}, S, \bar{S}$ form groups under some operation + (although the groups may not be abelian) and $f, \bar{f}, \lambda, \bar{\lambda}$ are homomorphisms on the respective groups, then $f$ is injective on $\lambda^{-1}(s)$ for each $s \in S$ if and only if $\operatorname{ker}(f) \cap \operatorname{ker}(\lambda)=\{0\}$.

Proof. The proof of (i), (ii), is identical to the proof of Lemma 1.2 in [3] and we omit 
it. Now for the group case, assume $\alpha, \beta \in \lambda^{-1}(s):=\{a \in A \mid \lambda(a)=s\}$ such that $\alpha \neq \beta$. Then $0 \neq f(\alpha)-f(\beta)=f(\alpha-\beta)$ if and only if $\operatorname{ker}(f) \cap \operatorname{ker}(\lambda)=\{0\}$ (since $\alpha-\beta \in \operatorname{ker}(\lambda))$ as required.

The following easy consequence gives us a criterion for determining when a linearized polynomial induces a permutation of the finite field in question. We will often apply this useful result in the following sections.

Lemma 2.1.12. Let $\varphi, \psi, \bar{\psi} \in \mathbb{F}_{q^{n}}[x]$ be additive such that $\varphi \circ \psi=\bar{\psi} \circ \varphi$ and $\left|\psi\left(\mathbb{F}_{q^{n}}\right)\right|=$ $\left|\bar{\psi}\left(\mathbb{F}_{q^{n}}\right)\right|$. Then $\varphi$ induces a permutation of $\mathbb{F}_{q^{n}}$ if and only if $\operatorname{ker}(\varphi) \cap \operatorname{ker}(\psi)=$ $\operatorname{ker}(\varphi) \cap \psi\left(\mathbb{F}_{q^{n}}\right)=\{0\}$.

Lemma 2.1.13. Let $\varphi, \psi, \bar{\psi} \in \mathbb{F}_{q^{n}}[x]$ be additive such that $\varphi \circ \psi=\bar{\psi} \circ \varphi,\left|\psi\left(\mathbb{F}_{q^{n}}\right)\right|=$ $\left|\bar{\psi}\left(\mathbb{F}_{q^{n}}\right)\right|$, and $\left|S_{\psi}\right|=\left|S_{\bar{\psi}}\right|$. Then $\varphi$ induces a bijection between $S_{\psi}$ and $S_{\bar{\psi}}$ if and only if $\operatorname{ker}(\varphi) \cap \operatorname{ker}(\psi)=\operatorname{ker}(\varphi) \cap \psi\left(S_{\psi}\right)=\{0\}$.

Proof. First note that because $\left|\psi\left(\mathbb{F}_{q^{n}}\right)\right|=\left|\bar{\psi}\left(\mathbb{F}_{q^{n}}\right)\right|$, then $|\operatorname{ker}(\psi)|=|\operatorname{ker}(\bar{\psi})|$. As $\operatorname{ker}(\psi) \subseteq S_{\psi}, \operatorname{ker}(\bar{\psi}) \subseteq S_{\bar{\psi}}$, and $\left|S_{\psi}\right|=\left|S_{\bar{\psi}}\right|$, it follows that $\left|\psi\left(S_{\psi}\right)\right|=\left|\bar{\psi}\left(S_{\bar{\psi}}\right)\right|$. Moreover, since $\psi\left(S_{\psi}\right) \subseteq S_{\psi}$ and $\varphi \circ \psi=\bar{\psi} \circ \varphi$, we deduce that $\varphi\left(S_{\psi}\right) \subseteq S_{\bar{\psi}}$ and $\varphi\left(\psi\left(S_{\psi}\right)\right) \subseteq \bar{\psi}\left(S_{\bar{\psi}}\right)$. Now Lemma 2.1.11 finishes the proof if we let $A=S_{\psi}, \bar{A}=S_{\bar{\psi}}$, $S=\psi\left(S_{\psi}\right), \bar{S}=\bar{\psi}\left(S_{\bar{\psi}}\right), f=\bar{f}=\varphi, \lambda=\psi$, and $\bar{\lambda}=\bar{\psi}$.

\subsection{DFT for finite fields, period of functions and cyclotomic polynomials}

We recall some preliminary concepts regarding the DFT for finite fields, convolution, least period of functions on cyclic groups, and cyclotomic polynomials ${ }^{2}$.

\footnotetext{
${ }^{2}$ This section is substantively based on some of the content of the following article first published in Canadian Journal of Mathematics at https://doi.org/10.4153/CJM-2017-022-1, Copyright
} 
Let $N \in \mathbb{N}$ such that $N \mid q-1$, and let $\zeta_{N}$ be a primitive $N$-th root of unity in $\mathbb{F}_{q}^{*}$ (the condition on $N$ guarantees the existence of $\zeta_{N}$ ). We shall use the common notation $\mathbb{Z}_{N}:=\mathbb{Z} / N \mathbb{Z}$. Now the DFT based on $\zeta_{N}$, on the $\mathbb{F}_{q}$-vector space of functions $f: \mathbb{Z}_{N} \rightarrow \mathbb{F}_{q}$, is defined by

$$
\mathcal{F}_{\zeta_{N}}[f](i)=\sum_{j \in \mathbb{Z}_{N}} f(j) \zeta_{N}^{i j}, \quad i \in \mathbb{Z}_{N}
$$

Note $\mathcal{F}_{\zeta_{N}}$ is a bijective linear operator with inverse given by $\mathcal{F}_{\zeta_{N}}^{-1}=N^{-1} \mathcal{F}_{\zeta_{N}^{-1}}$.

For $f, g: \mathbb{Z}_{N} \rightarrow \mathbb{F}_{q}$, the convolution of $f, g$ is the function $f \otimes g: \mathbb{Z}_{N} \rightarrow \mathbb{F}_{q}$ given by

$$
(f \otimes g)(i)=\sum_{\substack{j+k=i \\ j, k \in \mathbb{Z}_{N}}} f(j) g(k) .
$$

Inductively, $f_{1} \otimes f_{2} \otimes \cdots \otimes f_{k}=f_{1} \otimes\left(f_{2} \otimes \cdots \otimes f_{k}\right)$ and so

$$
\left(f_{1} \otimes \cdots \otimes f_{k}\right)(i)=\sum_{\substack{j_{1}+\cdots+j_{k}=i \\ j_{1}, \ldots, j_{k} \in \mathbb{Z}_{N}}} f_{1}\left(j_{1}\right) \cdots f_{k}\left(j_{k}\right)
$$

For $m \in \mathbb{N}$, we let $f^{\otimes m}$ denote the $m$-th convolution power of $f$, that is, the convolution of $f$ with itself, $m$ times. The DFT and convolution are related by the fact that

$$
\prod_{i=1}^{k} \mathcal{F}_{\zeta_{N}}\left[f_{i}\right]=\mathcal{F}_{\zeta_{N}}\left[\bigotimes_{i=1}^{k} f_{i}\right]
$$

Since $f, \mathcal{F}_{\zeta_{N}}[f]$, have values in $\mathbb{F}_{q}$ by definition, it follows from the relation above that $f^{\otimes q}=f$. Convolution is associative, commutative and distributive with identity $\delta_{0}: \mathbb{Z}_{N} \rightarrow\{0,1\} \subseteq \mathbb{F}_{p}$, the Kronecker delta function defined by $\delta_{0}(i)=1$ if $i=0$ and (C) 2018, Canadian Mathematical Society. It is released under a Creative Commons Attribution Non-Commercial No Derivatives License.

[72] A. Tuxanidy and Q. Wang, A new proof of the Hansen-Mullen irreducibility conjecture, Canadian Journal of Mathematics, no.70 (2018), 1373-1389. https://doi.org/10.4153/ CJM-2017-022-1 
$\delta_{0}(i)=0$ otherwise. We set $f^{\otimes 0}=\delta_{0}$.

Next we recall the concepts of a period and least period of a function $f: \mathbb{Z}_{N} \rightarrow \mathbb{F}_{q}$. For $r \in \mathbb{N}$, we say that $f$ is $r$-periodic if $f(i)=f(i+\bar{r})$ for all $i \in \mathbb{Z}_{N}$. Clearly $f$ is $r$-periodic if and only if it is $\operatorname{gcd}(r, N)$-periodic. The smallest such positive integer $r$ is called the least period of $f$. Note the least period $r$ satisfies $r \mid R$ whenever $f$ is $R$-periodic. If the least period of $f$ is $N$, we say that $f$ has maximum least period.

There are various operations on cyclic (i.e., periodic) functions which preserve the least period. For instance the $k$-shift function $f_{k}(i):=f(i+k)$ of $f$ has the same least period as $f$. The reversal function $f^{*}(i):=f(-(1+i))$ of $f$ also has the same least period. Let $\sigma$ be a permutation of $\mathbb{F}_{q}$. The function $f^{\sigma}(i):=\sigma(f(i))$ keeps the least period of $f$ as well.

Next we recall a few elementary facts about cyclotomic polynomials. For $n \in \mathbb{N}$, the $n$-th cyclotomic polynomial $\Phi_{n}(x) \in \mathbb{Z}[x]$ is defined by

$$
\Phi_{n}(x)=\prod_{k \in(\mathbb{Z} / n \mathbb{Z})^{\times}}\left(x-\zeta_{n}^{k}\right)
$$

where $\zeta_{n} \in \mathbb{C}$ is a primitive $n$-th root of unity and $(\mathbb{Z} / n \mathbb{Z})^{\times}$denotes the unit group modulo $n$. Since $x^{n}-1=\prod_{d \mid n} \Phi_{d}(x)$, the Möbius inversion formula gives $\Phi_{n}(x)=$ $\prod_{d \mid n}\left(x^{n / d}-1\right)^{\mu(d)}$, where $\mu$ is the Möbius function.

For any divisor $m$ of $n$, with $0<m<n$, we note that

$$
\frac{x^{n}-1}{x^{m}-1}=\frac{\prod_{d \mid n} \Phi_{d}(x)}{\prod_{d \mid m} \Phi_{d}(x)}=\prod_{\substack{d \mid n \\ d \nmid m}} \Phi_{d}(x) .
$$

Hence

$$
\Phi_{n}(x) \mid \frac{x^{n}-1}{x^{m}-1} \in \mathbb{Z}[x] .
$$


In fact, one can show that for $n \geq 2$,

$$
\Phi_{n}(q)=\operatorname{gcd}\left\{\frac{q^{n}-1}{q^{d}-1}: 1 \leq d<n, d \mid n\right\}
$$

and so

$$
\frac{q^{n}-1}{\Phi_{n}(q)}=\operatorname{lcm}\left\{q^{d}-1: 1 \leq d<n, d \mid n\right\}
$$

Note also that

$$
\begin{aligned}
\Phi_{n}(q) & =\left|\Phi_{n}(q)\right|=\prod_{k \in(\mathbb{Z} / n \mathbb{Z})^{\times}}\left|q-\zeta_{n}^{k}\right| \\
& >q-1
\end{aligned}
$$

for $n \geq 2$, since $\left|q-\zeta_{n}^{k}\right|>q-1$ for any primitive $n$-th root $\zeta_{n}^{k} \in \mathbb{C}$, whenever $n \geq 2$ (as can be seen geometrically by looking at the complex plane) ${ }^{3}$.

\subsection{Gaussian sums and periods}

In this section ${ }^{4}$ we collect some results from literature about Gaussian sums and periods which will be of use later on.

We start off by recalling some concepts and definitions. These are well-known and may be found for example in Chapter 5 of [48] and in [26].

Let $S=\{\alpha \in \mathbb{C}:|\alpha|=1\}$. An additive character on $\mathbb{F}_{q}$ is an (additive)

\footnotetext{
${ }^{3}$ The elementary facts in (2.2.1) and (2.2.2) have some historical significance. For instance, these make an appearance in Witt's classical proof of Wedderburn's theorem that every finite division ring is a field (see Chapter 5 in [1] for example).

${ }^{4}$ This section is substantively based on some of the content of the following article originally published in Journal of Number Theory, https://doi.org/10.1016/j.jnt.2015.09.008. It is released under a Creative Commons Attribution Non-Commercial No Derivatives License.

[69] A. Tuxanidy and Q. Wang, On the number of $N$-free elements with prescribed trace, Journal of Number Theory, v.160 (2016), p.536-565. https://doi.org/10.1016/j.jnt.2015.09.008
} 
homomorphism $\chi: \mathbb{F}_{q} \rightarrow S$. One can show these are exactly the mappings of the form $x \mapsto e^{2 \pi \sqrt{-1} \operatorname{Tr}_{\mathbb{F}_{q} / \mathbb{F}_{p}}(a x) / p}$ for some $a \in \mathbb{F}_{q}$. Here $p$ is the characteristic of $\mathbb{F}_{q}$. In the special case when $a=1$, we call the corresponding additive character canonical.

Let $\chi_{q}, \chi_{q^{m}}$, be the canonical additive characters of $\mathbb{F}_{q}, \mathbb{F}_{q^{m}}$, respectively, defined by $\chi_{q}(x)=e^{2 \pi \sqrt{-1} \operatorname{Tr}_{\mathbb{F}_{q} / \mathbb{F}_{p}}(x) / p}$ for $x \in \mathbb{F}_{q}$, and $\chi_{q^{m}}=\chi_{q} \circ \operatorname{Tr}_{\mathbb{F}_{q} m / \mathbb{F}_{q}}$. By the transitivity of the trace function, $\chi_{q^{m}}(z)=e^{2 \pi \sqrt{-1} \operatorname{Tr}_{\mathbb{F}_{q}^{m} / \mathbb{\mathbb { F } p}}(z) / p}$ for $z \in \mathbb{F}_{q^{m}}$. Denote with $\chi_{a}^{\left(q^{m}\right)}$ the additive character of $\mathbb{F}_{q^{m}}$ corresponding to $a \in \mathbb{F}_{q^{m}}$; that is $\chi_{a}^{\left(q^{m}\right)}(z)=\chi_{q^{m}}(a z)$ for any $z \in \mathbb{F}_{q^{m}}$. Clearly $\chi_{1}^{\left(q^{m}\right)}=\chi_{q^{m}}$. The following orthogonality relation will be of use.

$$
\sum_{a \in \mathbb{F}_{q}} \chi_{q}(a x)= \begin{cases}q & \text { if } x=0 \\ 0 & \text { if } x \in \mathbb{F}_{q}^{*}\end{cases}
$$

We similarly define a multiplicative character on $\mathbb{F}_{q^{m}}$ to be a (multiplicative) homomorphism $\psi: \mathbb{F}_{q^{m}}^{\times} \rightarrow S$, where $\mathbb{F}_{q^{m}}^{\times}$denotes the multiplicative group of $\mathbb{F}_{q^{m}}$. Let $\alpha$ be a primitive element of $\mathbb{F}_{q^{m}}$. Then one can also show that the multiplicative characters on $\mathbb{F}_{q^{m}}$ are exactly the mappings of the form $\alpha^{v} \mapsto e^{2 \pi \sqrt{-1} j v / N}$ for some positive divisor $N$ of $q^{m}-1$ and some integer $j$ coprime to $N$. In this case we say that $\psi$ is of order $N$ and usually highlight this by writing $\psi_{N}$ instead of $\psi$.

The Gaussian sums of order $N$ are given by

$$
G_{q^{m}}\left(\psi_{N}, \chi_{a}^{\left(q^{m}\right)}\right)=\sum_{\beta \in \mathbb{F}_{q^{*}}^{*}} \psi_{N}(\beta) \chi_{q^{m}}(a \beta)
$$

We denote $G_{q^{m}}\left(\psi_{N}\right):=G_{q^{m}}\left(\psi_{N}, \chi_{q^{m}}\right)$. Note that if $a \neq 0$, then $G_{q^{m}}\left(\psi_{N}, \chi_{a}^{\left(q^{m}\right)}\right)=$ $\overline{\psi_{N}(a)} G_{q^{m}}\left(\psi_{N}\right)$ (Theorem 5.12 (i), [48]).

For $N \mid q^{m}-1$ (i.e., $N$ divides $\left.q^{m}-1\right)$ the cyclotomic classes of $\mathbb{F}_{q^{m}}^{*}$ of type $\left(N, q^{m}\right)$ are defined by $C_{k}^{\left(N, q^{m}\right)}=\alpha^{k}\left\langle\alpha^{N}\right\rangle$, where $k \in \mathbb{Z}$. Clearly $C_{k}^{\left(N, q^{m}\right)}=C_{0}^{\left(N, q^{m}\right)}$ whenever 
$k \equiv 0(\bmod N)$. Then the Gaussian periods of type $\left(N, q^{m}\right)$ are given by

$$
\eta_{k}^{\left(N, q^{m}\right)}=\sum_{x \in C_{k}^{\left(N, q^{m}\right)}} \chi_{q^{m}}(x) .
$$

In the case when $N=1$, one can easily show that $\eta_{k}^{\left(1, q^{m}\right)}=-1$ for any $k \in \mathbb{Z}$. In the cases when $N \geq 2$, it is however much more difficult to attain explicit formulas for these and only few results are known, for rather small $N$. The Gaussian sums are the discrete Fourier transforms of the Gaussian periods and hence the two are related by the equation

$$
\begin{aligned}
\eta_{k}^{\left(N, q^{m}\right)} & =\frac{1}{N} \sum_{j=0}^{N-1} \sum_{x \in \mathbb{F}_{q}^{*}} \chi_{q^{m}}\left(\alpha^{k} x\right) \psi_{N}^{j}(x)=\frac{1}{N} \sum_{j=0}^{N-1} \overline{\psi_{N}^{j}\left(\alpha^{k}\right)} G_{q^{m}}\left(\psi_{N}^{j}\right) \\
& =\frac{1}{N}\left(-1+\sum_{j=1}^{N-1} \overline{\psi_{N}^{j}\left(\alpha^{k}\right)} G_{q^{m}}\left(\psi_{N}^{j}\right)\right)
\end{aligned}
$$

where $\psi_{N}$ is a multiplicative character of $\mathbb{F}_{q^{m}}$ with order $N$ (see Equation (9) in [26]).

In their study of Hamming weights of irreducible cyclic codes, Ding and Yang [26] recently obtained the following result regarding cyclotomic classes.

Lemma 2.3.1 (Lemma 5, [26]). Let $N$ be a positive divisor of $q^{m}-1$ and let $k \in \mathbb{Z}$. Let $Q:=\left(q^{m}-1\right) /(q-1)$. We have the following multiset equality:

$$
\left\{a x: a \in \mathbb{F}_{q}^{*}, x \in C_{k}^{\left(N, q^{m}\right)}\right\}=\frac{(q-1) \operatorname{gcd}(Q, N)}{N} * C_{k}^{\left(\operatorname{gcd}(Q, N), q^{m}\right)},
$$

where the right hand side denotes the multiset in which each element in the set $C_{k}^{\left(\operatorname{gcd}(Q, N), q^{m}\right)}$ appears in the multiset with multiplicity $\frac{(q-1) \operatorname{gcd}(Q, N)}{N}$.

A consequence to the above is the following. 
Lemma 2.3.2. Let $N \mid q^{m}-1$ and let $k \in \mathbb{Z}$. Then

$$
\sum_{i=0}^{q-2} \eta_{Q i+k}^{\left(N, q^{m}\right)}=\frac{(q-1) \operatorname{gcd}(Q, N)}{N} \eta_{k}^{\left(\operatorname{gcd}(Q, N), q^{m}\right)}
$$

Proof. By the definition of Gaussian periods and by Lemma 2.3.1,

$$
\begin{aligned}
\sum_{i=0}^{q-2} \eta_{Q i+k}^{\left(N, q^{m}\right)} & =\sum_{i=0}^{q-2} \sum_{x \in C_{0}^{\left(N, q^{m}\right)}} \chi_{q^{m}}\left(\alpha^{Q i+k} x\right)=\sum_{i=0}^{q-2} \sum_{x \in C_{k}^{\left(N, q^{m}\right)}} \chi_{q^{m}}\left(\alpha^{Q i} x\right) \\
& =\sum_{a \in \mathbb{F}_{q}^{*}} \sum_{x \in C_{k}^{\left(N, q^{m}\right)}} \chi_{q^{m}}(a x)=\frac{(q-1) \operatorname{gcd}(Q, N)}{N} \sum_{x \in C_{k}^{\left(\operatorname{gcd}(Q, N), q^{m}\right)}} \chi_{q^{m}}(x) \\
& =\frac{(q-1) \operatorname{gcd}(Q, N)}{N} \eta_{k}^{\left(\operatorname{gcd}(Q, N), q^{m}\right)} .
\end{aligned}
$$

Remark 2.3.3. It is known that $\eta_{k}^{\left(N, q^{m}\right)} \in \mathbb{Z}$ whenever $N \mid Q$ (see Theorem 13 (i) in $[26])$.

The following results about Gaussian periods are well known and may be found for example in [26]. We only give the 0-th Gaussian periods as these will be of greater interest to us in the sections that follow. For the other cases we refer the interested reader to $[26]$.

Lemma 2.3.4. When $N=2$, the 0 -th Gaussian periods are given by the following:

$$
\eta_{0}^{\left(2, q^{m}\right)}=\left\{\begin{array}{lll}
\frac{-1+(-1)^{s m-1} q^{m / 2}}{2} & \text { if } p \equiv 1 \quad(\bmod 4) \\
\frac{-1+(-1)^{s m-1}(\sqrt{-1})^{s m} q^{m / 2}}{2} & \text { if } p \equiv 3 \quad(\bmod 4)
\end{array}\right.
$$

In the case when $N=3$ we only give a particular instance although the results in 
other cases are also known.

Lemma 2.3.5. Let $N=3$, let $s m \equiv 0(\bmod 3)$, let $p \equiv 1(\bmod 3)$, and let $(c, d)$ be the unique (up to sign) solutions to the equation $4 p^{s m / 3}=c^{2}+27 d^{2}$ with $c \equiv 1$ $(\bmod 3)$ and $p \nmid c$. Then

$$
\eta_{0}^{\left(3, q^{m}\right)}=\frac{-1+c q^{m / 3}}{3}
$$

The Gaussian periods in the so called semi-primitive case are known as well and are given in the following lemma. See [26].

Lemma 2.3.6. Assume that $N>2$ and there exists a positive integer $j$ such that $p^{j} \equiv-1(\bmod N)$ and that $j$ is the least such. Let $r=p^{2 j \gamma}$ for some integer $\gamma$.

(a) If $\gamma, p$ and $\left(p^{j}+1\right) / N$ are all odd, then

$$
\eta_{0}^{(N, r)}=-\frac{r^{1 / 2}+1}{N}
$$

(b) In all other cases,

$$
\eta_{0}^{(N, r)}=\frac{(-1)^{\gamma+1}(N-1) r^{1 / 2}-1}{N}
$$

The following lemma will be useful as well.

Lemma 2.3.7. Let $q$ be a power of a prime $p$ and let $Q=\left(q^{m}-1\right) /(q-1)$. Then

$$
\eta_{0}^{\left(Q, q^{m}\right)}= \begin{cases}-1 & \text { if } p \nmid m ; \\ q-1 & \text { otherwise. }\end{cases}
$$

Proof. By definition,

$$
\eta_{0}^{\left(Q, q^{m}\right)}=\sum_{x \in\left\langle\alpha^{Q}\right\rangle} \chi_{q^{m}}(x)=\sum_{x \in \mathbb{F}_{q}^{*}} \chi_{q^{m}}(x)=\sum_{x \in \mathbb{F}_{q}^{*}} \chi_{q}\left(\operatorname{Tr}_{\mathbb{F}_{q} m / \mathbb{F}_{q}}(x)\right)=\sum_{x \in \mathbb{F}_{q}^{*}} \chi_{q}(m x)
$$


Now the result follows by (2.3.1). 


\section{Chapter 3}

\section{On the inverses of some classes of permutations of finite fields}

In this chapter ${ }^{1}$ we study the compositional inverses of several classes of permutation polynomials and present several results in this area. We apply the method highlighted in the introduction and proceed to obtain inverses of several classes of permutations. The list of chosen permutations for which we study their inverses is by no means exhaustive, although the method could potentially be used to obtain several more inverses of other classes of permutations not given here. Moreover, more corollaries than we have given here may be possible from our results, which could be obtained by choosing specific instances of the variables $\varphi, \psi, g, h$, etc..

This chapter is organized as follows. In Section 3.1 we prove Theorems 1.0.2, 3.1.10, where we give the compositional inverses of two classes of permutation polynomials given in [3], [88], respectively, written in terms of the inverses of two poly-

\footnotetext{
${ }^{1}$ This chapter is substantively based on the following article originally published in Finite Fields and Their Applications, https://doi.org/10.1016/j.ffa.2014.02.006. It is released under a Creative Commons Attribution Non-Commercial No Derivatives License.

[70] A. Tuxanidy and Q. Wang, On the inverses of some classes of permutations of finite fields, Finite Fields and their Applications, v.28 (2014), p.244-281. https://doi.org/10.1016/j.ffa. 2014.02 .006
} 
nomials permutating subspaces of $\mathbb{F}_{q^{n}}$. We then proceed to obtain several corollaries from them. In Section 3.2 we explicitly obtain, in Theorem 3.2.4 and Corollary 3.2.6, the preimages and compositional inverses, respectively, of a class of permutation polynomials generalizing those in [23] and [81]. Finally in Section 3.3 we prove Theorem 1.0.10, which gives the explicit compositional inverse of a class of permutation polynomials generalizing that of a linearized permutation class whose inverse was recently obtained in [79]. See also Lemma 3.3.3 which corresponds to the case when $G(x)=x$ and $c=1$ below. In particular, the method employed here to obtain such a result, as an application of Theorem 1.0.2, seems considerably less complicated than that of [79].

\subsection{Inverses of some classes of permutations}

Proof of Theorem 1.0.2. First note that since $\left|S_{\psi}\right|=\left|S_{\bar{\psi}}\right|$ and $\operatorname{ker}(\varphi) \cap \psi\left(S_{\psi}\right)=$ $\operatorname{ker}(\varphi) \cap \operatorname{ker}(\psi)=\{0\}$, then $\varphi$ is a bijection from $S_{\psi}$ to $S_{\bar{\psi}}$ (and hence invertible there) by Lemma 2.1.13. Now letting $y:=\psi(x), z:=x-\psi(x)$, we obtain

$$
\begin{aligned}
Y & :=\bar{\psi}(f(x))=h(\psi(x)) \varphi(\psi(x))+\bar{\psi}(g(\psi(x)))=h(y) \varphi(y)+\bar{\psi}(g(y)) \\
& =\bar{f}(y) \in \bar{\psi}\left(\mathbb{F}_{q^{n}}\right) \\
Z & :=f(x)-\bar{\psi}(f(x))=h(y) \varphi(x)+g(y)-h(y) \varphi(y)-\bar{\psi}(g(y)) \\
& =h(y) \varphi(z)+g(y)-\bar{\psi}(g(y)) \in S_{\bar{\psi}} .
\end{aligned}
$$


Then by the definition of $F$ from Lemma 2.1.10, we get

$$
\begin{aligned}
F\left(\phi_{\psi}(x)\right) & =F(y, z)=\phi_{\bar{\psi}}\left(f\left(\phi_{\psi}^{-1}(y, z)\right)\right)=\phi_{\bar{\psi}}(f(y+z)) \\
& =\phi_{\bar{\psi}}(f(x))=(\bar{\psi}(f(x)), f(x)-\bar{\psi}(f(x))) \\
& =(Y, Z) .
\end{aligned}
$$

Hence $F$ is defined by

$$
F(y, z)=(\bar{f}(y), h(y) \varphi(z)+g(y)-\bar{\psi}(g(y)))=(Y, Z)
$$

for $y \in \psi\left(\mathbb{F}_{q^{n}}\right)$ and $z \in S_{\psi}$. Now, from Lemma 2.1 .10 we know that $F$ induces a bijection from $\phi_{\psi}\left(\mathbb{F}_{q^{n}}\right)$ to $\phi_{\bar{\psi}}\left(\mathbb{F}_{q^{n}}\right)$ (because $f$ is a permutation of $\mathbb{F}_{q^{n}}$, by assumption). Since $\varphi$ induces a bijection from $S_{\psi}$ to $S_{\bar{\psi}}$ and $h(y) \neq 0$ for each $y \in \psi\left(\mathbb{F}_{q^{n}}\right)$, it follows that $\bar{\varphi}_{y}(z):=h(y) \varphi(z)+g(y)-\bar{\psi}(g(y))$ is also bijective from $S_{\psi}$ to $S_{\bar{\psi}}$ for each $y \in \psi\left(\mathbb{F}_{q^{n}}\right)$. Moreover, $\bar{f}$ induces a bijection from $\psi\left(\mathbb{F}_{q^{n}}\right)$ to $\bar{\psi}\left(\mathbb{F}_{q^{n}}\right)$ by Theorem 1.0.1 (ii). Thus, since $F(y, z)=\left(\bar{f}(y), \bar{\varphi}_{y}(z)\right)$, we can get the inverse of $F(y, z)$ from the inverses of $\left.\bar{f}(y)\right|_{\psi\left(\mathbb{F}_{q^{n}}\right)}$ and $\left.\bar{\varphi}_{y}(z)\right|_{S_{\psi}}$. Now, because $y=\bar{f}^{-1}(Y)$, we obtain

$$
\begin{aligned}
z & =\left.\varphi^{-1}\right|_{S_{\bar{\psi}}}\left(\frac{Z-g(y)+\bar{\psi}(g(y))}{h(y)}\right) \\
& =\left.\varphi^{-1}\right|_{S_{\bar{\psi}}}\left(\frac{Z-g\left(\bar{f}^{-1}(Y)\right)+\bar{\psi}\left(g\left(\bar{f}^{-1}(Y)\right)\right)}{h\left(\bar{f}^{-1}(Y)\right)}\right) .
\end{aligned}
$$

Hence,

$$
F^{-1}(Y, Z)=\left(\bar{f}^{-1}(Y),\left.\varphi^{-1}\right|_{S_{\bar{\psi}}}\left(\frac{Z-g\left(\bar{f}^{-1}(Y)\right)+\bar{\psi}\left(g\left(\bar{f}^{-1}(Y)\right)\right)}{h\left(\bar{f}^{-1}(Y)\right)}\right)\right) .
$$


As $f^{-1}=\phi_{\psi}^{-1} \circ F^{-1} \circ \phi_{\bar{\psi}}$ by Lemma 2.1.10, then letting $Y=\bar{\psi}(x)$ and $Z=x-\bar{\psi}(x)$, we finally obtain

$$
f^{-1}(x)=\bar{f}^{-1}(\bar{\psi}(x))+\left.\varphi^{-1}\right|_{S_{\bar{\psi}}}\left(\frac{x-\bar{\psi}(x)-g\left(\bar{f}^{-1}(\bar{\psi}(x))\right)+\bar{\psi}\left(g\left(\bar{f}^{-1}(\bar{\psi}(x))\right)\right)}{h\left(\bar{f}^{-1}(\bar{\psi}(x))\right)}\right)
$$

as required.

If $\varphi$ is a bijection from $\psi\left(\mathbb{F}_{q^{n}}\right)$ to $\bar{\psi}\left(\mathbb{F}_{q^{n}}\right)$, then by Lemma 2.1 .12 it permutes $\mathbb{F}_{q^{n}}$ (since additionally $\operatorname{ker}(\varphi) \cap \operatorname{ker}(\psi)=\{0\}$ because $f$ is a permutation). We claim that for any $\bar{y} \in \bar{\psi}\left(\mathbb{F}_{q^{n}}\right), \bar{f}^{-1}(\bar{y})$ satisfies

$$
\bar{f}^{-1}(\bar{y})=\varphi^{-1}\left(\frac{\bar{y}-\bar{\psi}\left(g\left(\bar{f}^{-1}(\bar{y})\right)\right)}{h\left(\bar{f}^{-1}(\bar{y})\right)}\right)
$$

Indeed,

$$
\begin{aligned}
\bar{f}\left(\bar{f}^{-1}(\bar{y})\right) & =h\left(\bar{f}^{-1}(\bar{y})\right) \varphi\left(\bar{f}^{-1}(\bar{y})\right)+\bar{\psi}\left(g\left(\bar{f}^{-1}(\bar{y})\right)\right) \\
& =h\left(\bar{f}^{-1}(\bar{y})\right) \varphi\left(\varphi^{-1}\left(\frac{\bar{y}-\bar{\psi}\left(g\left(\bar{f}^{-1}(\bar{y})\right)\right)}{h\left(\bar{f}^{-1}(\bar{y})\right)}\right)\right)+\bar{\psi}\left(g\left(\bar{f}^{-1}(\bar{y})\right)\right) \\
& =\bar{y}
\end{aligned}
$$

Similarly one can show that $\bar{f}^{-1}(\bar{f}(y))=y$ for each $y \in \psi\left(\mathbb{F}_{q^{n}}\right)$. Now the fact that $\varphi^{-1}$ must be additive yields the result.

We now proceed to obtain several corollaries.

Corollary 3.1.1 (See Theorem 5.5, [3]). Let $a \in \mathbb{F}_{q}$, let $b \in \mathbb{F}_{q^{n}}$, let $P, L \in \mathbb{F}_{q}[x]$ be q-polynomials. Let $H \in \mathbb{F}_{q^{n}}[x]$ such that $H\left(L\left(\mathbb{F}_{q^{n}}\right)\right) \subseteq \mathbb{F}_{q} \backslash\{-a\}$, and assume

$$
f(x)=a P(x)+(P(x)+b) H(L(x))
$$


permutes $\mathbb{F}_{q^{n}}$. Then $\operatorname{ker}(P) \cap \operatorname{ker}(L)=\{0\}$, and if additionally $\operatorname{ker}(P) \cap L\left(S_{L}\right)=\{0\}$, the inverse of $f$ on $\mathbb{F}_{q^{n}}$ is given by

$$
f^{-1}(x)=\bar{f}^{-1}(L(x))+\frac{\left.P^{-1}\right|_{S_{L}}\left(x-L(x)-b H\left(\bar{f}^{-1}(L(x))\right)+L\left(b H\left(\bar{f}^{-1}(L(x))\right)\right)\right)}{a+H\left(\bar{f}^{-1}(L(x))\right)},
$$

where $\bar{f}(x)=(a+H(x)) P(x)+L(b) H(x)$ is a permutation of $L\left(\mathbb{F}_{q^{n}}\right)$.

Proof. This is Theorem 1.0.1 and 1.0.2 with $h=a+H, \varphi=P, \psi=L$, and $g=$ $b H$.

Proof of Corollary 1.0.4. By Theorem 1.0.1, if $f$ is a permutation of $\mathbb{F}_{q^{n}}$, then $\bar{f}(x)=\bar{\psi}(g(x))+h(x) \varphi(x)=h(x) \varphi(x)$ is a bijection from $\psi\left(\mathbb{F}_{q^{n}}\right)$ to $\bar{\psi}\left(\mathbb{F}_{q^{n}}\right)$. In particular, $\left.\bar{f}\right|_{\psi\left(\mathbb{F}_{q^{n}}\right)}$ is surjective. Thus for any $y \in \mathbb{F}_{q^{n}}$, there exists an $x \in \mathbb{F}_{q^{n}}$ such that $\bar{\psi}(y)=h(\psi(x)) \varphi(\psi(x))=\varphi(\psi(x) h(\psi(x)))=\varphi(\psi(x h(\psi(x))))$. Hence $\varphi$ is a surjection from $\psi\left(\mathbb{F}_{q^{n}}\right)$ to $\bar{\psi}\left(\mathbb{F}_{q^{n}}\right)$. But $\left|\psi\left(\mathbb{F}_{q^{n}}\right)\right|=\left|\bar{\psi}\left(\mathbb{F}_{q^{n}}\right)\right|$. Then $\left.\varphi\right|_{\psi\left(\mathbb{F}_{q^{n}}\right)}$ is a bijection from $\psi\left(\mathbb{F}_{q^{n}}\right)$ to $\bar{\psi}\left(\mathbb{F}_{q^{n}}\right)$. In particular, if $h\left(\psi\left(\mathbb{F}_{q^{n}}\right)\right)=c \in \mathbb{F}_{q}^{*}$, then $\bar{f}$ is a bijection from $\psi\left(\mathbb{F}_{q^{n}}\right)$ to $\bar{\psi}\left(\mathbb{F}_{q^{n}}\right)$ if and only if so is $\varphi$. Now, since $\operatorname{ker}(\varphi) \cap \operatorname{ker}(\psi)=\{0\}$ additionally (because $f$ is a permutation), $\varphi$ is a permutation of $\mathbb{F}_{q^{n}}$ by Lemma 2.1.12. Now the rest follows from Theorem 1.0.2 together with the fact that for any $\bar{y} \in \bar{\psi}\left(\mathbb{F}_{q^{n}}\right)$, $\bar{f}^{-1}(\bar{y})=\varphi^{-1}(\bar{y}) / h\left(\bar{f}^{-1}(\bar{y})\right)$.

We denote $Q(x):=x^{q}-x$, and $N(x):=x^{\left(q^{n}-1\right) /(q-1)}$. Since $q$-polynomials over $\mathbb{F}_{q}$ commute under composition, and $T \circ Q=Q \circ T=Q \circ N=0$, the following corollaries are straightforward applications of Corollary 1.0.4.

Corollary 3.1.2 (See Theorem 5.8, [3]). Let $\varphi \in \mathbb{F}_{q}[x]$ be a q-polynomial, let $G \in$ $\mathbb{F}_{q^{n}}[x]$, let $h \in \mathbb{F}_{q^{n}}[x]$ such that $h\left(\mathbb{F}_{q}\right) \subseteq \mathbb{F}_{q} \backslash\{0\}$. Assume that

$$
f(x)=h(T(x)) \varphi(x)+Q(G(T(x)))
$$


is a permutation of $\mathbb{F}_{q^{n}}$. Then $\varphi$ is a permutation of $\mathbb{F}_{q^{n}}$ and the inverse of $f$ on $\mathbb{F}_{q^{n}}$ is given by

$$
f^{-1}(x)=\frac{\varphi^{-1}\left(x-Q \circ G\left(\frac{\varphi^{-1}(T(x))}{h\left(\bar{f}^{-1}(T(x))\right)}\right)\right)}{h\left(\bar{f}^{-1}(T(x))\right)}
$$

where $\bar{f}(x):=\varphi(1) x h(x)$ is a permutation of $\mathbb{F}_{q}$.

Corollary 3.1.3 (See Theorem 5.10, [3]). Let $\varphi \in \mathbb{F}_{q}[x]$ be a q-polynomial, let $G \in$ $\mathbb{F}_{q^{n}}[x]$, let $h \in \mathbb{F}_{q^{n}}[x]$ such that $h\left(Q\left(\mathbb{F}_{q^{n}}\right)\right) \subseteq \mathbb{F}_{q} \backslash\{0\}$. Assume that

$$
f(x)=h(Q(x)) \varphi(x)+T(G(Q(x)))
$$

is a permutation of $\mathbb{F}_{q^{n}}$. Then $\varphi$ is a permutation of $\mathbb{F}_{q^{n}}$ and the inverse of $f$ on $\mathbb{F}_{q^{n}}$ is given by

$$
f^{-1}(x)=\frac{\varphi^{-1}\left(x-T \circ G\left(\frac{\varphi^{-1}(Q(x))}{h\left(\bar{f}^{-1}(Q(x))\right)}\right)\right)}{h\left(\bar{f}^{-1}(Q(x))\right)}
$$

where $\bar{f}(x):=h(x) \varphi(x)$ is a permutation of $Q\left(\mathbb{F}_{q^{n}}\right)$.

As a consequence of Corollary 3.1.3, we get Proposition 1.0.5.

Proof of Proposition 1.0.5. Follows directly from Corollary 3.1 .3 by noticing that $\varphi^{-1}(x)=\left(a x^{q}-b x\right) /\left(a^{2}-b^{2}\right)$ on $\mathbb{F}_{q^{2}}, \varphi^{-1}(Q(x))=Q(x) /(b-a)$, and $T(x)^{q}=T(x)$ for any $x \in \mathbb{F}_{q^{2}}$.

Corollary 3.1.4 (See Theorem 5.10, [3]). Let $\varphi \in \mathbb{F}_{q}[x]$ be a q-polynomial, let $G \in$ $\mathbb{F}_{q^{n}}[x]$, let $h \in \mathbb{F}_{q^{n}}[x]$ such that $h\left(Q\left(\mathbb{F}_{q^{n}}\right)\right) \subseteq \mathbb{F}_{q} \backslash\{0\}$. Let $N(x):=x^{\left(q^{n}-1\right) /(q-1)}$, and assume that

$$
f(x)=h(Q(x)) \varphi(x)+N(G(Q(x)))
$$

is a permutation of $\mathbb{F}_{q^{n}}$. Then $\varphi$ is a permutation of $\mathbb{F}_{q^{n}}$ and the inverse of $f$ on $\mathbb{F}_{q^{n}}$ 
is given by

$$
f^{-1}(x)=\frac{\varphi^{-1}\left(x-N \circ G\left(\frac{\varphi^{-1}(Q(x))}{h\left(\bar{f}^{-1}(Q(x))\right)}\right)\right)}{h\left(\bar{f}^{-1}(Q(x))\right)}
$$

where $\bar{f}(x):=h(x) \varphi(x)$ is a permutation of $Q\left(\mathbb{F}_{q^{n}}\right)$.

Proof of Corollary 1.0.6. In this case $h=1, g=\gamma G$, and $\psi=T$.

Letting $G(x)=x$ in Corollary 1.0.6, the following is straightforward.

Corollary 3.1.5 (See Corollary 3.5, [88]). Let $\varphi \in \mathbb{F}_{q}[x]$ be a q-polynomial permuting $\mathbb{F}_{q^{n}}$, let $\gamma \in \mathbb{F}_{q^{n}}$, let $c=T(\gamma)$, and assume that

$$
f(x)=\varphi(x)+\gamma T(x)
$$

permutes $\mathbb{F}_{q^{n}}$. Then $c+\varphi(1) \neq 0$ and the inverse of $f$ on $\mathbb{F}_{q^{n}}$ is given by

$$
f^{-1}(x)=\varphi^{-1}\left(x-\frac{\gamma T(x)}{c+\varphi(1)}\right) .
$$

Some other consequences of Theorem 1.0.2 are the following corollaries.

Theorem 3.1.6 (Theorem 5.12 (a), [3]). Let $k \geq 2$ be an even integer, let $L(x)=$ $a x^{q}+b x$, where $a, b \in \mathbb{F}_{q}$ such that $a \neq \pm b$. Then both $L$ and

$$
f(x)=L(x)+Q(x)^{k}
$$

are permutations of $\mathbb{F}_{q^{2}}$.

Corollary 3.1.7. Using the same notations as in Theorem 3.1.6, the inverse of $f$ on $\mathbb{F}_{q^{2}}$ is given by

$$
f^{-1}(x)=\frac{a x^{q}-b x}{a^{2}-b^{2}}-\frac{1}{a+b}\left(\frac{Q(x)}{a-b}\right)^{k} .
$$


Proof. Here $\psi=Q, g(x)=x^{k}, \varphi=L, h=1$, and $\bar{f}(x)=Q\left(x^{k}\right)+L(x)$ is a permutation of $Q\left(\mathbb{F}_{q^{n}}\right)$. The fact that $k \geq 2$ is even gives $Q\left[(Q(x))^{k}\right]=0$ for any $x \in \mathbb{F}_{q^{2}}$. Then $\bar{f}(Q(x))=L(Q(x))=Q(L(x))$; thus $\bar{f}^{-1}(Q(x))=L^{-1}(Q(x))$. Then by Theorem 1.0.2 we obtain

$$
f^{-1}(x)=L^{-1}\left(x-\left(L^{-1}(Q(x))\right)^{k}\right) .
$$

Note that since $a, b \in \mathbb{F}_{q}$ and $a \neq \pm b$, then $a^{2}-b^{2} \in \mathbb{F}_{q}^{*}$. The reader can check that $L^{-1}(x)=\left(a x^{q}-b x\right) /\left(a^{2}-b^{2}\right)$ from which it follows that $L^{-1}(Q(x))=Q(x) /(b-a)$. Then using the fact that $k$ is even we obtain

$$
\begin{aligned}
L^{-1}\left(\left(L^{-1}(Q(x))\right)^{k}\right) & =L^{-1}\left(\left(\frac{Q(x)}{b-a}\right)^{k}\right)=\frac{a\left(\frac{Q(x)}{b-a}\right)^{q k}-b\left(\frac{Q(x)}{b-a}\right)^{k}}{a^{2}-b^{2}} \\
& =\frac{a\left(\frac{Q(x)}{b-a}\right)^{k}-b\left(\frac{Q(x)}{b-a}\right)^{k}}{a^{2}-b^{2}} \\
& =\frac{1}{a+b}\left(\frac{Q(x)}{a-b}\right)^{k},
\end{aligned}
$$

from which the result follows since $L^{-1}$ is additive.

Theorem 1.0.2 also yields a proof of Corollary 1.0.8.

Proof of Corollary 1.0.8. We have $\psi=L_{1}, \varphi=L_{2}$ is a permutation of $\mathbb{F}_{q^{n}}$ and particularly of $L_{1}\left(\mathbb{F}_{q^{n}}\right)$ by Corollary 6.2 in [26], $h=1, g(x)=(G(x))^{s}$. From the proof of Corollary 6.2 in [88], $L_{1}\left[\left(G\left(L_{1}(x)\right)\right)^{s}\right]=0$, and $\bar{f}=L_{2}$ is a permutation of $L_{1}\left(\mathbb{F}_{q^{n}}\right)$. Now Theorem 1.0.2 gives the result.

The following is a direct consequence of Corollary 1.0.8 above and of Corollary 6.2 in [88]. 
Corollary 3.1.8 (See Corollary 6.3, [88]). Let $n$ and $k$ be positive integers such that $\operatorname{gcd}(n, k)=d>1$, and let $s$ be a positive integer with $s\left(q^{k}-1\right) \equiv 0\left(\bmod q^{n}-1\right)$. Then

$$
f(x)=x+\left(x^{q^{k}}-x+\delta\right)^{s}
$$

permutes $\mathbb{F}_{q^{n}}$ for any $\delta \in \mathbb{F}_{q^{n}}$, and the inverse of $f$ on $\mathbb{F}_{q^{n}}$ is given by

$$
f^{-1}(x)=x-\left(x^{q^{k}}-x+\delta\right)^{s}
$$

Proof. This is Corollary 1.0.8 with $L_{1}(x)=x^{q^{k}}-x, G(x)=x+\delta, L_{2}(x)=x$. Since $L_{2}^{-1}(x)=x$, the result follows.

Next in Theorem 3.1.10 we give an expression for the compositional inverse of the following class of permutation polynomials obtained in [88], given in terms of certain inverses of bijections of subspaces.

Theorem 3.1.9 (Theorem 3.1, [88]). Let $r \geq 1$ and $n \geq 1$ be positive integers. Let $\psi, L_{1}, \ldots, L_{r} \in \mathbb{F}_{q}[x]$ be q-polynomials, $g \in \mathbb{F}_{q^{n}}[x], h_{1}, \ldots, h_{r} \in \mathbb{F}_{q}[x]$ and $\delta_{1}, \ldots, \delta_{r} \in$ $\mathbb{F}_{q^{n}}$ such that $\psi\left(\delta_{i}\right) \in \mathbb{F}_{q}$ and $h_{i}\left(\psi\left(\mathbb{F}_{q^{n}}\right)\right) \subseteq \mathbb{F}_{q}$. Then

$$
f(x)=g(\psi(x))+\sum_{i=1}^{r}\left(L_{i}(x)+\delta_{i}\right) h_{i}(\psi(x))
$$

is a permutation polynomial of $\mathbb{F}_{q^{n}}$ if and only if the following two conditions hold.

(i) $\bar{f}(x):=\psi(g(x))+\sum_{i=1}^{r}\left(L_{i}(x)+\psi\left(\delta_{i}\right)\right) h_{i}(x)$ permutes $\psi\left(\mathbb{F}_{q^{n}}\right)$; and

(ii) for any $y \in \psi\left(\mathbb{F}_{q^{n}}\right), \varphi_{y}(x):=\sum_{i=1}^{r} L_{i}(x) h_{i}(y)$ permutes $\operatorname{ker}(\psi)$.

Theorem 3.1.10. Using the same notations as in Theorem 3.1.9, assume $f$ permutes $\mathbb{F}_{q^{n}}$, and let $\bar{f}^{-1}$ be the inverse of $\left.\bar{f}\right|_{\psi\left(\mathbb{F}_{q^{n}}\right)}$. Then if $x \in \mathbb{F}_{q^{n}}$ is such that $\operatorname{ker}\left(\varphi_{\bar{f}^{-1}(\psi(x))}\right) \cap$ 
$\psi\left(S_{\psi}\right)=\{0\}$, the preimage of $x$ under $f$ is given by

$$
\begin{array}{r}
f^{-1}(x)=\bar{f}^{-1}(\psi(x))+\left.\varphi_{\bar{f}^{-1}(\psi(x))}^{-1}\right|_{S_{\psi}}(x \\
-\psi(x)-g\left(\bar{f}^{-1}(\psi(x))\right)-\sum_{i=1}^{r} \delta_{i} h_{i}\left(\bar{f}^{-1}(\psi(x))\right) \\
\left.+\psi\left(g\left(\bar{f}^{-1}(\psi(x))\right)+\sum_{i=1}^{r} \delta_{i} h_{i}\left(\bar{f}^{-1}(\psi(x))\right)\right)\right) .
\end{array}
$$

Furthermore, if $x \in \mathbb{F}_{q^{n}}$ is such that $\operatorname{ker}\left(\varphi_{\bar{f}^{-1}(\psi(x))}\right) \cap \psi\left(\mathbb{F}_{q^{n}}\right)=\{0\}$, then $\varphi_{\bar{f}^{-1}(\psi(x))}$ permutes $\mathbb{F}_{q^{n}}$, and the preimage of $x$ under $f$ is given by

$$
f^{-1}(x)=\varphi_{\bar{f}^{-1}(\psi(x))}^{-1}\left(x-g\left(\bar{f}^{-1}(\psi(x))\right)-\sum_{i=1}^{r} \delta_{i} h_{i}\left(\bar{f}^{-1}(\psi(x))\right)\right)
$$

Proof. As each $L_{i}$ is $q$-linear over $\mathbb{F}_{q}$ and each $h_{i}(y) \in \mathbb{F}_{q}$ for any $y \in \psi\left(\mathbb{F}_{q^{n}}\right)$, then $\varphi_{y}$ is also $q$-linear over $\mathbb{F}_{q}$ for any such $y$. Then $\varphi_{y} \circ \psi=\psi \circ \varphi_{y}$. Of course, $L_{i} \circ \psi=\psi \circ L_{i}$ as well. Thus we apply Lemma 2.1.10 with $F=\phi_{\psi} \circ f \circ \phi_{\psi}^{-1}$. As before we let $y:=\psi(x)$ and $z:=x-\psi(x)$. We have

$$
\begin{aligned}
Y & :=\psi(f(x))=\psi(g(y))+\sum_{i=1}^{r}\left(L_{i}(y)+\psi\left(\delta_{i}\right)\right) h_{i}(y)=\bar{f}(y) ; \text { and } \\
Z & :=f(x)-\psi(f(x))=g(y)-\psi(g(y))+\sum_{i=1}^{r}\left(L_{i}(z)+\delta_{i}-\psi\left(\delta_{i}\right)\right) h_{i}(y) \\
& =\varphi_{y}(z)+g(y)+\sum_{i=1}^{r} \delta_{i} h_{i}(y)-\psi\left(g(y)+\sum_{i=1}^{r} \delta_{i} h_{i}(y)\right)
\end{aligned}
$$

Hence $F$ is defined by $F(y, z)=(Y, Z)$. If for $y=\bar{f}^{-1}(Y), \operatorname{ker}\left(\varphi_{y}\right) \cap \psi\left(S_{\psi}\right)=\{0\}$, then $\varphi_{y}$ is invertible on $S_{\psi}$ by Lemma 2.1 .13 since $\operatorname{ker}\left(\varphi_{y}\right) \cap \operatorname{ker}(\psi)=\{0\}$ additionally (because $f$ is a permutation). For such $y$ we obtain

$$
z=\left.\varphi_{y}^{-1}\right|_{S_{\psi}}\left(Z-g(y)-\sum_{i=1}^{r} \delta_{i} h_{i}(y)+\psi\left(g(y)+\sum_{i=1}^{r} \delta_{i} h_{i}(y)\right)\right)
$$


Hence

$$
\begin{aligned}
F^{-1}(Y, Z)=\left(\bar{f}^{-1}(Y),\left.\varphi_{\bar{f}^{-1}(Y)}^{-1}\right|_{S_{\psi}}(Z\right. & -g\left(\bar{f}^{-1}(Y)\right)-\sum_{i=1}^{r} \delta_{i} h_{i}\left(\bar{f}^{-1}(Y)\right) \\
& \left.\left.+\psi\left(g\left(\bar{f}^{-1}(Y)\right)+\sum_{i=1}^{r} \delta_{i} h_{i}\left(\bar{f}^{-1}(Y)\right)\right)\right)\right) .
\end{aligned}
$$

Since $f^{-1}=\phi_{\psi}^{-1} \circ F^{-1} \circ \phi_{\psi}$, letting $Y=\psi(x)$ and $Z=x-\psi(x)$, we obtain

$$
\begin{array}{r}
f^{-1}(x)=\bar{f}^{-1}(\psi(x))+\left.\varphi_{\bar{f}^{-1}(\psi(x))}^{-1}\right|_{S_{\psi}}(x \\
-\psi(x)-g\left(\bar{f}^{-1}(\psi(x))\right)-\sum_{i=1}^{r} \delta_{i} h_{i}\left(\bar{f}^{-1}(\psi(x))\right) \\
\left.+\psi\left(g\left(\bar{f}^{-1}(\psi(x))\right)+\sum_{i=1}^{r} \delta_{i} h_{i}\left(\bar{f}^{-1}(\psi(x))\right)\right)\right)
\end{array}
$$

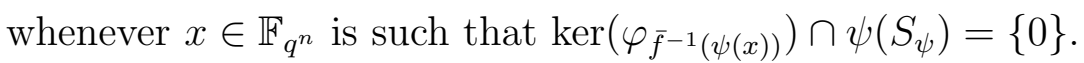

If $y \in \psi\left(\mathbb{F}_{q^{n}}\right)$ is such that $\varphi_{\bar{f}^{-1}(y)}$ permutes $\psi\left(\mathbb{F}_{q^{n}}\right)$, then $\varphi_{\bar{f}^{-1}(y)}$ permutes $\mathbb{F}_{q^{n}}$ by Lemma 2.1.12. We claim that $\bar{f}^{-1}(y)$ satisfies

$$
\bar{f}^{-1}(y)=\varphi_{\bar{f}^{-1}(y)}^{-1}\left(y-\psi\left(g\left(\bar{f}^{-1}(y)\right)+\sum_{i=1}^{r} \delta_{i} h_{i}\left(\bar{f}^{-1}(y)\right)\right)\right) .
$$

Indeed,

$$
\begin{aligned}
\bar{f}^{-1}(\bar{f}(y)) & =\varphi_{y}^{-1}\left(\bar{f}(y)-\psi\left(g(y)+\sum_{i=1}^{r} \delta_{i} h_{i}(y)\right)\right) \\
& =\varphi_{y}^{-1}\left(\psi(g(y))+\sum_{i=1}^{r}\left(L_{i}(y)+\psi\left(\delta_{i}\right)\right) h_{i}(y)-\psi\left(g(y)+\sum_{i=1}^{r} \delta_{i} h_{i}(y)\right)\right) \\
& =\varphi_{y}^{-1}\left(\sum_{i=1}^{r} L_{i}(y) h_{i}(y)\right)=\varphi_{y}^{-1}\left(\varphi_{y}(y)\right) \\
& =y .
\end{aligned}
$$

Similarly one can show that $\bar{f}\left(\bar{f}^{-1}(y)\right)=y$. Now using the fact that $\varphi_{\bar{f}^{-1}(y)}^{-1}$ is 
additive, we get the result.

In particular in the case when $\psi=T$, Theorem 3.1 .10 yields the following.

Corollary 3.1.11 (See Corollary 3.3, [88]). Let $r \geq 1$ and $n \geq 1$ be positive integers. Let $L_{1}, \ldots, L_{r} \in \mathbb{F}_{q}[x]$ be q-polynomials, $g \in \mathbb{F}_{q^{n}}[x], h_{1}, \ldots, h_{r} \in \mathbb{F}_{q}[x]$, and $\delta_{1}, \ldots, \delta_{r} \in \mathbb{F}_{q^{n}}$. If

$$
f(x)=g(T(x))+\sum_{i=1}^{r}\left(L_{i}(x)+\delta_{i}\right) h_{i}(T(x))
$$

is a permutation of $\mathbb{F}_{q^{n}}$, then $\bar{f}(x):=T(g(x))+\sum_{i=1}^{r}\left(L_{i}(1) x+T\left(\delta_{i}\right)\right) h_{i}(x)$ is a permutation of $\mathbb{F}_{q}$, and $\varphi_{y}(x):=\sum_{i=1}^{r} L_{i}(x) h_{i}(y)$ is a permutation of $\operatorname{ker}(T)$ for any $y \in \mathbb{F}_{q}$.

(i) If $p \mid n$ or $x \in \mathbb{F}_{q^{n}}$ is such that $\sum_{i=1}^{r} L_{i}(1) h_{i}\left(\bar{f}^{-1}(T(x))\right) \neq 0$ (equivalently, $\varphi_{\bar{f}^{-1}(T(x))}$ permutes $\left.\mathbb{F}_{q}\right)$, then $\varphi_{\bar{f}^{-1}(T(x))}$ permutes $\mathbb{F}_{q^{n}}$, and the preimage of $x$ under $f$ is given by

$$
f^{-1}(x)=\varphi_{\bar{f}^{-1}(T(x))}^{-1}\left(x-g\left(\bar{f}^{-1}(T(x))\right)-\sum_{i=1}^{r} \delta_{i} h_{i}\left(\bar{f}^{-1}(T(x))\right)\right) .
$$

(ii) If $p \nmid n$, the inverse of $f$ on $\mathbb{F}_{q^{n}}$ is given by

$$
\begin{aligned}
f^{-1}(x)=n^{-1} \bar{f}^{-1}(T(x))+ & \\
+\varphi_{\bar{f}^{-1}(T(x))}^{-1} \mid \operatorname{ker}(T) & \left(x-n^{-1} T(x)-g\left(\bar{f}^{-1}(T(x))\right)-\sum_{i=1}^{r} \delta_{i} h_{i}\left(\bar{f}^{-1}(T(x))\right)\right. \\
& \left.+n^{-1} T\left(g\left(\bar{f}^{-1}(T(x))\right)+\sum_{i=1}^{r} \delta_{i} h_{i}\left(\bar{f}^{-1}(T(x))\right)\right)\right) .
\end{aligned}
$$

Proof. That $\bar{f}, \varphi_{y}$, are permutations of $\mathbb{F}_{q}$ and $\operatorname{ker}(T)$, respectively, follows from Theorem 3.1.9. 
(i) If $p \mid n$, then $\mathbb{F}_{q} \subseteq \operatorname{ker}(T)$ since $T(c)=n c=0$ for any $c \in \mathbb{F}_{q}$. But since $\varphi_{y}$ permutes $\operatorname{ker}(T)$ for any $y \in \mathbb{F}_{q}$, and $\varphi_{y}\left(\mathbb{F}_{q}\right) \subseteq \mathbb{F}_{q}$, it follows that $\varphi_{y}$ permutes $\mathbb{F}_{q}$. Now Lemma 2.1.12 implies $\varphi_{y}$ permutes $\mathbb{F}_{q^{n}}$ for any $y \in \mathbb{F}_{q}$. That $\varphi_{y}$ permuting $\mathbb{F}_{q}$ is equivalent with $\sum_{i=1}^{r} L_{i}(1) h_{i}(y) \neq 0$ follows from the fact that each $L_{i}$ is a $q$-polynomial over $\mathbb{F}_{q}$. The rest follows from Theorem 3.1.10.

(ii) If $p \nmid n$, then $\operatorname{ker}(T)=\left\{x-n^{-1} T(x) \mid x \in \mathbb{F}_{q^{n}}\right\}$; thus if we let $\psi=n^{-1} T$, we get $S_{\psi}=\operatorname{ker}(\psi)=\operatorname{ker}(T)$. Note that if we define the polynomial $H(x):=$ $f\left(n^{-1} x\right)$, then we obtain an instance of Theorem 3.1.9 with $\psi=n^{-1} T, \bar{H}=n^{-1} \bar{f}$ and $\varphi_{y}^{(H)}=n^{-1} \varphi_{y}$, from which it follows that $\bar{H}^{-1}\left(n^{-1} T(x)\right)=\bar{f}^{-1}(T(x))$, and $\left.\varphi_{y}^{-1(H)}\right|_{\operatorname{ker}(T)}=\left.n \varphi_{y}^{-1}\right|_{\operatorname{ker}(T)}$. Now since $f(x)=H(n x)$, then $f^{-1}(x)=n^{-1} H^{-1}(x)$, and the result follows from Theorem 3.1.10.

We conclude with a few more corollaries. The first one below is an application of Theorem 3.1.10.

Corollary 3.1.12 (See Corollary 3.2, [88]). Let $L_{1}, L_{2}, L_{3}: \mathbb{F}_{q^{n}} \rightarrow \mathbb{F}_{q^{n}}$ be q-polynomials over $\mathbb{F}_{q}$. Let $w \in \mathbb{F}_{q^{n}}[x]$ such that $w\left(L_{3}\left(\mathbb{F}_{q^{n}}\right)\right) \subseteq \mathbb{F}_{q}$, and assume that

$$
f(x)=L_{1}(x)+L_{2}(x) w\left(L_{3}(x)\right)
$$

permutes $\mathbb{F}_{q^{n}}$. Let $\bar{f}(x)=L_{1}(x)+L_{2}(x) w(x)$, and for $y \in L_{3}\left(\mathbb{F}_{q^{n}}\right)$, let $\varphi_{y}(x)=$ $L_{1}(x)+L_{2}(x) w(y)$. Then if $x \in \mathbb{F}_{q^{n}}$ is such that $\operatorname{ker}\left(\varphi_{\bar{f}^{-1}\left(L_{3}(x)\right)}\right) \cap L_{3}\left(S_{L_{3}}\right)=\{0\}$, the preimage of $x$ under $f$ is given by

$$
f^{-1}(x)=\bar{f}^{-1}\left(L_{3}(x)\right)+\left.\varphi_{\bar{f}^{-1}\left(L_{3}(x)\right)}^{-1}\right|_{S_{L_{3}}}\left(x-L_{3}(x)\right) .
$$

Furthermore, if $x \in \mathbb{F}_{q^{n}}$ is such that $\varphi_{\bar{f}^{-1}\left(L_{3}(x)\right)}$ permutes $L_{3}\left(\mathbb{F}_{q^{n}}\right)$, then $\varphi_{\bar{f}^{-1}\left(L_{3}(x)\right)}$ 
permutes $\mathbb{F}_{q^{n}}$, and the preimage of $x$ under $f$ is given by

$$
f^{-1}(x)=\varphi_{\bar{f}^{-1}\left(L_{3}(x)\right)}^{-1}(x) .
$$

Proof. Here $r=2, g=0, h_{1}=1, h_{2}=w, \delta_{1}=\delta_{2}=0$, and $\psi=L_{3}$. In the case of $\varphi_{\bar{f}^{-1}(y)}$ permuting $L_{3}\left(\mathbb{F}_{q^{n}}\right)$ for $y \in L_{3}\left(\mathbb{F}_{q^{n}}\right)$, it permutes $\mathbb{F}_{q^{n}}$ since it additionally permutes $\operatorname{ker}\left(L_{3}\right)$ because $f$ is a permutation. Then since $\bar{f}(y)=\varphi_{y}(y)$, we get $\bar{f}^{-1}(y)=\varphi_{\bar{f}^{-1}(y)}^{-1}(y)$, from which the result follows.

The proof of Theorem 1.0.9 is essentially the same as the proof of Theorem 3.1.12, where the linear equation in part (b) follows from Corollary 2.1.7.

Theorem 3.1.13 (Theorem 3, [24]). Let $q=p^{m}$ be a prime power, let $g \in \mathbb{F}_{q}[x]$, let $H \in \mathbb{F}_{q}[x]$ be additive, and let $f(x)=H(x)+x g(T(x))$. Then $f(x)$ is a permutation polynomial of $\mathbb{F}_{q^{n}}$ if and only if the following two conditions hold.

(i) For any $y \in \mathbb{F}_{q}$ and any $x \in \mathbb{F}_{q^{n}}$ we have $\varphi_{y}(x):=H(x)+x g(y)=0$ and $T(x)=0$ if and only if $x=0$.

(ii) $\bar{f}(x):=H(x)+x g(x)$ is a permutation polynomial of $\mathbb{F}_{q}$.

Corollary 3.1.14. Using the same notations as in Theorem 3.1.13, we have that $\varphi_{y}(x)=H(x)+x g(y)$ is a permutation of $\operatorname{ker}(T)$, for any $y \in \mathbb{F}_{q}$.

(i) If $p \mid n$ or $x \in \mathbb{F}_{q^{n}}$ is such that $\varphi_{\bar{f}^{-1}(T(x))}$ permutes $\mathbb{F}_{q}$, then $\varphi_{\bar{f}^{-1}(T(x))}$ permutes $\mathbb{F}_{q^{n}}$, and the preimage of $x$ under $f$ is given by

$$
f^{-1}(x)=\varphi_{\bar{f}^{-1}(T(x))}^{-1}(x)
$$

(ii) If $p \nmid n$, then the inverse of $f$ on $\mathbb{F}_{q^{n}}$ is given by

$$
f^{-1}(x)=n^{-1} \bar{f}^{-1}(T(x))+\varphi_{\bar{f}^{-1}(T(x))}^{-1} \mid \operatorname{ker}(T)\left(x-n^{-1} T(x)\right) .
$$


Proof. It follows directly from Corollary 3.1.11, 3.1.12, and Theorem 3.1.13.

\subsection{Compositional inverse of a general class}

In this section we give the compositional inverse of a class of permutation polynomials generalizing those considered in [23] and [81], respectively. First in Lemma 3.2.1 we give a compositional inverse over $\operatorname{ker}(T)$ of a simple $p$-polynomial inducing a permutation of $\operatorname{ker}(T)$. Then, using this result, we give, in Theorem 3.2.4 and Corollary 3.2.6, the preimages and compositional inverse, respectively, of a more general class of permutation polynomials. Similarly as done in [81], our result is left as an expression in terms of the inverse of $\bar{f}$ over the subspace $\mathbb{F}_{q}$.

Lemma 3.2.1. Let $q=p^{m}$ be a power of a prime $p$, let $c \in \mathbb{F}_{q}^{*}$, let $P_{c}(x)=x^{p}+c x$, and for $0 \leq j \leq m-1$ let $a_{j}=(-1)^{j} c^{-\left(p^{j+1}-1\right) /(p-1)}$. Then $P_{c}$ permutes $\operatorname{ker}(T)=$ $\left\{\beta^{q}-\beta \mid \beta \in \mathbb{F}_{q^{n}}\right\}$ if and only if $c$ belongs to any of the following two cases, for which a corresponding compositional inverse on $\operatorname{ker}(T)$ is given as follows.

Case 1: $c^{\left(p^{m}-1\right) /(p-1)}=(-1)^{m}$ and $p \nmid n$.

For any $\delta \in \mathbb{F}_{p}$,

$$
P_{c}^{-1}(x)=\frac{1}{n} \sum_{j=0}^{m-1} \sum_{k=0}^{n-1} a_{j}(\delta-k) x^{p^{k m+j}}
$$

Case 2: $c^{n\left(p^{m}-1\right) /(p-1)} \neq(-1)^{m n}$. Equivalently, $P_{c}$ permutes $\mathbb{F}_{q^{n}}$.

The inverse of $P_{c}$ on $\mathbb{F}_{q^{n}}$, and hence on $\operatorname{ker}(T)$, is given by

$$
P_{c}^{-1}(x)=\frac{1}{c^{n\left(p^{m}-1\right) /(p-1)}+(-1)^{m n-1}} \sum_{i=0}^{m n-1}(-1)^{i} c^{\sum_{j=i+1}^{m n-1} p^{j}} x^{p^{i}} .
$$


Proof. Case 1: Assuming that $c^{\left(p^{m}-1\right) /(p-1)}=(-1)^{m}, p \nmid n$, and using the facts that $a_{j} c^{p^{j}}=-a_{j-1}$ for $j>0, a_{m-1}=-1$, and $a_{0}=c^{-1}$, we get

$$
\begin{aligned}
P_{c}^{-1}\left(P_{c}(x)\right) & =n^{-1} \sum_{j=0}^{m-1} \sum_{k=0}^{n-1} a_{j}(\delta-k)\left(x^{p}+c x\right)^{p^{k m+j}} \\
& =n^{-1} \sum_{j=0}^{m-1} \sum_{k=0}^{n-1} a_{j}(\delta-k)\left(x^{p^{k m+j+1}}+c^{p^{j}} x^{p^{k m+j}}\right) \\
& =n^{-1} \sum_{j=1}^{m} \sum_{k=0}^{n-1} a_{j-1}(\delta-k) x^{p^{k m+j}}+n^{-1} \sum_{j=0}^{m-1} \sum_{k=0}^{n-1} a_{j}(\delta-k) c^{p^{j}} x^{p^{k m+j}} \\
& =n^{-1} \sum_{k=0}^{n-1}(k-\delta) x^{p^{(k+1) m}}+n^{-1} \sum_{k=0}^{n-1}(\delta-k) x^{p^{k m}} \\
& =n^{-1} \sum_{k=1}^{n}(k-1-\delta) x^{p^{k m}}+n^{-1} \sum_{k=0}^{n-1}(\delta-k) x^{p^{k m}}(\delta-k)\left(a_{j-1}+a_{j} c^{p^{j}}\right) x^{p^{k m+j}} \\
& =n^{-1}(n-1) x-n^{-1} \sum_{k=1}^{n-1} x^{p^{k m}} \\
& =n^{-1}(n-1) x+n^{-1}(x-T(x)) \\
& x-n^{-1} T(x) \\
& x,
\end{aligned}
$$

for all $x \in \operatorname{ker}(T)$. Similarly, using the fact that $c a_{j}=-a_{j-1}^{p}$ for $j>0$, one can show that $P_{c}\left(P_{c}^{-1}(x)\right)=x$ for all $x \in \operatorname{ker}(T)$. Note that $P_{c}^{-1}$ must be unique upon reduction modulo $T$; we may set for instance $\delta=0$. Moreover, the existence of the inverse $P_{c}^{-1}$ on $\operatorname{ker}(T)$ is equivalent with $P_{c}$ permuting $\operatorname{ker}(T)$ as maps are invertible on a set if and only if they induce bijections of that set.

In the case when $p \mid n$, we show that $P_{c}$ does not permute $\operatorname{ker}(T)$ for any such $c$. Note that $T(k)=n k=0$ for any $k \in \mathbb{F}_{q}$; thus $\mathbb{F}_{q} \subseteq \operatorname{ker}(T)$. Then since $P_{c}\left(\mathbb{F}_{q}\right) \subseteq \mathbb{F}_{q}$ 
additionally, it suffices to show that $P_{c}$ does not permute $\mathbb{F}_{q}$. To do this we show that the determinant of $m \times m$ associate Dickson matrix, $D_{P_{c}}$, is zero. Indeed,

$$
D_{P_{c}}=\left(\begin{array}{cccccc}
c & 1 & 0 & 0 & \cdots & 0 \\
0 & c^{p} & 1 & 0 & \cdots & 0 \\
\vdots & \vdots & & & & \vdots \\
1 & 0 & 0 & 0 & \cdots & c^{p^{m-1}}
\end{array}\right)
$$

thus, applying the cofactor expansion in the first column, we get $\operatorname{det}\left(D_{P_{c}}\right)=c^{\left(p^{m}-1\right) /(p-1)}+$ $(-1)^{m-1}=0$, by assumption. This completes the proof of Case 1 . Note that we have also obtained that $P_{c}$ is a permutation of $\mathbb{F}_{q}$ if and only if $c^{\left(p^{m}-1\right) /(p-1)} \neq(-1)^{m}$.

Case 2: Otherwise assume $c^{\left(p^{m}-1\right) /(p-1)} \neq(-1)^{m}$. We show that $P_{c}$ is a permutation of $\operatorname{ker}(T)$ if and only if it is a permutation of $\mathbb{F}_{q^{n}}$ which happens if and only if $c^{n\left(p^{m}-1\right) /(p-1)} \neq(-1)^{m n}$. Now, from the proof of Case 1 we know that in this case $P_{c}$ is a permutation of $\mathbb{F}_{q}$. Since $p$-polynomials over $\mathbb{F}_{q}$ commute with $q$-polynomials over $\mathbb{F}_{p}$, we have $P_{c} \circ T=T \circ P_{c}$. Thus we can apply Lemma 2.1.12 to obtain that $P_{c}$ is a permutation of $\operatorname{ker}(T)$ if and only if it permutes $\mathbb{F}_{q^{n}}$ (since $T\left(\mathbb{F}_{q^{n}}\right)=\mathbb{F}_{q}$ additionally). Thus, similarly as done in Case 1, we compute the determinant of the $m n \times m n$ associate Dickson matrix. We have

$$
D_{P_{c}}=\left(\begin{array}{cccccc}
c & 1 & 0 & 0 & \cdots & 0 \\
0 & c^{p} & 1 & 0 & \cdots & 0 \\
\vdots & \vdots & & & & \vdots \\
1 & 0 & 0 & 0 & \cdots & c^{p^{m n-1}}
\end{array}\right)
$$

hence, using the fact that $c \in \mathbb{F}_{q}$, we $\operatorname{get} \operatorname{det}\left(D_{P_{c}}\right)=c^{\sum_{i=0}^{m n-1} p^{i}}+(-1)^{m n-1}=$ $c^{n\left(p^{m}-1\right) /(p-1)}+(-1)^{m n-1}$. Therefore $P_{c}$ is a permutation of $\mathbb{F}_{q^{n}}$ if and only if $c^{n\left(p^{m}-1\right) /(p-1)} \neq$ 
$(-1)^{m n}$. Here we will use Proposition 2.1.1 to obtain an expression for $P_{c}^{-1}$. For this we need the $(i, 0)$-th cofactors, $0 \leq i \leq m n-1$, of $D_{P_{c}}$, which are given by $\bar{a}_{i}=(-1)^{i} c^{\sum_{j=i+1}^{m n-1} p^{j}}$. Now Proposition 2.1.1 gives the result. Indeed,

$$
\begin{aligned}
P_{c}^{-1}\left(P_{c}(x)\right) & =\sum_{i=0}^{m n-1} \frac{(-1)^{i} c^{\sum_{j=i+1}^{m n-1} p^{j}}\left(x^{p}+c x\right)^{p^{i}}}{c^{n\left(p^{m}-1\right) /(p-1)}+(-1)^{m n-1}} \\
& =\sum_{i=0}^{m n-1} \frac{(-1)^{i} c^{\sum_{j=i+1}^{m n-1} p^{j}}\left(x^{p^{i+1}}+c^{p^{i}} x^{p^{i}}\right)}{c^{n\left(p^{m}-1\right) /(p-1)}+(-1)^{m n-1}} \\
& =\sum_{i=1}^{m n} \frac{(-1)^{i-1} c^{\sum_{j=i}^{m n-1} p^{j}} x^{p^{i}}}{c^{n\left(p^{m}-1\right) /(p-1)}+(-1)^{m n-1}}+\sum_{i=0}^{m n-1} \frac{(-1)^{i} c^{\sum_{j=i}^{m n-1} p^{j}} x^{p^{i}}}{c^{n\left(p^{m}-1\right) /(p-1)}+(-1)^{m n-1}} \\
& =x+\sum_{i=1}^{m n-1} \frac{\left((-1)^{i-1} c^{\sum_{j=i}^{m n-1} p^{j}}+(-1)^{i} c^{\sum_{j=i}^{m n-1} p^{j}}\right) x^{p^{i}}}{c^{n\left(p^{m}-1\right) /(p-1)}+(-1)^{m n-1}} \\
& =x .
\end{aligned}
$$

Similarly one can show that $P_{c}\left(P_{c}^{-1}(x)\right)=x$. At this point we have exhausted all the possibilities for $c$ and so the proof is complete.

Remark 3.2.2. Of course one can use Theorem 2.1.5 to obtain the result in Case 1, as we have done in Example 2.1.8 with $\delta=n-1$ there. As a way of comparison, and in the style of Remark 3.4 in [81], here we give an explanation of the "direct" method the authors used prior to obtaining the aforementioned results. First note that $P_{c}(\operatorname{ker}(T)) \subseteq \operatorname{ker}(T)$. As the set of p-polynomials over $\mathbb{F}_{q}$ permutating a subspace of $\mathbb{F}_{q^{n}}$ forms a group under composition, assume $P_{c}^{-1}(x)=\gamma \sum_{i=0}^{m n-1} d_{i} x^{p^{i}}$ for some $\gamma \in \mathbb{F}_{p}^{*}$ and some elements $d_{i} \in \mathbb{F}_{q}$, whenever $P_{c}$ is invertible on $\operatorname{ker}(T)$. Write $P_{c}^{-1}(x)=\gamma \sum_{j=0}^{m-1} \sum_{k=0}^{n-1} d_{k m+j} x^{p^{k m+j}}$. For any $x \in \operatorname{ker}(T)$, and using the fact that $x-$ 


$$
\begin{aligned}
& T(x)=-x^{p^{m}}-x^{p^{2 m}}-\cdots-x^{p^{(n-1) m}}, \text { assume } \\
& P_{c}^{-1}\left(P_{c}(x)\right)=\gamma \sum_{j=0}^{m-1} \sum_{k=0}^{n-1} d_{k m+j}\left(x^{p}+c x\right)^{p^{k m+j}} \\
& =\gamma\left(d_{n m-1}+d_{0} c\right) x+\gamma \sum_{k=1}^{n-1}\left(d_{k m-1}+d_{k m} c\right) x^{p^{k m}} \\
& \quad+\gamma \sum_{j=1}^{m-1} \sum_{k=0}^{n-1}\left(d_{k m+j-1}+d_{k m+j} c^{p^{j}}\right) x^{p^{k m+j}} \\
& =\gamma\left(d_{n m-1}+d_{0} c+1\right) x-\gamma T(x) \\
& =\gamma\left(d_{n m-1}+d_{0} c+1\right) x \\
& =x .
\end{aligned}
$$

We must have $d_{n m-1}+d_{0} c+1 \neq 0$ and the system of equations

$$
\left\{\begin{array}{l}
d_{i-1}+d_{i} c^{p^{i}}=0, \text { if } m \nmid i \\
d_{i-1}+d_{i} c=-1, \text { if } m \mid i,
\end{array}\right.
$$

for $0<i<m n$, with solution given by $d_{k m+j}=a_{j} c d_{k m}=a_{j} b_{k}$, where

$$
b_{k}:=\left(-a_{m-1}\right)^{k} \delta-\sum_{l=0}^{k-1}\left(-a_{m-1}\right)^{l}
$$

with $\delta:=c d_{0}$ to be determined, for $0 \leq j \leq m-1$ and $0 \leq k \leq n-1$. Note that if $\delta \in \mathbb{F}_{p}$, then each $b_{k} \in \mathbb{F}_{p}$ as $a_{m-1}=-N_{q \mid p}\left(-c^{-1}\right) \in \mathbb{F}_{p}$, where $N_{q \mid p}: \mathbb{F}_{q} \rightarrow \mathbb{F}_{p}$ is the absolute norm function given by $N_{q \mid p}(y)=y^{(q-1) /(p-1)}$. Hence if $d_{n m-1}+d_{0} c+1 \neq 0$, we set $\gamma=\left(d_{n m-1}+d_{0} c+1\right)^{-1}=\left(a_{m-1} b_{n-1}+\delta+1\right)^{-1} \in \mathbb{F}_{p}^{*}$. It is left to the reader to check that $P_{c}^{-1}\left(P_{c}(x)\right)=P_{c}\left(P_{c}^{-1}(x)\right)=x$ on $\operatorname{ker}(T)$ if $\delta \in \mathbb{F}_{p}$ and $a_{m-1} b_{n-1}+\delta+1 \neq 0$, 
in which case the inverse of $P_{c}$ on $\operatorname{ker}(T)$ is given by

$$
P_{c}^{-1}(x)=\left(a_{m-1} b_{n-1}+\delta+1\right)^{-1} \sum_{j=0}^{m-1} \sum_{k=0}^{n-1} a_{j} b_{k} x^{p^{k m+j}} .
$$

Now for Case 1, if we assume $c^{\left(p^{m}-1\right) /(p-1)}=(-1)^{m}$, then $a_{m-1}=-1$; hence $b_{k}=\delta-k$ and $a_{m-1} b_{n-1}+\delta+1=-(\delta-n+1)+\delta+1=n \neq 0$ since $p \nmid n$ by assumption.

Corollary 3.2.3 (Lemma 3.3, [81]). Let $q=2^{m}$ and $n$ be odd. Let $P_{c}(x)=$ $x^{2}+c x$ for any $c \in \mathbb{F}_{q}^{*}$. Then $P_{c}$ can induce a permutation of $\operatorname{ker}(T)$ and one of the polynomials that can induce its inverse map is

$$
P_{c}^{-1}(x)=\sum_{j=0}^{m-1} c^{-\left(2^{j+1}-1\right)}\left(\sum_{k=0}^{\frac{n-1}{2}} x^{q^{2 k}}\right)^{2^{j}} .
$$

Proof. As $2 \nmid n$ and $c^{2^{m}-1}=1$, this is Case 1 of Lemma 3.2.1 with $\delta=1$.

Denote by $N_{q \mid p}: \mathbb{F}_{q} \rightarrow \mathbb{F}_{p}$ the absolute norm function given by $N_{q \mid p}(y)=$ $y^{(q-1) /(p-1)}$.

Theorem 3.2.4. Let $q=p^{m}$ be a power of a prime $p$, let $n$ be a positive integer, let $a \in \mathbb{F}_{q}^{*}$, and assume that $g \in \mathbb{F}_{q}[x]$ is such that $\bar{f}(x):=a x^{p}+x g(x)$ induces a permutation of $\mathbb{F}_{q}$ and $\varphi_{y}(x):=a x^{p}+x g(y)$ induces a permutation of $\operatorname{ker}(T)=$ $\left\{\beta^{q}-\beta \mid \beta \in \mathbb{F}_{q^{n}}\right\}$, for each $y \in \mathbb{F}_{q}$. Then

$$
f(x):=a x^{p}+x g(T(x))
$$

induces a permutation of $\mathbb{F}_{q^{n}}$. Let $\bar{f}^{-1}$ be the inverse of the permutation $\left.\bar{f}\right|_{\mathbb{F}_{q}}$.

(a) If $x \in \mathbb{F}_{q^{n}}$ is such that $g\left(\bar{f}^{-1}(T(x))\right)=0$, the preimage of $x$ under $f$ is given 
by

$$
f^{-1}(x)=\left(\frac{x}{a}\right)^{q^{n} / p}
$$

Otherwise, if $p \mid n$ or $x \in \mathbb{F}_{q^{n}}$ is such that $\varphi_{\bar{f}^{-1}(T(x))}$ permutes $\mathbb{F}_{q}$ (equivalently, $\left.N_{q \mid p}\left(g\left(\bar{f}^{-1}(T(x))\right) / a\right) \neq(-1)^{m}\right)$, then $\varphi_{\bar{f}^{-1}(T(x))}$ permutes $\mathbb{F}_{q^{n}}$ and the preimage of $x$ under $f$ is given by

$$
f^{-1}(x)=\sum_{i=0}^{m n-1} \frac{(-1)^{i} a^{\left(p^{i}-1\right) /(p-1)} g\left(\bar{f}^{-1}(T(x))\right)^{\sum_{j=i+1}^{m n-1} p^{j}}}{N_{q \mid p}\left(g\left(\bar{f}^{-1}(T(x))\right)^{n}\right)-N_{q \mid p}\left((-a)^{n}\right)} x^{p^{i}} .
$$

(b) Otherwise (if $p \nmid n$ and $x \in \mathbb{F}_{q^{n}}$ is such that $\left.N_{q \mid p}\left(g\left(\bar{f}^{-1}(T(x))\right) / a\right)=(-1)^{m}\right)$, the preimage of $x$ under $f$ is given by

$$
\left.f^{-1}(x)=n^{-1}\left(\bar{f}^{-1}(T(x))-\sum_{j=0}^{m-1} \frac{(-1)^{j} a^{\left(p^{j}-1\right) /(p-1)}}{g\left(\bar{f}^{-1}(T(x))\right)^{\left(p^{j+1}-1\right) /(p-1)}}\left(\sum_{k=1}^{n-1} k\left(x^{q^{k}}-n^{-1} T(x)\right)\right)\right)^{p^{j}}\right) .
$$

Proof. The fact that $f$ permutes $\mathbb{F}_{q^{n}}$ under the assumptions follows from Theorem 3.1 .13 with $H(x)=a x^{p}$.

(a) In this case we have $f^{-1}(x)=\varphi_{\bar{f}^{-1}(T(x))}^{-1}(x)$ by Corollary 3.1.14. If $y \in \mathbb{F}_{q}$ is such that $g(y)=0$, then $\varphi_{y}(x)=a x^{p}$ is a permutation of $\mathbb{F}_{q^{n}}$, and $\varphi_{y}^{-1}(x)=(x / a)^{q^{n} / p}$. Otherwise, noting that $\varphi_{y}(x)=a P_{g(y) / a}(x)$, where $P_{c}(x)=x^{p}+c x$ from Lemma 3.2.1, and substituting $y$ with $\bar{f}^{-1}(T(x))$, we get $f^{-1}(x)=P_{g\left(\bar{f}^{-1}(T(x))\right) / a}^{-1}(x / a)$, where $P_{c}^{-1}$, with $c=g\left(\bar{f}^{-1}(T(x))\right) / a$, is given in Case 2 of Lemma 3.2.1. Thus we obtain

$$
f^{-1}(x)=\frac{\sum_{i=0}^{m n-1}(-1)^{i}\left(\frac{g\left(\bar{f}^{-1}(T(x))\right)}{a}\right)^{\sum_{j=i+1}^{m n-1} p^{j}}\left(\frac{x}{a}\right)^{p^{i}}}{N_{q \mid p}\left(\frac{g\left(\bar{f}^{-1}(T(x))\right)}{a}\right)^{n}+(-1)^{m n-1}} .
$$

Now since $N_{q \mid p}(a)^{n}(-1)^{m n-1}=-N_{q \mid p}(-a)^{n}$ and $N_{q \mid p}(a)^{n} a^{-\sum_{j=i}^{m n-1} p^{j}}=a^{\sum_{j=0}^{m n-1} p^{j}-\sum_{j=i}^{m n-1} p^{j}}=$ 
$a^{\sum_{j=0}^{i-1} p^{j}}=a^{\left(p^{i}-1\right) /(p-1)}$, the result follows.

(b) Assume that $y \in \mathbb{F}_{q}$ is such that $N_{q \mid p}(g(y) / a)=(-1)^{m}$. Then $\varphi_{y}(x)=$ $a x^{p}+x g(y)=a\left(x^{p}+x g(y) / a\right)=a P_{g(y) / a}(x)$ where $P_{c_{y}}, c_{y}=g(y) / a \in \mathbb{F}_{q}^{*}$, is a permutation of $\operatorname{ker}(T)$ in Case 1 of Lemma 3.2.1. Hence $\left.\varphi_{y}^{-1}\right|_{\operatorname{ker}(T)}(x)=P_{g(y) / a}^{-1}(x / a)$, where $P_{g(y) / a}^{-1}$ is given in Case 1 of Lemma 3.2.1. Then substituting $y$ with $\bar{f}^{-1}(T(x))$ in $\left.\varphi_{y}^{-1}\right|_{\operatorname{ker}(T)}$ and then using Corollary 3.1.14 together with Case 1 of Lemma 3.2.1 with $c=g\left(\bar{f}^{-1}(T(x))\right) / a$ and $\delta=0$, we get

$$
\begin{aligned}
f^{-1}(x)= & n^{-1} \bar{f}^{-1}(T(x))+P_{g\left(\bar{f}^{-1}(T(x))\right) / a}^{-1}\left(\frac{x-n^{-1} T(x)}{a}\right) \\
= & n^{-1} \bar{f}^{-1}(T(x)) \\
& -n^{-1} \sum_{j=0}^{m-1} \sum_{k=1}^{n-1} k(-1)^{j}\left(\frac{a}{g\left(\bar{f}^{-1}(T(x))\right)}\right)^{\frac{p^{j+1}-1}{p-1}}\left(\frac{x-n^{-1} T(x)}{a}\right)^{q^{k} p^{j}} \\
= & n^{-1} \bar{f}^{-1}(T(x)) \\
& -n^{-1} \sum_{j=0}^{m-1} \sum_{k=1}^{n-1} k(-1)^{j}\left(\frac{a}{g\left(\bar{f}^{-1}(T(x))\right)}\right)^{\frac{p^{j+1}-1}{p-1}} a^{-p^{j}}\left(x^{q^{k}}-n^{-1} T(x)\right)^{p^{j}} \\
= & \left.n^{-1}\left(\bar{f}^{-1}(T(x))-\sum_{j=0}^{m-1} \frac{(-1)^{j} a^{\left(p^{j}-1\right) /(p-1)}}{g\left(\bar{f}^{-1}(T(x))\right)^{\left(p^{j+1}-1\right) /(p-1)}}\left(\sum_{k=1}^{n-1} k\left(x^{q^{k}}-n^{-1} T(x)\right)\right)\right)^{p^{j}}\right),
\end{aligned}
$$

as required.

Remark 3.2.5. One may find several $g \in \mathbb{F}_{q}[x]$ such that $\varphi_{y}(x)=a x^{p}+x g(y)=$ $a\left(x^{p}+x g(y) / a\right)$ induces a permutation of $\operatorname{ker}(T)$ for each $y \in \mathbb{F}_{q}$. For instance if we let $m$ be even and $n$ be such that $p \nmid n$ and $\operatorname{gcd}(n, p-1)=1$, then $\varphi_{y}(x)=$ $a\left(x^{p}+x g(y) / a\right)$ induces a permutation of $\operatorname{ker}(T)$ for each $g \in \mathbb{F}_{q}[x]$ and each $y \in \mathbb{F}_{q}$. Indeed, from Lemma 3.2.1 it follows that $\varphi_{y}$ induces a permutation of $\operatorname{ker}(T)$ if and only if $N_{q \mid p}(g(y) / a)=1$ or $N_{q \mid p}(g(y) / a)^{n} \neq 1$. Noting that $\operatorname{gcd}(n, p-1)=1$ implies that there does not exist an $n$-th root of unity in $\mathbb{F}_{p} \backslash\{1\}$, then if $N_{q \mid p}(g(y) / a) \neq 1$, 
we get $N_{q \mid p}(g(y) / a)^{n} \neq 1$ as required. For example we can pick $g(x)=x^{p-1}$, and if $a \neq-1$, then $\bar{f}(x):=a x^{p}+x g(x)=(a+1) x^{p}$ permutes $\mathbb{F}_{q}$, having inverse (on $\mathbb{F}_{q}$ ) given by $\bar{f}^{-1}(x)=(x /(a+1))^{q / p}$. As another example we can let $g(x)=x^{q-2} L(x)$ where $L \in \mathbb{F}_{q}[x]$ is any p-polynomial such that $\left.\bar{f}\right|_{\mathbb{F}_{q}}(x)=a x^{p}+x g(x)=a x^{p}+L(x)$ is a permutation of $\mathbb{F}_{q}$. Then one can use for instance Proposition 2.1.1 to obtain the inverse of $\left.\bar{f}\right|_{\mathbb{F}_{q}}$.

Corollary 3.2.6. Using the same notations and assumptions of Theorem 3.2.4, the compositional inverse of $f$ on $\mathbb{F}_{q^{n}}[x]$ is given by

$$
\begin{aligned}
f^{-1}(x) & =\left(1-g\left(\bar{f}^{-1}(T(x))\right)^{q-1}\right)\left(\frac{x}{a}\right)^{q^{n} / p} \\
& +g\left(\bar{f}^{-1}(T(x))\right)^{q-1}\left(\left(\frac{g\left(\bar{f}^{-1}(T(x))\right)}{a}\right)^{(q-1) /(p-1)}-(-1)^{m}\right)^{p-1} \\
& \cdot \sum_{i=0}^{m n-1} \frac{(-1)^{i} a^{\left(p^{i}-1\right) /(p-1)} g\left(\bar{f}^{-1}(T(x))\right)^{\sum_{j=i+1}^{m n-1} p^{j}}}{g\left(\bar{f}^{-1}(T(x))\right)^{n(q-1) /(p-1)}-(-a)^{n(q-1) /(p-1)}} x^{p^{i}} \\
& +\left(1-\left(\left(\frac{g\left(\bar{f}^{-1}(T(x))\right)}{a}\right)^{(q-1) /(p-1)}-(-1)^{m}\right)^{p-1}\right) \\
& \cdot n^{p-2}\left(\bar{f}^{-1}(T(x))-\sum_{j=0}^{m-1} \frac{(-1)^{j} a^{\left(p^{j}-1\right) /(p-1)}}{g\left(\bar{f}^{-1}(T(x))\right)^{\left(p^{j+1}-1\right) /(p-1)}}\left(\sum_{k=1}^{n-1} k\left(x^{q^{k}}-n^{p-2} T(x)\right)\right)^{p^{j}}\right) .
\end{aligned}
$$

Proof. We put the results of Theorem 3.2.4 (a) and (b) together. This is a step function where only one of the three terms is non-zero at a time. Denote $c_{x}:=$ $g\left(\bar{f}^{-1}(T(x))\right) / a$. The first term of the expression is non-zero only if $c_{x}=0$ corresponding to the result in (a), while the second is non-zero only if $c_{x}^{(q-1) /(p-1)} \neq 0,(-1)^{m}$, given in $(a)$ as well. The third term is non-zero only if $c_{x}^{(q-1) /(p-1)}=(-1)^{m}$ and $p \nmid n$, which is given in (b). Because $\varphi_{c_{x}}$ is a permutation of $\operatorname{ker}(T)$, then by Lemma 3.2.1 these are all the possibilities of $c_{x}$; hence we are done. 
In Theorem 3.2.4, if we let $p=2, n$ be odd, $g(x)=x$, and $a \in \mathbb{F}_{q} \backslash\{0,1\}$, then we get $f(x)=a x^{2}+x T(x)=x(T(x)+a x)$, the compositional inverse of which was given in [23]. Similarly, if instead we let $g(x)=L(x)+a x$ for some additive $L$ over $\mathbb{F}_{q}$ and some $a \in \mathbb{F}_{q}^{*}$, then we get $f(x)=a x^{2}+x g(T(x))=x(L(T(x))+a T(x)+a x)$, whose compositional inverse was obtained in [81]. Thus these compositional inverses should follow from Corollary 3.2.6.

\subsection{Explicit compositional inverse of a second class}

We further demonstrate the utility of Theorem 1.0.2 by obtaining the explicit compositional inverse of a class of permutation polynomials generalizing that of a linearized class given in Theorem 2.2 of [79], where the corresponding inverse was obtained. Our result is presented in Theorem 1.0.10. See also Lemma 3.3.3 which implies the aforementioned result by giving the compositional inverse of a more general class of linearized permutation polynomials. In particular, the method of our proof, which is an application of Theorem 1.0.2, seems considerably less complicated than that employed in the aforementioned paper; the latter consisted of a direct application of Proposition 2.1.1 and the computation of determinants. First we need the following two lemmas.

Lemma 3.3.1. Let $\alpha \in \mathbb{F}_{q^{n}}$ and denote $T_{\alpha}(x):=T(\alpha x)$. Then $\varphi(x):=x^{q}-x$ induces a bijection from $\operatorname{ker}\left(T_{\alpha}\right)$ to $\operatorname{ker}(T)$, in $\mathbb{F}_{q^{n}}$, if and only if $T(\alpha) \neq 0$. In the case that $T(\alpha) \neq 0$, one of the polynomials, over $\mathbb{F}_{q^{n}}$, inducing the inverse map of $\left.\varphi\right|_{\operatorname{ker}\left(T_{\alpha}\right)}$, is given by

$$
\left.\varphi^{-1}\right|_{\operatorname{ker}(T)}(x)=T(\alpha)^{-1} \sum_{k=0}^{n-1} \sum_{j=0}^{k} \alpha^{q^{j}} x^{q^{k}} .
$$

Proof. If we assume that $T(\alpha)=0$, then $T_{\alpha}(1)=T(\alpha)=0$ and $\varphi(1)=0$; hence 
$1 \in \operatorname{ker}(\varphi) \cap \operatorname{ker}\left(T_{\alpha}\right)$ and $\left.\varphi\right|_{\operatorname{ker}\left(T_{\alpha}\right)}$ is not bijective. Now assume $T(\alpha) \neq 0$. Since $\operatorname{ker}(T)=\left\{x^{q}-x \mid x \in \mathbb{F}_{q^{n}}\right\}=\operatorname{im}(\varphi)$, then $\varphi\left(\operatorname{ker}\left(T_{\alpha}\right)\right) \subseteq \operatorname{ker}(T)$. Note that $\operatorname{im}\left(T_{\alpha}\right) \subseteq$ $\mathbb{F}_{q}$ (because $\operatorname{im}(T)=\mathbb{F}_{q}$ ). Moreover, for any $c \in \mathbb{F}_{q}$, we have $c=c T(\alpha)^{-1} T(\alpha)=$ $T\left(c T(\alpha)^{-1} \alpha\right) \in \operatorname{im}\left(T_{\alpha}\right)$; hence $\operatorname{im}\left(T_{\alpha}\right)=\operatorname{im}(T)=\mathbb{F}_{q}$. It follows that $\left|\operatorname{ker}\left(T_{\alpha}\right)\right|=$ $|\operatorname{ker}(T)|$. To show that $\left.\varphi\right|_{\operatorname{ker}\left(T_{\alpha}\right)}$ is injective, let $k \in \operatorname{ker}(\varphi) \cap \operatorname{ker}\left(T_{\alpha}\right)$. In particular, $\varphi(k)=k^{q}-k=0$ implies $k \in \mathbb{F}_{q}$. Then $T_{\alpha}(k)=T(\alpha k)=k T(\alpha)=0$ gives $k=0$ as required. Thus $\varphi$ is bijective from $\operatorname{ker}\left(T_{\alpha}\right)$ to $\operatorname{ker}(T)$. Next we prove that the given $\left.\varphi^{-1}\right|_{\operatorname{ker}(T)}$ induces the inverse map of $\left.\varphi\right|_{\operatorname{ker}\left(T_{\alpha}\right)}$. Assuming $x \in \operatorname{ker}\left(T_{\alpha}\right)$, we have

$$
\begin{aligned}
\left.\varphi^{-1}\right|_{\operatorname{ker}(T)}(\varphi(x)) & =T(\alpha)^{-1} \sum_{k=0}^{n-1} \sum_{j=0}^{k} \alpha^{q^{j}}\left(x^{k^{k+1}}-x^{q^{k}}\right) \\
& =T(\alpha)^{-1}\left(\sum_{k=1}^{n} \sum_{j=0}^{k-1} \alpha^{q^{j}} x^{q^{k}}-\sum_{k=0}^{n-1} \sum_{j=0}^{k} \alpha^{q^{j}} x^{q^{k}}\right) \\
& =\left(1-T(\alpha)^{-1} \alpha\right) x+T(\alpha)^{-1} \sum_{k=1}^{n-1}\left(\sum_{j=0}^{k-1} \alpha^{q^{j}}-\sum_{j=0}^{k} \alpha^{q^{j}}\right) x^{q^{k}} \\
& =\left(1-T(\alpha)^{-1} \alpha\right) x-T(\alpha)^{-1} \sum_{k=1}^{n-1}(\alpha x)^{q^{k}} \\
& =\left(1-T(\alpha)^{-1} \alpha\right) x-T(\alpha)^{-1}\left(T_{\alpha}(x)-\alpha x\right) \\
& =x-T(\alpha)^{-1} T_{\alpha}(x) \\
& =x,
\end{aligned}
$$

as required.

Remark 3.3.2. We used Theorem 2.1.5 to obtain the coefficients $\bar{c}=\left(c_{0} c_{1} \cdots c_{n-1}\right)$ of $\left.\varphi^{-1}\right|_{\operatorname{ker}\left(T_{\alpha}\right)}$. That is, we obtained a solution, $\bar{c}$, to the linear equation

$$
\bar{c} D_{\varphi}=v_{q, n}\left(\mathrm{id}-T(\alpha)^{-1} T_{\alpha}\right)=-T(\alpha)^{-1}\left(\alpha-T(\alpha), \alpha^{q}, \alpha^{q^{2}}, \ldots, \alpha^{q^{n-1}}\right),
$$


where $T(\alpha)^{-1} T_{\alpha}$ is idempotent with kernel $\operatorname{ker}\left(T_{\alpha}\right)$, and $D_{\varphi}$ is the $n \times n$ associate Dickson matrix of $\varphi(x)=x^{q}-x$ given by

$$
D_{\varphi}=\left(\begin{array}{cccccc}
-1 & 1 & 0 & 0 & \cdots & 0 \\
0 & -1 & 1 & 0 & \cdots & 0 \\
\vdots & \vdots & \vdots & \vdots & & \vdots \\
1 & 0 & 0 & 0 & \cdots & -1
\end{array}\right)
$$

The following lemma gives the compositional inverse of a class of linearized permutation polynomials generalizing that whose inverse was recently obtained in Theorem 2.2, [79]. See Corollary 3.3.5. The method utilized here to obtain such a result, as an application of Theorem 1.0.2, seems much less complicated than that employed in [79].

Lemma 3.3.3. Let $\alpha \in \mathbb{F}_{q^{n}}$, and define the polynomial

$$
f(x):=x^{q}-x+T(\alpha x) \in \mathbb{F}_{q^{n}}[x] .
$$

Then the following two results hold.

(a) $f$ is a permutation polynomial over $\mathbb{F}_{q^{n}}$ if and only if $T(\alpha) \neq 0$, and the characteristic, $p$, of $\mathbb{F}_{q}$, does not divide $n$.

(b) If $T(\alpha) \neq 0$ and $p \nmid n$ (equivalently, $f$ is a permutation polynomial over $\mathbb{F}_{q^{n}}$ ), then the compositional inverse of $f$ over $\mathbb{F}_{q^{n}}$ is

$$
f^{-1}(x)=T(\alpha)^{-1} n^{-1}(T(x)+B(x)),
$$


where the coefficients of $B(x)=\sum_{k=0}^{n-1} b_{k} x^{q^{k}} \in \mathbb{F}_{q^{n}}[x]$ are given by

$$
b_{k}=\sum_{j=1}^{n-1} j \alpha^{q^{j}}-n \sum_{l=k+1}^{n-1} \alpha^{q^{l}}, \quad 0 \leq k \leq n-1 .
$$

Proof. (a) As done in Lemma 3.3.1, denote $\varphi(x):=x^{q}-x, T_{\alpha}(x):=T(\alpha x)$. The reader can check that $\varphi \circ T_{\alpha}=T \circ \varphi=0$. From the proof of Lemma 3.3.1 we know that $\operatorname{im}\left(T_{\alpha}\right)=\operatorname{im}(T)=\mathbb{F}_{q}$. Thus letting $\psi=T_{\alpha}, \bar{\psi}=T, g(x)=x, h=1$, and $\varphi$ as before, we see that the polynomial, $f$, is an instance of Theorem 1.0.1. Hence, the permutability of $f$ boils down to whether or not both conditions (i), (ii), of Theorem 1.0.1, are satisfied. From the proof of Lemma 3.3.1, we know that $\operatorname{ker}(\varphi) \cap \operatorname{ker}\left(T_{\alpha}\right)=\{0\}$ if and only if $T(\alpha) \neq 0$. Moreover, for all $y \in \mathbb{F}_{q}$, we have $\bar{f}(y)=\varphi(y)+T(y)=n y$; thus $\bar{f}$ permutes $\operatorname{im}\left(T_{\alpha}\right)=\operatorname{im}(T)=\mathbb{F}_{q}$ if and only if $p \nmid n$. Hence, by Theorem 1.0.1, $f$ is a permutation polynomial if and only if $T(\alpha) \neq 0$ and $p \nmid n$.

(b) We wish to apply Theorem 1.0.2. However in our specific case one can show that both $S_{T_{\alpha}}, S_{T}=\mathbb{F}_{q^{n}}$ which $\varphi$ does not permute. As we shall see, we can bypass this problem by considering instead the permutation polynomial $H:=T(\alpha)^{-1} f$. Noting that $\varphi \circ T(\alpha)^{-1} T_{\alpha}=n^{-1} T \circ \varphi=0$ and $\operatorname{im}\left(T(\alpha)^{-1} T_{\alpha}\right)=\operatorname{im}\left(n^{-1} T\right)=\mathbb{F}_{q}$, we see that $H$ is an instance of Theorem 1.0.1 with $\psi=T(\alpha)^{-1} T_{\alpha}, \bar{\psi}=n^{-1} T, h=T(\alpha)^{-1}$, and $\varphi, g$, as before. We have, for all $y \in \mathbb{F}_{q}, \bar{H}(y)=\varphi(y)+n^{-1} T(y)=y$, thus permuting $\mathbb{F}_{q}=\operatorname{im}(\psi)=\operatorname{im}(\bar{\psi})$. The reader can check that both $\psi=T(\alpha)^{-1} T_{\alpha}, \bar{\psi}=n^{-1} T$, are idempotent. Then $S_{\psi}=\operatorname{ker}(\psi)=\operatorname{ker}\left(T_{\alpha}\right)$ and $S_{\bar{\psi}}=\operatorname{ker}(\bar{\psi})=\operatorname{ker}(T)$; hence $\left|S_{\psi}\right|=$ $\left|S_{\bar{\psi}}\right|$ (since $\left|\operatorname{ker}\left(T_{\alpha}\right)\right|=|\operatorname{ker}(T)|$ because the images of $T_{\alpha}, T$, are equal). Moreover, from the proof of (a) we know that $\operatorname{ker}(\varphi) \cap S_{\psi}=\operatorname{ker}(\varphi) \cap \operatorname{ker}\left(T_{\alpha}\right)=\{0\}$. In particular $\operatorname{ker}(\varphi) \cap \psi\left(S_{\psi}\right)=\{0\}$ since $\psi\left(S_{\psi}\right) \subseteq S_{\psi}$. Thus $H$ satisfies the assumptions of Theorem 
1.0.2, which, after the appropriate substitutions and subsequent simplification, yields

$$
H^{-1}(x)=n^{-1} T(x)+\left.\varphi^{-1}\right|_{\operatorname{ker}(T)}\left(T(\alpha)\left(x-n^{-1} T(x)\right)\right) .
$$

Now using the fact that $f^{-1}(x)=H^{-1}\left(T(\alpha)^{-1} x\right)$, we get

$$
f^{-1}(x)=T(\alpha)^{-1} n^{-1} T(x)+\left.\varphi^{-1}\right|_{\operatorname{ker}(T)}\left(x-n^{-1} T(x)\right) .
$$

From Lemma 3.3.1, we may take $\left.\varphi^{-1}\right|_{\operatorname{ker}(T)}(x)=T(\alpha)^{-1} \sum_{k=0}^{n-1} \sum_{j=0}^{k} \alpha^{q^{j}} x^{q^{k}}$. Thus,

$$
\begin{aligned}
\left.\varphi^{-1}\right|_{\operatorname{ker}(T)}\left(x-n^{-1} T(x)\right) & =T(\alpha)^{-1} \sum_{k=0}^{n-1} \sum_{j=0}^{k} \alpha^{q^{j}}\left(x-n^{-1} T(x)\right)^{q^{k}} \\
& =T(\alpha)^{-1}\left(\sum_{k=0}^{n-1} \sum_{j=0}^{k} \alpha^{q^{j}} x^{q^{k}}-n^{-1} \sum_{l=0}^{n-1} \sum_{k=0}^{n-1} \sum_{j=0}^{k} \alpha^{q^{j}} x^{q^{l}}\right) \\
& =T(\alpha)^{-1}\left(\sum_{k=0}^{n-1} \sum_{j=0}^{k} \alpha^{q^{j}} x^{q^{k}}-n^{-1} \sum_{l=0}^{n-1} \sum_{j=0}^{n-1}(n-j) \alpha^{q^{j}} x^{q^{l}}\right) \\
& =T(\alpha)^{-1}\left(\sum_{k=0}^{n-1} \sum_{j=0}^{k} \alpha^{q^{j}} x^{q^{k}}-n^{-1} \sum_{l=0}^{n-1}\left(n T(\alpha)-\sum_{j=0}^{n-1} j \alpha^{q^{j}}\right) x^{q^{l}}\right) \\
& =T(\alpha)^{-1} \sum_{k=0}^{n-1}\left(\sum_{j=0}^{k} \alpha^{q^{j}}-T(\alpha)+n^{-1} \sum_{j=0}^{n-1} j \alpha^{q^{j}}\right) x^{q^{k}} \\
& =T(\alpha)^{-1} \sum_{k=0}^{n-1}\left(-\sum_{j=k+1}^{n-1} \alpha^{q^{j}}+n^{-1} \sum_{j=0}^{n-1} j \alpha^{q^{j}}\right) x^{q^{k}} \\
& =T(\alpha)^{-1} n^{-1} B(x) .
\end{aligned}
$$

The result now follows immediately.

Remark 3.3.4. To further convince the reader of the correctness of the result in (b), we give a second, more direct, proof. That is, we show $f^{-1}(f(x))=x$. Since $B$ is a 
q-polynomial, it follows that

$$
f^{-1}(f(x))=T(\alpha)^{-1}\left(T(\alpha x)+n^{-1}\left(B\left(x^{q}\right)-B(x)+B(1) T(\alpha x)\right)\right) .
$$

We have

$$
\begin{aligned}
n^{-1} B(1) & =\sum_{k=0}^{n-1}\left(n^{-1} \sum_{j=1}^{n-1} j \alpha^{q^{j}}-\sum_{j=k+1}^{n-1} \alpha^{q^{j}}\right)=\sum_{j=1}^{n-1} j \alpha^{q^{j}}-\sum_{k=0}^{n-1} \sum_{j=k+1}^{n-1} \alpha^{q^{j}} \\
& =\sum_{j=1}^{n-1} j \alpha^{q^{j}}-\sum_{k=0}^{n-1}\left(T(\alpha)-\sum_{j=0}^{k} \alpha^{q^{j}}\right)=\sum_{j=1}^{n-1} j \alpha^{q^{j}}-n T(\alpha)+\sum_{k=0}^{n-1} \sum_{j=0}^{k} \alpha^{q^{j}} \\
& =\sum_{j=1}^{n-1} j \alpha^{q^{j}}-n T(\alpha)+\sum_{j=0}^{n-1}(n-j) \alpha^{q^{j}} \\
& =0 .
\end{aligned}
$$

\section{Additionally}

$$
\begin{aligned}
n^{-1}\left(B\left(x^{q}\right)-B(x)\right) & =n^{-1}\left(\sum_{k=1}^{n} b_{k-1} x^{q^{k}}-\sum_{k=0}^{n-1} b_{k} x^{q^{k}}\right) \\
& =n^{-1}\left(\left(b_{n-1}-b_{0}\right) x+\sum_{k=1}^{n-1}\left(b_{k-1}-b_{k}\right) x^{q^{k}}\right) \\
& =\sum_{j=1}^{n-1} \alpha^{q^{j}} x+\sum_{k=1}^{n-1}\left(-\sum_{j=k}^{n-1} \alpha^{q^{j}}+\sum_{j=k+1}^{n-1} \alpha^{q^{j}}\right) x^{q^{k}} \\
& =\sum_{j=1}^{n-1} \alpha^{q^{j}} x-\sum_{k=1}^{n-1}(\alpha x)^{q^{k}} \\
& =(T(\alpha)-\alpha) x-(T(\alpha x)-\alpha x) \\
& =T(\alpha) x-T(\alpha x) .
\end{aligned}
$$

Now the result, $f^{-1}(f(x))=x$, follows soon.

Note in Theorem 1.0.10 that if we let $G(x)=x$ and $c=1$, we obtain Lemma 
3.3.3. We are now ready to prove Theorem 1.0.10.

Proof of Theorem 1.0.10. If $T(\alpha)=0$, then $F \circ T=F(0)$ implying $F$ is not injective. If $p \mid n$, then $T \circ F=0$ implying $F$ is not surjective. As a result, if $F$ permutes $\mathbb{F}_{q^{n}}$, necessarily $T(\alpha) \neq 0$ and $p \nmid n$. Assume $T(\alpha) \neq 0$ and $p \nmid n$. We have that $f(x)=x^{q}-x+T(\alpha x)$ from Lemma 3.3.3 (b) permutes $\mathbb{F}_{q^{n}}$, and $f \circ T_{\alpha}=T(\alpha) n^{-1} T \circ f=T(\alpha) T_{\alpha}$. Moreover, $\operatorname{im}\left(T_{\alpha}\right)=\operatorname{im}\left(T(\alpha) n^{-1} T\right)=\mathbb{F}_{q}$, and $T(\alpha) n^{-1} T \circ Q \circ G=0$, where $Q(x):=x^{q}-x($ since $T \circ Q=0)$. Then letting $\varphi=f$, $\psi=T_{\alpha}, \bar{\psi}=T(\alpha) n^{-1} T, g=Q \circ G$, and $h=c$, Corollary 1.0.4 implies that $F$ permutes $\mathbb{F}_{q^{n}}$. It follows that $F$ permutes $\mathbb{F}_{q^{n}}$ if and only if $T(\alpha) \neq 0$ and $p \nmid n$. In this case, Corollary 1.0.4 gives

$$
\begin{aligned}
F^{-1}(x) & =c^{-1} f^{-1}\left(x-Q \circ G \circ c^{-1} f^{-1} \circ T(\alpha) n^{-1} T(x)\right) \\
& =c^{-1} f^{-1}(x)-c^{-1} f^{-1} \circ Q \circ G \circ c^{-1} f^{-1} \circ T(\alpha) n^{-1} T(x) .
\end{aligned}
$$

From the proof of Lemma 3.3.3 (b), we know that

$$
f^{-1}(x)=T(\alpha)^{-1} n^{-1} T(x)+\left.Q^{-1}\right|_{\operatorname{ker}(T)}\left(x-n^{-1} T(x)\right),
$$

where $\left.Q^{-1}\right|_{\operatorname{ker}(T)}$ is a $q$-polynomial inducing the inverse map of $\left.Q\right|_{\operatorname{ker}\left(T_{\alpha}\right)}$. It follows that

$$
\begin{aligned}
f^{-1} \circ T(\alpha) n^{-1} T(x) & =n^{-1} T(x)+\left.Q^{-1}\right|_{\operatorname{ker}(T)}(0) \\
& =n^{-1} T(x)
\end{aligned}
$$

Hence,

$$
F^{-1}=c^{-1} f^{-1}-c^{-1} f^{-1} \circ Q \circ G \circ c^{-1} n^{-1} T .
$$


Using the fact that $T \circ Q=0$, we obtain

$$
f^{-1} \circ Q \circ G \circ c^{-1} n^{-1} T=\left.Q^{-1}\right|_{\operatorname{ker}(T)} \circ Q \circ G \circ c^{-1} n^{-1} T .
$$

Since for any $x \in \mathbb{F}_{q^{n}}$ we have $x-T(\alpha)^{-1} T(\alpha x) \in \operatorname{ker}\left(T_{\alpha}\right)$ and $Q\left(x-T(\alpha)^{-1} T(\alpha x)\right)=$ $Q(x) \in \operatorname{ker}(T)$, we get $\left.Q^{-1}\right|_{\operatorname{ker}(T)}(Q(x))=x-T(\alpha)^{-1} T(\alpha x)$, for any $x \in \mathbb{F}_{q^{n}}$. Then substituting $x$ with $G\left(c^{-1} n^{-1} T(x)\right)$ we finally obtain

$$
\begin{aligned}
F^{-1}(x) & =c^{-1} f^{-1}(x)-\left.c^{-1} Q^{-1}\right|_{\operatorname{ker}(T)} \circ Q \circ G \circ c^{-1} n^{-1} T(x) \\
& =c^{-1} T(\alpha)^{-1} n^{-1}(T(x)+B(x))-c^{-1}\left(G\left(c^{-1} n^{-1} T(x)\right)-T(\alpha)^{-1} T\left(\alpha G\left(c^{-1} n^{-1} T(x)\right)\right)\right) \\
& =c^{-1}\left[T(\alpha)^{-1} n^{-1}(T(x)+B(x))-G\left(c^{-1} n^{-1} T(x)\right)+T(\alpha)^{-1} T\left(\alpha G\left(c^{-1} n^{-1} T(x)\right)\right)\right]
\end{aligned}
$$

as required.

Corollary 3.3.5 (Theorem 2.2, [79]). Let $n$ be an odd positive integer, and let $a \in \mathbb{F}_{2^{n}}^{*}$ such that $T_{2^{n} \mid 2}\left(a^{-1}\right)=1$. Then

$$
f(x)=x^{2}+x+T_{2^{n} \mid 2}\left(\frac{x}{a}\right)
$$

is a permutation polynomial over $\mathbb{F}_{2^{n}}$ with compositional inverse

$$
f^{-1}(x)=T_{2^{n} \mid 2}(x)+B(x),
$$


where the coefficients of $B(x)=\sum_{k=0}^{n-1} b_{k} x^{2^{k}} \in \mathbb{F}_{2^{n}}[x]$ are given by

$b_{0}=a^{-2^{2}}+a^{-2^{4}}+\cdots+a^{-2^{n-1}}$

$b_{k}= \begin{cases}\left(a^{-2}+a^{-2^{3}}+\cdots+a^{-2^{k}}\right)+\left(a^{-2^{k+1}}+a^{-2^{k+3}}+\cdots+a^{-2^{n-1}}\right) & \text { if } k \text { is odd }, \\ \left(a^{-2}+a^{-2^{3}}+\cdots+a^{-2^{k-1}}\right)+\left(a^{-2^{k+2}}+a^{-2^{k+4}}+\cdots+a^{-2^{n-1}}\right) & \text { if } k \text { is even, }\end{cases}$

$1 \leq k \leq n-1$

Proof. First note that we can let $G(x)=x$ and $c=1$ in Theorem 1.0.10 in order to obtain Lemma 3.3.3. This is Lemma 3.3.3 with $q=2, n$ odd, and $\alpha=a^{-1} \in \mathbb{F}_{q^{n}}$ such that $T(\alpha)=1$. Since $2 \nmid n$ and $T(\alpha) \neq 0, f$ is a permutation polynomial by Lemma 3.3.3 (a), whereas the expression for the compositional inverse of $f$ follows from (b). Indeed, in our case of $q=2$ and $n$ odd, the coefficients of $B$ are given by

$$
\begin{aligned}
b_{k} & :=\sum_{j=1}^{n-1} j \alpha^{q^{j}}-n \sum_{\substack{l=k+1 \\
j \text { odd }}}^{n-1} \alpha^{q^{l}}=\sum_{j=1}^{k} j \alpha^{2^{j}}+\sum_{\substack{l=k+1 \\
l=k+1 \\
l \text { even }}}^{n-1}(l+1) \alpha^{2^{l}} \\
& =\sum_{\substack{j=1 \\
j \text { of }}}^{n-1} \alpha^{2^{j}}+\sum^{2^{l}},
\end{aligned}
$$

where $0 \leq k \leq n-1$. Now the reader can check that the result is obtained by substituting $\alpha$ with $a^{-1}$ and computing the three cases of $b_{k}$. 


\section{Chapter 4}

\section{Compositional inverses and}

\section{complete mappings over finite}

\section{fields}

In this chapter ${ }^{1}$ we focus on CPPs and their compositional inverses. Let us recall we say that a permutation polynomial $f(x)$ is a complete permutation polynomial if $f(x)+x$ is also a permutation polynomial.

Using Proposition 2.1.1, Wu computed in [81] the compositional inverses, in explicit form, of arbitrary linearized permutation binomials over finite fields. We call a linearized polynomial a binomial if it has exactly two non-zero coefficients. As we showed in Chapter 3, the problem of computing the compositional inverses of certain classes of permutations is equivalent to obtaining the inverses of two other polynomials bijecting subspaces of the finite field, where one of these two is a linearized

\footnotetext{
${ }^{1}$ This chapter is substantively based on the following article originally published in Discrete Applied Mathematics, https://doi.org/10.1016/j.dam.2016.09.009. It is released under a Creative Commons Attribution Non-Commercial No Derivatives License.

[71] A. Tuxanidy and Q. Wang, Compositional inverses and complete mappings over finite fields, Discrete Applied Mathematics, v.217 no.2 (2017), p.318-329. https://doi.org/10.1016/j.dam. 2016.09 .009
} 
polynomial inducing a bijection between kernels of other linearized polynomials. To this end, we showed in Theorem 2.1.5 how to obtain linearized polynomials inducing the inverse map over subspaces on which a linearized polynomial induces a bijection. This in fact amounts to solving a system of linear equations. Thus, in particular, it is of interest to obtain explicit compositional inverses of linearized permutations of subspaces.

We start off in Section 4.1 by determining a class of linearized binomials permuting the kernel of the trace map and proceed to obtain, in Theorem 4.1.4, its inverse on the kernel. As an application of Theorem 4.1.4, we derive, in Theorem 4.3 .2 of Section 4.3, the compositional inverse of the complete permutation class in Theorem 4.2.3 generalizing some of the classes recently studied in [47, 63, 84-86]. Note that since inverses of complete mappings are also complete mappings, Theorem 4.3.2 and Corollary 4.3.3 imply the construction of a new, if rather complicated, class of complete permutation polynomials.

The reader will also find in Theorem 4.2.6 a recursive construction of complete mappings involving multi-trace functions. In addition we employ the CPP class of Theorem 4.2.3 to construct a set of mutually orthogonal complete mappings (see Corollary 4.2.8). Recall that we say two mappings $f, g$, of $\mathbb{F}_{q}$, are orthogonal if $f-g$ permutes $\mathbb{F}_{q}$. Note in particular that from such a set one may also readily obtain a set of mutually orthogonal Latin squares, an object of special interest in the literature (see for example [29]). 


\subsection{Inverses of linearized binomials permuting ker- nels of traces}

In this section we study the compositional inverses of binomials permuting the kernel of the trace map. More precisely, given a positive integer $r<n$, consider the binomial $L_{c, r}(x):=x^{p^{r}}-c x \in \mathbb{F}_{q^{n}}[x]$, where $c \in \mathbb{F}_{q}$. Note that $L_{c, r}\left(\operatorname{ker}\left(T_{q^{n} \mid q}\right)\right) \subseteq \operatorname{ker}\left(T_{q^{n} \mid q}\right)$, where $\operatorname{ker}\left(T_{q^{n} \mid q}\right)=\left\{\beta^{q}-\beta \mid \beta \in \mathbb{F}_{q^{n}}\right\}$ is the kernel of the additive map of $T_{q^{n} \mid q}$ on $\mathbb{F}_{q^{n}}$. We would like to discover what are the necessary and sufficient conditions for $L_{c, r}$ to be a permutation of $\operatorname{ker}\left(T_{q^{n} \mid q}\right)$, and in such cases obtain a polynomial in $\mathbb{F}_{q^{n}}[x]$ inducing the inverse map of $\left.L_{c, r}\right|_{\operatorname{ker}\left(T_{q^{n} \mid q}\right)}$. We only need to consider the case when $L_{c, r}$ permutes $\mathbb{F}_{q^{n}}$ and the case when $L_{c, r}$ permutes $\operatorname{ker}\left(T_{q^{n} \mid q}\right)$ but not $\mathbb{F}_{q^{n}}$. The former case has already been tackled in [81] (see Theorem 4.1.1 here) and so we focus on the latter case. We give the result in Theorem 4.1.6 of Subsection 4.1.1. Then in Subsection 4.1.2 we explain the method used to obtain the result. We remark that in Corollary 4.1.10 we show that under some restrictions of the characteristic, $p$, and the extension degree, $n, L_{c, r}$ permutes $\operatorname{ker}\left(T_{q^{n} \mid q}\right)$ for each $c \in \mathbb{F}_{q}$. Therefore it is quite useful to use this result to construct some permutation polynomials which are also complete mappings. Indeed, we demonstrate the applicability of Corollary 4.1.10 in the proof of Theorem 4.2.3 in Section 4.2.

\subsubsection{Statement and proof of result}

The following result due to $\mathrm{Wu}$ gives the compositional inverses of linearized permutation binomials $x^{q^{r}}-c x$ where $c$ lies in the extension $\mathbb{F}_{q^{n}}$. In the case when $c$ lies in $\mathbb{F}_{q}$, the binomial is a permutation of $\operatorname{ker}\left(T_{q^{n} \mid q}\right)$ and thus its inverse map over the kernel is clearly the restriction of the inverse over $\mathbb{F}_{q^{n}}$ to $\operatorname{ker}\left(T_{q^{n} \mid q}\right)$. We state this in Corollary 4.1.2. This accounts for the case when the binomial permuting the kernel 
of the trace has full rank. Later in Theorem 4.1.4 and 4.1.6 we tackle the case when the linearized binomial permutes the kernel of the trace map but not $\mathbb{F}_{q^{n}}$. Denote by $N_{q^{n} \mid q}: \mathbb{F}_{q^{n}} \rightarrow \mathbb{F}_{q}$ the norm function given by $N_{q^{n} \mid q}(x)=x^{\left(q^{n}-1\right) /(q-1)}$.

Theorem 4.1.1 (Theorem 2.1, [81]). Let $c \in \mathbb{F}_{q^{n}}^{*}$ and let $d:=(n, r)$. Then $L_{c, r}(x):=x^{q^{r}}-c x \in \mathbb{F}_{q^{n}}[x]$ permutes $\mathbb{F}_{q^{n}}$ if and only if $N_{q^{n} \mid q^{d}}(c) \neq 1$, in which case the compositional inverse of $L_{c, r}$ over $\mathbb{F}_{q^{n}}$ is given by

$$
L_{c, r}^{-1}(x)=\frac{N_{q^{n} \mid q^{d}}(c)}{1-N_{q^{n} \mid q^{d}}(c)} \sum_{i=0}^{\frac{n}{d}-1} c^{-\frac{q^{(i+1) r}-1}{q^{r}-1}} x^{q^{i r}} .
$$

Corollary 4.1.2. Let $q=p^{m}$ be a power of a prime number $p$, let $n, r$, be positive integers and denote $d:=(n m, r)$. If $c \in \mathbb{F}_{q}$ satisfies $N_{q^{n} \mid p^{d}}(c) \neq 1$, then $L_{c, r}(x):=$ $x^{p^{r}}-c x \in \mathbb{F}_{q^{n}}[x]$ permutes $\operatorname{ker}\left(T_{q^{n} \mid q}\right)$, having a compositional inverse over $\operatorname{ker}\left(T_{q^{n} \mid q}\right)$ given by

$$
L_{c, r}^{-1}(x)=\frac{N_{q^{n} \mid p^{d}}(c)}{1-N_{q^{n} \mid p^{d}}(c)} \sum_{i=0}^{\frac{n m}{d}-1} c^{-\frac{p^{(i+1) r}-1}{p^{r}-1}} x^{p^{i r}}
$$

Proof. Substituting $q$ and $n$ with $p$ and $n m$, respectively, in Theorem 4.1.1, we obtain that $L_{c, r}$ permutes $\mathbb{F}_{q^{n}}$. But since $L_{c, r}\left(\operatorname{ker}\left(T_{q^{n} \mid q}\right)\right) \subseteq \operatorname{ker}\left(T_{q^{n} \mid q}\right) \subseteq \mathbb{F}_{q^{n}}$, it follows that $L_{c, r}$ permutes $\operatorname{ker}\left(T_{q^{n} \mid q}\right)$. It now suffices to pick $L_{c, r}^{-1}$ as a compositional inverse of $L_{c, r}$ over $\operatorname{ker}\left(T_{q^{n} \mid q}\right)$, which is obtained from Theorem 4.1.1 through the aforementioned substitutions.

We now focus on the case when the linearized binomial permutes the kernel of the trace map but does not permute $\mathbb{F}_{q^{n}}$. We need the following lemma.

Lemma 4.1.3. Let $n, r, s$, be positive integers such that $s \mid n$ and $d:=(n, r)=(s, r)$. Then the following two identities hold.

(i) $T_{q^{n} \mid q^{s}}=\left.T_{q^{n r / d}\left|q^{s r / d}\right|}\right|_{\mathbb{q}^{n}} ;$

(ii) $N_{q^{s} \mid q^{d}}=\left.N_{q^{s r / d} \mid q^{r}}\right|_{\mathbb{F}_{q^{s}}}$. 
Proof. (i) Since $(n / d, r / d)=1$ and $n / s \mid n / d$ (implying $(n / s, r / d)=1)$, we have $\{k$ $(\bmod n / s) \mid 0 \leq k \leq n / s-1\}=\{k r / d(\bmod n / s) \mid 0 \leq k \leq n / s-1\}$ from which it follows that $\{k s(\bmod n) \mid 0 \leq k \leq n / s-1\}=\{k s r / d(\bmod n) \mid 0 \leq k \leq n / s-1\}$. Hence, for any $\alpha \in \mathbb{F}_{q^{n}}$, we get

$$
T_{q^{n} \mid q^{s}}(\alpha):=\sum_{k=0}^{\frac{n}{s}-1} \alpha^{q^{k s}}=\sum_{k=0}^{\frac{n}{s}-1} \alpha^{q^{k s r / d}}=: T_{q^{n r / d} \mid q^{s r / d}}(\alpha)
$$

as required.

(ii) Since $(s / d, r / d)=1$, we have $\{k(\bmod s / d) \mid 0 \leq k \leq s / d-1\}=\{k r / d$ $(\bmod s / d) \mid 0 \leq k \leq s / d-1\}$, implying $\{k d(\bmod s) \mid 0 \leq k \leq s / d-1\}=\{k r$ $(\bmod s) \mid 0 \leq k \leq s / d-1\}$. Thus, for any $\beta \in \mathbb{F}_{q^{s}}$, we obtain

$$
N_{q^{s} \mid q^{d}}(\beta):=\beta^{\sum_{k=0}^{\frac{s}{d}-1} q^{k d}}=\beta^{\sum_{k=0}^{\frac{s}{d}-1} q^{k r}}=: N_{q^{s r / d} \mid q^{r}}(\beta)
$$

Theorem 4.1.4. Let $q=p^{m}$ be a power of a prime number $p$, let $n, r, s$, be positive integers such that $s \mid n$ and $d:=(n, r)=(s, r)$, and let $c \in \mathbb{F}_{q^{s}}$ such that $N_{q^{s} \mid q^{d}}(c)=1$. Then the binomial $L_{c, r}(x):=x^{q^{r}}-c x \in \mathbb{F}_{q^{n}}[x]$ induces a permutation of $\operatorname{ker}\left(T_{q^{n} \mid q^{s}}\right)$ if and only if $p \nmid n / s$. In this case the compositional inverse of $L_{c, r}$ over $\operatorname{ker}\left(T_{q^{n}} \mid q^{s}\right)$ is given by

$$
\left.L_{c, r}\right|_{\operatorname{ker}\left(T_{q^{n} \mid q^{s}}\right)} ^{-1}(x)=\sum_{j=0}^{\frac{s}{d}-1} c^{-\frac{q^{(j+1) r}-1}{q^{r}-1}}\left(\left(\frac{n}{s}\right)^{-1} \sum_{k=1}^{\frac{n}{s}-1} k x^{q^{\frac{k s r}{d}}}\right)^{q^{j r}}
$$

Proof. Assume that $L_{c, r}$ does not permute $\mathbb{F}_{q^{s}}$, i.e., $N_{q^{s} \mid q^{d}}(c)=1$. Now suppose on the contrary that $p \mid n / s$. Then for any $k \in \mathbb{F}_{q^{s}}$, we have $T_{q^{n} \mid q^{s}}(k)=k n / s=0$; hence $\mathbb{F}_{q^{s}} \subseteq \operatorname{ker}\left(T_{q^{n} \mid q^{s}}\right)$. Then noting $L_{c, r}\left(\mathbb{F}_{q^{s}}\right) \subseteq \mathbb{F}_{q^{s}}$, we obtain that $L_{c, r}$ permutes $\mathbb{F}_{q^{s}}$, a contradiction. Necessarily, if $N_{q^{s} \mid q^{d}}(c)=1$ and $L_{c, r}$ permutes $\operatorname{ker}\left(T_{q^{n} \mid q^{s}}\right)$, 
then $p \nmid n / s$. To show that these are also sufficient conditions for $L_{c, r}$ to permute $\operatorname{ker}\left(T_{q^{n} \mid q^{s}}\right)$, it suffices to prove that $\left.L_{c, r}\right|_{\operatorname{ker}\left(T_{q^{n} \mid q^{s}}\right)} ^{-1}$, given above, induces the inverse of $\left.L_{c, r}\right|_{\operatorname{ker}\left(T_{q^{n} \mid q^{s}}\right)}$. First observe that $N_{q^{s} \mid q^{d}}(c)^{-q^{r}}=N_{q^{s} \mid q^{d}}(c)$ since $d \mid r$. Moreover $c^{\left(q^{s r / d}-1\right) /\left(q^{r}-1\right)}=N_{q^{s} \mid q^{d}}(c)=1$ by Lemma 4.1.3. For the sake of brevity denote $R(x):=(n / s)^{-1} \sum_{k=1}^{\frac{n}{s}-1} k x^{q^{k s r} / d}$. Now, for all $x \in \operatorname{ker}\left(T_{q^{n} \mid q^{s}}\right)$, we have

$$
\begin{aligned}
& L_{c, r}\left(\left.L_{c, r}\right|_{\operatorname{ker}\left(T_{q^{n} \mid q^{s}}\right)} ^{-1}(x)\right)=\left.L_{c, r}\right|_{\operatorname{ker}\left(T_{\left.q^{n} \mid q^{s}\right)}\right.} ^{-1}(x)^{q^{r}}-\left.c L_{c, r}\right|_{\operatorname{ker}\left(T_{q^{n} \mid q^{s}}\right)} ^{-1}(x) \\
& =\sum_{j=0}^{\frac{s}{d}-1}\left(c^{-q^{r}\left(\frac{q^{(j+1) r}-1}{q^{r}-1}\right)} R(x)^{q^{(j+1) r}}-c^{-\frac{q^{(j+1) r}-1}{q^{r}-1}+1} R(x)^{q^{j r}}\right) \\
& =\sum_{j=1}^{\frac{s}{d}} c^{-q^{r}\left(\frac{q^{j r}-1}{q^{r}-1}\right)} R(x)^{q^{j r}}-\sum_{j=0}^{\frac{s}{d}-1} c^{-q^{r}\left(\frac{q^{j r}-1}{q^{r}-1}\right)} R(x)^{q^{j r}} \\
& =c^{-q^{r}\left(\frac{q^{\frac{s}{d} r}-1}{q^{r}-1}\right)} R(x)^{q^{\frac{s}{d} r}}-R(x) \\
& =R(x)^{q^{\frac{s}{d} r}}-R(x) \\
& =\left(\frac{n}{s}\right)^{-1} \sum_{k=1}^{\frac{n}{s}-1} k\left(x^{q^{(k+1) \frac{s r}{d}}}-x^{q^{k \frac{s r}{d}}}\right) \\
& =\left(\frac{n}{s}\right)^{-1}\left(\sum_{k=2}^{\frac{n}{s}}(k-1) x^{q^{k \frac{s r}{d}}}-\sum_{k=1}^{\frac{n}{s}-1} k x^{q^{k \frac{s r}{d}}}\right) \\
& =\left(\frac{n}{s}\right)^{-1}\left(\left(\frac{n}{s}-1\right) x-\sum_{k=1}^{\frac{n}{s}-1} x^{q^{k \frac{s r}{d}}}\right) \\
& =x-\left(\frac{n}{s}\right)^{-1} T_{q^{n r / d} \mid q^{s r / d}}(x) \\
& =x-\left(\frac{n}{s}\right)^{-1} T_{q^{n} \mid q^{s}}(x) \\
& =x
\end{aligned}
$$

as required.

Remark 4.1.5. In his PhD thesis $W u$ [82] had already tackled the case when $(n, r)=1$ 
(see Theorem 3.4.6 there) although the result there was obtained through trial and error. In Subsection 4.1.2 we show the methodology from which the more general Theorem 4.1.4 can be obtained. It employs recent techniques from both [81] and [70].

By the means of some substitutions we obtain the following equivalent result.

Theorem 4.1.6. Let $q=p^{m}$ be a power of a prime number $p$, let $n, r$, be positive integers such that $d:=(n m, r)=(m, r)$, and let $c \in \mathbb{F}_{q}$ such that $N_{q \mid p^{d}}(c)=1$. Then $L_{c, r}(x):=x^{p^{r}}-c x$ induces a permutation of $\operatorname{ker}\left(T_{q^{n} \mid q}\right)$ if and only if $p \nmid n$. In this case the compositional inverse of $L_{c, r}$ over $\operatorname{ker}\left(T_{q^{n} \mid q}\right)$ is given by

$$
\left.L_{c, r}\right|_{\operatorname{ker}\left(T_{q^{n} \mid q}\right)} ^{-1}(x)=\sum_{j=0}^{\frac{m}{d}-1} c^{-\frac{p^{(j+1) r}-1}{p^{r}-1}}\left(n^{-1} \sum_{k=1}^{n-1} k x^{p^{\frac{k m r}{d}}}\right)^{p^{j r}}
$$

Proof. The result follows from Theorem 4.1.4 if we substitute $q, s, n$, there with $p, m, n m$, respectively.

Remark 4.1.7. Theorem 4.1.4 and 4.1.6 are equivalent. Indeed, given $x^{q^{r}}-c x \in$ $\mathbb{F}_{q^{n}}[x]$ satisfying Theorem 4.1.4, i.e., $c \in \mathbb{F}_{q^{s}}$ with $s \mid n,(s, r)=(n, r)$ and $q=p^{m}$, we get $x^{q^{r}}-c x=x^{p^{m r}}-c x \in \mathbb{F}_{q_{1}^{n / s}}[x]$ where $c \in \mathbb{F}_{q_{1}}$ with $q_{1}:=q^{s}=p^{m s}, T_{q^{n} \mid q^{s}}=T_{q_{1}^{n / s} \mid q_{1}}$, and $(m s \cdot n / s, m r)=(m n, m r)=(m s, m r)$, satisfying Theorem 4.1.6. Now the fact that Theorem 4.1.6 follows from Theorem 4.1 .4 implies the equivalence of the two.

Corollary 4.1.8 (Lemma 3.4, [84]). Let $q=p^{m}$ be a power of a prime number $p$, let $n, r$, be positive integers such that $(n, r)=1$, and let $c \in \mathbb{F}_{q}$. Then $x^{p^{r}}-c x$ permutes $\operatorname{ker}\left(T_{q^{n} \mid q}\right)$ if and only if c belongs to any of the following two cases.

(i) $N_{q \mid p^{(m, r)}}(c)=1$ and $p \nmid n$;

(ii) $N_{q^{n} \mid p^{(m, r)}}(c) \neq 1$.

Proof. Using the fact that $d:=(n m, r)=(m, r)$ because $(n, r)=1$ by assumption, the result (i) follows from Theorem 4.1.6, while (ii) follows from Corollary 4.1.2. 
Remark 4.1.9. By choosing, say $n=8$ and $m=r=2$, it is easy to see that the hypotheses of Theorem 4.1.6 are in fact more general than those of Corollary 4.1.8.

The following corollary shows that under some restrictions on $p$ and $n, L_{c, r}$ permutes $\operatorname{ker}\left(T_{q^{n} \mid q}\right)$ for each $c \in \mathbb{F}_{q}$. Later on, in Section 3, we will make use of this result in order to construct a class of complete mappings.

Corollary 4.1.10. Let $q=p^{m}$ be a power of a prime number $p$, let $n, r$, be positive integers such that $d:=(n m, r)=(m, r), p \nmid n$, and $\left(n, p^{d}-1\right)=1$. Then $L_{c, r}(x):=$ $x^{p^{r}}-c x$ induces a permutation of $\operatorname{ker}\left(T_{q^{n} \mid q}\right)$ for each $c \in \mathbb{F}_{q}$.

Proof. Clearly, if $c=0$, then $L_{c, r}$ permutes $\operatorname{ker}\left(T_{q^{n} \mid q}\right)$. We may thus assume that $c \in \mathbb{F}_{q}^{*}$. If $N_{q \mid p^{d}}(c)=1$, then $L_{c, r}$ permutes $\operatorname{ker}\left(T_{q^{n} \mid q}\right)$ by Theorem 4.1 .6 (because $p \nmid n$ additionally, by assumption). Now assume $N_{q \mid p^{d}}(c) \neq 1$. We claim that $N_{q \mid p^{d}}(c)^{n} \neq 1$; equivalently, since $N_{q^{n} \mid p^{d}}(c)=N_{q \mid p^{d}} \circ N_{q^{n} \mid q}(c)=N_{q \mid p^{d}}(c)^{n} \neq 1, L_{c, r}$ permutes $\mathbb{F}_{q^{n}}$ and hence $\operatorname{ker}\left(T_{q^{n} \mid q}\right)$ by Corollary 4.1.2. Denote by $t$ the multiplicative order of $N_{q \mid p^{d}}(c) \in \mathbb{F}_{p^{d}}^{*}$. It is clear that $t \mid\left(p^{d}-1\right)$. On the contrary suppose that $N_{q \mid p^{d}}(c)^{n}=1$.

Then $t \mid n$ as well. As a result, $t \mid\left(n, p^{d}-1\right)=1$ giving $t=1$, a contradiction to our assumption $N_{q \mid p^{d}}(c) \neq 1$. It follows that $L_{c, r}$ permutes $\operatorname{ker}\left(T_{q^{n} \mid q}\right)$ for all $c \in \mathbb{F}_{q}$.

\subsubsection{Method used to obtain the inverse in Theorem 4.1.4}

We know from Theorem 4.1.1 that $L_{c, r}(x)=x^{q^{r}}-c x$ is a permutation polynomial over $\mathbb{F}_{q^{n}}$ if and only if $N_{q^{n} \mid q^{d}}(c) \neq 1$, where $d=(n, r)$. In this case $L_{c, r}$ must also permute $\operatorname{ker}\left(T_{q^{n} \mid q^{s}}\right)$ if $c \in \mathbb{F}_{q}$ and thus we may take $L_{c, r}^{-1}$ to be the compositional inverse over $\operatorname{ker}\left(T_{q^{n} \mid q^{s}}\right)$, as done in Corollary 4.1.2. In this subsection we consider the case when $L_{c, r}$ permutes $\operatorname{ker}\left(T_{q^{n}} \mid q^{s}\right)$ but does not permute $\mathbb{F}_{q^{n}}$ (Theorem 4.1.4), and attempt to obtain a compositional inverse over $\operatorname{ker}\left(T_{q^{n}} \mid q^{s}\right)$. Some of the arguments bear similarity to those employed in [81] where the compositional inverse of linearized 
binomials with full rank was obtained. It consists of modifying the initial problem into an easier one via substitutions of the parameters $q, n$, and in our case, $s$ as well, and then computing the inverse over a convenient superspace of $\operatorname{ker}\left(T_{q^{n}} \mid q^{s}\right)$.

If we let $q_{1}:=q^{d}$, where $d:=(n, r)=(s, r)$, then $L_{c, r}(x)$ becomes $x^{q_{1}^{r / d}}-c x \in$ $\mathbb{F}_{q^{n}}[x]=\mathbb{F}_{q_{1}^{n / d}}[x]$ with $c \in \mathbb{F}_{q^{s}}=\mathbb{F}_{q_{1}^{s / d}}, T_{q^{n} \mid q^{s}}=T_{q_{1}^{n / d} \mid q_{1}^{s / d}}, N_{q^{n} \mid q^{d}}(c)=N_{q_{1}^{n / d} \mid q_{1}^{s / d}}(c)=1$, and $(n / d, r / d)=(s / d, r / d)=1$. Thus we first consider the case when $(n, r)=(s, r)=$ 1. Now view $L_{c, r}$ as a polynomial over the composite field $\mathbb{F}_{q^{n r}}$ and observe that $L_{c, r} \in$ $\mathscr{L}_{n}\left(\mathbb{F}_{q^{n r}}\right)$, i.e., $L_{c, r}$ is a $q^{r}$-polynomial over $\mathbb{F}_{q^{n r}}$. From Lemma 4.1 .3 we know that $\left.T_{q^{n r} \mid q^{s r}}\right|_{\mathbb{F}^{n}}=T_{q^{n} \mid q^{s}}$ and thus $\operatorname{ker}\left(T_{q^{n} \mid q^{s}}\right)=\operatorname{ker}\left(\left.T_{q^{n r} \mid q^{s r}}\right|_{\mathbb{F}^{n}}\right) \subseteq \operatorname{ker}\left(T_{q^{n r} \mid q^{s r}}\right)$. Moreover $L_{c, r}\left(\operatorname{ker}\left(T_{q^{n r}} \mid q^{s r}\right)\right) \subseteq \operatorname{ker}\left(T_{q^{n r} \mid q^{s r}}\right)$. It follows that if $L_{c, r}$ permutes $\operatorname{ker}\left(T_{q^{n r}} \mid q^{s r}\right)$, then the inverse map of $\left.L_{c, r}\right|_{\operatorname{ker}\left(T_{q^{n}} q^{s}\right)}$ can be obtained from the inverse of $\left.L_{c, r}\right|_{\operatorname{ker}\left(T_{q^{n r}} \mid q^{s r}\right)}$ by restricting its domain to $\mathbb{F}_{q^{n}}$. Now in order to obtain the inverse of $L_{c, r}$ over $\operatorname{ker}\left(T_{q^{n r} \mid q^{s r}}\right)$, we make use of Theorem 2.1.5.

Since $\operatorname{ker}\left(T_{q^{n r} \mid q^{s r}}\right)$ is an $\mathbb{F}_{q^{r}}$-subspace of $\mathbb{F}_{q^{n r}}$, and $L_{c, r} \in \mathscr{L}_{n}\left(\mathbb{F}_{q^{n r}}\right)$ is a $q^{r}$-polynomial over $\mathbb{F}_{q^{n r}}$ inducing (by assumption) a permutation of $\operatorname{ker}\left(T_{q^{n r}} q^{s r}\right)$, Theorem 2.1.5 applies. Noting that $(n / s)^{-1} T_{q^{n r} \mid q^{s r}} \in \mathscr{L}_{n}\left(\mathbb{F}_{q^{n r}}\right)$ is idempotent on $\mathbb{F}_{q^{n r}}$ having kernel $\operatorname{ker}\left(T_{q^{n r} \mid q^{s r}}\right)$, we can deduce from Theorem 2.1.5 that $L_{c, r}$ permutes $\operatorname{ker}\left(T_{q^{n r}} \mid q^{s r}\right)$ (hence $\left.\operatorname{ker}\left(T_{q^{n} \mid q^{s}}\right)\right)$ if and only if there exists a solution $\bar{d}=\left(d_{0}, \ldots, d_{n-1}\right)$ to the linear equation

$$
\begin{aligned}
\bar{d} D_{L_{c, r}} & =v_{q^{r}, n}\left(x-\left(\frac{n}{s}\right)^{-1} T_{q^{n r} \mid q^{s r}}(x)\right) \\
& =-\left(\frac{n}{s}\right)^{-1}\left(1-\frac{n}{s}, 0,0, \ldots, 0,1,0,0, \ldots, 0,1,0,0, \ldots\right),
\end{aligned}
$$

where the non-zero entries on the right hand side occur at indices (which start at 0 
and end at $n-1)$ given by $k s$ with $0 \leq k<n / s$, and

$$
D_{L_{c, r}}=\left(\begin{array}{cccccc}
-c & 1 & 0 & 0 & \cdots & 0 \\
0 & -c^{q^{r}} & 1 & 0 & \cdots & 0 \\
\vdots & \vdots & \vdots & \vdots & & \vdots \\
1 & 0 & 0 & 0 & \cdots & -c^{q^{(n-1) r}}
\end{array}\right)
$$

is the associate Dickson matrix of $L_{c, r} \in \mathscr{L}_{n}\left(\mathbb{F}_{q^{n r}}\right)$. If so, it follows that the polynomial $\sum_{i=0}^{n-1} d_{i} x^{q^{i r}}$ induces the inverse map of $\left.L_{c, r}\right|_{\operatorname{ker}\left(T_{q} n r \mid q^{s r}\right)}$, and hence of $\left.L_{c, r}\right|_{\operatorname{ker}\left(T_{q^{n} \mid q^{s}}\right)}$. Solving the linear equation we obtain a solution

$$
d_{k s+j}=\left(\frac{n}{s}\right)^{-1} k c^{-\frac{q^{(j+1) r}-1}{q^{r}-1}}
$$

where $0 \leq j<s$ and $0 \leq k<n / s$. To see this, it suffices to show that $\bar{d}$ satisfies the linear equation, i.e., that the $(k s+j)$-th entry, $\left(\bar{d} D_{L_{c, r}}\right)_{k s+j}$, of the $n$-tuple $\bar{d} D_{L_{c, r}}$, satisfies

$$
\left(\bar{d} D_{L_{c, r}}\right)_{k s+j}= \begin{cases}1-\left(\frac{n}{s}\right)^{-1} & \text { if } j=k=0 \\ -\left(\frac{n}{s}\right)^{-1} & \text { if } j=0 \text { and } k \geq 1 \\ 0 & \text { otherwise, }\end{cases}
$$

where $0 \leq j<s$ and $0 \leq k<n / s$. First recall that we have made the assumptions that $N_{q^{s} \mid q}\left(c^{-1}\right)=1(d=1$ since $d:=(n, r)=(s, r)=1$ by our assumption above $)$ 
and $p \nmid n / s$ of Theorem 4.1.4. Then Lemma 4.1.3 gives $N_{q^{s r} \mid q^{r}}\left(c^{-1}\right)=1$. We have

$$
\begin{aligned}
\left(\bar{d} D_{L_{c, r}}\right)_{0} & =-d_{0} c+d_{n-1}=0+d_{\left(\frac{n}{s}-1\right) s+s-1} \\
& =\left(\frac{n}{s}\right)^{-1}\left(\frac{n}{s}-1\right) N_{q^{s r} \mid q^{r}}\left(c^{-1}\right) \\
& =1-\left(\frac{n}{s}\right)^{-1} ; \\
\left(\bar{d} D_{L_{c, r}}\right)_{0<k<n / s}^{k s} & =-d_{k s} c^{q^{k s r}}+d_{k s-1}=-d_{k s} c+d_{(k-1) s+s-1} \\
& =\left(\frac{n}{s}\right)^{-1}\left[-k c^{-1} c+(k-1) N_{q^{s r} \mid q^{r}}\left(c^{-1}\right)\right] \\
& =-\left(\frac{n}{s}\right)^{-1} ; \text { and } \\
\left(\bar{d} D_{L_{c, r}}\right)_{\substack{k s+j \\
0<j<s}} & -d_{k s+j} c^{q^{(k s+j) r}}+d_{k s+j-1} \\
& =\left(\frac{n}{s}\right)^{-1} k\left[-c^{-\frac{q^{(j+1) r}-1}{q^{r}-1}} c^{q^{j r}}+c^{-\frac{q^{j r}-1}{q^{r}-1}}\right] \\
& =\left(\frac{n}{s}\right)^{-1} k\left[-c^{-\frac{q^{j r}-1}{q^{r}-1}}+c^{-\frac{q^{j r}-1}{q^{r}-1}}\right] \\
& =0,
\end{aligned}
$$

as required. As a result, if $(n, r)=1$, then $N_{q^{s} \mid q}(c)=1$ and $p \nmid n / s$ are sufficient conditions for $L_{c, r}$ to permute $\operatorname{ker}\left(T_{q^{n} \mid q^{s}}\right)$ but not $\mathbb{F}_{q^{n}}$. In this case, one of the polynomials inducing the inverse map of $\left.L_{c, r}\right|_{\operatorname{ker}\left(T_{q^{n} \mid q^{s}}\right)}$ is given by

$$
\begin{aligned}
\left.L_{c, r}\right|_{\operatorname{ker}\left(T_{q^{n} \mid q^{s}}\right.} ^{-1}(x) & =\left(\frac{n}{s}\right)^{-1} \sum_{j=0}^{s-1} \sum_{k=1}^{\frac{n}{s}-1} k c^{-\frac{q^{(j+1) r}-1}{q^{r}-1}} x^{q^{(k s+j) r}} \\
& =\sum_{j=0}^{s-1} c^{-\frac{q^{(j+1) r}-1}{q^{r}-1}}\left(\left(\frac{n}{s}\right)^{-1} \sum_{k=1}^{\frac{n}{s}-1} k x^{q^{k s r}}\right)^{q^{j r}} .
\end{aligned}
$$

Now for the general case of $1 \leq r \leq n-1$ and $d=(n, r)=(s, r)$, substitute $q, n, r, s$, with $q^{d}, n / d, r / d, s / d$, respectively, in the expression above for $\left.L_{c, r}\right|_{\operatorname{ker}\left(T_{q^{n} \mid q^{s}}\right)} ^{-1}$, to obtain the result in Theorem 4.1.4. 


\subsection{Extensions of a class of complete permutation polynomials}

In this section we improve upon a result due to $\mathrm{Wu}$ and Lin (Theorem 3.7 in [84]). See Theorem 4.2.3 below yielding Corollary 4.2.5. Then in Theorem 4.2.6 we give a recursive construction of complete mappings involving multi-trace functions.

First we need the following lemma due to Coulter-Henderson-Mathews, a consequence of the AGW criterion [3].

Lemma 4.2.1 (Theorem 3, [24]). Let $q=p^{m}$ be a prime power, let $g \in \mathbb{F}_{q}[x]$, let $H \in \mathbb{F}_{q}[x]$ be a p-polynomial, and let $f(x)=H(x)+x g\left(T_{q^{n} \mid q}(x)\right)$. Then $f(x)$ is a permutation polynomial over $\mathbb{F}_{q^{n}}$ if and only if the following two conditions hold.

(i) $\varphi_{y}(x):=H(x)+x g(y)$ induces a permutation of $\operatorname{ker}\left(T_{q^{n} \mid q}\right)=\left\{\beta^{q}-\beta \mid \beta \in \mathbb{F}_{q^{n}}\right\}$ for each $y \in \mathbb{F}_{q}$.

(ii) $\bar{f}(x):=H(x)+x g(x)$ induces a permutation of $\mathbb{F}_{q}$.

The following consequence is straightforward.

Corollary 4.2.2. Let $q=p^{m}$ be a prime power and let $g \in \mathbb{F}_{q}[x]$. Then $x g\left(T_{q^{n} \mid q}(x)\right)$ is a permutation of $\mathbb{F}_{q^{n}}$ if and only if $x g(x)$ is a permutation of $\mathbb{F}_{q}$ and $g(0) \neq 0$.

The following represents an extension to Theorem 3.7 in [84], replacing the hypothesis of $(n, r)=1$ there with a more general case $(m n, r)=(m, r)$. For $f \in \mathbb{F}_{q}[x]$ inducing a permutation of a subspace $V$ of $\mathbb{F}_{q}$, we say that $f$ is a partial CPP over $V$ if $f(x)+x$ also induces a permutation of $V$.

Theorem 4.2.3. Let $q=p^{m}$ be a power of a prime number $p$, let $G \in \mathbb{F}_{q}[x]$, and let $n, r$, be positive integers such that $d:=(m, r)=(m n, r), p \nmid n$ and $\left(n, p^{(m, r)}-1\right)=1$. Then

$$
f(x)=a x^{p^{r}}+x\left(G\left(T_{q^{n} \mid q}(x)\right)-a T_{q^{n} \mid q}(x)^{p^{r}-1}\right)
$$


is a complete permutation polynomial over $\mathbb{F}_{q^{n}}$ for each $a \in \mathbb{F}_{q}^{*}$ if and only if $x G(x)$ is a complete permutation polynomial over $\mathbb{F}_{q}$.

Proof. Note that both $f(x)$ and $f(x)+x$ are instances of Lemma 4.2.1. It follows that $f$ is a complete permutation polynomial over $\mathbb{F}_{q^{n}}$ if and only if $\varphi_{y}(x):=a x^{p^{r}}+$ $x\left(G(y)-a y^{p^{r}-1}\right)=a\left[x^{p^{r}}+x\left(G(y)-a y^{p^{r}-1}\right) / a\right]$ is a partial complete permutation polynomial over $\operatorname{ker}\left(T_{q^{n} \mid q}\right)$, and $\bar{f}(x):=a x^{p^{r}}+x\left(G(x)-a x^{p^{r}-1}\right)=x G(x)$ is a complete permutation polynomial over $\mathbb{F}_{q}$. The former holds for each $a \in \mathbb{F}_{q}^{*}$ by Corollary 4.1.10, whereas the latter implies the result.

Theorem 4.2.3 generalizes [84, Theorem 3.7], [47, Theorem 3], [63, Theorem 4.1], [85, Theorem 2.1], and [86, Theorem 6].

Example 4.2.4. Let $p=7, m=1, n=5, r=2$. Let $G(x)=x^{3}+3$ and thus $x G(x)=x^{4}+3 x$ is a complete mapping over $\mathbb{F}_{7}$. Then

$$
f(x)=a x^{7^{2}-1}+x\left(\left(T_{7^{5} \mid 7}(x)\right)^{3}+3-a T_{7^{5} \mid 7}(x)^{7^{2}-1}\right)
$$

is a complete mapping over $\mathbb{F}_{7^{5}}$ for each $a \in \mathbb{F}_{7}^{*}$. For instance, when $a=2$, we obtain a complete mapping with degree 14407 and 247 nonzero terms, modulo $x^{7^{5}}-x$.

Letting $G=b \in \mathbb{F}_{q} \backslash\{-1,0\}$ be arbitrary in Theorem 4.2.3, the following corollary is straightforward.

Corollary 4.2.5. Let $q=p^{m}$ be a power of a prime number $p$, and let $n, r$, be positive integers such that $d:=(m, r)=(m n, r), p \nmid n$ and $\left(n, p^{(m, r)}-1\right)=1$. Then

$$
f(x)=a\left(x^{p^{r}}-x T_{q^{n} \mid q}(x)^{p^{r}-1}\right)+b x
$$

is a complete permutation polynomial over $\mathbb{F}_{q^{n}}$ for each $a \in \mathbb{F}_{q}$ and each $b \in \mathbb{F}_{q} \backslash$ $\{-1,0\}$. 
Finally we give a recursive construction of CPPs involving multi-trace functions.

Theorem 4.2.6. Let $q=p^{m}$ be a power of a prime number $p$, let $n, r$ and $1=$ : $d_{0}\left|d_{1}\right| \cdots \mid d_{t}:=n$ be positive integers such that $p \nmid n,\left(n, p^{(m, r)}-1\right)=1$ and $\left(d_{i} m, r\right)=\left(d_{j} m, r\right)$ for each $0 \leq i, j \leq t$. Let $a_{0}, \ldots, a_{t-1}$, be such that for $0 \leq k \leq t-1, a_{k} \in \mathbb{F}_{q^{d_{k}}}$ and $\sum_{l=0}^{k} a_{l} \neq 0$. Let $f_{0} \in \mathbb{F}_{q}[x]$. Then

$$
f(x)=x\left(\sum_{k=0}^{t-1} a_{k}\left(x^{p^{r}-1}-T_{q^{n} \mid q^{d_{k}}}(x)^{p^{r}-1}\right)+\frac{f_{0}\left(T_{q^{n} \mid q}(x)\right)}{T_{q^{n} \mid q}(x)}\right)
$$

is a complete permutation polynomial over $\mathbb{F}_{q^{n}}$ if and only if $f_{0}$ is a complete permutation polynomial over $\mathbb{F}_{q}$ satisfying $f_{0}(0)=0$.

Proof. For the sake of brevity denote $T_{j}^{k}:=T_{q^{d_{k} \mid q^{d_{j}}}}$ if $j \leq k$. For each $0 \leq i \leq t-1$, let $c_{i}:=\sum_{l=0}^{i} a_{l} \in \mathbb{F}_{q^{d_{i}}}^{*}$ and recursively define the polynomials

$$
f_{i+1}(x):=x\left(c_{i} x^{p^{r}-1}-c_{i} T_{i}^{i+1}(x)^{p^{r}-1}+\frac{f_{i}\left(T_{i}^{i+1}(x)\right)}{T_{i}^{i+1}(x)}\right) \in \mathbb{F}_{q^{d_{i+1}}}[x]
$$

If we substitute $G(x), m, n, q$, in Theorem 4.2.3, with $x^{q^{d_{i}-2}} f_{i}(x), d_{i} m, d_{i+1} / d_{i}, q^{d_{i}}$, respectively, then each $f_{i+1}$ satisfies the conditions of Theorem 4.2.3. Indeed, we have $\left(\frac{d_{i+1}}{d_{i}} \cdot d_{i} m, r\right)=\left(d_{i+1} m, r\right)=\left(d_{i} m, r\right)$ by assumption; $p \nmid d_{i+1} / d_{i}$ (because $\left.p \nmid n\right)$ and $\left(\frac{d_{i+1}}{d_{i}}, p^{\left(d_{i} m, r\right)}-1\right)=\left(d_{i+1} / d_{i}, p^{(m, r)}-1\right)=1$ since $\left(d_{i+1} / d_{i}\right) \mid n$ and $\left(n, p^{(m, r)}-1\right)=1$ by assumption as well. Then it follows from Theorem 4.2.3 that $f_{i+1}$ is a CPP over $\mathbb{F}_{q^{d_{i}\left(d_{i+1} / d_{i}\right)}}=\mathbb{F}_{q^{d_{i+1}}}$ if and only if $x^{q^{d_{i}}-1} f_{i}(x)$ is a CPP over $\mathbb{F}_{q^{d_{i}}}$. Note that

$$
\left.x^{q^{d_{i}-1}} f_{i}(x)\right|_{\mathbb{F}_{q^{d_{i}}}}=\left\{\begin{array}{l}
f_{i}(x), \text { if } i \geq 1\left(\text { since } f_{i}(0)=0 \text { for each } i \geq 1\right) \\
x^{q-1} f_{0}(x), \text { if } i=0 .
\end{array}\right.
$$


Denote $H(x):=x^{q-1} f_{0}(x)$. We claim that $f_{1}$ is a CPP over $\mathbb{F}_{q^{d_{1}}}$ if and only if $f_{0}$ is a CPP over $\mathbb{F}_{q}$ satisfying $f_{0}(0)=0$. Indeed, if $f_{0}(0)=0$, then $\left.H\right|_{\mathbb{F}_{q}}=f_{0}$ implying that $f_{1}$ is $\mathrm{CPP}$ over $\mathbb{F}_{q^{d_{1}}}$ if and only if $f_{0}$ is a $\mathrm{CPP}$ over $\mathbb{F}_{q}$. On the other hand, if $f_{0}(0) \neq 0$, write $f_{0}(x)=A(x)+b$ for some $A \in \mathbb{F}_{q}[x]$ such that $A(0)=0$ and some $b \in \mathbb{F}_{q}^{*}$. We need to show that $H$ is not a CPP over $\mathbb{F}_{q}$. On the contrary, suppose that $H$ is a $\mathrm{CPP}$ (and hence PP) over $\mathbb{F}_{q}$. Since $H(0)=0$, it follows that $H$ permutes $\mathbb{F}_{q}^{*}$. But $\left.H\right|_{\mathbb{F}_{q}^{*}}=\left.f_{0}\right|_{\mathbb{F}_{q}^{*}}$. Then $f_{0}$ permutes $\mathbb{F}_{q}^{*}$. This in turn implies that $A$ permutes $\mathbb{F}_{q}^{*}$. Hence $-b \in A\left(\mathbb{F}_{q}^{*}\right)$ giving $f_{0}(e)=0$ for some $e \in \mathbb{F}_{q}^{*}$. But then $H(e)=f_{0}(e)=0$, a contradiction. The claim follows. Now induction yields that for $1 \leq i \leq t, f_{i}$ is a CPP over $\mathbb{F}_{q^{d_{i}}}$ if and only if $f_{0}$ is a CPP over $\mathbb{F}_{q}$ satisfying $f_{0}(0)=0$. Next, assuming $f_{0}(0)=0$, we claim that for $0 \leq i \leq t$,

$$
f_{i}(x)=x\left(\sum_{k=0}^{i-1} a_{k}\left(x^{p^{r}-1}-T_{k}^{i}(x)^{p^{r}-1}\right)+\frac{f_{0}\left(T_{0}^{i}(x)\right)}{T_{0}^{i}(x)}\right) .
$$

Proceed by induction on $0 \leq i \leq t$. When $i=0$ the claim is clear. Assume (4.2.1) holds for some $i<n$. Then by the transitivity of the trace function, we get

$$
\frac{f_{i}\left(T_{i}^{i+1}(x)\right)}{T_{i}^{i+1}(x)}=\sum_{k=0}^{i-1} a_{k}\left(T_{i}^{i+1}(x)^{p^{r}-1}-T_{k}^{i+1}(x)^{p^{r}-1}\right)+\frac{f_{0}\left(T_{0}^{i+1}(x)\right)}{T_{0}^{i+1}(x)} .
$$


Thus, by the definition of $f_{i+1}$, we have

$$
\begin{aligned}
f_{i+1}(x)= & x\left(c_{i} x^{p^{r}-1}-c_{i} T_{i}^{i+1}(x)^{p^{r}-1}\right. \\
& \left.\quad+\sum_{k=0}^{i-1} a_{k}\left(T_{i}^{i+1}(x)^{p^{r}-1}-T_{k}^{i+1}(x)^{p^{r}-1}\right)+\frac{f_{0}\left(T_{0}^{i+1}(x)\right)}{T_{0}^{i+1}(x)}\right) \\
= & x\left(\sum_{k=0}^{i} a_{k} x^{p^{r}-1}-\sum_{k=0}^{i} a_{k} T_{i}^{i+1}(x)^{p^{r}-1}\right. \\
& \left.\quad+\sum_{k=0}^{i-1} a_{k}\left(T_{i}^{i+1}(x)^{p^{r}-1}-T_{k}^{i+1}(x)^{p^{r}-1}\right)+\frac{f_{0}\left(T_{0}^{i+1}(x)\right)}{T_{0}^{i+1}(x)}\right) \\
= & x\left(\sum_{k=0}^{i} a_{k} x^{p^{r}-1}-\sum_{k=0}^{i} a_{k} T_{k}^{i+1}(x)^{p^{r}-1}+\frac{f_{0}\left(T_{0}^{i+1}(x)\right)}{T_{0}^{i+1}(x)}\right) \\
= & x\left(\sum_{k=0}^{i} a_{k}\left(x^{p^{r}-1}-T_{k}^{i+1}(x)^{p^{r}-1}\right)+\frac{f_{0}\left(T_{0}^{i+1}(x)\right)}{T_{0}^{i+1}(x)}\right),
\end{aligned}
$$

satisfying the expression in (4.2.1). The claim follows. Now it only remains to notice that $f=f_{t}$.

Theorem 4.2.6 generalizes [63, Corollary 4.4] and [85, Corollary 2.3].

No even $n$ satisfies the conditions of Theorem 4.2.6, since $p$ has to be odd but then $\left(n, p^{(m, r)}-1\right) \neq 1$. Thus $n$ has to be odd. In this case, when $r=1$, the result works for any prime $p$ and odd $n$ such that $(n, p(p-1))=1$. Similarly, let $m=r, p$ be any prime and let $n$ such that $q=p^{m}$ and $\left(n, p\left(p^{m}-1\right)\right)=1$. For any complete mapping $f_{0}(x) \in \mathbb{F}_{q}[x]$ and $a_{i}$ 's satisfying the conditions in Theorem 4.2.6, we obtain complete mappings over $\mathbb{F}_{q^{n}}$ using multi-trace functions. In the following we give another example that does not belong to the above two cases.

Example 4.2.7. Let $p=5, n=9, m=1, r=2$. Let $d_{0}=1, d_{1}=3, d_{2}=9$. Choose $a_{0} \in \mathbb{F}_{5}^{*}$ and $a_{1} \in \mathbb{F}_{5^{3}}$ such that $a_{1}+a_{0} \neq 0$. It is easy to show that $f_{0}(x)=a x^{5}-x$ is a complete mapping over $\mathbb{F}_{5}$ for any $a \neq 1$. Now the conditions of Theorem 4.2.6 
are satisfied. Hence

$$
\begin{aligned}
f(x) & =x\left(a_{0}\left(x^{5^{2}-1}-T_{5^{9} \mid 5}(x)^{5^{2}-1}\right)+a_{1}\left(x^{5^{2}-1}-T_{5^{9} \mid 5^{3}}(x)^{5^{2}-1}\right)+a T_{5^{9} \mid 5}(x)^{4}-1\right) \\
& =\left(a_{0}+a_{1}\right) x^{5^{2}}-x\left(a_{0} T_{5^{9} \mid 5}(x)^{5^{2}-1}+a_{1} T_{5^{9} \mid 5^{3}}(x)^{5^{2}-1}-a T_{5^{9} \mid 5}(x)^{4}+1\right)
\end{aligned}
$$

is a complete mapping over $\mathbb{F}_{5^{9}}$.

The CPP class of Theorem 4.2.3 yields a set of orthogonal complete mappings. It is well-known that a set of $k$ pairwise orthogonal complete mappings of $\mathbb{F}_{q^{n}}$ yields a set of $k+1$ mutually orthogonal Latin squares (MOLS) [29]. See also [62,63] for details on MOLS and typical construction methods. We have the following consequence of Theorem 4.2.3.

Corollary 4.2.8. Let $q=p^{m}$ be a power of a prime number $p$ and let $n, r$, be positive integers such that $(m, r)=(m n, r), p \nmid n$ and $\left(n, p^{(m, r)}-1\right)=1$. Let $\left\{b_{i}\right\}$ be a subset of $\mathbb{F}_{q}$ and let $G \in \mathbb{F}_{q}[x]$ such that each $\bar{f}_{i}(x):=x\left(G(x)+b_{i}\right)$ is a complete permutation polynomial over $\mathbb{F}_{q}$. For each such $b_{i}$, let $a_{i} \in \mathbb{F}_{q}^{*}$. Then the mappings

$$
P_{i}(x)=x\left(a_{i} x^{p^{r}-1}-a_{i} T_{q^{n} \mid q}(x)^{p^{r}-1}+G\left(T_{q^{n} \mid q}(x)\right)+b_{i}\right), \quad i \geq 1,
$$

form a set of $\left|\left\{b_{i}\right\}\right|$ pairwise orthogonal complete mappings of order $q^{n}$.

Proof. By Theorem 4.2.3, each $P_{i}$ is a CPP over $\mathbb{F}_{q^{n}}$ since each $\bar{f}_{i}$ is a CPP over $\mathbb{F}_{q}$ by assumption. Moreover, if $i \neq j$,

$$
\left(P_{j}-P_{i}\right)(x)=x\left[\left(a_{j}-a_{i}\right) x^{p^{r}-1}-\left(a_{j}-a_{i}\right) T_{q^{n} \mid q}(x)^{p^{r}-1}+\left(b_{j}-b_{i}\right)\right]
$$

is a permutation over $\mathbb{F}_{q^{n}}$ by Corollary $4.2 .5\left(\right.$ since $\left.b_{j} \neq b_{i}\right)$.

Corollary 4.2.8 generalizes [63, Theorem 5.5]. 


\subsection{Inverse of the complete mapping}

In this section we obtain the compositional inverse of the complete mapping of Theorem 4.2.3. See Theorem 4.3.2 and Corollary 4.3.3 for this. To achieve this, we make use of the result in Section 4.1 of the compositional inverses of linearized binomials permuting the kernel of the trace map.

Lemma 4.3.1 (Corollary 3.14, [70]). Using the same notations of Lemma 4.2.1 and assuming that $f$ permutes $\mathbb{F}_{q^{n}}$, the following two results hold:

(i) If $p \mid n$ or $x \in \mathbb{F}_{q^{n}}$ is such that $\varphi_{\bar{f}^{-1}(T(x))}$ permutes $\mathbb{F}_{q}$, then $\varphi_{\bar{f}^{-1}(T(x))}$ permutes $\mathbb{F}_{q^{n}}$, and the preimage of $x$ under $f$ is given by

$$
f^{-1}(x)=\varphi_{\bar{f}^{-1}(T(x))}^{-1}(x)
$$

where $\bar{f}^{-1}:=\left.\bar{f}\right|_{\mathbb{F}_{q}} ^{-1}$.

(ii) If $p \nmid n$, then the compositional inverse of $f$ over $\mathbb{F}_{q^{n}}$ is given by

$$
f^{-1}(x)=n^{-1} \bar{f}^{-1}(T(x))+\left.\varphi_{\bar{f}^{-1}(T(x))}\right|_{\operatorname{ker}(T)} ^{-1}\left(x-n^{-1} T(x)\right) .
$$

Recall that $1 / f:=f^{q-2}$ for a polynomial $f$ if $\operatorname{im}(f) \subseteq \mathbb{F}_{q}$ when $f$ is viewed as a mapping.

Theorem 4.3.2. Assume that the polynomial $f$ of Theorem 4.2.3 is a permutation polynomial over $\mathbb{F}_{q^{n}}$. Then $\bar{f}(x):=x G(x)$ is a permutation polynomial over $\mathbb{F}_{q}$ and $\varphi_{y}(x):=a x^{p^{r}}+x\left(G(y)-a y^{p^{r}-1}\right)$ induces a permutation of $\operatorname{ker}(T)$ for each $y \in \mathbb{F}_{q}$. Let $\bar{f}^{-1} \in \mathbb{F}_{q}[x]$ denote the compositional inverse of $\bar{f}$ over $\mathbb{F}_{q}$ and define the polynomial

$$
C(x):=\bar{f}^{-1}(T(x))^{p^{r}-1}-a^{-1} G\left(\bar{f}^{-1}(T(x))\right) \in \mathbb{F}_{q}[x]
$$


Then the following three results hold:

(i) If $x \in \mathbb{F}_{q^{n}}$ is such that $C(x)=0$, then the preimage of $x$ under $f$ is given by

$$
f^{-1}(x)=\left(\frac{x}{a}\right)^{\frac{q^{n}}{p^{r}}}
$$

(ii) Otherwise if $x \in \mathbb{F}_{q^{n}}$ is such that $N_{q \mid p^{d}}(C(x)) \neq 1$, then $N_{q \mid p^{d}}(C(x))^{n} \neq 1$, and the preimage of $x$ under $f$ is given by

$$
f^{-1}(x)=\frac{N_{q \mid p^{d}}(C(x))^{n}}{1-N_{q \mid p^{d}}(C(x))^{n}} \sum_{i=0}^{\frac{m n}{d}-1} C(x)^{-\frac{p^{(i+1) r}-1}{p^{r}-1}}\left(a^{-1} x\right)^{p^{i r}} .
$$

(iii) Otherwise the preimage of $x \in \mathbb{F}_{q^{n}}$ under $f$ is given by

$$
f^{-1}(x)=n^{-1}\left(\bar{f}^{-1}(T(x))+\sum_{j=0}^{\frac{m}{d}-1} C(x)^{-\frac{p^{(j+1) r}-1}{p^{r}-1}}\left(a^{-1} \sum_{k=1}^{n-1} k x^{p^{k m \frac{r}{d}}}\right)^{p^{j r}}\right) .
$$

Proof. Write $f(x)=a x^{p^{r}}-x g(T(x))$ where $g(x):=a x^{p^{r}-1}-G(x)$. As we have seen in the proof of Theorem 4.2.3, the fact that $f$ is a PP over $\mathbb{F}_{q^{n}}$ implies that $\bar{f}(x):=x G(x)$ is a $\mathrm{PP}$ over $\mathbb{F}_{q}$ and $\varphi_{y}(x):=a x^{p^{r}}-x g(y)=a\left(x^{p^{r}}-x g(y) / a\right)=a L_{g(y) / a, r}(x)$ induces a permutation of $\operatorname{ker}(T)$ for each $y \in \mathbb{F}_{q}$. Note that $\left.\varphi_{y}\right|_{\operatorname{ker}(T)} ^{-1}(x)=\left.L_{g(y) / a, r}\right|_{\operatorname{ker}(T)} ^{-1}\left(a^{-1} x\right)$, where $L_{c, r}(x):=x^{p^{r}}-c x$ for $c \in \mathbb{F}_{q}$. Moreover $C(x)=g\left(\bar{f}^{-1}(T(x))\right) / a$.

(i): If $x \in \mathbb{F}_{q^{n}}$ is such that $C(x)=0$, then $\varphi_{\bar{f}^{-1}(T(x))}(x)=a L_{C(x), r}(x)=a x^{p^{r}}$ permutes $\mathbb{F}_{q^{n}}$. Now Lemma 4.3.1 (i) gives

$$
f^{-1}(x)=\varphi_{\bar{f}^{-1}(T(x))}^{-1}(x)=\left.L_{g(y) / a, r}\right|^{-1}\left(a^{-1} x\right)=\left(\frac{x}{a}\right)^{\frac{q^{n}}{p^{r}}} .
$$

(ii) Otherwise if $x \in \mathbb{F}_{q^{n}}$ is such that $N_{q \mid p^{d}}(C(x)) \neq 1$, then, by similar arguments 
to those in the proof of Corollary 4.1.10, we get

$$
N_{q^{n} \mid p^{d}}\left(\frac{g\left(\bar{f}^{-1}(T(x))\right)}{a}\right)=N_{q \mid p^{d}}\left(\frac{g\left(\bar{f}^{-1}(T(x))\right)}{a}\right)^{n} \neq 1 .
$$

Then by Corollary 4.1.2, $L_{g\left(\bar{f}^{-1}(T(x))\right) / a, r}$, and hence $\varphi_{\bar{f}^{-1}(T(x))}$, permute $\mathbb{F}_{q^{n}}$. Since $\varphi_{y}\left(\mathbb{F}_{q}\right) \subseteq \mathbb{F}_{q}$ for each $y \in \mathbb{F}_{q}$, necessarily $\varphi_{\bar{f}^{-1}(T(x))}$ permutes $\mathbb{F}_{q}$. Thus we can apply Lemma 4.3.1 (i) to obtain

$$
f^{-1}(x)=\varphi_{\bar{f}^{-1}(T(x))}^{-1}(x)=L_{g\left(\bar{f}^{-1}(T(x))\right) / a, r}^{-1}\left(a^{-1} x\right) .
$$

Substituting $c$ with $g\left(\bar{f}^{-1}(T(x))\right) / a$ in Corollary 4.1.2, we get

$$
f^{-1}(x)=\frac{N_{q^{n} \mid p^{d}}\left(\frac{g\left(\bar{f}^{-1}(T(x))\right)}{a}\right)}{1-N_{q^{n} \mid p^{d}}\left(\frac{g\left(\bar{f}^{-1}(T(x))\right)}{a}\right)} \sum_{i=0}^{\frac{n m}{d}-1}\left(\frac{g\left(\bar{f}^{-1}(T(x))\right)}{a}\right)^{-\frac{p^{(i+1) r}-1}{p^{r}-1}}\left(a^{-1} x\right)^{p^{i r}} .
$$

Now the result follows from the fact that $N_{q^{n} \mid p^{d}}(y)=N_{q \mid p^{d}}(y)^{n}$ for any $y \in \mathbb{F}_{q}$.

(iii) Here we can use Theorem 4.1.6 to obtain the inverse of $\left.L_{g\left(\bar{f}^{-1}(T(x))\right) / a, r}\right|_{\operatorname{ker}(T)}$. Since $L_{g\left(\bar{f}^{-1}(T(x))\right) / a, r}$, and hence $\varphi_{\bar{f}^{-1}(T(x))}$, do not permute $\mathbb{F}_{q^{n}}$, we apply Lemma 4.3.1 (ii) instead. This gives

$$
\begin{aligned}
f^{-1}(x) & =n^{-1} \bar{f}^{-1}(T(x))+\left.\varphi_{\bar{f}^{-1}(T(x))}\right|_{\operatorname{ker}(T)} ^{-1}\left(x-n^{-1} T(x)\right) \\
& =n^{-1} \bar{f}^{-1}(T(x))+\left.L_{g\left(\bar{f}^{-1}(T(x))\right) / a, r}\right|_{\operatorname{ker}(T)} ^{-1}\left(a^{-1}\left(x-n^{-1} T(x)\right)\right)
\end{aligned}
$$

For the sake of brevity denote $\bar{L}(z):=\left.L_{g\left(\bar{f}^{-1}(T(x))\right) / a, r}\right|_{\operatorname{ker}(T)} ^{-1}(z) \in \mathbb{F}_{q}[z]$. Since $\bar{L}$ is a p-polynomial, the above becomes

$$
f^{-1}(x)=n^{-1} \bar{f}^{-1}(T(x))+\bar{L}\left(a^{-1} x\right)-\bar{L}\left(a^{-1} n^{-1} T(x)\right) .
$$


By the definition of $\bar{L}$ and by Theorem 4.1.6 with $c=g\left(\bar{f}^{-1}(T(x))\right) / a$, we get

$$
\begin{aligned}
\bar{L}\left(a^{-1} n^{-1} T(x)\right) & =\sum_{j=0}^{\frac{m}{d}-1}\left(\frac{g\left(\bar{f}^{-1}(T(x))\right)}{a}\right)^{-\frac{p^{(j+1) r}-1}{p^{r}-1}}\left(n^{-2} \sum_{k=1}^{n-1} k a^{-1} T(x)\right)^{p^{j r}} \\
& =\frac{n^{-1}(n-1)}{2} \sum_{j=0}^{\frac{m}{d}-1}\left(\frac{g\left(\bar{f}^{-1}(T(x))\right)}{a}\right)^{-\frac{p^{(j+1) r}-1}{p^{r}-1}}\left(a^{-1} T(x)\right)^{p^{j r}} .
\end{aligned}
$$

Since $\bar{f}(y)=y G(y)=a y^{p^{r}}-y g(y)$ for any $y \in \mathbb{F}_{q}$, we have $y=a \bar{f}^{-1}(y)^{p^{r}}-$ $\bar{f}^{-1}(y) g\left(\bar{f}^{-1}(y)\right)$. Then, if we substitute $y$ with $T(x)$, the above becomes

$$
\begin{aligned}
\bar{L}\left(a^{-1} n^{-1} T(x)\right)= & \frac{n^{-1}(n-1)}{2} \sum_{j=0}^{\frac{m}{d}-1}\left(\frac{g\left(\bar{f}^{-1}(T(x))\right)}{a}\right)^{-\frac{p^{(j+1) r}-1}{p^{r}-1}} \\
\cdot\left(\bar{f}^{-1}(T(x))^{p^{r}}-\bar{f}^{-1}(T(x)) \frac{g\left(\bar{f}^{-1}(T(x))\right)}{a}\right)^{p^{j r}} & \left.-\bar{f}^{-1}(T(x))^{p^{j r}}\left(\frac{g\left(\bar{f}^{-1}(T(x))\right)}{a}\right)^{-\frac{p^{j r}-1}{p^{r}-1}}\right) \\
& =\frac{n^{-1}(n-1)}{2} \sum_{j=0}^{\frac{m}{d}-1}\left(\bar{f}^{-1}(T(x))^{p^{(j+1) r}}\left(\frac{g\left(\bar{f}^{-1}(T(x))\right)}{a}\right)^{-\frac{p^{(j+1) r}-1}{p^{r}-1}}\right. \\
& =\frac{n^{-1}(n-1)}{2} \bar{f}^{-1}(T(x))\left(\left(\frac{g\left(\bar{f}^{-1}(T(x))\right)}{a}\right)^{-\frac{p^{m r / d}-1}{p^{r}-1}}-1\right) \\
& =0
\end{aligned}
$$

because, by Lemma 4.1.3 (ii) and by assumption,

$$
\left(\frac{g\left(\bar{f}^{-1}(T(x))\right)}{a}\right)^{\frac{p^{m r / d}-1}{p^{r}-1}}=\left(\frac{g\left(\bar{f}^{-1}(T(x))\right)}{a}\right)^{\frac{p^{m}-1}{p^{d}-1}}=1 .
$$


Thus

$$
\begin{aligned}
f^{-1}(x) & =n^{-1} \bar{f}^{-1}(T(x))+\left.L_{g\left(\bar{f}^{-1}(T(x))\right) / a, r}\right|_{\operatorname{ker}(T)} ^{-1}\left(a^{-1} x\right) \\
& =n^{-1} \bar{f}^{-1}(T(x))+\sum_{j=0}^{\frac{m}{d}-1}\left(\frac{g\left(\bar{f}^{-1}(T(x))\right)}{a}\right)^{-\frac{p^{(j+1) r}-1}{p^{\gamma}-1}}\left(n^{-1} \sum_{k=1}^{n-1} k a^{-1} x^{p^{k m \frac{r}{d}}}\right)^{p^{j r}} .
\end{aligned}
$$

The result follows.

Corollary 4.3.3. If the polynomial $f$ in Theorem 4.2.3 is a permutation polynomial over $\mathbb{F}_{q^{n}}$, then its compositional inverse over $\mathbb{F}_{q^{n}}$ is given by

$$
\begin{aligned}
f^{-1}(x)= & \left(1-C(x)^{q-1}\right)\left(\frac{x}{a}\right)^{\frac{q^{n}}{p^{r}}} \\
& +C(x)^{q-1}\left(N_{q \mid p^{d}}(C(x))-1\right)^{p^{d}-1} \frac{N_{q \mid p^{d}}(C(x))^{n}}{1-N_{q \mid p^{d}}(C(x))^{n}} \\
& \cdot \sum_{i=0}^{\frac{m n}{d}-1} C(x)^{\frac{-p^{(i+1) r}-1}{p^{r}-1}}\left(a^{-1} x\right)^{p^{i r}} \\
& +\left(1-\left(N_{q \mid p^{d}}(C(x))-1\right)^{p^{d}-1}\right) \\
& \cdot n^{-1}\left(\bar{f}^{-1}(T(x))+\sum_{j=0}^{\frac{m}{d}-1} C(x)^{-\frac{p^{(j+1) r}-1}{p^{r}-1}}\left(a^{-1} \sum_{k=1}^{n-1} k x^{p^{k m m}}\right)^{p^{j r}}\right) .
\end{aligned}
$$

Proof. We put the results of Theorem 4.3.2 together. Note that this is a step function where only one of the three terms is non-zero at a time. The first term of the expression is non-zero only when $C(x)=0$ corresponding to the result in (i), while the second term is non-zero only when $C(x) \neq 0$ and $N_{q \mid p^{d}}(C(x)) \neq 1$ corresponding to (ii). The third term is non-zero otherwise; this corresponds to the result in (iii). These are the only possibilities for $C(x)$ and so we are done.

Corollary 4.3.3 generalizes [70, Corollary 4.6] and [85, Theorem 3.5]. 


\section{Chapter 5}

\section{A new proof of the Hansen-Mullen irreducibility conjecture}

In this chapter ${ }^{1}$ we present a new proof of the Hansen-Mullen irreducibility conjecture, or theorem, stated in Theorem 1.0.11.

The content of this chapter is as follows. In Section 5.1 we study the connection, between the least period of the DFT of functions, and irreducible polynomials. In particular we explicitly describe in Proposition 5.1.1 the least period of the DFT of functions, as well as prove Lemmas 1.0.12 and 1.0.13. In Section 5.2 we introduce the characteristic delta functions as the DFTs of characteristic elementary symmetric functions. We then apply Lemma 1.0.13 to give a sufficient condition, in Lemma 5.2 .2 , for the existence of an irreducible polynomial with any one of its coefficients prescribed. This is given in terms of the least period of a certain function $\Delta_{w, c}$, closely

\footnotetext{
${ }^{1}$ This chapter is substantively based on the following article first published in Canadian Journal of Mathematics at https://doi.org/10.4153/CJM-2017-022-1, Copyright (C) 2018, Canadian Mathematical Society. It is released under a Creative Commons Attribution Non-Commercial No Derivatives License.

[72] A. Tuxanidy and Q. Wang, A new proof of the Hansen-Mullen irreducibility conjecture, Canadian Journal of Mathematics, no.70 (2018), 1373-1389. https://doi.org/10.4153/ CJM-2017-022-1
} 
related to the delta functions. We also review some basic results on $q$-symmetric functions and their convolutions; this will be needed in Section 5.3. Finally in Section 5.3 we prove that the $\Delta_{w, c}$ functions have period that are not divisors of $\left(q^{n}-1\right) / \Phi_{n}(q)$ (and are sufficiently large in many cases). The proof of Theorem 1.0.11 then immediately follows from this.

\subsection{Least period of the DFT and connection to irreducible polynomials}

In this section we study a connection between the least period of the DFT of a function and irreducible polynomials. We start off by giving an explicit formula in Proposition 5.1.1 for the least period of the DFT of a function $f: \mathbb{Z}_{N} \rightarrow \mathbb{F}_{q}$ in terms of the values in its support. Then we prove Lemmas 1.0.12 and 1.0.13.

First we may identify, in the usual way, elements of $\mathbb{Z}_{N}=\mathbb{Z} / N \mathbb{Z}$ with their canonical representatives in $\mathbb{Z}$ and vice versa. In particular this endows $\mathbb{Z}_{N}$ with the natural ordering in $\mathbb{Z}$. We may also sometimes abuse notation and write $a \mid \bar{b}$ for $a \in \mathbb{Z}$ and $\bar{b} \in \mathbb{Z}_{N}$ to state that $a$ divides the canonical representative of $\bar{b}$, and write $a \nmid \bar{b}$ to state the opposite. For an integer $k$ and a non-empty set $A=\left\{a_{1}, \ldots, a_{s}\right\}$, we write $\operatorname{gcd}(k, A):=\operatorname{gcd}\left(k, a_{1}, \ldots, a_{s}\right)$.

Proposition 5.1.1. Let $q$ be a power of a prime, let $N \mid q-1$, let $f: \mathbb{Z}_{N} \rightarrow \mathbb{F}_{q}$ and let $\zeta_{N}$ be a primitive $N$-th root of unity in $\mathbb{F}_{q}^{*}$. The least period of $\mathcal{F}_{\zeta_{N}}[f]$ and $\mathcal{F}_{\zeta_{N}}^{-1}[f]$ is given by $N / \operatorname{gcd}(N, \operatorname{supp}(f))$.

Proof. Note that $d:=N / \operatorname{gcd}(N, \operatorname{supp}(f))$ is the smallest positive divisor of $N$ with the property that $N / d$ divides every element in $\operatorname{supp}(f)$. For the sake of brevity write 
$\widehat{f}=\mathcal{F}_{\zeta_{N}}[f]$. Now for $i \in \mathbb{Z}_{N}$ note that

$$
\begin{aligned}
\widehat{f}(i+d) & =\sum_{j \in \mathbb{Z}_{N}} f(j) \zeta_{N}^{(i+d) j}=\sum_{k=0}^{d-1} f\left(\frac{N}{d} k\right) \zeta_{N}^{(i+d) \frac{N}{d} k}=\sum_{k=0}^{d-1} f\left(\frac{N}{d} k\right) \zeta_{N}^{i \frac{N}{d} k} \\
& =\widehat{f}(i) .
\end{aligned}
$$

Thus if $r$ is the least period of $\widehat{f}$, necessarily $r \leq d$.

Since $f=\mathcal{F}_{\zeta_{N}}^{-1}[\widehat{f}]$, then

$$
f(i)=N^{-1} \sum_{j \in \mathbb{Z}_{N}} \widehat{f}(j) \zeta_{N}^{-i j}, \quad i \in \mathbb{Z}_{N}
$$

Hence for $i \in \mathbb{Z}_{N}$ we have

$$
\begin{aligned}
N f(i) & =\sum_{j \in \mathbb{Z}_{N}} \widehat{f}(j) \zeta_{N}^{-i j}=\sum_{j=0}^{r-1} \widehat{f}(j) \zeta_{N}^{-i j}+\sum_{j=r}^{2 r-1} \widehat{f}(j) \zeta_{N}^{-i j}+\cdots+\sum_{j=\left(\frac{N}{r}-1\right) r}^{N-1} \widehat{f}(j) \zeta_{N}^{-i j} \\
& =\sum_{j=0}^{r-1} \widehat{f}(j) \zeta_{N}^{-i j}+\sum_{j=0}^{r-1} \widehat{f}(j+r) \zeta_{N}^{-i(j+r)}+\cdots+\sum_{j=0}^{r-1} \widehat{f}\left(j+\left(\frac{N}{r}-1\right) r\right) \zeta_{N}^{-i\left(j+\left(\frac{N}{r}-1\right) r\right)} \\
& =\sum_{j=0}^{r-1} \widehat{f}(j) \zeta_{N}^{-i j}+\zeta_{N}^{-i r} \sum_{j=0}^{r-1} \widehat{f}(j) \zeta_{N}^{-i j}+\cdots+\zeta_{N}^{-i\left(\frac{N}{r}-1\right) r} \sum_{j=0}^{r-1} \widehat{f}(j) \zeta_{N}^{-i j} \\
& =\sum_{k=0}^{\frac{N}{r}-1} \zeta_{N}^{-i r k} \sum_{j=0}^{r-1} \widehat{f}(j) \zeta_{N}^{-i j}
\end{aligned}
$$

If $\frac{N}{r} \nmid i$, then $\zeta_{N}^{-i r} \neq 1$ and $\sum_{k=0}^{\frac{N}{r}-1} \zeta_{N}^{-i r k}=\frac{\zeta_{N}^{-i N}-1}{\zeta_{N}^{-i r}-1}=0$. It follows that $f(i)=0$ whenever $\frac{N}{r} \nmid i$. Equivalently, if $f(i) \neq 0$, then $\frac{N}{r} \mid i$. Now the minimality of $d$ implies that $d \leq r$. But $r \leq d$ (see above) now yields $r=d$.

With regards to the least period of $\mathcal{F}_{\zeta_{N}}^{-1}[f]$, we know that $\mathcal{F}_{\zeta_{N}}^{-1}[f]=N^{-1} \mathcal{F}_{\zeta_{N}^{-1}}[f]$. Since $\zeta_{N}^{-1}$ is a primitive $N$-th root of unity in $\mathbb{F}_{q}^{*}$ as well, the previous arguments simi- 
larly imply that $\mathcal{F}_{\zeta_{N}^{-1}}[f]$ has the least period $d$. Then so does the function $N^{-1} \mathcal{F}_{\zeta_{N}^{-1}}[f]$, a non-zero scalar multiple of $\mathcal{F}_{\zeta_{N}^{-1}}[f]$.

In particular, if $\zeta$ is primitive in $\mathbb{F}_{q}$ and $F(x)=\sum_{i \in I} a_{i} x^{i} \in \mathbb{F}_{q}[x]$ for some subset $I \subseteq[0, q-2]$ of integers with each $a_{i} \neq 0, i \in I$, then the least period of the $(q-1)$ periodic sequence $\left(F\left(\zeta^{i}\right)\right)_{i \geq 0}$ is given by $(q-1) / \operatorname{gcd}(q-1, I)$. We now prove Lemma 1.0.12.

Proof of Lemma 1.0.12. (i) On the contrary, suppose that $\operatorname{supp}(F)$ contains no element of degree $n$ over $\mathbb{F}_{q}$. Then for each $m \in \operatorname{supp}(f)$ there exists a proper divisor $d$ of $n$ with $\left(q^{n}-1\right) /\left(q^{d}-1\right) \mid m$. Since $\Phi_{n}(q) \mid\left(q^{n}-1\right) /\left(q^{d}-1\right)$ for all proper divisors $d$ of $n$, then $\Phi_{n}(q) \mid m$ for all $m \in \operatorname{supp}(f)$. Thus for all $k \in \mathbb{Z}_{q^{n}-1}$,

$$
\hat{f}(k)=\sum_{j \in \mathbb{Z}_{q^{n}-1}} f(j) \zeta^{k j}=\sum_{a=1}^{\left(q^{n}-1\right) / \Phi_{n}(q)} f\left(a \Phi_{n}(q)\right) \zeta^{k a \Phi_{n}(q)},
$$

where $\hat{f}=\mathcal{F}_{\zeta}[f]$. Note that $\hat{f}\left(k+\left(q^{n}-1\right) / \Phi_{n}(q)\right)=\hat{f}(k)$ for all $k \in \mathbb{Z}_{q^{n}-1}$. Thus $\hat{f}$ is $\frac{q^{n}-1}{\Phi_{n}(q)}$-periodic. Necessarily the least period of $\hat{f}$ divides $\frac{q^{n}-1}{\Phi_{n}(q)}$, a contradiction.

(ii) Assume $\operatorname{supp}(F)$ contains an element of degree $n$ over $\mathbb{F}_{q}$. Then there exists $m \in \operatorname{supp}(f)$ with $\left(q^{n}-1\right) /\left(q^{d}-1\right) \nmid m$ for all proper divisors $d$ of $n$. Let $r$ be the least period of $\hat{f}$. By Proposition 5.1.1, $\left(q^{n}-1\right) / r \mid m$. Since $\left(q^{n}-1\right) /\left(q^{d}-1\right) \nmid m$, then $r \nmid q^{d}-1$ for all proper divisors $d$ of $n$.

(iii) Assume $\operatorname{supp}(F)$ contains a primitive element of $\mathbb{F}_{q^{n}}$. Then there exists $k$ relatively prime to $q^{n}-1$ such that $\bar{k} \in \operatorname{supp}(f)$. Thus $\left(\mathbb{Z} /\left(q^{n}-1\right) \mathbb{Z}\right)^{\times} \cap \operatorname{supp}(f) \neq \emptyset$. It follows from Proposition 5.1.1 that both $\mathcal{F}_{\zeta}[f]$ and $\mathcal{F}_{\zeta}^{-1}[f]$ have maximum least period $q^{n}-1$. The second part follows similarly. 
Note that, as the following three examples show, the sufficient (respectively necessary) conditions in Lemma 1.0.12 are not necessary (respectively sufficient). These may possibly be improved in accordance with the needs of whoever wishes to apply these tools. Let us start off by showing that the sufficient condition in (i) is not necessary.

Example 5.1.2. Recall that $\left(q^{n}-1\right) / \Phi_{n}(q)=\operatorname{lcm}\left\{q^{d}-1: d \mid n, d<n\right\}$. Pick any $n$ with at least two prime factors. Then $\left(q^{n}-1\right) / \Phi_{n}(q) \nmid q^{d}-1$ for all $d \mid n$, $d<n$. Thus $\zeta^{\Phi_{n}(q)}$ is of degree $n$ over $\mathbb{F}_{q}$. Define the function $F: \mathbb{F}_{q^{n}} \rightarrow \mathbb{F}_{q^{n}}$ by $F\left(\zeta^{\Phi_{n}(q)}\right)=1$ and $F(\xi)=0$ for all other elements $\xi \in \mathbb{F}_{q^{n}}$. Thus $\operatorname{supp}(F)$ contains an element of degree $n$ over $\mathbb{F}_{q}$. The associate function $f: \mathbb{Z}_{q^{n}-1} \rightarrow \mathbb{F}_{q^{n}}$ is defined by $f(k)=1$ if $k=\Phi_{n}(q)$ and $f(k)=0$ otherwise. By Proposition 5.1.1, the least period $r$ of $\mathcal{F}_{\zeta}[f]$ is the smallest positive divisor of $q^{n}-1$ such that $\left(q^{n}-1\right) / r \mid \Phi_{n}(q)$, since $\operatorname{supp}(f)=\left\{\Phi_{n}(q)\right\}$. This is $r=\left(q^{n}-1\right) / \Phi_{n}(q)$. Thus we obtain an example of a function which contains an element of degree $n$ over $\mathbb{F}_{q}$ in its support but for which the corresponding least period is a divisor of $\left(q^{n}-1\right) / \Phi_{n}(q)$.

The following example shows that the necessary condition in (ii) is not sufficient.

Example 5.1.3. Similarly as before, pick any $n$ with at least two prime factors. Then $\left(q^{n}-1\right) / \Phi_{n}(q) \nmid q^{d}-1$ for all $d \mid n, d<n$. Define $F: \mathbb{F}_{q^{n}} \rightarrow \mathbb{F}_{q^{n}}$ by $F\left(\zeta^{k}\right)=1$ if $k=\left(q^{n}-1\right) /\left(q^{d}-1\right)$ for some $d \mid n, d<n$, and $F(\xi)=0$ for all other elements $\xi \in \mathbb{F}_{q^{n}}$. Thus $\operatorname{supp}(F)$ has no element of degree $n$ over $\mathbb{F}_{q}$. This defines the associate function $f: \mathbb{Z}_{q^{n}-1} \rightarrow \mathbb{F}_{q^{n}}$ of $F$ with $\operatorname{supp}(f)=\left\{\left(q^{n}-1\right) /\left(q^{d}-1\right): d \mid n, d<n\right\}$. Consider the smallest positive divisor $r$ of $q^{n}-1$, with $\left(q^{n}-1\right) / r \mid\left(q^{n}-1\right) /\left(q^{d}-1\right)$ for all proper divisors $d$ of $n$. Note that $r$ is divisible by each $q^{d}-1$, for $d \mid n, d<n$; it follows $r=\operatorname{lcm}\left\{q^{d}-1: d \mid n, d<n\right\}=\left(q^{n}-1\right) / \Phi_{n}(q)$ with $r \nmid q^{d}-1$ for all $d \mid n$, $d<n$. By Proposition 5.1.1, $r=\left(q^{n}-1\right) / \Phi_{n}(q)$ is the least period of $\mathcal{F}_{\zeta}[f]$. Thus we 
have constructed a function $F$ with $\operatorname{supp}(F)$ having no element of degree $n$ over $\mathbb{F}_{q}$ but for which the corresponding least period $r$ satisfies $r \nmid\left(q^{d}-1\right)$ for all $d \mid n, d<n$.

This last example shows that the necessary condition in (iii) is not sufficient.

Example 5.1.4. Pick $q, n$ such that $q^{n}-1$ has at least two non-trivial relatively prime divisors, say $a, b>1$ with $a, b \mid\left(q^{n}-1\right)$ and $\operatorname{gcd}(a, b)=1$. The smallest positive divisor $r$ of $q^{n}-1$ with $\left(q^{n}-1\right) / r \mid a, b$ is $r=q^{n}-1$. Now we note that the function $F: \mathbb{F}_{q^{n}} \rightarrow \mathbb{F}_{q^{n}}$ defined by $F\left(\zeta^{a}\right)=F\left(\zeta^{b}\right)=1$ and $F(\xi)=0$ for all other elements $\xi$ of $\mathbb{F}_{q^{n}}$, contains no primitive element in its support, but the corresponding least period of $\mathcal{F}_{\zeta}[f]$ is $q^{n}-1$, by Proposition 5.1.1.

Remark 5.1.5. We remark that Example 5.1.4 together with Lemma 1.0.12 (i) imply that for any such $a, b$, there exists $k \in\{a, b\}$ such that $\left(q^{n}-1\right) /\left(q^{d}-1\right) \nmid k$ for all proper divisors $d$ of $n$; that is, either $\zeta^{a}$ or $\zeta^{b}$ (or both) is an element of degree $n$ over $\mathbb{F}_{q}$. This may also have applications in determining whether a polynomial $h(x) \in \mathbb{F}_{q}[x]$ has an irreducible factor of degree $n$. Specifically, if there exist divisors $a, b \geq 1$ of $q^{n}-1$ with $\operatorname{gcd}(a, b)=1$ and $h\left(\zeta^{a}\right)=h\left(\zeta^{b}\right)=0$, then $h(x)$ has an irreducible factor of degree $n$.

Finally we prove Lemma 1.0.13.

Proof of Lemma 1.0.13. As a function on $\mathbb{F}_{q^{n}}^{\times}$, note that

$$
S(\xi)= \begin{cases}1 & \text { if } h(\xi)=0 \\ 0 & \text { otherwise }\end{cases}
$$

Let $\zeta$ be a primitive element of $\mathbb{F}_{q^{n}}$ and define the function $f: \mathbb{Z}_{q^{n}-1} \rightarrow \mathbb{F}_{q}$ by $f(m)=s_{m}$. Thus $f$ has least period $r$ satisfying $r \nmid\left(q^{n}-1\right) / \Phi_{n}(q)$. Note that 
$S\left(\zeta^{i}\right)=\sum_{j} s_{j} \zeta^{i j}=\sum_{j} f(j) \zeta^{i j}=\mathcal{F}_{\zeta}[f](i)$ for each $i \in \mathbb{Z}_{q^{n}-1}$. Since $\mathcal{F}_{\zeta}[S]$ has the same period as $\mathcal{F}_{\zeta}^{-1}[S]$ (the inverse is essentially a Fourier multiplied by a non-zero scalar), then by the criteria (i) of Lemma 1.0.12, there exists an element of degree $n$ over $\mathbb{F}_{q}$ in the support of $S$. It follows $h(x)$ has a root of degree $n$ over $\mathbb{F}_{q}$ and hence has an irreducible factor of degree $n$ over $\mathbb{F}_{q}$.

\subsection{Characteristic elementary symmetric and delta functions}

In this section we apply Lemma 1.0.13 for the purposes of studying coefficients of irreducible polynomials. We first place the characteristic elementary symmetric functions in the context of their DFT, which we shall refer to here simply as delta functions. These delta functions are indicators, with values in a finite field, for sets of values in $\mathbb{Z}_{q^{n}-1}$ whose canonical integer representatives have certain Hamming weights in their $q$-adic representation and $q$-digits all belonging to the set $\{0,1\}$. Essentially, characteristic elementary symmetric functions are characteristic generating functions of the sets that the delta functions indicate. Then we give in Lemma 5.2.2 sufficient conditions for an irreducible polynomial to have a prescribed coefficient. Because the delta functions are $q$-symmetric (see Definition 5.2.3), we also review some useful facts needed in Section 5.3.

For $\xi \in \mathbb{F}_{q^{n}}$, the characteristic polynomial $h_{\xi}(x) \in \mathbb{F}_{q}[x]$ of degree $n$ over $\mathbb{F}_{q}$ with root $\xi$ is given by

$$
h_{\xi}(x)=\prod_{k=0}^{n-1}\left(x-\xi^{q^{k}}\right)=\sum_{w=0}^{n}(-1)^{w} \sigma_{w}(\xi) x^{n-w}
$$

where for $0 \leq w \leq n, \sigma_{w}(x) \in \mathbb{F}_{q}[x]$ is the characteristic elementary symmetric 
polynomial given by $\sigma_{0}(x)=1$ and

$$
\sigma_{w}(x)=\sum_{0 \leq i_{1}<\cdots<i_{w} \leq n-1} x^{q^{i_{1}+\cdots+q^{i w}}}
$$

for $1 \leq w \leq n$. In particular $\sigma_{1}=\operatorname{Tr}_{\mathbb{F}_{q^{n} / \mathbb{F}_{q}}}$ is the (linear) trace function and $\sigma_{n}=N_{\mathbb{F}_{q^{n}} / \mathbb{F}_{q}}$ is the (multiplicative) norm function. Whenever $q=2$ and $\xi \neq 0$, then $\sigma_{0}(\xi)=\sigma_{n}(\xi)=1$ always. If $\xi \neq 0$, then (in general) $h_{\xi^{-1}}(x)=(-1)^{n} \sigma_{n}\left(\xi^{-1}\right) x^{n} h_{\xi}(1 / x)=$ $h_{\xi}^{*}(x)$, where $h_{\xi}^{*}(x)$ is the (monic) reciprocal of $h_{\xi}(x)$. Thus $\sigma_{w}(\xi)=\sigma_{n}(\xi) \sigma_{n-w}\left(\xi^{-1}\right)$. Clearly $h_{\xi}(x)$ is irreducible if and only if so is $h_{\xi}^{*}(x)$. This occurs if and only if $\operatorname{deg}_{\mathbb{F}_{q}}(\xi)=n$.

Next we introduce the characteristic delta functions and the sets they indicate. But first let us clarify some ambiguity in our notation: For $a, b \in \mathbb{Z}$, we denote by $a \bmod b$ the remainder of division of $a$ by $b$. That is, $a \bmod b$ is the smallest integer $c$ in $\{0,1, \ldots, b-1\}$ that is congruent to $a$ modulo $b$, and write $c=a \bmod b$. Similarly if $\bar{a}=a+b \mathbb{Z}$ is an element of $\mathbb{Z}_{b}$, we use the notation $\bar{a} \bmod b:=a \bmod b$ to express the canonical representative of $\bar{a}$ in $\mathbb{Z}$. But we keep the usual notation $k \equiv a(\bmod b)$ to state that $b \mid(k-a)$.

We can represent $a \in \mathbb{Z}_{q^{n}-1}$ uniquely by the $q$-adic representation $\left(a_{0}, \ldots, a_{n-1}\right)_{q}=$ $\sum_{i=0}^{n-1} a_{i} q^{i}$, with each $0 \leq a_{i} \leq q-1$, of the canonical representative of $a$ in $\left\{0,1, \ldots, q^{n}-\right.$ $2\} \subset \mathbb{Z}$. For the sake of convenience we write $a=\left(a_{0}, \ldots, a_{n-1}\right)_{q}$. For $w \in[0, n]:=$ $\{0,1, \ldots, n\}$, define the sets $\Omega(w) \subseteq \mathbb{Z}_{q^{n}-1}$ by $\Omega(0)=\{0\}$ and

$$
\Omega(w)=\left\{k \in \mathbb{Z}_{q^{n}-1}: k \bmod \left(q^{n}-1\right)=q^{i_{1}}+\cdots+q^{i_{w}}, 0 \leq i_{1}<\cdots<i_{w} \leq n-1\right\}
$$

for $1 \leq w \leq n$. That is, $\Omega(w)$ consists of all the elements $k \in \mathbb{Z}_{q^{n}-1}$ whose canonical representatives in $\left\{0,1, \ldots, q^{n}-2\right\} \subset \mathbb{Z}$ have Hamming weight $w$ in their $q$-adic rep- 
resentation $\left(a_{0}, \ldots, a_{n-1}\right)_{q}=\sum_{i=0}^{n-1} a_{i} q^{i}$, with each $a_{i} \in\{0,1\}$. Note this last condition that each $a_{i} \in\{0,1\}$ is automatically redundant when $q=2$, since in general each $a_{i} \in[0, q-1]$ in the $q$-adic representation $t=\left(a_{0}, \ldots, a_{m}\right)_{q}$ of a non-negative integer $t=\sum_{i=0}^{m} a_{i} q^{i}$

When $q=2$, note $\Omega(n)=\emptyset$ since there is no integer in $\left\{0,1, \ldots, 2^{n}-2\right\}$ with Hamming weight $n$ in its binary representation. Observe also that $|\Omega(w)|=\left(\begin{array}{l}n \\ w\end{array}\right)$ for each $0 \leq w \leq n$, unless $(q, w)=(2, n)$. Moreover $\Omega(v) \cap \Omega(w)=\emptyset$ whenever $v \neq w$, by the uniqueness of base representation of integers. For $w \in[0, n]$, define the characteristic (finite field valued) function $\delta_{w}: \mathbb{Z}_{q^{n}-1} \rightarrow \mathbb{F}_{p}$ of the set $\Omega(w)$ by

$$
\delta_{w}(k)= \begin{cases}1 & \text { if } k \in \Omega(w) \\ 0 & \text { otherwise }\end{cases}
$$

Observe that our $\delta_{0}$ is the Kronecker delta function on $\mathbb{Z}_{q^{n-1}}$ with values in $\{0,1\} \subseteq$ $\mathbb{F}_{p}$

Lemma 5.2.1. Let $\zeta$ be a primitive element of $\mathbb{F}_{q^{n}}$ and let $w \in[0, n]$. If $q=2$, further assume that $w \neq n$. Then

$$
\sigma_{w}\left(\zeta^{k}\right)=\mathcal{F}_{\zeta}\left[\delta_{w}\right](k), \quad k \in \mathbb{Z}_{q^{n}-1}
$$

Proof. Note $\sigma_{0}\left(\zeta^{k}\right)=1$ for each $k$ and so $\sigma_{0}\left(\zeta^{k}\right)=\mathcal{F}_{\zeta}\left[\delta_{0}\right](k)$. Now let $1 \leq w \leq n$. By definition and the assumption that $(q, w) \neq(2, n)$, we have

$$
\begin{aligned}
\sigma_{w}\left(\zeta^{k}\right) & =\sum_{0 \leq i_{1}<\cdots<i_{w} \leq n-1} \zeta^{k\left(q^{i_{1}+\cdots+q^{i w}}\right)}=\sum_{j \in \mathbb{Z}_{q^{n}-1}} \delta_{w}(j) \zeta^{k j} \\
& =\mathcal{F}_{\zeta}\left[\delta_{w}\right](k) .
\end{aligned}
$$


These functions are related to various mathematical objects in literature: Let $m<$ $q$, let $r_{1}, \ldots, r_{m} \in[1, n-1]$, and let $c_{0}, \ldots, c_{n-1} \in[0, m-1]$ such that $\sum_{i=1}^{m} r_{i}=\sum_{j=0}^{n-1} c_{j}$. View each $\delta_{r_{1}}, \ldots, \delta_{r_{m}}$ as having values in $\mathbb{Z}$. Then one can show that

$$
\delta_{r_{1}} \otimes \cdots \otimes \delta_{r_{m}}\left(\left(c_{0}, \ldots, c_{n-1}\right)_{q}\right)
$$

is the number of $m \times n$ matrices, with entries in $\{0,1\} \subset \mathbb{Z}$, such that the sum of the entries in row $i, 1 \leq i \leq m$, is $r_{i}$, and the sum of the entries in column $j, 0 \leq j \leq n-1$, is $c_{j}$. Matrices with $0-1$ entries and prescribed row and column sums are classical objects appearing in numerous branches of pure and applied mathematics, such as combinatorics, algebra and statistics. See for instance the survey in [7] and Chapter 16 in [49].

An application of Lemma 1.0.13 yields the following sufficient condition for the existence of irreducible polynomials with a prescribed coefficient.

Lemma 5.2.2. Fix a prime power $q$ and integers $n \geq 2$ and $1 \leq w \leq n$. Fix $c \in \mathbb{F}_{q}$. If $q=2$, further assume that $w \neq n$. If the function $\Delta_{w, c}: \mathbb{Z}_{q^{n}-1} \rightarrow \mathbb{F}_{q}$ given by

$$
\Delta_{w, c}=\delta_{0}-\left((-1)^{w} \delta_{w}-c \delta_{0}\right)^{\otimes(q-1)}
$$

has least period $r$ satisfying $r \nmid\left(q^{n}-1\right) / \Phi_{n}(q)$, then there exists an irreducible polynomial $P(x)$ of degree $n$ over $\mathbb{F}_{q}$ with $\left[x^{n-w}\right] P(x)=c$.

Proof. Take $h(x)=(-1)^{w} \sigma_{w}(x)-c \in \mathbb{F}_{q}[x]$ in Lemma 1.0.13. Since $\sigma_{w}\left(\mathbb{F}_{q^{n}}\right) \subseteq \mathbb{F}_{q}$, we can pick $L=\mathbb{F}_{q}$. Thus $S(x) \in \mathbb{F}_{q}[x]$ is given by

$$
S(x)=\left[1-\left((-1)^{w} \sigma_{w}(x)-c\right)^{q-1}\right] \bmod \left(x^{q^{n}-1}-1\right) .
$$


Let $\zeta$ be a primitive element of $\mathbb{F}_{q^{n}}$. By Lemma 5.2.1, the linearity of the DFT, and the fact that $c=\mathcal{F}_{\zeta}\left[c \delta_{0}\right]$, we have

$$
S\left(\zeta^{i}\right)=1-\left((-1)^{w} \sigma_{w}\left(\zeta^{i}\right)-c\right)^{q-1}=\mathcal{F}_{\zeta}\left[\delta_{0}\right](i)-\left(\mathcal{F}_{\zeta}\left[(-1)^{w} \delta_{w}-c \delta_{0}\right](i)\right)^{q-1}
$$

Since the product of DFTs is the DFT of the convolution, then, as a function on $\mathbb{F}_{q^{n}}$,

$$
\begin{aligned}
S & =\mathcal{F}_{\zeta}\left[\delta_{0}\right]-\mathcal{F}_{\zeta}\left[\left((-1)^{w} \delta_{w}-c \delta_{0}\right)^{\otimes(q-1)}\right]=\mathcal{F}_{\zeta}\left[\delta_{0}-\left((-1)^{w} \delta_{w}-c \delta_{0}\right)^{\otimes(q-1)}\right] \\
& =\mathcal{F}_{\zeta}\left[\Delta_{w, c}\right] .
\end{aligned}
$$

Thus

$$
S\left(\zeta^{m}\right)=\sum_{i=0}^{q^{n}-2} \Delta_{w, c}(i) \zeta^{m i}
$$

for each $m \in \mathbb{Z}_{q^{n}-1}$. As $S(x)$ is already reduced modulo $x^{q^{n}-1}-1$, it follows (from the uniqueness of the DFT of a function) that $S(x)=\sum_{i=0}^{q^{n}-2} \Delta_{w, c}(i) x^{i}$. Since the least period of $\Delta_{w, c}$ is not a divisor of $\left(q^{n}-1\right) / \Phi_{n}(q)$ by assumption, Lemma 1.0.13 implies $h(x)$ has an irreducible factor $P(x)$ of degree $n$ over $\mathbb{F}_{q}$. Any of the roots $\xi$ of $P(x)$ must satisfy $h(\xi)=0$, that is, $(-1)^{w} \sigma_{w}(\xi)=c$. This is the coefficient of $x^{n-w}$ in $P(x)$. Hence $\left[x^{n-w}\right] P(x)=c$ with $P(x)$ irreducible of degree $n$ over $\mathbb{F}_{q}$.

Note the delta functions also satisfy the property that

$$
\delta_{w}\left(\left(a_{0}, \ldots, a_{n-1}\right)_{q}\right)=\delta_{w}\left(\left(a_{\rho(0)}, \ldots, a_{\rho(n-1)}\right)_{q}\right)
$$

for every permutation $\rho$ of the indices in $[0, n-1]$. In particular such functions have a natural well-studied dyadic analogue in the symmetric Boolean functions. These are Boolean functions $f: \mathbb{F}_{2}^{n} \rightarrow \mathbb{F}_{2}$ with the property that $f\left(x_{0}, \ldots, x_{n-1}\right)=$ $f\left(x_{\rho(0)}, \ldots, x_{\rho(n-1)}\right)$ for every permutation $\rho \in \mathcal{S}_{[0, n-1]}$; hence the value of $f\left(x_{0}, \ldots, x_{n-1}\right)$ 
depends only on the Hamming weight of $\left(x_{0}, \ldots, x_{n-1}\right)$. See for example [10,13] for some works on symmetric Boolean functions. Nevertheless in our case the domain of these $\delta_{w}$ functions is $\mathbb{Z}_{q^{n}-1}$ rather than $\mathbb{F}_{2}^{n}$. Although one may still represent the elements of $\mathbb{Z}_{q^{n}-1}$ as $n$-tuples, say by using the natural $q$-adic representation, the arithmetic here is not as nice as in $\mathbb{F}_{2}^{n}$. One has to consider the possibility that a "carry" may occur when adding or subtracting (this can make things quite chaotic) and also worry about reduction modulo $q^{n}-1$ (although this is much easier to deal with). These issues will come up again in the following section. The symmetry property in (5.2.1) of $\delta_{w}$ and of its convolutions will be exploited in the proof of Lemma 5.3.1 for the case when $(w, c)=(n / 2,0)$.

Before we move on to the following section, we need the fact asserted in Lemma 5.2.4 below. First for a permutation $\rho \in \mathcal{S}_{[0, n-1]}$ of the indices in the set $[0, n-1]$, define the map $\varphi_{\rho}: \mathbb{Z}_{q^{n}-1} \rightarrow \mathbb{Z}_{q^{n}-1}$ by

$$
\varphi_{\rho}\left(\left(a_{0}, \ldots, a_{n-1}\right)_{q}\right)=\left(a_{\rho(0)}, \ldots, a_{\rho(n-1)}\right)_{q}
$$

Note $\varphi_{\rho}$ is a permutation of $\mathbb{Z}_{q^{n}-1}$ with inverse $\varphi_{\rho}^{-1}=\varphi_{\rho^{-1}}$, for each $\rho \in \mathcal{S}_{[0, n-1]}$. For $k \in \mathbb{Z}_{q^{n}-1}$, let $\epsilon_{i}(k), 0 \leq i \leq n-1$, denote the digit of $q^{i}$ in the $q$-adic form of its canonical representative. Thus $0 \leq \epsilon_{i}(k) \leq q-1$. For $a, b \in \mathbb{Z}_{q^{n}-1}$ with $a+b \neq 0$, it is clear that if $\epsilon_{i}(a)+\epsilon_{i}(b) \leq q-1$, then $\epsilon_{i}(a+b)=\epsilon_{i}(a)+\epsilon_{i}(b)$. One can also check, for any $a, b \in \mathbb{Z}_{q^{n-1}}$ such that $\epsilon_{i}(a)+\epsilon_{i}(b) \leq q-1$ holds for every $0 \leq i \leq n-1$, that $\varphi_{\rho}(a+b)=\varphi_{\rho}(a)+\varphi_{\rho}(b)$ for every $\rho \in \mathcal{S}_{[0, n-1]}$, regardless of whether $a+b=0$ or not. By induction, $\varphi_{\rho}\left(a_{1}+\cdots+a_{s}\right)=\varphi_{\rho}\left(a_{1}\right)+\cdots+\varphi_{\rho}\left(a_{s}\right)$, whenever $a_{1}, \ldots, a_{s} \in \mathbb{Z}_{q^{n}-1}$ satisfy $\epsilon_{i}\left(a_{1}\right)+\cdots+\epsilon_{i}\left(a_{s}\right) \leq q-1$ for every $0 \leq i \leq n-1$.

Definition 5.2.3 (q-symmetric). For a function $f$ on $\mathbb{Z}_{q^{n}-1}$, we say that $f$ is $q$ symmetric if for all $a=\left(a_{0}, \ldots, a_{n-1}\right)_{q} \in \mathbb{Z}_{q^{n}-1}$ and all permutations $\rho \in \mathcal{S}_{[0, n-1]}$, we 
have $f\left(\varphi_{\rho}(a)\right)=f(a)$; that is,

$$
f\left(\left(a_{\rho(0)}, \ldots, a_{\rho(n-1)}\right)_{q}\right)=f\left(\left(a_{0}, \ldots, a_{n-1}\right)_{q}\right) .
$$

Note the $\delta_{w}$ functions are $q$-symmetric. Because $\epsilon_{i}(m) \leq 1$ for each $m \in \operatorname{supp}\left(\delta_{w}\right)=$ $\Omega(w)$ and each $0 \leq i \leq n-1$, it follows from the following lemma that the convolution of at most $q-1$ delta functions is also $q$-symmetric.

Lemma 5.2.4. Let $R$ be a ring and let $f_{1}, \ldots, f_{s}: \mathbb{Z}_{q^{n}-1} \rightarrow R$ be q-symmetric functions such that for each $a_{k} \in \operatorname{supp}\left(f_{k}\right), 1 \leq k \leq s$, we have $\epsilon_{i}\left(a_{1}\right)+\cdots+\epsilon_{i}\left(a_{s}\right) \leq$ $q-1$ for every $0 \leq i \leq n-1$. Then $f_{1} \otimes \cdots \otimes f_{s}$ is q-symmetric.

Proof. Recall the assumption on the supports imply that $\varphi_{\tau}\left(a_{1}+\cdots+a_{s}\right)=\varphi_{\tau}\left(a_{1}\right)+$ $\cdots+\varphi_{\tau}\left(a_{s}\right)$ for any $a_{k} \in \operatorname{supp}\left(f_{k}\right), 1 \leq k \leq s$, and any $\tau \in \mathcal{S}_{[0, n-1]}$. Since each $f_{k}$ is $q$-symmetric, $1 \leq k \leq s$, then $f_{k}(a)=f_{k}\left(\varphi_{\tau}(a)\right)$ for every $a \in \mathbb{Z}_{q^{n}-1}$. In particular $a \in \operatorname{supp}\left(f_{k}\right)$ if and only if $\varphi_{\tau}(a) \in \operatorname{supp}\left(f_{k}\right)$; hence $\varphi_{\tau}\left(\operatorname{supp}\left(f_{k}\right)\right)=\operatorname{supp}\left(f_{k}\right)$. Now let $m \in \mathbb{Z}_{q^{n}-1}$ and let $\rho \in \mathcal{S}_{[0, n-1]}$. Then it follows from the aforementioned observations 
that

$$
\begin{aligned}
& \left(f_{1} \otimes \cdots \otimes f_{s}\right)\left(\varphi_{\rho}(m)\right)=\sum_{\substack{j_{1}+\cdots+j_{s}=\varphi_{\rho}(m) \\
j_{1}, \ldots, j_{s} \in \mathbb{Z}_{q^{n}-1}}} f_{1}\left(j_{1}\right) \cdots f_{s}\left(j_{s}\right) \\
& =\sum_{\substack{j_{1}+\cdots+j_{s}=\varphi_{\rho}(m) \\
j_{1} \in \operatorname{supp}\left(f_{1}\right), \ldots, j_{s} \in \operatorname{supp}\left(f_{s}\right)}} f_{1}\left(j_{1}\right) \cdots f_{s}\left(j_{s}\right) \\
& =\sum_{\substack{\varphi_{\rho}-1 \\
j_{1} \in \operatorname{supp}\left(j_{1}+\cdots+j_{s}\right), \ldots, j_{s} \in \operatorname{supp}\left(f_{s}\right)}} f_{1}\left(j_{1}\right) \cdots f_{s}\left(j_{s}\right) \\
& =\sum_{\substack{\varphi_{\rho}-1\left(j_{1}\right)+\cdots+\varphi_{\rho^{-1}}\left(j_{s}\right)=m \\
j_{1} \in \operatorname{supp}\left(f_{1}\right), \ldots, j_{s} \in \operatorname{supp}\left(f_{s}\right)}} f_{1}\left(j_{1}\right) \cdots f_{s}\left(j_{s}\right) \\
& =\sum_{\substack{j_{1}+\cdots+j_{s}=m \\
\varphi_{\rho}\left(j_{1}\right) \in \operatorname{supp}\left(f_{1}\right), \ldots, \varphi_{\rho}\left(j_{s}\right) \in \operatorname{supp}\left(f_{s}\right)}} f_{1}\left(\varphi_{\rho}\left(j_{1}\right)\right) \cdots f_{s}\left(\varphi_{\rho}\left(j_{s}\right)\right) \\
& =\sum_{j_{1}+\cdots+j_{s}=m} f_{1}\left(j_{1}\right) \cdots f_{s}\left(j_{s}\right) \\
& \varphi_{\rho}\left(j_{1}\right) \in \operatorname{supp}\left(f_{1}\right), \ldots, \varphi_{\rho}\left(j_{s}\right) \in \operatorname{supp}\left(f_{s}\right) \\
& =\sum_{\substack{j_{1}+\cdots+j_{s}=m \\
j_{1} \in \varphi_{\rho}-1\left(\operatorname{supp}\left(f_{1}\right)\right), \ldots, j_{s} \in \varphi_{\rho}-1}} f_{1}\left(j_{1}\right) \cdots f_{s}\left(j_{s}\right) \\
& =\sum_{\substack{j_{1}+\cdots+j_{s}=m \\
j_{1} \in \operatorname{supp}\left(f_{1}\right), \ldots, j_{s} \in \operatorname{supp}\left(f_{s}\right)}} f_{1}\left(j_{1}\right) \cdots f_{s}\left(j_{s}\right) \\
& =\sum_{\substack{j_{1}+\cdots+j_{s}=m \\
j_{1}, \ldots, j_{s} \in \mathbb{Z}_{q^{n}-1}}} f_{1}\left(j_{1}\right) \cdots f_{s}\left(j_{s}\right) \\
& =\left(f_{1} \otimes \cdots \otimes f_{s}\right)(m),
\end{aligned}
$$

as required. 


\subsection{Least period of $\Delta_{w, c}$ and proof of Theorem 1.0.11}

In this section we prove in Lemma 5.3.1 that the least period of the $\Delta_{w, c}$ function of Lemma 5.2.2 is not a divisor of $\left(q^{n}-1\right) / \Phi_{n}(q)$, at least in the cases which suffice for a proof of Theorem 1.0.11. We note the proof of Lemma 5.3.1 is of a rather elementary and constructive type nature. There we treat the cases when $c \neq 0$ and $c=0$ separately. We remark that a version of the case when $c \neq 0$ and its combinatorial argument was given in [28]. For the sake of completeness, for the case when $c \neq 0$, we include a proof in the same spirit (see Case 1 of the proof that follows).

First for an integer $k=\sum_{i=0}^{\infty} \epsilon_{i}(k) q^{i}$, we let $s_{q}(k)=\sum_{i=0}^{\infty} \epsilon_{i}(k)$ denote the sum of the $q$-digits of $k$.

Lemma 5.3.1. Let $q$ be a power of a prime, let $n \geq 2$, let $w$ be an integer with $1 \leq w<n$, and let $c \in \mathbb{F}_{q}$. If $c=0$ and $n=2$, assume that $q$ is odd. If $c \neq 0$, further assume that $w<n-1$. Then the least period $r$ of $\Delta_{w, c}$ is not a divisor of $\left(q^{n}-1\right) / \Phi_{n}(q)$.

Proof. First, by the binomial theorem for convolution,

$$
\begin{aligned}
\left((-1)^{w} \delta_{w}-c \delta_{0}\right)^{\otimes(q-1)} & =\sum_{s=0}^{q-1}\left(\begin{array}{c}
q-1 \\
s
\end{array}\right)(-c)^{q-1-s}(-1)^{w s} \delta_{w}^{\otimes s} \\
& =\sum_{s=0}^{q-1}\left(\begin{array}{c}
q-1 \\
s
\end{array}\right)\left((-1)^{w+1} c\right)^{-s} \delta_{w}^{\otimes s}
\end{aligned}
$$

Hence

$$
\begin{aligned}
\Delta_{w, c} & :=\delta_{0}-\left((-1)^{w} \delta_{w}-c \delta_{0}\right)^{\otimes(q-1)} \\
& =-\sum_{s=1}^{q-1}\left(\begin{array}{c}
q-1 \\
s
\end{array}\right)\left((-1)^{w+1} c\right)^{-s} \delta_{w}^{\otimes s} .
\end{aligned}
$$

By Lucas' theorem, none of the binomial coefficients above are 0 modulo $p$, where $p$ 
is the characteristic of $\mathbb{F}_{q}$. Now note for any $m \in \mathbb{Z}_{q^{n}-1}$ that $\delta_{w}^{\otimes s}(m)$ is the number, modulo $p$, of ways to write $m$ as a sum of $s$ ordered values in $\Omega(w)$. Next we treat the cases when $c \neq 0$ and $c=0$ separately.

Case $1(c \neq 0)$ : Here we closely follow the combinatorial argument given in [28]. Now it is known that $m \in \operatorname{supp}\left(\Delta_{w, c}\right)$ if and only if there exists a unique $1 \leq s \leq$ $q-1$ such that $m \in \operatorname{supp}\left(\delta_{w}^{\otimes s}\right)$. In particular if $m \in \operatorname{supp}\left(\Delta_{w, c}\right)$, then $s_{q}\left(m \bmod \left(q^{n}-\right.\right.$ $1)) \leq(q-1) w$. This implies that $\operatorname{supp}\left(\Delta_{w, c}\right) \cap\left\{q^{n}-1, q^{n}-2, \ldots, q^{n}-q\right\}=\emptyset$ if $w<n-1$.

By Lemma 5.2.4, $\Delta_{w, c}$ is $q$-symmetric. For each $m \in \operatorname{supp}\left(\Delta_{w, c}\right)$ and each permutation $\rho \in \mathcal{S}_{[0, n-1]}$, we have $\Delta_{w, c}\left(\varphi_{\rho}(m)\right)=\Delta_{w, c}(m)$ and thus $\varphi_{\rho}(m) \in \operatorname{supp}\left(\Delta_{w, c}\right)$. If $r \mid\left(q^{n}-1\right) / \Phi_{n}(q)$, then $\operatorname{supp}\left(\Delta_{w, c}\right)$ is closed under addition and subtraction of $\left(q^{n}-1\right) / \Phi_{n}(q)$ modulo $q^{n}-1$, namely, $\Delta_{w, c}(m+k r)=\Delta_{w, c}(m)$ implies that if $m \in \operatorname{supp}\left(\Delta_{w, c}\right)$ then $m+k r \in \operatorname{supp}\left(\Delta_{w, c}\right)$.

In the following, we prove our statement by contradiction. Namely, if $r \mid\left(q^{n}-\right.$ $1) / \Phi_{n}(q)$ then we can find an element in $\operatorname{supp}\left(\Delta_{w, c}\right)$, which is greater than or equal to $q^{n}-q$.

For $n \neq 2,6$, we must have $\left(q^{n}-1\right) / r \geq \Phi_{n}(q)>q^{2}$. If $1<\epsilon_{n-1}(m), \epsilon_{n-2}(m)<q-$ 1 , we can add multiples of $r$ to $m$ so that $m^{\prime}=m+k r$ satisfies that $\epsilon_{n-1}\left(m^{\prime}\right)=\epsilon_{n-1}(m)$ and $\epsilon_{n-2}\left(m^{\prime}\right)=\epsilon_{n-1}(m)+1$. Permuting the highest two significant positions (say $\sigma$ ) and subtracting $k r$, we obtain another element $m^{\prime \prime}=\sigma\left(m^{\prime}\right)-k r \in \operatorname{supp}\left(\Delta_{w, c}\right)$ such that $\epsilon_{n-1}\left(m^{\prime \prime}\right)=\epsilon_{n-2}(m)+1$ and $\epsilon_{n-2}\left(m^{\prime \prime}\right)=\epsilon_{n-1}(m)-1$. That is, we can find an element $m^{\prime} \in \operatorname{supp}\left(\Delta_{w, c}\right)$ such that $m^{\prime}-m=q^{n-1}-q^{n-2}$. Continue doing this, we can find an element, say $m$ abusing notation, in $\operatorname{supp}\left(\Delta_{w, c}\right)$ such that one of $\epsilon_{n-1}(m)$ and $\epsilon_{n-2}(m)$ is 0 or $q-1$.

For each $m \in \operatorname{supp}\left(\Delta_{w, c}\right)$, we can find a sequence of permutations so that any two digits $\epsilon_{i}(m)$ and $\epsilon_{j}(m)$ of $m$ could be moved to the highest significant positions. 
This means that we have another element $m^{\prime}$ in $\operatorname{supp}\left(\Delta_{w, c}\right)$ so that $\epsilon_{n-1}\left(m^{\prime}\right)=\epsilon_{i}(m)$, $\epsilon_{n-2}\left(m^{\prime}\right)=\epsilon_{j}(m)$ and $\epsilon_{k}\left(m^{\prime}\right)(0 \leq k \leq n-3)$ must be one of $\epsilon_{t}(m)$ such that $t \neq i, j$.

Hence, repeating the above procedures, we can find another element, say $m$, in $\operatorname{supp}\left(\Delta_{w, c}\right)$ such that all digits except at most one equal to 0 or $q-1$. Without loss of generality, we assume $\epsilon_{n-1}(m)=a$. Because $w<n-1$, we have at least one $j$ such that $\epsilon_{j}(m)=0$. Again, without loss of generality, we assume that $\epsilon_{n-2}(m)=0$. If $a \neq 0$, applying the same procedure as above, we can obtain an element $m^{\prime} \in$ $\operatorname{supp}\left(\Delta_{w, c}\right)$ such that $\epsilon_{n-2}\left(m^{\prime}\right)=q-1$ and $\epsilon_{n-1}\left(m^{\prime}\right)=a-1$. Similarly, we can find an element in $\operatorname{supp}\left(\Delta_{w, c}\right)$ such that all digits equal to $q-1$ except $\epsilon_{0}$. This contradicts to $\operatorname{supp}\left(\Delta_{w, c}\right) \cap\left\{q^{n}-1, q^{n}-2, \ldots, q^{n}-q\right\}=\emptyset$.

If $a=0$, then all digits of $m$ are either 0 or $q-1$. Applying the above procedure on $\epsilon_{n-1}(m)=q-1$ and $\epsilon_{n-2}(m)=0$, we can obtain $m^{\prime} \in \operatorname{supp}\left(\Delta_{w, c}\right)$ such that $\epsilon_{n-1}\left(m^{\prime}\right)=$ $q-2$ and $\epsilon_{n-2}\left(m^{\prime}\right)=q-1$. Then we always turn other 0 digits into $q-1$ digit. Eventually we have an element in the $\operatorname{supp}\left(\Delta_{w, c}\right)$ such that all digits except the least significant digit are $q-1$, this contradicts to $\operatorname{supp}\left(\Delta_{w, c}\right) \cap\left\{q^{n}-1, q^{n}-2, \ldots, q^{n}-q\right\}=\emptyset$.

For $n=2, r \mid q-1$. If $m \in \operatorname{supp}\left(\Delta_{w, c}\right)$ then there exists a $k$ such that $m+k(q-1) \in$ $\operatorname{supp}\left(\Delta_{w, c}\right)$ and $m+k(q-1)>q^{2}-q$, a contradiction.

For $n=6,\left(q^{n}-1\right) / \Phi_{n}(q)=q^{4}+q^{3}-q-1$. If $m$ contains at most three $q-1$ digits in its q-ary expansion, then $\epsilon_{n-2}\left(m+\left(q^{n}-1\right) / \Phi_{n}(q)\right)=\epsilon_{n-2}(m)+1$. Permuting the highest significant two digits of $m+\left(q^{n}-1\right) / \Phi_{n}(q)$ and subtract $\left(q^{n}-1\right) / \Phi_{n}(q)$ from it, we obtain an element $m^{\prime} \in \operatorname{supp}\left(\Delta_{w, c}\right)$ such that $\epsilon_{n-1}\left(m^{\prime}\right)=\epsilon_{n-2}(m)+1$ and $\epsilon_{n-2}\left(m^{\prime}\right)=\epsilon_{n-1}(m)-1$. In this way, we can find an element in $\operatorname{supp}\left(\Delta_{w, c}\right)$ with four $q-1$ digits. Without loss of generality, we can assume that $m \in \operatorname{supp}\left(\Delta_{w, c}\right)$ such that $\epsilon_{n-1}(m)=q-1$ and $\epsilon_{2}(m)=\epsilon_{1}(m)=\epsilon_{0}(m)=q-1$, namely, $m$ has the $q$-ary representation $q-1, a, b, q-1, q-1, q-1$. Subtracting $\left(q^{n}-1\right) / \Phi_{n}(q)$ from $m$, we obtain an element in the support such that its q-ary representation is $q-1, a-1, b, 0,1,0$. 
Permuting the highest two significant digits and adding $\left(q^{n}-1\right) / \Phi_{n}(q)$ back, we obtain $m^{\prime} \in \operatorname{supp}\left(\Delta_{w, c}\right)$ such that $m^{\prime}$ has $q$-ary representation $a, 0, b, q-1, q-1, q-1$. However, $m^{\prime}$ and $m$ have different weights, a contradiction.

Case $2(c=0)$ : Assume $c=0$. Note $\Delta_{w, 0}=\delta_{0}-\delta_{w}^{\otimes(q-1)}$ and $\Delta_{w, 0}(0)=1$. Thus $\Delta_{w, 0}(r)=\Delta_{w, 0}(0+r)=1$. Since $0<r<q^{n}-1$, necessarily $\delta_{w}^{\otimes(q-1)}(r)=-1$. In particular $r \in \operatorname{supp}\left(\delta_{w}^{\otimes(q-1)}\right)$ and $s_{q}(r)=(q-1) w$. Because $1 \leq r<q^{n}-1$ is a period of $\Delta_{w, 0}$, so is $r^{\prime}=q^{n}-1-r$ with $1 \leq r^{\prime}<q^{n}-1$. Then the previous arguments similarly imply that $s_{q}\left(r^{\prime}\right)=(q-1) w$. Given that $s_{q}\left(r^{\prime}\right)=(q-1) n-s_{q}(r)$, it follows $w=n / 2$ and $s_{q}(r)=(q-1) n / 2$. In particular $n$ is even and $\Delta_{w, 0}=\Delta_{n / 2,0}=\delta_{0}-\delta_{n / 2}^{\otimes(q-1)}$.

Consider the case when $n>2$ : Suppose not all digits of $r$ are the same (since $s_{q}(r)=(q-1) n / 2$, it is equivalent to supposing that $r \neq\left(q^{n}-1\right) / 2$; this is the case in particular when $q$ is even). Clearly either there exists $k \in[0, n-2]$ such that $r_{k}>r_{k+1}$ or the sequence $r_{0}, \ldots, r_{n-1}$ is non-decreasing. Suppose the former holds. Fix any such $k$ and let $\sigma$ be the permutation of $[0, n-1]$ which fixes each index in $[0, n-1] \backslash\{k, k+1\}$ and maps $k \mapsto k+1$ and $k+1 \mapsto k$. Thus

$$
\varphi_{\sigma}(r)=r_{k} q^{k+1}+r_{k+1} q^{k}+\sum_{i \in[0, n-1] \backslash\{k, k+1\}} r_{i} q^{i}>r_{k+1} q^{k+1}+r_{k} q^{k}+\sum_{i \in[0, n-1] \backslash\{k, k+1\}} r_{i} q^{i}=r
$$

since $r_{k}>r_{k+1}$. Because $\varphi_{\sigma}(r)$ is obtained via a permutation of the digits of $r$, and $0<r<q^{n}-1$, then $0<\varphi_{\sigma}(r)<q^{n}-1$. Now note

$$
\begin{aligned}
\varphi_{\sigma}(r)-r & =\left(r_{k}-r_{k+1}\right) q^{k+1}-\left(r_{k}-r_{k+1}\right) q^{k} \\
& =\left(r_{k}-r_{k+1}-1\right) q^{k+1}+\left(q-\left(r_{k}-r_{k+1}\right)\right) q^{k} .
\end{aligned}
$$

Since $1 \leq r_{k}-r_{k+1} \leq q-1$, it follows the above coefficients are contained in the set 
$[0, q-1]$; hence this is the $q$-adic form of $\varphi_{\sigma}(r)-r$ and one can see that $s_{q}\left(\varphi_{\sigma}(r)-r\right)=$ $q-1$.

Because $\delta_{n / 2}$ is $q$-symmetric with $\epsilon_{i}(m) \leq 1$ for each $m \in \operatorname{supp}\left(\delta_{n / 2}\right)=\Omega(n / 2)$ and each $0 \leq i \leq n-1$, it follows from Lemma 5.2.4 that $\delta_{n / 2}^{\otimes(q-1)}$ is $q$-symmetric. In particular $\delta_{n / 2}^{\otimes(q-1)}\left(\varphi_{\sigma}(r)\right)=\delta_{n / 2}^{\otimes(q-1)}(r)$. Since $\varphi_{\sigma}(r) \neq 0$, then $\Delta_{n / 2,0}\left(\varphi_{\sigma}(r)\right)=$ $-\delta_{n / 2}^{\otimes(q-1)}\left(\varphi_{\sigma}(r)\right)=-\delta_{n / 2}^{\otimes(q-1)}(r)=1$; hence $\varphi_{\sigma}(r) \in \operatorname{supp}\left(\Delta_{n / 2,0}\right)$. Given that $\Delta_{n / 2,0}$ is $r$-periodic, then $\varphi_{\sigma}(r)-r \in \operatorname{supp}\left(\Delta_{n / 2,0}\right)$. Since $0<\varphi_{\sigma}(r)-r<q^{n}-1$, then $\varphi_{\sigma}(r)-r \in \operatorname{supp}\left(\delta_{n / 2}^{\otimes(q-1)}\right)$. It follows $s_{q}\left(\varphi_{\sigma}(r)-r\right)=(q-1) n / 2$, contradicting $s_{q}\left(\varphi_{\sigma}(r)-r\right)=q-1$ with $n>2$. Necessarily the $q$-digits $r_{0}, \ldots, r_{n-1}$ of $r$ must form a non-decreasing sequence. Since not all digits of $r$ are the same, in particular $r_{n-1}>r_{0}$.

Since $\Delta_{n / 2,0}$ is $r$-periodic, it is $r^{\prime \prime}:=\left(q r \bmod \left(q^{n}-1\right)\right)$-periodic. Note $0<r^{\prime \prime}<q^{n}-$ 1 and $r^{\prime \prime}=\left(r_{n-1}, r_{0}, r_{1}, \ldots, r_{n-2}\right)_{q}$. However observe that $r_{0}=\epsilon_{1}\left(r^{\prime \prime}\right)<\epsilon_{0}\left(r^{\prime \prime}\right)=r_{n-1}$. Then we can reproduce the previous arguments with $r$ and $k$ substituted with $r^{\prime \prime}$ and 0 , respectively, to obtain a contradiction. Thus for $n>2$, it is impossible that $\Delta_{n / 2,0}$ is $r$-periodic if $0<r<q^{n}-1$ and not all digits of $r$ are the same. In particular when $q$ is even and $n>2, \Delta_{n / 2,0}$ must have maximum least period $q^{n}-1$.

Note that at this point we have proved that: If $w \neq n / 2$, or $n>2$ and $q$ is even with $w=n / 2$, then $r=q^{n}-1$. In the case when $q$ odd with $n>2$ and $w=n / 2$, we have shown that either $r=\left(q^{n}-1\right) / 2$ (all digits of $r$ are the same) or no such $r$ with $0<r<q^{n}-1$ can be a period of $\Delta_{n / 2,0}$ (when not all digits of $r$ are the same), whence the least period of $\Delta_{n / 2,0}$ must be the maximum, $q^{n}-1$.

Consider now the case with $n=2$ and $q$ odd: Here $w=n / 2=1$ and we claim that $r>q-1$. On the contrary, suppose $r \leq q-1$. Since $s_{q}(r)=(q-1) n / 2=q-1$, it follows that $r=q-1$. Note there is exactly one way to write $r=q-1$ as a sum of $q-1$ ordered elements in $\Omega(1)=\{1, q\}$, namely as $q-1=1+\cdots+1$, a total of 
$q-1$ times. Thus $\delta_{1}^{\otimes(q-1)}(r)=1$. This contradicts the fact (see the beginning of the proof of Case 2) that $\delta_{1}^{\otimes(q-1)}(r)=-1$ with $q$ odd. Hence the claim follows.

It remains to notice that the least period $r$ of $\Delta_{w, c}$ satisfies $r>\left(q^{n}-1\right) / \Phi_{n}(q)$ in every case here, and hence $r \nmid\left(q^{n}-1\right) / \Phi_{n}(q)$. Indeed, this follows immediately from the fact that $\Phi_{n}(q)>q-1$ for $n \geq 2$. In the case of $n=2$ with $w=1$, we have $r>q-1=\left(q^{2}-1\right) /(q+1)=\left(q^{2}-1\right) / \Phi_{2}(q)$ as well. This concludes the proof of Lemma 5.3.1.

Proof of Theorem 1.0.11. It is elementary to show that every element of $\mathbb{F}_{q}^{*}$ is the norm of an element of degree $n$ over $\mathbb{F}_{q}$; see for example [37]. Thus we may assume $w<n$. If $w=n-1$, we claim that the number of monic irreducible polynomials with prescribed coefficient of $x$ is positive; indeed, by Carlitz's result [12], there exists an irreducible polynomial with both arbitrarily prescribed trace and nonzero norm coefficients, and then the claim follows by taking the appropriate monic reciprocal. The other cases follow from Lemma 5.3.1 together with Lemma 5.2.2. 


\section{Chapter 6}

\section{On the number of $N$-free elements}

\section{with prescribed trace}

In this chapter ${ }^{1}$ we study the number of $N$-free elements, in an extension $\mathbb{F}_{q^{m}}$ of $\mathbb{F}_{q}$, with prescribed trace in $\mathbb{F}_{q}$. We first briefly recall the definition of $N$-free: For a positive divisor $N$ of $q^{m}-1$, we say that a non-zero element $\xi \in \mathbb{F}_{q^{m}}^{*}$ is $N$-free if, for any $d \mid N, \xi=\gamma^{d}, \gamma \in \mathbb{F}_{q^{m}}$, implies $d=1$. Equivalently, $\xi$ is $N$-free if and only if $\xi=\alpha^{k}$ for some integer $k$ that is coprime to $N$. Note that the definition of $N$-free is independent of the choice of the primitive element $\alpha$.

The content of this chapter is as follows. We start off in Section 6.1 by deriving a formula for $Z_{q, m, N}(c)$ in terms of Gaussian periods (see Lemma 6.1.4). In Section 6.2 we specifically consider the case of the zero trace and simplify our formula, in Subsection 6.2.1, with the use of a lemma due to Ding and Yang [26]. Then in Subsection 6.2.2 we prove Corollaries 1.0.23 and 1.0.25. In Section 6.3 we give a

\footnotetext{
${ }^{1}$ This chapter is substantively based on the following article originally published in Journal of Number Theory, https://doi.org/10.1016/j.jnt.2015.09.008. It is released under a Creative Commons Attribution Non-Commercial No Derivatives License.

[69] A. Tuxanidy and Q. Wang, On the number of $N$-free elements with prescribed trace, Journal of Number Theory, v.160 (2016), p.536-565. https://doi.org/10.1016/j.jnt.2015.09.008
} 
sufficient criteria for uniformity to occur (see Theorem 1.0.26), as well as some other related results. Then in Section 6.4 we focus our attention to the number $P_{q, m, N}(c)$ and give some other related results. In particular we obtain that for $p$ a Mersenne prime, the number of non-zero elements in $\mathbb{F}_{p^{4}}^{*}$ with the corresponding large order $2(p+1)\left(p^{2}+1\right)$ and having absolute trace zero, is $2 \phi\left(p^{2}+1\right)$ (see Theorem 6.4.5). Finally in Appendix we include a table of data corresponding to Corollary 1.0.25, giving the number of primitive elements in quartic extensions of Mersenne prime fields, with absolute trace zero, for the first ten Mersenne primes.

\subsection{A formula for $Z_{q, m, N}$}

In this section we derive, in terms of Gaussian periods, a formula for the number of $N$ free elements with prescribed trace (see Lemma 6.1.4). Note that Cohen and Prešern [20] already did so in terms of Gaussian sums (see their Lemma 2.2). However by the means of Gaussian periods we will be able to apply Ding-Yang lemmas (Lemma 2.3.1 and 2.3.2) thus obtaining, for the case of the zero trace, the simplified version of Theorem 1.0.18 and the fact that $Z_{q, m, N}(0)=\Theta(K) Z_{q, m, \operatorname{gcd}(Q, N)}(0)$ already mentioned in the introduction. Recall that here $K$ is the part of $N$ that is coprime to $Q$, and $\Theta(K)=\phi(K) / K$.

The following characteristic function for $N$-free elements, due to Vinogradov, is typically used in works on the topic. See for instance $[16,17,20,21]$ and the references therein.

Proposition 6.1.1 (Vinogradov). Let $N$ be a positive divisor of $q^{m}-1$ and let 
$\xi \in \mathbb{F}_{q^{m}}^{*}$. Then

$$
\frac{\phi(N)}{N} \sum_{d \mid N} \frac{\mu(d)}{\phi(d)} \sum_{\operatorname{ord}(\psi)=d} \psi(\xi)= \begin{cases}1 & \text { if } \xi \text { is } N \text {-free } \\ 0 & \text { otherwise }\end{cases}
$$

where in the inner sum $\psi$ runs through all the multiplicative characters of $\mathbb{F}_{q^{m}}$ with order d.

We will however consider the following apparently simpler form of the characteristic function.

Lemma 6.1.2. Let $N$ be a positive divisor of $q^{m}-1$ and let $\xi \in \mathbb{F}_{q^{m}}^{*}$. For each positive divisor d of $N$, fix a multiplicative character $\psi_{d}$ of $\mathbb{F}_{q^{m}}$ with order $d$. Then

$$
\sum_{d \mid N} \frac{\mu(d)}{d} \sum_{j=0}^{d-1} \psi_{d}^{j}(\xi)= \begin{cases}1 & \text { if } \xi \text { is } N \text {-free } \\ 0 & \text { otherwise }\end{cases}
$$

Proof. Let $\alpha$ be primitive in $\mathbb{F}_{q^{m}}$. Then $\xi=\alpha^{k}$ for some integer $k$. Note that

$$
\frac{1}{d} \sum_{j=0}^{d-1} e^{2 \pi \sqrt{-1} j k / d}= \begin{cases}1 & \text { if } d \mid k \\ 0 & \text { otherwise }\end{cases}
$$

Hence

$$
\sum_{d \mid N} \frac{\mu(d)}{d} \sum_{j=0}^{d-1} \psi_{d}^{j}\left(\alpha^{k}\right)=\sum_{d \mid N} \frac{\mu(d)}{d} \sum_{j=0}^{d-1} e^{2 \pi \sqrt{-1} j k / d}=\sum_{d \mid \operatorname{gcd}(N, k)} \mu(d)= \begin{cases}1 & \text { if } \operatorname{gcd}(N, k)=1 \\ 0 & \text { otherwise. }\end{cases}
$$


Recall that for a positive divisor $N$ of $q^{m}-1$, an integer $k$, and $c \in \mathbb{F}_{q}$, we denote

$$
\Delta_{k}(N)=\sum_{d \mid N} \mu(d) \eta_{k}^{\left(d, q^{m}\right)}
$$

The following proposition highlights some of the basic properties of $\Delta_{k}$.

Proposition 6.1.3. Let $N \mid q^{m}-1$ and let $k \in \mathbb{Z}$. Then we have the following three identities:

$$
\begin{aligned}
\Delta_{N k}(N) & =\Delta_{0}(N) ; \\
\sum_{i=0}^{N-1} \Delta_{i}(N) & =-\phi(N) ; \text { and } \\
\Delta_{k}(N) & =\sum_{\substack{i=1 \\
\operatorname{gcd}(N, i-k)=1}}^{q^{m}-1} \chi_{q^{m}}\left(\alpha^{i}\right) .
\end{aligned}
$$

Proof. The first identity follows from the fact that the sequence of periods of type $\left(N, q^{m}\right)$ has period $N$, i.e., $\eta_{k}^{\left(N, q^{m}\right)}=\eta_{0}^{\left(N, q^{m}\right)}$ whenever $k \equiv 0(\bmod N)$. To prove the second identity, note that for each positive divisor $d$ of $N$,

$$
\sum_{i=0}^{N-1} \eta_{i}^{\left(d, q^{m}\right)}=\frac{N}{d} \sum_{i=0}^{d-1} \eta_{i}^{\left(d, q^{m}\right)}=\frac{N}{d} \sum_{x \in \mathbb{F}_{q^{m}}^{*}} \chi_{q^{m}}(x)=-\frac{N}{d} .
$$

Hence

$$
\sum_{i=0}^{N-1} \Delta_{i}(N)=\sum_{d \mid N} \mu(d) \sum_{i=0}^{N-1} \eta_{i}^{\left(d, q^{m}\right)}=-\sum_{d \mid N} \mu(d) \frac{N}{d}=-\phi(N) .
$$


For the last identity, by (2.3.2) and Lemma 6.1.2, we have

$$
\begin{aligned}
\Delta_{k}(N) & =\sum_{d \mid N} \mu(d) \eta_{k}^{\left(d, q^{m}\right)}=\sum_{d \mid N} \frac{\mu(d)}{d} \sum_{j=0}^{d-1} \overline{\psi_{d}^{j}\left(\alpha^{k}\right)} G_{q^{m}}\left(\psi_{d}^{j}\right) \\
& =\sum_{d \mid N} \frac{\mu(d)}{d} \sum_{j=0}^{d-1} \overline{\psi_{d}^{j}\left(\alpha^{k}\right)} \sum_{i=1}^{q^{m}-1} \chi_{q^{m}}\left(\alpha^{i}\right) \psi_{d}^{j}\left(\alpha^{i}\right) \\
& =\sum_{i=1}^{q^{m}-1} \chi_{q^{m}}\left(\alpha^{i}\right) \sum_{d \mid N} \frac{\mu(d)}{d} \sum_{j=0}^{d-1} \psi_{d}^{j}\left(\alpha^{i-k}\right) \\
& =\sum_{\substack{i=1 \\
\operatorname{gcd}(N, i-k)=1}}^{q^{m}-1} \chi_{q^{m}}\left(\alpha^{i}\right) .
\end{aligned}
$$

For the sake of brevity let us also fix the following notation for the remaining of the chapter.

$$
f_{k}(N, c, \Delta):=\sum_{i=0}^{q-2} \overline{\chi_{q}\left(\alpha^{Q i} c\right)} \Delta_{Q i+k}(N) .
$$

Now we give the general formula for $Z_{q, m, N}(c)$.

Lemma 6.1.4. Let $N$ be a positive divisor of $q^{m}-1$ and let $c$ be an arbitrary element of $\mathbb{F}_{q}$. Then

$$
Z_{q, m, N}(c)=\frac{1}{q}\left(\frac{q^{m}-1}{N} \phi(N)+f_{0}(N, c, \Delta)\right)
$$

Proof. By the orthogonality relation in (2.3.1) and by the transitivity of the trace 
function, note that

$\frac{1}{q} \sum_{a \in \mathbb{F}_{q}} \overline{\chi_{q}(a c)} \chi_{q^{m}}\left(a \alpha^{k}\right)=\frac{1}{q} \sum_{a \in \mathbb{F}_{q}} \chi_{q}\left(a\left(\operatorname{Tr}_{\mathbb{F}_{q^{m}} / \mathbb{F}_{q}}\left(\alpha^{k}\right)-c\right)\right)= \begin{cases}1 & \text { if } \operatorname{Tr}_{\mathbb{F}_{q^{m}} / \mathbb{F}_{q}}\left(\alpha^{k}\right)=c \\ 0 & \text { otherwise. }\end{cases}$

Thus if we multiply the characteristic function above with that of Lemma 6.1.2, and then sum over all the elements in $\mathbb{F}_{q^{m}}^{*}$, we get

$$
\begin{aligned}
Z_{q, m, N}(c) & =\frac{1}{q} \sum_{d \mid N} \mu(d) \sum_{a \in \mathbb{F}_{q}} \overline{\chi_{q}(a c)} \frac{1}{d} \sum_{j=0}^{d-1} \sum_{k=1}^{q^{m}-1} \chi_{q^{m}}\left(a \alpha^{k}\right) \psi_{d}^{j}\left(\alpha^{k}\right) \\
& =\frac{1}{q}\left(\sum_{k=1}^{q^{m}-1} \sum_{d \mid N} \frac{\mu(d)}{d} \sum_{j=0}^{d-1} \psi_{d}^{j}\left(\alpha^{k}\right)+\sum_{i=0}^{q-2} \overline{\chi_{q}\left(\alpha^{Q i} c\right)} \sum_{d \mid N} \mu(d) \frac{1}{d} \sum_{j=0}^{d-1} \sum_{x \in \mathbb{F}_{q^{m}}^{*}} \chi_{q^{m}}\left(\alpha^{Q i} x\right) \psi_{d}^{j}(x)\right) \\
& =\frac{1}{q}\left(\frac{q^{m}-1}{N} \phi(N)+\sum_{i=0}^{q-2} \overline{\chi_{q}\left(\alpha^{Q i} c\right)} \sum_{d \mid N} \mu(d) \eta_{Q i}^{\left(d, q^{m}\right)}\right) \\
& =\frac{1}{q}\left(\frac{q^{m}-1}{N} \phi(N)+f_{0}(N, c, \Delta)\right) .
\end{aligned}
$$

\subsection{The case of the zero trace}

In this section we consider the special case of the zero trace and prove some of the corresponding assertions already mentioned in the introduction, and give some other related results. We start off in Subsection 6.2.1 by deriving Theorem 1.0.18 and giving some immediate consequences. See Corolleries 1.0.19, 1.0.20, 1.0.21 and 1.0.22. Then in Subsection 6.2.2 we prove Corollaries 1.0.23 and 1.0.25, and also prove the "semiprimitive" characterization in Proposition 1.0.24. 


\subsubsection{Simplification of $Z_{q, m, N}(0)$ and direct consequences}

First, in the case of the zero trace, we apply the Ding-Yang lemmas (Lemma 2.3.1 and 2.3.2) to simplify the expression for $f_{k}(N, 0, \Delta)$.

Lemma 6.2.1. Let $N \mid q^{m}-1, k \in \mathbb{Z}$ and $K_{Q}$ be the largest divisor of $N$ that is coprime to $Q$. Then

$$
f_{k}(N, 0, \Delta)=(q-1) \frac{\phi\left(K_{Q}\right)}{K_{Q}} \Delta_{k}(\operatorname{gcd}(Q, N))
$$

Proof. For a positive divisor $d$ of $q^{m}-1$, let us denote, for the sake of brevity, $g(d)=\operatorname{gcd}(Q, d)$. Now, by Lemma 2.3.2,

$$
\begin{aligned}
f_{k}(N, 0, \Delta) & =\sum_{d \mid N} \mu(d) \sum_{i=0}^{q-2} \eta_{Q i+k}^{\left(d, q^{m}\right)} \\
& =(q-1) \sum_{d \mid N} \mu(d) \frac{g(d)}{d} \eta_{k}^{\left(g(d), q^{m}\right)} .
\end{aligned}
$$

Note $\operatorname{Rad}(N)=\operatorname{Rad}\left(K_{Q}\right) \operatorname{Rad}(\operatorname{gcd}(Q, N))$ is the product of the two coprime numbers $\operatorname{Rad}\left(K_{Q}\right)$ and $\operatorname{Rad}(\operatorname{gcd}(Q, N))$. Then we can write any positive divisor $d$ of $\operatorname{Rad}(N)$ uniquely as $d=y z$, where $y \mid \operatorname{Rad}\left(K_{Q}\right)$ and $z \mid \operatorname{Rad}(\operatorname{gcd}(Q, N))$. Moreover $g(y z)=z$ for any such $y, z$. Hence

$$
\begin{aligned}
f_{k}(N, 0, \Delta) & =(q-1) \sum_{y \mid K_{Q}} \sum_{z \mid \operatorname{gcd}(Q, N)} \mu(y z) \frac{g(y z)}{y z} \eta_{k}^{\left(g(y z), q^{m}\right)} \\
& =(q-1) \sum_{y \mid K_{Q}} \frac{\mu(y)}{y} \sum_{z \mid \operatorname{gcd}(Q, N)} \mu(z) \eta_{k}^{\left(z, q^{m}\right)} \\
& =(q-1) \frac{\phi\left(K_{Q}\right)}{K_{Q}} \Delta_{k}(\operatorname{gcd}(Q, N)) .
\end{aligned}
$$


Proof of Theorem 1.0.18. By Euler's product formula for $\phi$, and using the fact that $K_{Q}$ is coprime to $\operatorname{gcd}(Q, N)$ with $\operatorname{Rad}(N)=\operatorname{Rad}\left(K_{Q}\right) \operatorname{Rad}(\operatorname{gcd}(Q, N))$, we have

$$
\begin{aligned}
\frac{\phi(N)}{N} & =\prod_{\ell \mid N}\left(1-\frac{1}{\ell}\right) \\
& =\left(\prod_{\ell \mid K_{Q}}\left(1-\frac{1}{\ell}\right)\right)\left(\prod_{\ell \mid \operatorname{gcd}(Q, N)}\left(1-\frac{1}{\ell}\right)\right) \\
& =\frac{\phi\left(K_{Q}\right)}{K_{Q}} \frac{\phi(\operatorname{gcd}(Q, N))}{\operatorname{gcd}(Q, N)},
\end{aligned}
$$

where in the three products $\ell$ runs through all the distinct prime divisors of $N, K_{Q}$ and $\operatorname{gcd}(Q, N)$, respectively. Hence by Lemmas 6.1.4 and 6.2.1,

$$
\begin{aligned}
Z_{q, m, N}(0) & =\frac{1}{q}\left(\left(q^{m}-1\right) \frac{\phi(N)}{N}+(q-1) \frac{\phi\left(K_{Q}\right)}{K_{Q}} \Delta_{0}(\operatorname{gcd}(Q, N))\right) \\
& =\frac{\phi\left(K_{Q}\right)}{q K_{Q}}\left(\left(q^{m}-1\right) \frac{\phi(\operatorname{gcd}(Q, N))}{\operatorname{gcd}(Q, N)}+(q-1) \Delta_{0}(\operatorname{gcd}(Q, N))\right) \\
& =\frac{(q-1) \phi\left(K_{Q}\right)}{q K_{Q}}\left(\frac{Q}{\operatorname{gcd}(Q, N)} \phi(\operatorname{gcd}(Q, N))+\Delta_{0}(\operatorname{gcd}(Q, N))\right) .
\end{aligned}
$$

In particular one obtains in Lemma 6.2.2 the number of primitives with zero trace. The second equality (on the right) gives Lemma 2.1 in [20].

Lemma 6.2.2. Let $D$ be the smallest positive divisor of $q-1$ such that $(q-1) / D$ is coprime to $Q$. Then the number of primitive elements $\xi$ in $\mathbb{F}_{q^{m}}$ with $\operatorname{Tr}_{\mathbb{F}_{q^{m}} / \mathbb{F}_{q}}(\xi)=0$ 
is given by

$$
\begin{aligned}
Z_{q, m, q^{m}-1}(0) & =D \phi\left(\frac{q-1}{D}\right) \frac{\phi(Q)+\Delta_{0}(Q)}{q} \\
& =D \phi\left(\frac{q-1}{D}\right) \frac{Z_{q, m, Q}(0)}{q-1} .
\end{aligned}
$$

We now derive some other immediate consequences to Theorem 1.0.18.

Proof of Corollary 1.0.19. Follows directly from Theorem 1.0.18 and the fact that $\Delta_{0}(1)=-1$.

Lemma 6.2.3. Let $q$ be a power of a prime $p$ and assume that $Q=\left(q^{\ell}-1\right) /(q-1)$ is prime for some prime $\ell$. Then

$$
\Delta_{0}(Q)= \begin{cases}0 & \text { if } \ell \neq p \\ -q & \text { otherwise }\end{cases}
$$

Proof. Since $Q$ is prime, then $\Delta_{0}(Q)=-1-\eta_{0}^{\left(Q, q^{\ell}\right)}$. Now the result follows from Lemma 2.3.7.

Proof of Corollary 1.0.20. Note that $\operatorname{gcd}(q-1, Q)=1$ since otherwise $Q \mid q-1$ contradicting $Q>q-1$ for $\ell \geq 2$. Now the result follows directly from Lemmas 6.2.3 and 6.2.2.

Proof of Corollary 1.0.21. Follows directly from Theorem 1.0.18 and Lemma 2.3.4.

Proof of Corollary 1.0.22. Note that since $\operatorname{gcd}(Q, N)=3$, then $Q \equiv m \equiv 0$ (mod 3). The result now follows from Lemma 2.3.5 and Theorem 1.0.18. 


\subsubsection{Proof of Corollaries 1.0.23 and 1.0.25}

In this subsection we prove Corollaries 1.0.23 and 1.0.25, corresponding to the case of the zero trace. We employ the known explicit formulas for the Gaussian periods in the semi-primitive case (see Lemma 2.3.6) to first derive, in the following lemma, the value of the sum $\Delta_{0}(N)$ for $N$ falling under the category of Lemma 2.3.6. Then by Theorem 1.0.18 we get the result of Corollary 1.0.23. One of course can then naturally consider whether this result applies to primitives, but unfortunately, as Proposition 1.0.24 shows, it only extends to primitives in quartic extensions of Mersenne prime fields. Mersenne primes also appear in the trivial case for which a formula is known. This is the case when all the irreducibles are also the primitives, that is, when $q=2$ and $m=\ell$ with $2^{\ell}-1$ being a Mersenne prime. See the comments under the statement of Theorem 1.0.16.

Lemma 6.2.4. Let $s m$ be even with $m>1$, let $q=p^{s}$ be a power of a prime $p$, and suppose that $n>1$ is not a power of 2 and satisfies $n \mid q^{m}-1$. Further assume there exists a positive integer $j$ such that $p^{j} \equiv-1(\bmod \ell)$ for every prime divisor $\ell \geq 3$

of $n$, and that $j$ is the least such. Define $\gamma=s m / 2 j$ and let $\eta_{0}^{\left(2, q^{m}\right)}$ be as in Lemma 2.3.4.

(a) If $\gamma$ and $p$ are odd, $n$ is even and $\left(p^{j}+1\right) / 2$ is odd, then

$$
\Delta_{0}(n)=-\eta_{0}^{\left(2, q^{m}\right)}-\left(1+q^{m / 2}\right)\left(\frac{1}{2}+\frac{\phi(n)}{n}\right)
$$

(b) In all other cases,

$$
\Delta_{0}(n)=\epsilon_{2} \cdot\left(\frac{(-1)^{\gamma+1} q^{m / 2}-1}{2}-\eta_{0}^{\left(2, q^{m}\right)}\right)+\frac{(-1)^{\gamma} q^{m / 2}-1}{n} \phi(n),
$$


where

$$
\epsilon_{2}= \begin{cases}1 & \text { if } n \text { is even } \\ 0 & \text { otherwise }\end{cases}
$$

Proof. First note the assumption on $n$ and $j$ means that, for every positive $3 \leq d \mid$ $\operatorname{Rad}(n)$, we have that $j$ is the least such that $p^{j} \equiv-1(\bmod d)$.

(a) Consider any such $d$ as above. If $d$ is odd, then $\left(p^{j}+1\right) / d$ is even and so $\eta_{0}^{\left(d, q^{m}\right)}$ belongs to case (b) of Lemma 2.3.6. Moreover since 2 has multiplicity 1 in the factorization of $p^{j}+1$, then $\left(p^{j}+1\right) / 2 d$ is odd if so is $d$; in this case $\eta_{0}^{\left(2 d, q^{m}\right)}$ belongs to case (a) in Lemma 2.3.6. Now let $V_{2}$ be the largest power of 2 dividing $n$. We then have

$$
\begin{aligned}
\sum_{3 \leq d \mid n} \mu(d) \eta_{0}^{\left(d, q^{m}\right)} & =\sum_{\substack{3 \leq d \mid n \\
d \text { odd }}}\left(\mu(d) \eta_{0}^{\left(d, q^{m}\right)}+\mu(2 d) \eta_{0}^{\left(2 d, q^{m}\right)}\right)=\sum_{\substack{3 \leq d \mid n \\
d \text { odd }}} \mu(d)\left(\eta_{0}^{\left(d, q^{m}\right)}-\eta_{0}^{\left(2 d, q^{m}\right)}\right) \\
& =\sum_{\substack{3 \leq d \mid n \\
d \text { odd }}} \mu(d)\left(\frac{(d-1) q^{m / 2}-1}{d}+\frac{q^{m / 2}+1}{2 d}\right)=\sum_{\substack{3 \leq d \mid n \\
d \text { odd }}} \mu(d)\left(q^{m / 2}-\frac{q^{m / 2}+1}{2 d}\right) \\
& =\frac{1-q^{m / 2}}{2}+\sum_{\substack{d \mid n \\
d \text { odd }}} \mu(d)\left(q^{m / 2}-\frac{q^{m / 2}+1}{2 d}\right) \\
& =\frac{1-q^{m / 2}}{2}+\sum_{\substack{d \mid n / V_{2} \\
2}(d)\left(q^{m / 2}-\frac{q^{m / 2}+1}{2 d}\right)} \\
& =\frac{1-q^{m / 2}}{2}-\frac{q^{m / 2}+1}{2} \sum_{d \mid n / V_{2}} \frac{\mu(d)}{d}=\frac{1-q^{m / 2}}{2}-\frac{\left(1+q^{m / 2}\right) V_{2}}{2 n} \phi\left(\frac{n}{V_{2}}\right) \\
& =\frac{1-q^{m / 2}}{2}-\frac{1+q^{m / 2}}{n} \phi(n)
\end{aligned}
$$

since $V_{2}$ is coprime to $n / V_{2}$ and $\phi\left(V_{2}\right)=V_{2} / 2$. Then we have

$$
\Delta_{0}(n)=-1-\eta_{0}^{\left(2, q^{m}\right)}+\sum_{3 \leq d \mid n} \mu(d) \eta_{0}^{\left(d, q^{m}\right)}=-1-\eta_{0}^{\left(2, q^{m}\right)}+\frac{1-q^{m / 2}}{2}-\frac{1+q^{m / 2}}{n} \phi(n)
$$


Hence the result follows.

(b) As before assume $d \mid n$ with $d \geq 3$ and $\mu(d) \neq 0$. We claim that $\eta_{0}^{\left(d, q^{m}\right)}$ belongs to case (b) in Lemma 2.3.6. Indeed, if $p$ or $\gamma$ is even, then $\eta_{0}^{\left(d, q^{m}\right)}$ belongs to $(b)$. If $d$ is odd, necessarily $\left(p^{j}+1\right) / d$ is even unless $p$ is even; hence (b). This also takes care of the case when $n$ is odd. Finally if $n$ is even and 2 has multiplicity greater than 1 in the factorization of $p^{j}+1$, then $\left(p^{j}+1\right) / 2 d$ is even for any such $d$ odd. The claim follows. Hence by Lemma 2.3.6 (b) we have

$$
\begin{aligned}
\sum_{3 \leq d \mid n} \mu(d) \eta_{0}^{(d, r)} & =\sum_{3 \leq d \mid n} \mu(d)\left(\frac{(-1)^{\gamma+1} d q^{m / 2}+(-1)^{\gamma} q^{m / 2}-1}{d}\right) \\
& =1+\epsilon_{2} \cdot\left(\frac{(-1)^{\gamma+1} q^{m / 2}-1}{2}\right)+\sum_{d \mid n} \mu(d)\left((-1)^{\gamma+1} q^{m / 2}+\frac{(-1)^{\gamma} q^{m / 2}-1}{d}\right) \\
& =1+\epsilon_{2} \cdot\left(\frac{(-1)^{\gamma+1} q^{m / 2}-1}{2}\right)+\sum_{d \mid n} \mu(d)\left(\frac{(-1)^{\gamma} q^{m / 2}-1}{d}\right) \\
& =1+\epsilon_{2} \cdot\left(\frac{(-1)^{\gamma+1} q^{m / 2}-1}{2}\right)+\frac{(-1)^{\gamma} q^{m / 2}-1}{n} \sum_{d \mid n} \mu(d) \frac{n}{d} \\
& =1+\epsilon_{2} \cdot\left(\frac{(-1)^{\gamma+1} q^{m / 2}-1}{2}\right)+\frac{(-1)^{\gamma} q^{m / 2}-1}{n} \phi(n) .
\end{aligned}
$$

Hence the result follows.

Proof of Corollary 1.0.23. It follows directly from Lemma 6.2.4 and Theorem 1.0 .18 .

In order to prove Proposition 1.0.24 we make use of Mihăilescu's breakthrough result [51], also known as Catalan's conjecture. Although like Wiles' Theorem (Fermat's Last Theorem) it is easily stated, it took 160 years for the conjecture to be finally solved, by Mihăilescu [51].

Theorem 6.2.5 (Mihăilescu (2004)). Let $x, y, a, b \in \mathbb{N}$ with $a, b>1$. If $x^{a}-y^{b}=1$, then $x=b=3$ and $y=a=2$. 
For a positive integer $k$, we let $v_{2}(k)$ denote the multiplicity of 2 in the factorization of $k$. For an integer $b$ coprime to $k$, we let $\operatorname{ord}_{k}(b)$ denote the multiplicative order of $b$ modulo $k$.

Proof of Proposition 1.0.24. Let $q=p^{s}$ be the power of a prime $p$, with $s \geq 1$. The case when $m=2$ is clear as $\left(q^{2}-1\right) /(q-1)=q+1$ and so we can let $j=s$. We may thus suppose $m>2$. Now assume that the congruence is satisfied, i.e., that

$$
p^{j} \equiv-1 \quad\left(\bmod \operatorname{Rad}\left(\frac{p^{s m}-1}{p^{s}-1}\right)\right)
$$

holds.

We claim that $m$ is a power of 2 . On the contrary, suppose the odd part, $k$, of $m$, satisfies $k>1$. Let $t$ be the odd part of $s k$. Then $t>1$ and $(s k, t)=t \nmid s$ since $k>1$ and $k \mid t$. Since $t \mid s k$, we have $\left(p^{s k}-1, p^{t}-1\right)=p^{t}-1>1$. Moreover $d:=\operatorname{Rad}\left(\left(\frac{p^{s k}-1}{p^{s}-1}, p^{t}-1\right)\right)>1$ since otherwise $\left(p^{t}-1\right) \mid\left(p^{s}-1\right)$ contrary to $t \nmid s$. Because $k>1$ is odd, then both $\frac{p^{s k}-1}{p^{s}-1}$ and hence $d$ are odd. In particular $d \geq 3$.

Note that $p^{t} \equiv 1(\bmod d)$. Thus if $a$ is the smallest positive integer such that $p^{a} \equiv 1(\bmod d)$, then $a \mid t$ and hence $a$ is odd. Now observe that $p^{j} \equiv-1(\bmod d)$, since $d\left|\operatorname{Rad}\left(\frac{p^{s k}-1}{p^{s}-1}\right)\right| \operatorname{Rad}\left(\frac{p^{s m}-1}{p^{s}-1}\right)$. Thus $p^{2 j} \equiv 1(\bmod d)$. It follows that $a \nmid j$ but $a \mid 2 j$. This however implies that $a$ is even, a contradiction. The claim follows. Hence $m=2^{n}$ for some $n \geq 2$ and

$$
\frac{p^{s m}-1}{p^{s}-1}=\prod_{i=0}^{n-1}\left(p^{s 2^{i}}+1\right)
$$

Since $n \geq 2$, we can let $i \geq 0$ be arbitrary such that both $p^{s 2^{i}}+1$ and $p^{s 2^{i+1}}+1$ divide $\left(p^{s 2^{n}}-1\right) /\left(p^{s}-1\right)$. Suppose there exist square-free divisors $d_{i}, d_{i+1} \geq 3$ of $p^{s 2^{i}}+1$ and $p^{s 2^{i+1}}+1$, respectively. For the sake of brevity let $a_{i}:=\operatorname{ord}_{d_{i}}(p)$ and 
$a_{i+1}:=\operatorname{ord}_{d_{i+1}}(p)$. Since $p^{j} \equiv-1\left(\bmod d_{i}\right)$, we have $a_{i} \nmid j$ but $a_{i} \mid 2 j$ implying that $v_{2}(j)=v_{2}\left(a_{i}\right)-1$. Similarly since $p^{s 2^{i}} \equiv-1\left(\bmod d_{i}\right)$, then $a_{i} \nmid s 2^{i}$ but $a_{i} \mid 2\left(s 2^{i}\right)$. This implies that $v_{2}\left(a_{i}\right)=v_{2}(s)+i+1$. It then follows that $v_{2}(j)=v_{2}(s)+i$. Working with $d_{i+1}$ and $a_{i+1}$ now we similarly deduce that $v_{2}(j)=v_{2}(s)+i+1$, a contradiction. Necessarily at least one of $p^{s 2^{i}}+1, p^{s 2^{i+1}}+1$, is a power of 2 . But note that, for any positive integer $b$, we have $p^{b}+1=2^{v}$ if and only if $p^{b}=2^{v}-1$. In this case it is clear that $v>1$; then we can not have $b>1$ since otherwise Theorem 6.2.5 is contradicted. In particular $p$ is a Mersenne prime. Moreover $s 2^{i}=1$ or $s 2^{i+1}=1$. Of these two, only $s 2^{i}=1$ is possible, whence $i=0, s=1$, and $q=p$ is a Mersenne prime. Because $i \geq 0$ was arbitrary such that both $p^{s 2^{i}}+1$ and $p^{s 2^{i+1}}+1$ divide $\left(p^{s 2^{n}}-1\right) /\left(p^{s}-1\right)$, necessarily $n=2$ is the only possibility, whence $m=4$ and $\left(p^{s m}-1\right) /\left(p^{s}-1\right)=(p+1)\left(p^{2}+1\right)$.

Since $p$ is a Mersenne prime, $p+1$ is a power of 2 and $2 \mid\left(p^{2}+1\right)$ imply that $\operatorname{Rad}\left((p+1)\left(p^{2}+1\right)\right)=\operatorname{Rad}\left(p^{2}+1\right)$. Thus it only remains to show that $\operatorname{Rad}\left(p^{2}+1\right) \mid$ $\left(p^{j}+1\right)$ if and only if $j=2 k$ for some odd $k \geq 1$. Assume that $\operatorname{Rad}\left(p^{2}+1\right) \mid\left(p^{j}+1\right)$. First it is easy to check that $p^{2}+1$ is not a power of 2 . Hence we can let $d \geq 3$ with $d \mid \operatorname{Rad}\left(p^{2}+1\right)$. Then $p^{2} \equiv-1(\bmod d)$ and $p^{4} \equiv 1(\bmod d) \operatorname{implies} \operatorname{ord}_{d}(p)=4$. Since $d\left|\operatorname{Rad}\left(p^{2}+1\right)\right|\left(p^{j}+1\right)$, then $p^{j} \equiv-1(\bmod d)$ implying $4 \nmid j$ but $4 \mid 2 j$. This means that $j=2 k$ for some odd $k \geq 1$. Conversely let $j=2 k$ for any odd $k \geq 1$. Note that $p^{2 k}=\left(p^{2}\right)^{k}=\left[\left(p^{2}+1\right)-1\right]^{k}$. Then by the Binomial Theorem we have

$$
p^{2 k}=\left[\left(p^{2}+1\right)-1\right]^{k}=\sum_{i=0}^{k}\left(\begin{array}{c}
k \\
i
\end{array}\right)\left(p^{2}+1\right)^{i}(-1)^{k-i}=-1+\sum_{i=1}^{k}\left(\begin{array}{c}
k \\
i
\end{array}\right)\left(p^{2}+1\right)^{i}(-1)^{k-i} .
$$

Hence

$$
p^{2 k}+1=\sum_{i=1}^{k}\left(\begin{array}{c}
k \\
i
\end{array}\right)\left(p^{2}+1\right)^{i}(-1)^{k-i}
$$

and so $\left(p^{2}+1\right) \mid\left(p^{2 k}+1\right)$. Consequently $\operatorname{Rad}\left(p^{2}+1\right) \mid\left(p^{2 k}+1\right)$. 
Proof of Corollary 1.0.25 . By Proposition 1.0.24 we have that $Q=\left(p^{4}-1\right) /(p-$ $1)=(p+1)\left(p^{2}+1\right)$ satisfies $p^{j} \equiv-1(\bmod \operatorname{Rad}(Q))$ with $j=2 k$ for every odd $k \geq 1$; in particular for $j=2$. If $\ell \geq 3$ is a prime divisor of $Q$, clearly $\ell \nmid p+1$ (since $p+1$ is a power of 2$)$. Then $j=2$ is the least such that $p^{j} \equiv-1(\bmod \ell)$. Corollary 1.0.23 then applies with $s=1, m=4, N=p^{4}-1, j=2, n=Q=(p+1)\left(p^{2}+1\right)$, and $\gamma=1$. With the notation of Corollary 1.0.23, it is easy to show that $K_{Q}=(p-1) / 2$. Since $p, \gamma$, are odd while $n$ is even and 2 has multiplicity 1 in the factorization of $p^{2}+1$, then case (a) of Lemma 6.2.4 applies. In this case we get, using the fact that $p \equiv 3(\bmod 4)$ together with Lemma 2.3.4,

$$
\begin{aligned}
\Delta_{0}\left(p^{2}+1\right) & =\Delta_{0}\left((p+1)\left(p^{2}+1\right)\right)=-1+\frac{1+p^{2}}{2}+\frac{1-p^{2}}{2}-\frac{1}{p+1} \phi\left((p+1)\left(p^{2}+1\right)\right) \\
& =-\frac{1}{p+1} \phi\left((p+1)\left(p^{2}+1\right)\right)=-\phi\left(p^{2}+1\right)=-\phi\left(\frac{p^{2}+1}{2}\right) .
\end{aligned}
$$

It then follows from Lemma 6.2.2 that

$$
Z_{p, 4, p^{4}-1}(0)=\frac{1}{p}\left(\phi\left(p^{4}-1\right)-2 \phi\left(\frac{p-1}{2}\right) \phi\left(\frac{p^{2}+1}{2}\right)\right) .
$$

It remains to note that

$$
\phi\left(\frac{p-1}{2}\right) \phi\left(\frac{p^{2}+1}{2}\right)=\phi\left(\frac{(p-1)\left(p^{2}+1\right)}{4}\right)=\frac{1}{2} \phi\left(\frac{p^{4}-1}{p+1}\right)
$$

since

$$
\operatorname{gcd}\left(\frac{p-1}{2}, \frac{p^{2}+1}{2}\right)=\operatorname{gcd}\left(4, \frac{(p-1)\left(p^{2}+1\right)}{4}\right)=1
$$

and $p^{4}-1=(p-1)(p+1)\left(p^{2}+1\right)$. The result follows. 


\subsection{Uniformity in the case of the non-zero trace}

In this section we explore the concept of uniformity, already discussed at the start of the chapter. That is, the main concern here is as follows: what triples $(q, m, N)$, with $N \mid q^{m}-1$, are such that $Z_{q, m, N}(c)$ is constant for every non-zero $c \in \mathbb{F}_{q}^{*}$ ? Accordingly, in this section we prove Theorem 1.0.26. As a consequence of this and of Corollary 1.0.23, Corollary 1.0.27 is straightforward. In particular, in the case of primitives, i.e., $N=q^{m}-1$, we give sufficient conditions for $Z_{q, m, q^{m}-1}(c)$ to behave uniformly for $c \in \mathbb{F}_{q}^{*}$. See Corollary 6.3.3 for this.

Lemma 6.3.1. Let $N \mid q^{m}-1, c \in \mathbb{F}_{q}^{*}$ be arbitrary, $K_{Q}$ be the largest divisor of $N$ that is coprime to $Q$. Then

$$
\begin{aligned}
q Z_{q, m, N}(c \neq 0)= & \frac{q^{m}-1}{N} \phi(N)+K_{Q} \frac{\frac{q^{m}-1}{N} \phi(N)-q Z_{q, m, N}(0)}{(q-1) \phi\left(K_{Q}\right)}+f_{0}(N, c, \Delta) \\
& -f_{0}(\operatorname{gcd}(Q, N), c, \Delta) .
\end{aligned}
$$

Proof. By Proposition 6.1 .3 and using the fact that $\sum_{a \in \mathbb{F}_{q}^{*}} \overline{\chi_{q}(a c)}=-1$ for $c \neq 0$, we get

$$
\begin{aligned}
f_{0}(\operatorname{gcd}(Q, N), c, \Delta) & =\sum_{i=0}^{q-2} \overline{\chi_{q}\left(\alpha^{Q i} c\right)} \Delta_{Q i}(\operatorname{gcd}(Q, N))=\sum_{i=0}^{q-2} \overline{\chi_{q}\left(\alpha^{Q i} c\right)} \Delta_{0}(\operatorname{gcd}(Q, N)) \\
& =-\Delta_{0}(\operatorname{gcd}(Q, N)) .
\end{aligned}
$$

Hence

$$
f_{0}(N, c, \Delta)=-\Delta_{0}(\operatorname{gcd}(Q, N))+f_{0}(N, c, \Delta)-f_{0}(\operatorname{gcd}(Q, N), c, \Delta) .
$$

By Theorem 1.0.18 we can write $\Delta_{0}(\operatorname{gcd}(Q, N))$ in terms of $Z_{q, m, N}(0)$. Now the 
expression for $Z_{q, m, N}(c \neq 0)$ follows from Lemma 6.1.4.

Proof of Theorem 1.0.26. If every prime divisor of $N$ divides $Q$, then $\operatorname{Rad}(N)=$ $\operatorname{Rad}(\operatorname{gcd}(Q, N))$ and $K_{Q}=1$. Now the result follows from Lemma 6.3.1 together with the fact that $f_{0}(d, c, \Delta)=f_{0}(\operatorname{Rad}(d), c, \Delta)$ for every $d \mid q^{m}-1$.

Lemma 6.3.2. Let $b, m>1$. Then $\operatorname{Rad}\left(b^{m}-1\right)=\operatorname{Rad}\left(\frac{b^{m}-1}{b-1}\right)$ if and only if every prime factor of $b-1$ divides $m$.

Proof. First note that $\operatorname{Rad}\left(b^{m}-1\right)=\operatorname{Rad}\left(\frac{b^{m}-1}{b-1}\right)$ if and only if $\operatorname{Rad}(b-1)$ $\operatorname{Rad}\left(\frac{b^{m}-1}{b-1}\right)$. Now

$$
\begin{aligned}
\frac{b^{m}-1}{b-1}= & 1+b+b^{2}+\cdots+b^{m-1} \\
= & (1-b)+(b-1)+\left(b^{2}-1\right)+\cdots+\left(b^{m-1}-1\right) \\
& +b+m-1 .
\end{aligned}
$$

It follows that $\operatorname{Rad}(b-1) \mid \operatorname{Rad}\left(\frac{b^{m}-1}{b-1}\right)$ if and only if $\operatorname{Rad}(b-1)$ divides $m$.

As a consequence of Lemma 6.3.2, we obtain the following immediate result.

Corollary 6.3.3. Let $q$ be a power of a prime and $m \in \mathbb{N}$. If every prime factor of $q-1$ divides $m$, then, for every element $c \in \mathbb{F}_{q}^{*}$, the number, $Z_{q, m, q^{m}-1}(c)$, of primitive elements $\xi \in \mathbb{F}_{q^{m}}$ with $\operatorname{Tr}_{\mathbb{F}_{q}^{m} / \mathbb{F}_{q}}(\xi)=c$, is given by

$$
Z_{q, m, q^{m}-1}(c \neq 0)=\frac{\phi\left(q^{m}-1\right)-Z_{q, m, q^{m}-1}(0)}{q-1}
$$

Some other consequences to Theorem 1.0.26 are the following. 
Corollary 6.3.4. Let $N \mid q^{m}-1$ such that $\operatorname{Rad}(N) \mid Q$ and let $\mathbb{F}_{t}$ be any subfield of $\mathbb{F}_{q}$. Then, for all $c_{t} \in \mathbb{F}_{t}^{*}$ and $c_{q} \in \mathbb{F}_{q}^{*}$, we have

$$
Z_{t, m\left[\mathbb{F}_{q}: \mathbb{F}_{t}\right], N}\left(c_{t}\right)=\frac{\frac{q^{m}-1}{N} \phi(N)-Z_{t, m\left[\mathbb{F}_{q}: \mathbb{F}_{t}\right], N}(0)}{t-1}=\frac{q}{t} Z_{q, m, N}\left(c_{q}\right)
$$

Proof. Follows from Theorem 1.0.26 together with the fact that, since $N \mid\left(q^{m}-\right.$ $1) /(q-1)$ and $\left(q^{m}-1\right) /(q-1) \mid\left(q^{m}-1\right) /(t-1)$, then $N \mid\left(q^{m}-1\right) /(t-1)$.

Proof of Corollary 1.0.28. The first equality follows directly from Theorem 1.0.18 and Theorem 1.0.26 together with the fact mentioned in the proof of Corollary 6.3.4. The second equality follows from the first together with Corollary 6.3.4. Indeed,

$$
\begin{aligned}
q Z_{q, m, N}(0)-t Z_{t, m\left[\mathbb{F}_{q}: \mathbb{F}_{t}\right], N}(0) & =q\left(\Delta_{0}(N)+Z_{q, m, N}\left(c_{q}\right)\right)-t\left(\Delta_{0}(N)+Z_{t, m\left[\mathbb{F}_{q}: \mathbb{F}_{t}\right], N}(0)\right) \\
& =(q-t) \Delta_{0}(N)+q Z_{q, m, N}\left(c_{q}\right)-t Z_{t, m\left[\mathbb{F}_{q}: \mathbb{F}_{t}\right], N}(0) \\
& =(q-t) \Delta_{0}(N) .
\end{aligned}
$$

Corollary 6.3.5. Let $N \mid q^{m}-1$ such that $\operatorname{Rad}(N) \mid Q$. Then $Z_{t, m\left[\mathbb{F}_{q}: \mathbb{F}_{t}\right], N}(0)$ is related to $Z_{q, m, N}(0)$ by the equation

$$
(q-1) Z_{t, m\left[\mathbb{F}_{q}: \mathbb{F}_{t}\right], N}(0)=\left(q-\frac{q}{t}\right) Z_{q, m, N}(0)+\left(\frac{q}{t}-1\right) \frac{q^{m}-1}{N} \phi(N) .
$$

Proof. By Corollary 1.0.28 we have

$$
Z_{t, m\left[\mathbb{F}_{q}: \mathbb{F}_{t}\right], N}(0)-Z_{t, m\left[\mathbb{F}_{q}: \mathbb{F}_{t}\right], N}\left(c_{t}\right)=Z_{q, m, N}(0)-Z_{q, m, N}\left(c_{q}\right) .
$$


Hence, by Corollary 6.3.4,

$$
\begin{aligned}
Z_{t, m\left[\mathbb{F}_{q}: \mathbb{F}_{t}\right], N}(0) & =Z_{q, m, N}(0)+\left(\frac{q}{t}-1\right) Z_{q, m, N}\left(c_{q}\right) \\
& \left.=Z_{q, m, N}(0)\right)+\left(\frac{q}{t}-1\right)\left(\frac{\frac{q^{m}-1}{N} \phi(N)-Z_{q, m, N}(0)}{q-1}\right) \\
& =\frac{\left(q-\frac{q}{t}\right) Z_{q, m, N}(0)+\left(\frac{q}{t}-1\right) \frac{q^{m}-1}{N} \phi(N)}{q-1} .
\end{aligned}
$$

\subsection{Connection to $P_{q, m, N}(c)$}

In this section we briefly explore the seemingly related number, $P_{q, m, N}(c)$, of elements in $\mathbb{F}_{q^{m}}^{*}$ with order $N$ and with prescribed trace $c$. We start off by deriving a formula for the number of elements with order $N$ in an arbitrary subset $A$ of $\mathbb{F}_{q^{m}}$ and apply this to obtain a formula for $P_{q, m, N}(c)$ (see Lemma 6.4.1).

Let $L_{Q}$ be the largest divisor of $q^{m}-1$ with the same radical as that of $Q$. In the special case of the zero trace and the case when $L_{Q} \mid N$, we show in Lemma 6.4.3 the identity $Z_{q, m, N}(0)=\frac{q^{m}-1}{N} P_{q, m, N}(0)$. As a consequence of this and of Cohen's result (see Theorem 1.0.17 in the Introduction) we characterize the existence of elements of order $N$ (with $L_{Q} \mid N$ ) in $\mathbb{F}_{q^{m}}^{*}$ with trace 0 . Moreover from this and Corollary 1.0.25 we give in Corollary 6.4.5 the number of elements of order $2(p+1)\left(p^{2}+1\right)$ with absolute trace zero in quartic extensions of Mersenne prime fields $\mathbb{F}_{p}$.

For a subset $A \subseteq \mathbb{F}_{q^{m}}$ and a divisor $N$ of $q^{m}-1$, denote with $M_{q, m, N}(A)$ the number of non-zero elements in $A$ having multiplicative order $N$ in $\mathbb{F}_{q^{m}}^{*}$. In particular, $M_{q, m, q^{m}-1}(A)$ denotes the number of primitive elements of $\mathbb{F}_{q^{m}}$ that are contained in $A$. 
Lemma 6.4.1. Let $q$ be a prime power and $m$ be a positive integer. Let $A$ be a subset of $\mathbb{F}_{q^{m}}$ and $N$ be a positive divisor of $q^{m}-1$. Then the number of elements in $A$ that have multiplicative order $N$ is given by

$$
M_{q, m, N}(A)=\frac{1}{q^{m}-1} \sum_{d \mid N} \mu(d) \frac{N}{d}\left|\left\{x \in \mathbb{F}_{q^{m}}^{*}: x^{\frac{\left(q^{m}-1\right)}{N} d} \in A\right\}\right|
$$

In particular, for $c \in \mathbb{F}_{q}$, the number of elements $\beta \in \mathbb{F}_{q^{m}}^{*}$ with order $N$ and satisfying $\operatorname{Tr}_{\mathbb{F}_{q} m / \mathbb{F}_{q}}(\beta)=c$, is given by

$$
\begin{aligned}
P_{q, m, N}(c) & =\frac{N}{q\left(q^{m}-1\right)} \sum_{d \mid N} \frac{\mu(d)}{d} \sum_{a \in \mathbb{F}_{q}} \overline{\chi_{q}(a c)} \sum_{x \in \mathbb{F}_{q^{m}}^{*}} \chi_{q^{m}}\left(a x^{\frac{q^{m}-1}{N} d}\right) \\
& =\frac{1}{q}\left(\phi(N)+\sum_{i=0}^{q-2} \overline{\chi_{q}\left(\alpha^{Q i} c\right)} \sum_{d \mid N} \mu(d) \eta_{Q i}^{\left(\frac{q^{m}-1}{N} d, r\right)}\right) .
\end{aligned}
$$

Proof. If we define the arithmetic function $f(n):=\sum_{d \mid n} M_{q, m, d}(A)$, then by the Möbius inversion formula,

$$
M_{q, m, N}(A)=\sum_{d \mid N} \mu(d) f(N / d)=\sum_{d \mid N} \mu(d) \sum_{b \mid N / d} M_{q, m, b}(A) .
$$

Since $f(N / d)$ represents the number of elements in $A$ with orders that are divisors of $N / d$, and each such element can be written uniquely as $\alpha^{\frac{\left(q^{m}-1\right)}{N} d i}$ for $0 \leq i<N / d$, then

$$
\begin{aligned}
\sum_{b \mid N / d} M_{q, m, b}(A) & =\left|\left\{0 \leq i<N / d: \alpha^{\frac{\left(q^{m}-1\right)}{N} d i} \in A\right\}\right| \\
& =\frac{N}{d\left(q^{m}-1\right)}\left|\left\{x \in \mathbb{F}_{q^{m}}^{*}: x^{\frac{\left(q^{m}-1\right)}{N} d} \in A\right\}\right| .
\end{aligned}
$$

With regards to $P_{q, m, N}(c)$, observe that if we let $A_{c}:=\left\{x \in \mathbb{F}_{q^{m}}^{*}: \operatorname{Tr}_{\mathbb{F}_{q^{m}} / \mathbb{F}_{q}}(x)=\right.$ 
$c\}$, then $P_{q, m, N}(c)=M_{q, m, N}\left(A_{c}\right)$. Now it remains to obtain the expression for $\mid\{x \in$ $\left.\mathbb{F}_{q^{m}}^{*}: x^{\frac{q^{m}-1}{N} d} \in A_{c}\right\}|=|\left\{x \in \mathbb{F}_{q^{m}}^{*}: \operatorname{Tr}_{\mathbb{F}_{q} m / \mathbb{F}_{q}}\left(x^{\frac{q^{m}-1}{N} d}\right)=c\right\} \mid$, which can be done by applying (2.3.1). Indeed, by (2.3.1) and by the transitivity of the trace function,

$$
\begin{aligned}
\left|\left\{x \in \mathbb{F}_{q^{m}}^{*}: \operatorname{Tr}_{\mathbb{F}_{q} / \mathbb{F}_{q}}\left(x^{\frac{q^{m}-1}{N} d}\right)=c\right\}\right| & =\sum_{x \in \mathbb{F}_{q}^{*}} \frac{1}{q} \sum_{a \in \mathbb{F}_{q}} \chi_{q}\left(a\left(\operatorname{Tr}_{\mathbb{F}_{q} m / \mathbb{F}_{q}}\left(x^{\frac{q^{m}-1}{N} d}\right)-c\right)\right) \\
& =\frac{1}{q} \sum_{a \in \mathbb{F}_{q}} \overline{\chi_{q}(a c)} \sum_{x \in \mathbb{F}_{q}^{*}} \chi_{q}\left(\operatorname{Tr}_{\mathbb{F}_{q^{m}} / \mathbb{F}_{q}}\left(a x^{\frac{q^{m}-1}{N} d}\right)\right) \\
& =\frac{1}{q} \sum_{a \in \mathbb{F}_{q}} \overline{\chi_{q}(a c)} \sum_{x \in \mathbb{F}_{q}^{*}} \chi_{q^{m}}\left(a x^{\frac{q^{m}-1}{N} d}\right) .
\end{aligned}
$$

Hence the result follows.

For $k \in \mathbb{Z}$ and $N \mid q^{m}-1$, we denote

$$
\Gamma_{k}(N)=\sum_{d \mid N} \mu(d) \eta_{k}^{\left(\frac{q^{m}-1}{N} d, q^{m}\right)}
$$

For $\beta \in \mathbb{F}_{q^{m}}$, let $W_{H}(N, \beta)$ denote the Hamming weight of the $n$-tuple, where $n=$ $\left(q^{m}-1\right) / N$, given by

$$
c(N, \beta):=\left(\operatorname{Tr}_{\mathbb{F}_{q^{m}} / \mathbb{F}_{q}}(\beta), \operatorname{Tr}_{\mathbb{F}_{q^{m}} / \mathbb{F}_{q}}\left(\beta \alpha^{N}\right), \ldots, \operatorname{Tr}_{\mathbb{F}_{q} / \mathbb{F}_{q}}\left(\beta \alpha^{(n-1) N}\right)\right) .
$$

For an integer $t \neq 0$, let $\omega(t)$ be the number of distinct prime divisors of $t$.

The following proposition gives some general identities relating $Z_{q, m, N}(c), P_{q, m, N}(c)$, through the Möbius inversion formula, as well as shows their connection to Hamming weights.

Proposition 6.4.2. Let $q$ be a power of a prime, $m$ be a positive integer, $N \mid q^{m}-1$ 
and $k \in \mathbb{Z}$. Then the following identities hold:

$$
\begin{aligned}
\sum_{d \mid \operatorname{Rad}(N)} \mu(d) \Delta_{k}\left(\frac{\operatorname{Rad}(N)}{d}\right) & =(-1)^{\omega(N)} \eta_{k}^{\left(\operatorname{Rad}(N), q^{m}\right)} ; \\
\sum_{d \mid N} \Gamma_{k}(d) & =\eta_{k}^{\left(\frac{q^{m}-1}{N}, q^{m}\right)} ; \\
\sum_{d \mid \operatorname{Rad}(N)} \mu(d) \Delta_{k}\left(\frac{\operatorname{Rad}(N)}{d}\right) & =(-1)^{\omega(N)} \sum_{d \mid\left(\frac{q^{m}-1}{\operatorname{Rad}(N)}\right)} \Gamma_{k}(d) ; \\
(-1)^{\omega(N)} \sum_{d \mid \operatorname{Rad}(N)} \mu(d) f_{0}\left(\frac{\operatorname{Rad}(N)}{d}, \Delta, c\right) & =q \sum_{d \mid\left(\frac{q^{m}-1}{\operatorname{Rad}(N)}\right)} P_{q, m, d}(c)-\frac{q^{m}-1}{\operatorname{Rad}(N)} ; \\
f_{0}(N, \Delta, c) & =q \sum_{d \mid N} \mu(d) \sum_{b \mid\left(\frac{q^{m}-1}{d}\right)} P_{q, m, b}(c)-\frac{q^{m}-1}{N} \phi(N) ; \\
Z_{q, m, N}(c) & =\sum_{d \mid N} \mu(d) \sum_{b \mid\left(\frac{q^{m}-1}{d}\right)} P_{q, m, b}(c) ; \\
Z_{q, m, N}(0) & =\frac{q^{m}-1}{N} \phi(N)-\sum_{d \mid N} \mu(d) W_{H}(d, 1) .
\end{aligned}
$$

Proof. To prove the first six identities we use the Möbius inversion formula together with Lemma 6.1.4 and Lemma 6.4.1. The last identity follows from the one before it. Indeed, noting that

$$
W_{H}(d, 1)=\sum_{c \in \mathbb{F}_{q}^{*} b \mid\left(\frac{q^{m}-1}{d}\right)} P_{q, r, b}(c),
$$

we get

$$
\begin{aligned}
\frac{q^{m}-1}{N} \phi(N)-Z_{q, m, N}(0) & =\sum_{c \in \mathbb{F}_{q}^{*}} Z_{q, m, N}(c)=\sum_{d \mid N} \mu(d) \sum_{c \in \mathbb{F}_{q}^{*}} \sum_{b \mid\left(\frac{q^{m}-1}{d}\right)} P_{q, r, b}(c) \\
& =\sum_{d \mid N} \mu(d) W_{H}(d, 1) .
\end{aligned}
$$


We can obtain a much simpler relation among $Z_{q, m, N}(0)$ and $P_{q, m, N}(0)$ in the special case when $L_{Q} \mid N$.

Lemma 6.4.3. Let $D$ be the smallest positive divisor of $q-1$ such that $(q-1) / D$ is coprime to $Q$, and let $N \mid q^{m}-1$ such that $D Q \mid N$. Then $Z_{q, m, N}(0)$ is related to $P_{q, m, N}(0)$ by the equation

$$
Z_{q, m, N}(0)=\frac{q^{m}-1}{N} P_{q, m, N}(0)
$$

In particular we have the following relation:

$$
P_{q, m, q^{m}-1}(0)=\frac{\phi\left(q^{m}-1\right)}{\phi(D Q)} P_{q, m, D Q}(0) .
$$

Proof. First note that since $N$ is a multiple of $D Q$, then $\left(q^{m}-1\right) / N$ divides $(q-1) / D$ and hence is coprime to $Q$. Let $K$ be the largest divisor of $N$ that is coprime to $Q$. Then similarly as done in the proof of Lemma 6.2.1, we have, by Lemma 2.3.2,

$$
\begin{aligned}
\sum_{d \mid N} \mu(d) \sum_{i=0}^{q-2} \eta_{Q i}^{\left(\frac{q^{m}-1}{N} d, q^{m}\right)} & =\sum_{b \mid K} \sum_{d \mid \operatorname{gcd}(Q, N)} \mu(b d) \sum_{i=0}^{q-2} \eta_{Q i}^{\left(\frac{q^{m}-1}{N} b d, q^{m}\right)} \\
& =\sum_{b \mid K} \mu(b) \sum_{d \mid \operatorname{gcd}(Q, N)} \mu(d) \frac{(q-1) d}{\frac{q^{m}-1}{N} b d} \eta_{0}^{\left(d, q^{m}\right)} \\
& =\frac{N}{Q} \sum_{b \mid K} \frac{\mu(b)}{b} \Delta_{0}(\operatorname{gcd}(Q, N))=\frac{N \phi(K)}{Q K} \Delta_{0}(\operatorname{gcd}(Q, N)) .
\end{aligned}
$$

Then by Lemma 6.4 .1 and using the fact that $\frac{\phi(N)}{N}=\frac{\phi(K)}{K} \frac{\phi(\operatorname{gcd}(Q, N))}{\operatorname{gcd}(Q, N)}$, we get

$$
\begin{aligned}
P_{q, m, N}(0) & =\frac{N}{q Q}\left(Q \frac{\phi(N)}{N}+\frac{\phi(K)}{K} \Delta_{0}(\operatorname{gcd}(Q, N))\right) \\
& =\frac{N \phi(K)}{q Q K}\left(\frac{Q}{\operatorname{gcd}(Q, N)} \phi(\operatorname{gcd}(Q, N))+\Delta_{0}(\operatorname{gcd}(Q, N))\right) .
\end{aligned}
$$


Now the first identity follows from Theorem 1.0.18.

For the second, Lemma 6.2.2 and the first identity gives

$$
\frac{(q-1) / D}{\phi((q-1) / D)} P_{q, m, q^{m}-1}(0)=Z_{q, m, Q}(0)=Z_{q, m, D Q}(0)=\frac{q-1}{D} P_{q, m, D Q}(0)
$$

Hence we obtain

$$
P_{q, m, q^{m}-1}(0)=\phi\left(\frac{q-1}{D}\right) P_{q, m, D Q}(0) .
$$

Now the result follows by noticing that, since $D Q$ is coprime to $(q-1) / D$, then

$$
\phi\left(q^{m}-1\right)=\phi\left(D Q \frac{q-1}{D}\right)=\phi(D Q) \phi\left(\frac{q-1}{D}\right) .
$$

Note that $L_{Q}:=D Q$ is the largest divisor of $q^{m}-1$ with the same radical as that of $Q$. Moreover whenever $P_{q, m, q^{m}-1}(0) \neq 0$, the ratio, of the number of primitive elements with zero trace, to the number of elements of order $L_{Q}$ with trace zero, is the same as that of the number of primitive elements to the number of elements of order $L_{Q}$.

As a consequence of Theorem 1.0.17 and Lemma 6.4.3 we obtain the following existence result. For this we will need the fact that for any $N \mid\left(q^{m}-1\right)$ and any $d \mid N$, the set of $N$-free elements is a subset of the set of $d$-free elements in $\mathbb{F}_{q^{m}}$.

Theorem 6.4.4. Let $m>1$ and $N \mid q^{m}-1$ such that $L_{Q} \mid N$, where $L_{Q}$ is the largest divisor of $q^{m}-1$ with the same radical as that of $Q=\left(q^{m}-1\right) /(q-1)$. Then there exists an element $\xi \in \mathbb{F}_{q^{m}}^{*}$ of order $N$ satisfying $\operatorname{Tr}_{\mathbb{F}_{q^{m}} / \mathbb{F}_{q}}(\xi)=0$ if and only if $m \neq 2$ and $(q, m) \neq(4,3)$.

Proof. First by Lemma 6.2.2 there exists a primitive element with trace 0 if and only 
if there exists a $Q$-free element with trace 0. By Theorem 1.0.17 this happens if and only if $m \neq 2$ and $(q, m) \neq(4,3)$. Since a $\left(q^{m}-1\right)$-free (primitive) element is also $d$-free for any divisor $d$ of $q^{m}-1$, if follows that $Z_{q, m, N}(0)>0$ if $m \neq 2$ and $(q, m) \neq(4,3)$; by Lemma 6.4 .3 we also have $P_{q, m, N}(0)>0$. On the other hand, if $m=2$ or $(q, m)=(4,3)$, suppose on the contrary that $P_{q, m, N}(0)>0$. Then by Lemma 6.4.3 we have $Z_{q, m, N}(0)>0$. Since $Q \mid N$, then $Z_{q, m, Q}(0)>0$, contradicting the fact that $Z_{q, m, Q}(0)=0$ when $m=2$ or $(q, m)=(4,3)$.

We apply Lemma 6.4.3 together with Corollary 1.0.25 to obtain the following consequence.

Theorem 6.4.5. Let $p$ be a Mersenne prime and let $Q=\left(p^{4}-1\right) /(p-1)=(p+$ $1)\left(p^{2}+1\right)$. Then the number of non-zero elements in $\mathbb{F}_{p^{4}}^{*}$ with order $2 Q$ and absolute trace zero is $\phi(2 Q) /(p+1)=2 \phi\left(p^{2}+1\right)$.

Proof. In this case $Q=(p+1)\left(p^{2}+1\right)$ and so the only prime diving both $p-1$ and $Q$ is 2 . Since 2 has multiplicity 1 in the factorization of $p-1$, it follows that $D=2$, where $D$ is as defined in Lemma 6.4.3. Then by Lemma 6.4.3 and Corollary 1.0.25 we get

$$
\begin{aligned}
P_{q, m, 2 Q}(0) & =\frac{\phi(2 Q)\left(\phi\left(p^{4}-1\right)-\phi\left(\frac{p^{4}-1}{p+1}\right)\right)}{p \phi\left(p^{4}-1\right)} \\
& =\frac{\phi(2 Q)}{p}-\frac{\phi(2 Q) \phi\left(\frac{p^{4}-1}{p+1}\right)}{p \phi\left(p^{4}-1\right)} .
\end{aligned}
$$

By Euler's product formula for $\phi$ and using the fact that $2 \mid p-1$ while $p+1$ is a 
power of 2 , note that

$$
\begin{aligned}
(p+1) \phi\left((p-1)\left(p^{2}+1\right)\right) & =(p+1)(p-1)\left(p^{2}+1\right) \prod_{l \mid(p-1)\left(p^{2}+1\right)}\left(1-\frac{1}{l}\right) \\
& =(p+1)(p-1)\left(p^{2}+1\right) \prod_{l \mid(p+1)(p-1)\left(p^{2}+1\right)}\left(1-\frac{1}{l}\right) \\
& =\phi\left(p^{4}-1\right) .
\end{aligned}
$$

Now from the fact that $\left(p^{4}-1\right) /(p+1)=(p-1)\left(p^{2}+1\right)$, the above yields

$$
\begin{aligned}
P_{q, m, 2 Q}(0) & =\frac{\phi(2 Q)}{p}-\frac{\phi(2 Q)}{p(p+1)} \\
& =\frac{\phi(2 Q)}{p+1}
\end{aligned}
$$

Remains to note that, since $p$ is a Mersenne prime,

$$
\frac{\phi(2 Q)}{p+1}=2\left(p^{2}+1\right) \prod_{l \mid p^{2}+1}\left(1-\frac{1}{l}\right)=2 \phi\left(p^{2}+1\right)
$$




\section{Chapter 7}

\section{Conclusion}

In this thesis we studied several problems on polynomials over finite fields.

We started in Chapter 2 by reviewing some basic preliminary concepts which are required in subsequent chapters. Then we moved on to the other chapters covering compositional inverses of permutation polynomials, complete mappings, existence of irreducibles with prescribed coefficients, and ennumeration of primitive and $N$-free elements with prescribed trace.

In Chapter 3 we have presented the results in [70] where we extended the decomposition method, first used in [81], to find the compositional inverses, and preimages in certain cases, of several more general classes of permutation polynomials over arbitrary finite fields. As a result we showed how the inverses of these classes can be written in terms of the inverses, over subspaces, of two other polynomials, $\bar{f}, \varphi$, where $\varphi$ is linearized. In some of these cases one is able to obtain both such inverses, thus obtaining the full explicit result. We also showed that by solving a system of linear equations we can obtain a linearized polynomial inducing the inverse map over subspaces on which a prescribed linearized polynomial induces a bijection. In the special case that the Dickson matrix in such a linear equation is a circulant matrix, 
and the characteristic $p$ of $\mathbb{F}_{q^{n}}$ does not divide $n$, the system may be solved quickly by writing said equation as a convolution and then applying an FFT. In addition, we gave the compositional inverse of a class of permutation polynomials generalizing those for which their inverses were obtained in [23] and [81], respectively. We also obtained the explicit compositional inverse of a class of permutation polynomials generalizing that whose inverse was recently given in [79]. However, in many of the results we imposed the stronger condition that $\varphi$ bijects the subspace $S_{\psi}$, which is not a necessary condition for a given polynomial $f$ to induce a permutation of $\mathbb{F}_{q^{n}}$. It would thus seem to be of interest to "ease" this requirement, or possibly get rid of it altogether by using a certain bijection $\phi: \mathbb{F}_{q^{n}} \rightarrow \psi\left(\mathbb{F}_{q^{n}}\right) \oplus \operatorname{ker}(\psi)$, instead of our injective map $\phi_{\psi}: \mathbb{F}_{q^{n}} \rightarrow \psi\left(\mathbb{F}_{q^{n}}\right) \oplus S_{\psi}$. The reason for the preference of $\operatorname{ker}(\psi)$ over $S_{\psi}$ is that in most of the cases of the permutation $f$, a necessary condition for it to be a permutation is that $\varphi$ is injective on $\operatorname{ker}(\psi)$. We for now leave this as future work.

In Chapter 4 we showed our results in [71]. There we gave the compositional inverses of linearized binomials permuting the kernel of the trace map under the assumption that it does not permute the entire finite field in question. Recently, the compositional inverses of linearized permutation binomials were obtained in [81]. The significance of our result lies in its applications to obtain the compositional inverses of certain classes of permutation polynomials relying on the trace map, say the complete mapping of Theorem 4.2.3. We gave the compositional inverse of this mapping there. In addition we obtained an extension of a class of complete mappings recently given in [84] as well as obtained a recursive construction of complete mappings involving multi-trace functions.

In summary, with regards to compositional inverses, the method first used in [81] and extended in [70] seems quite applicable, and it is expected that it can similarly 
be applied to several more classes of permutation polynomials. The main drawback seems to be that the inverse results are written in terms of the inverses of $\bar{f}, \varphi$, over subspaces, which one may not always be able to easily determine explicitly. It would be interesting to find more classes of permutation polynomials with nice explicit expressions for their compositional inverses.

In Chapter 5 we presented the new proof, obtained in [72], of the Hansen-Mullen irreducibility conjecture. The proof partly relied on a (seemingly novel) sufficient condition for a given polynomial to have an irreducible factor of degree $n$, as well as some of the ideas of Dorsey-Hales [28]. We wonder whether some of the techniques employed here can be extended to tackle the problem of existence of irreducibles with several prescribed coefficients, but we for now leave this to the consideration of the interested reader.

Finally in Chapter 6 we presented the results first attained in [69]. In particular we gave a very simple formula for the number of primitive elements in quartic extensions of Mersenne prime fields, having absolute trace zero. In contrast to the case of the irreducible polynomials, no such formulas were known, up to now, in any specific non-trivial case of the primitives. In addition, using the known explicit expressions for Gaussian periods in the semi-primitive case as well as the results on uniformity, we obtained in Corollary 1.0.23 and 1.0.27 an explicit formula for the number of $\mathrm{N}$ free elements with prescribed trace. We also derived in the last section of Chapter 6 an expression, in terms of Gaussian periods, for the number of elements with order $N$ having a prescribed trace. Here we managed to derive an explicit formula, in Theorem 6.4.5, for the number of elements in $\mathbb{F}_{p^{4}}$ with absolute trace zero and high order $2(p+1)\left(p^{2}+1\right)$, where $p$ is a Mersenne prime.

Previously Cohen [16] proved that, except for a small number of trivial exceptions, there exists a primitive element with any prescribed trace. That is, he showed that 
for all $c \in \mathbb{F}_{q}^{*}, P_{q, q^{m}}\left(q^{m}-1, c\right)>0$, while, if $m \neq 2$ and $(q, m) \neq(4,3)$, then $P_{q, q^{m}}\left(q^{m}-1,0\right)>0$. One can see this as a classification of all the pairs $(q, m)$ such that there exists an element of order $q^{m}-1$ in $\mathbb{F}_{q^{m}}$ with any prescribed trace. One could also consider the following more general problem:

(1) Classify all the triples $(q, m, N)$ such that there exists an element with order $N \mid q^{m}-1$ in $\mathbb{F}_{q^{m}}^{*}$ having any prescribed trace.

Additionally, the reader might be interested in the following related problems:

(2) Find more classes of triples $(q, m, c)$ for which explicit formulas may be obtained for the number of primitive elements in $\mathbb{F}_{q^{m}}$ with trace $c \in \mathbb{F}_{q}$, say in the style of Corollary 1.0.18.

(3) Classify all triples $(q, m, N)$ such that the number of elements in $\mathbb{F}_{q^{m}}$, with order $N$ and trace $c \neq 0$, is uniform in the trace. One could also consider specifically the case when $N=q^{m}-1$.

(4) Classify all triples $(q, m, N)$ such that the number of $N$-free elements in $\mathbb{F}_{q^{m}}$, with trace $c \neq 0$, is uniform in the trace, as in Theorem 1.0.26.

(5) Find tight bounds for the number of $N$-free, respectively of order $N$, with a prescribed trace. In particular, in the case of $c=0$, find tight bounds for the sum $\Delta_{0}(n)$ in Chapter 6, where $n$ divides $Q=\left(q^{m}-1\right) /(q-1)$.

\subsection{Appendix}

The following table gives the numbers of primitive elements in the quartic extensions of Mersenne prime fields having absolute trace zero, for the first ten Mersenne primes. See Corollary 1.0.25 for an explicit formula. SAGE software was used for the computations. 
Table 7.1: The number of primitive elements of $\mathbb{F}_{p^{4}}$ with absolute zero trace for the first ten Mersenne primes $p$

\begin{tabular}{|c|c|}
\hline Mersenne prime, $p$ & $Z_{p, 4, p^{4}-1}(0)$ \\
\hline $2^{2}-1$ & 8 \\
\hline $2^{3}-1$ & 80 \\
\hline $2^{5}-1$ & 6,912 \\
\hline $2^{7}-1$ & 464,256 \\
\hline $2^{13}-1$ & $111,974,400,000$ \\
\hline $2^{17}-1$ & $519,390,596,431,872$ \\
\hline $2^{19}-1$ & $30,572,599,504,748,544$ \\
\hline $2^{31}-1$ & $1,968,482,608,781,191,263,129,600,000$ \\
\hline $2^{61}-1$ & $2,159,465,982,279,294,537,199,679,191$, \\
& $374,585,254,935,265,280,000,000,000$ \\
\hline $2^{89}-1$ & $51,505,739,520,752,637,174,787,391,794,396,705,748,179$, \\
& $291,647,742,969,497,437,393,928,825,245,616,046,080$ \\
\hline
\end{tabular}




\section{Bibliography}

[1] M. Aigner and G.M. Ziegler, Proofs from the book, 4th ed., Springer-Verlag, Berlin, 2010.

[2] A. Akbary, S. Alaric, Q. Wang, On some classes of permutation polynomials, International Journal of Number Theory, Vol 4, No. 1 (2008), 121-133.

[3] A. Akbary, D. Ghioca, Q. Wang, On constructing permutations of finite fields, Finite Fields Appl. 17 (2011), no. 1, 51-67.

[4] A. Akbary, D. Ghioca, Q. Wang, On permutation polynomials with prescribed shape, Finite Fields Appl. 15 (2009), no. 2, 195-206.

[5] A. Akbary and Q. Wang, On polynomials of the form $x^{r} f\left(x^{(q-1) / l}\right)$, Int. J. Math. Math. Sci. (2007), Article ID 23408, 7 pages.

[6] E. Bach, J. Shallit, Algorithmic number theory, Vol. 1. Efficient algorithms. Foundations of Computing Series. MIT Press, Cambridge, MA, 1996.

[7] A. Barvinok, Matrices with prescribed row and column sums, Linear Algebra Appl. 436 (2012), no. $4,820-844$.

[8] J. Bourgain, Prescribing the binary digits of primes, II, Israel J. Math. 206 (2015), no. 1, 165182.

[9] R.P. Brent, P. Zimmermann, The great trinomial hunt, Notices Amer. Math. Soc. 58 (2011), no. 2, 233-239.

[10] A. Canteaut and M. Videau, Symmetric boolean functions, IEEE Transactions on Information Theory, Institute of Electrical and Electronics Engineers 51 (2005), no. 8, 2791-2811.

[11] X. Cao, L. Hu and Z. Zha, Constructing permutation polynomials from piecewise permutations, Finite Fields Appl. 26 (2014), 162-174. 
[12] L. Carlitz, A theorem of Dickson on irreducible polynomials, Proc. Amer. Math. Soc. 3 (1952), 693-700.

[13] F.N. Castro and L.A. Medina, Linear recurrences and asymptotic behaviour of exponential sums of symmetric boolean functions, Electr. J. Comb. 18 (2011), no. 2, paper \#P9.

[14] A. Çeşmelioglu, W. Meidl and A. Pott, Generalized Maiorana-McFarland class and normality of p-ary bent functions, Finite Fields Appl. 24 (2013), 105-117.

[15] P. Charpin and G. Kyureghyan, When does $G(x)+\gamma \operatorname{Tr}(H(x))$ permute $\mathbb{F}_{p^{n}}$ ?, Finite Fields Appl. 15 (2009), no. 5, 615-632.

[16] S.D. Cohen, Primitive elements and polynomials with arbitrary trace, Discrete Math. 83 (1990), no. $1,1-7$.

[17] S.D. Cohen, Explicit theorems on generator polynomials, Finite Fields Appl. 11, no. 3 (2005), $337-357$.

[18] S.D. Cohen, Primitive polynomials over small fields, Finite fields and applications, 197-214, Lecture Notes in Comput. Sci., 2948, Springer, Berlin, 2004.

[19] S.D. Cohen, Primitive polynomials with a prescribed coefficient, Finite Fields Appl. 12 (2006), no. $3,425-491$.

[20] S.D. Cohen, M. Prešern, Primitive finite field elements with prescribed trace, Southeast Asian Bull. Math. 29 (2005), no. 2, 283-300.

[21] S.D. Cohen, M. Prešern, The Hansen-Mullen primitive conjecture: completion of proof, Number theory and polynomials, 89-120, London Math. Soc. Lecture Note Ser., 352, Cambridge Univ. Press, Cambridge, 2008.

[22] S.D. Cohen and M. Prešern, Primitive polynomials with prescribed second coefficient, Glasgow Math. J. 48 (2006), 281-307.

[23] R. S. Coulter, M. Henderson, The compositional inverse of a class of permutation polynomials over a finite field, Bull. Austral. Math. Soc. 65 (2002), 521-526.

[24] R. S. Coulter, M. Henderson, R. Mathews, A note on constructing permutation polynomials, Finite Fields Appl. 15 (2009), no. 5, 553-557. 
[25] C. Ding, Cyclic codes from some monomials and trinomials, SIAM J. Discrete Mathematics, to appear.

[26] C. Ding, J. Yang, Hamming weights in irreducible cyclic codes, Discrete Math. 313 (2013), no. $4,434-446$.

[27] C. Ding, J. Yuan, A family of skew Hadamard difference sets, J. Combin. Theory Ser. A 113 (2006), 1526-1535.

[28] T. Dorsey and A. W. Hales, Irreducible coefficient relations. Sequences and their applicationsSETA 2012, 117-125, Lecture Notes in Comput. Sci., 7280, Springer, Heidelberg, 2012.

[29] A.B. Evans, Orthomorphism graphs of groups, Lecture Notes in Mathematics, volume 1535, Springer-Verlag (1992).

[30] S.Q. Fan and W.B. Han, p-Adic formal series and primitive polynomials over finite fields Proc. Amer. Math. Soc. 132 (2004), 15-31.

[31] N. Fernando and X. Hou, A piecewise construction of permutation polynomial over finite fields, Finite Fields Appl. 18 (2012), 1184-1194.

[32] R.W. Fitzgerald and J.L. Yucas, Irreducible polynomials over GF(2) with three prescribed coefficients, Finite Fields Appl. 9 (2003), 286-299.

[33] T. Garefalakis, Irreducible polynomials with consecutive zero coefficients, Finite Fields Appl. 14 (2008), no. 1, 201-208.

[34] J. Ha, Irreducible polynomials with several prescribed coefficients, Finite Fields Appl. 40 (2016), $10-25$.

[35] K.H. Ham and G.L. Mullen, Distribution of irreducible polynomials of small degrees over finite fields, Math. Comp. 67 (1998), no. 221, 337-341.

[36] W.B. Han, On Cohen's problem, Chinacrypt '96, Academic Press (China) (1996) 231-235 (Chinese).

[37] T. Hansen and G.L. Mullen, Primitive polynomials over finite fields, Math. Comp. 59 (1992), 639-643.

[38] X. Hou, Two classes of permutation polynomials over finite fields, J. Combin. Theory Ser A 118 (2011), no. 2, 448-454. 
[39] X. Hou, A new approach to permutation polynomials over finite fields. Finite Fields Appl. 18 (2012), no. $3,492-521$.

[40] Ç.K. Koç, Open Problems in Mathematics and Computational Science, Springer International Publishing, 2014.

[41] B. Koma, D. Panario, Q. Wang, The number of irreducible polynomials of degree $n$ over $\mathbb{F}_{q}$ with given trace and constant terms, Discrete Mathematics, 310 (2010), 1282-1292.

[42] K. Kononen, M. Moisio, M. Rinta-aho, K. Väänänen, Irreducible polynomials with prescribed trace and restricted norm, JP J. Algebra Number Theory Appl. 11 (2009), 223-248.

[43] E. N. Kuz'min, On irreducible polynomials over a finite field (Russian) Sibirsk. Mat. Zh. 30 (1989), no. 6, 98-109; translation in Siberian Math. J.

[44] E. N. Kuz'min, A class of irreducible polynomials over a finite field, (Russian) Dokl. Akad. Nauk SSSR 313 (1990), no. 3, 552-555; translation in Soviet Math. Dokl. 42 (1991), no. 1, 45-48.

[45] G.M. Kyureghyan, Constructing permutations of finite fields via linear translators, J. Combin. Theory Ser. A 118 (2011), no. 3, 1052-1061.

[46] Y. Laigle-Chapuy, Permutation polynomials and applications to coding theory, Finite Fields Appl. 13 (2007), 58-70.

[47] Y. Laigle-Chapuy, A note on a class of quadratic permutation polynomials over $\mathbb{F}_{2^{n}}$, Applied Algebra, Algebraic Algorithms and Error-Correcting Codes, in: Lecture Notes in Comput. Sci., vol. 4851, Springer, (2007), 130-137.

[48] R. Lidl, H. Niederreiter, Finite fields, 2nd ed., Encyclopedia Math. Appl., vol. 20, Cambridge University Press, Cambridge, (1997).

[49] J.H. van Lint and R.M. Wilson, A course in combinatorics, 2nd ed., Cambridge University Press, Cambridge, 2001.

[50] J. E. Marcos, Specific permutation polynomials over finite fields, Finite Fields Appl. 17 (2011), no. $2,105-112$.

[51] P. Mihăilescu, Primary cyclotomic units and a proof of Catalan's conjecture, J. Reine Angew. Math. 572 (2004), 167-195.

[52] G.L. Mullen and D. Panario, Handbook of finite fields, CRC Press, 2013. 
[53] G.L. Mullen, Q. Wang, Permutation polynomials of one variable, in: Handbook of Finite Fields, Chapman and Hall/CRC, (2013), Section 8.1, 215-230.

[54] A. Muratović-Ribić, A note on the coefficients of inverse polynomials, Finite Fields Appl. 13 (2007), no. 4, 977-980.

[55] A. Muratović-Ribić and E. Pasalic, A note on complete polynomials over finite fields and their applications in cryptography, Finite Fields Appl. 25 (2014), 306-315.

[56] K. Nyberg, Perfect non-linear S-boxes, Proc. Advances in Cryptology, EUROCRYPT (1991), LNCS, vol. 547, Springer, Heidelberg, (1992), 378-386.

[57] D. Panario and G. Tzanakis, A generalization of the Hansen-Mullen conjecture on irreducible polynomials over finite fields, Finite Fields Appl. 18 (2) (2012) 303-315.

[58] P. Pollack, Irreducible polynomials with several prescribed coefficients, Finite Fields Appl. 22 (2013), 70-78.

[59] D-B. Ren, On the coefficients of primitive polynomials over finite fields, Sichuan Daxue Xuebao 38 (2001), 33-36.

[60] P. Ribenboim, The New Book of Prime Number Records, 3rd ed., Springer-Verlag, New York, 1995.

[61] R.L. Rivest, A. Shamir, L.M. Adelman, A method for obtaining digital signatures and public-key cryptosystems, ACM Commun. Comput. Algebra 21 (1978), 120-126.

[62] A. Sade, Groupoides automorphes par le groupe cyclique, Canad. J. Math. vol. 9, (1957), 321335.

[63] S. Samardjiska and D. Gligoroski, Quadratic permutation polynomials, complete mappings and mutually orthogonal Latin squares, preprint.

[64] J. Schwenk and K. Huber, Public key encryption and digital signatures based on permutation polynomials, Electron. Lett. 34 (1998) 759-760.

[65] I. E. Shparlinski, On primitive polynomials, Prob. Peredachi Inform. 23 (1988), 100-103 (Russian). 
[66] P. Stănică, S. Gangopadhyay, A. Chaturvedi, A. K. Gangopadhyay and S. Maitra, Investigations on bent and negabent functions via the nega-Hadamard transform, IEEE Trans. Inf. Theory 58 (6) (2012) 4064-4072.

[67] Z. Tu, X. Zeng, L. Hu, Several classes of complete permutation polynomials, Finite Fields Appl. 25 (2014), 182-193.

[68] A. Tuxanidy, Q. Wang, Composed products and factors of cyclotomic polynomials over finite fields, Des. Codes Cryptogr. 69 (2013), 203-231.

[69] A. Tuxanidy and Q. Wang, On the number of $N$-free elements with prescribed trace, J. Number Theory 160 (2016), 536-565.

[70] A. Tuxanidy, Q. Wang, On the inverses of some classes of permutations of finite fields, Finite Fields Appl. 28 (2014), 244-281.

[71] A. Tuxanidy and Q. Wang, Compositional inverses and complete mappings over finite fields, Discrete Applied Mathematics, v.217 no.2 (2017), p.318-329

[72] A. Tuxanidy and Q. Wang, A new proof of the Hansen-Mullen irreducibility conjecture, Canadian Journal of Mathematics, http://dx.doi.org/10.4153/CJM-2017-022-1, Canadian Mathematical Society 2018.

[73] A. Tuxanidy and Q. Wang, Characteristic digit-sum sequences, Cryptogr. Commun. (2018) 10:705-717

[74] G. Tzanakis, On the existence of irreducible polynomials with prescribed coefficients over finite fields, Master's thesis, Carleton University, 2010, http://www.math.carleton.ca/ gtzanaki/mscthesis.pdf.

[75] D. Wan, Generators and irreducible polynomials over finite fields, Math. Comp. 66 (219) (1997) $1195-1212$.

[76] Q. Wang, On inverse permutation polynomials, Finite Fields Appl. 15 (2009), 207-213.

[77] Q. Wang, On generalized Lucas sequences, Combinatorics and Graphs: the twentieth anniversary conference of IPM, May 15-21, (2009), Contemporary Mathematics 531 (2010), 127-141.

[78] Q. Wang, Cyclotomy and permutation polynomials of large indices, Finite Fields Appl. 22 (2013), $57-69$. 
[79] $\mathrm{B} . \mathrm{Wu}$, The compositional inverse of a class of linearized permutation polynomials over $\mathbb{F}_{2^{n}}, n$ odd, arXiv:1305.1411v2 [math.CO], preprint (2013).

[80] B. Wu and Z. Liu, Linearized polynomials over finite fields revisited, Finite Fields Appl. 22 (2013), 79-100.

[81] B. Wu and Z. Liu, The compositional inverse of a class of bilinear permutation polynomials over finite fields of characteristic 2, Finite Fields Appl. 24 (2013), 136-147.

[82] B. Wu, Linearized and linearized derived permutation polynomials over finite fields and their compositional inverses (in Chinese), Ph.D thesis, University of Chinese Academy of Sciences, 2013.

[83] B. Wu, The compositional inverses of linearized permutation binomials over finite fields, arXiv:1311.2154v1 [math.NT], preprint (2013).

[84] B. Wu and D. Lin, Complete permutation polynomials induced from complete permutations of subfields, arXiv:1312.5502v1 [math.NT], preprint (2013).

[85] B. Wu and D. Lin, On constructing complete permutation polynomials over finite fields of even characteristic, Discrete Applied Math. 184 (2015), 213-222.

[86] G. Wu, N. Li, T. Helleseth and Y. Zhang, More classes of complete permutation polynomials over $\mathbb{F}_{q}$, arXiv:1312.4716v2 [cs.IT], preprint (2013).

[87] A. Wyn-jones, Circulants, (2008). Available at: http://www.circulants.org/circ/circall.pdf

[88] P. Yuan and C. Ding, Permutation polynomials over finite fields from a powerful lemma, Finite Fields Appl. 17 (2011), no. 6, 560-574.

[89] P. Yuan and C. Ding, Further results on permutation polynomials over finite fields. Finite Fields Appl. 27 (2014), 88-103.

[90] J.L. Yucas, Irreducible polynomials over finite fields with prescribed trace/prescribed constant term, Finite Fields Appl. 12 (2006), no. 2, 211-221.

[91] Z. Zha and L. Hu, Two classes of permutation polynomials over finite fields, Finite Fields Appl. 18 (2012), no. 4, 781-790.

[92] Z. Zha, L. Hu, and X. Cao, Constructing permutations and complete permutations over finite fields via subfield-valued polynomials Finite Fields Appl. 31 (2015), 162-177. 
[93] M. Zieve, Classes of permutation polynomials based on cyclotomy and an additive analogue, in: Additive Number Theory, Springer, (2010), 355-361. 